\title{
Sterile neutrino oscillations in MINOS and hadron production in $\mathrm{pC}$ collisions
}

\author{
Gemma Maria Tinti \\ St. Cross College, Oxford
}

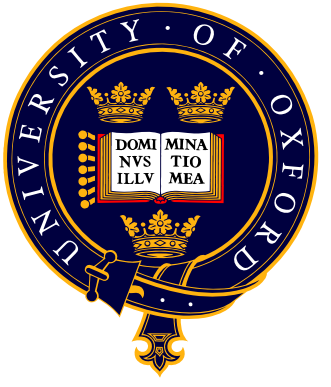

Thesis submitted in partial fulfilment of the requirements for the degree of Doctor of Philosophy at the University of Oxford

Trinity Term, 2010 


\title{
Sterile neutrino oscillations in MINOS and hadron production in $\mathrm{pC}$ collisions
}

\author{
Gemma Maria Tinti \\ St. Cross College, Oxford \\ Thesis submitted in partial fulfilment of the requirements for the degree of Doctor of \\ Philosophy at the University of Oxford
}

Trinity Term, 2010

\begin{abstract}
MINOS is a long baseline neutrino oscillation experiment, starting with a muon-neutrino beam, for the precise measurement of the atmospheric neutrino oscillation parameters $\left|\Delta m^{2}\right|$ and $\theta_{23}$. The Near Detector measures the neutrino flux and spectra before oscillations. The beam propagates for $735 \mathrm{~km}$ to the Far Detector, which measures the depleted spectrum after oscillations. The depletion can be interpreted as $\nu_{\mu} \rightarrow \nu_{\tau}$ oscillations. Subdominant $\nu_{\mu} \rightarrow \nu_{e}$ oscillations may be allowed if the mixing angle $\theta_{13} \neq 0$.

The two detectors are functionally identical in order to cancel systematic errors when using the Near Detector data to constrain the Far Detector prediction. A crucial part of the analysis is the relative calibration between the two detectors, which is known at the $2 \%$ level. A calibration procedure to remove the time and temperature dependence of the detector response using through-going cosmic muons is presented here.

Although the two-detector approach reduces the systematic uncertainties related to the neutrino flux, a cross check on the neutrino parent meson ratios is performed in this thesis. The cross sections of mesons produced in proton-carbon interactions from the NA49 experiment have been measured and the results have been compared to the MINOS expectations.

A neutrino oscillation analysis allowing mixing to a sterile neutrino is performed, under the assumption that the additional mass splitting is $\mathcal{O}\left(1 \mathrm{eV}^{2}\right)$. The analysis uses the energy spectrum of the neutral current interaction products, as neutral current interactions are sensitive to sterile neutrino mixing but not to the active flavour neutrino mixing. The neutrino oscillation parameters have been found to be: $\left|\Delta m^{2}\right|=2.43_{-0.18}^{+0.21} \times 10^{-3} \mathrm{eV}^{2}$, $\theta_{23}=40.27_{-5.17}^{\circ+14.64}, \theta_{24}=0.00^{\circ+5.99}$ and $\theta_{34}=0.00^{\circ+24.57}$ under the assumption that $\theta_{13}=12^{\circ}$ (limited by the CHOOZ experiment) or $\left|\Delta m^{2}\right|=2.43_{-0.18}^{+0.20} \times 10^{-3} \mathrm{eV}^{2}, \theta_{23}=39.09_{-4.89}^{\circ+16.71}$, $\theta_{24}=0.00^{\circ+4.79}$ and $\theta_{34}=0.00^{\circ+17.39}$ if $\theta_{13}$ is assumed to be zero.
\end{abstract}


To my parents 


\section{Contents}

1 Introduction $\quad 1$

2 Neutrinos $\quad 4$

2.1 First steps in the history of neutrinos . . . . . . . . . . . . . . 4

2.2 The neutrino interactions in the Standard Model and beyond . . . . . . . . . 6

2.2.1 (Neutrinoless) double $\beta$ decay . . . . . . . . . . . . . . . 7

2.2.2 Direct searches for neutrino mass . . . . . . . . . . . . . . 8

2.3 Neutrino oscillations ........................... 9

2.3.1 Neutrino oscillations in vacuum ................ 9

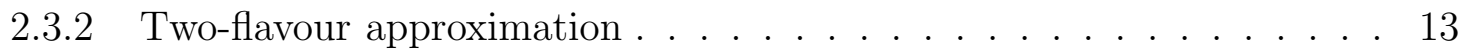

2.3.3 Neutrino oscillations in matter . . . . . . . . . . . . . . 17

2.3.4 Oscillation into sterile neutrinos . . . . . . . . . . . . . . 21

2.4 Experimental evidence . . . . . . . . . . . . . . . . . 25

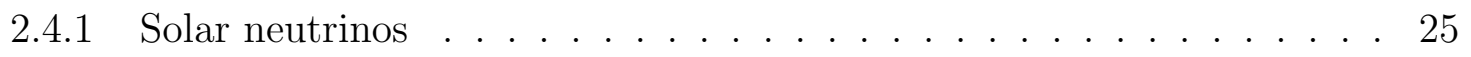

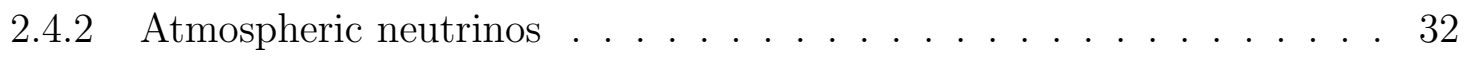

$2.4 .3 \quad \theta_{13}$ and $\delta \ldots \ldots \ldots \ldots \ldots \ldots \ldots$

2.4.4 Sterile neutrino hints . . . . . . . . . . . . . . . . 40

2.5 Conclusions: overview of global oscillation results . . . . . . . . . . . . 42

2.5.1 Solar $\Delta m^{2}$ mass splitting . . . . . . . . . . . . . . . 42

2.5.2 Atmospheric $\Delta m^{2}$ mass splitting . . . . . . . . . . . . . 44

2.5.3 Sterile $\Delta m^{2}$ mass splitting . . . . . . . . . . . . . . . . . . . 44 
3 The MINOS experiment $\quad 45$

3.1 The physics capabilities of MINOS . . . . . . . . . . . . . 45

3.2 The NuMI neutrino beam . . . . . . . . . . . . . . . . . 47

3.3 The MINOS detectors .................... . . 51

3.3.1 The Near Detector ................... 53

3.3.2 The Far Detector . . . . . . . . . . . . . . . . . 54

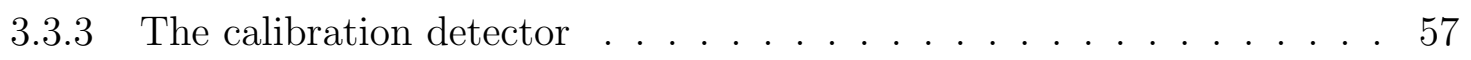

3.4 The MINOS Monte Carlo . . . . . . . . . . . . . . . . 57

3.5 Event Reconstruction . . . . . . . . . . . . . . . . . . . 59

3.6 Dataset analysed . . . . . . . . . . . . . . . . 60

4 The detector calibration in MINOS $\quad 61$

4.1 Detector calibration . . . . . . . . . . . . . . 61

4.2 The calibration chain . . . . . . . . . . . . . . . . 62

4.2.1 The data calibration chain . . . . . . . . . . . . . . . 62

4.2.2 The Monte Carlo calibration chain . . . . . . . . . . . . 66

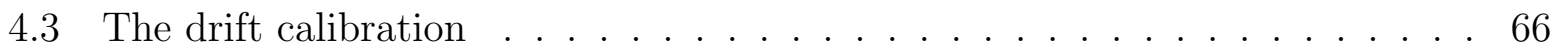

4.3.1 Cosmic muons . . . . . . . . . . . . . . . . . 68

4.3.2 The drift calibration technique . . . . . . . . . . . . . . 69

4.3.3 The drift calibration systematic errors . . . . . . . . . . . . 77

4.4 The photomultiplier gains and the scintillator light emission . . . . . . . . 79

4.5 Temperature dependences . . . . . . . . . . . . . . . 80

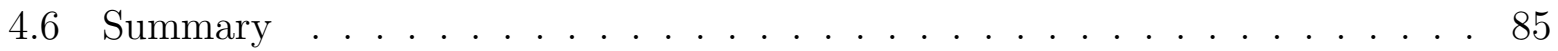

5 The NA49 Experiment $\quad 87$

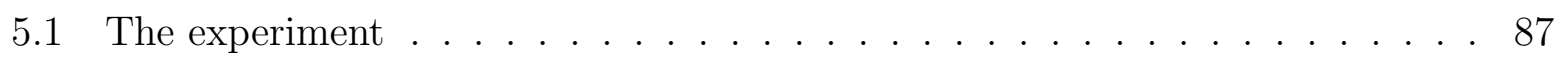

5.2 Beam detectors and interaction trigger . . . . . . . . . . . . . . 89

5.3 Targets . . . . . . . . . . . . . . . . . . . . 90

5.4 The Magnets . . . . . . . . . . . . . . . . . . . . . 90 
5.5 The Time Projection Chamber system . . . . . . . . . . . . . . . 90

5.6 Electronics and data acquisition . . . . . . . . . . . . . . . 93

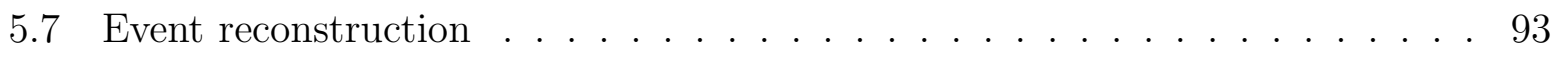

5.8 Data analysed . . . . . . . . . . . . . . . . 95

6 Inclusive invariant kaon production cross section in pC interactions 96

6.1 Energy loss by ionisation and particle identification . . . . . . . . . . . 97

$6.1 .1 d E / d x$ calibration steps $\ldots \ldots \ldots \ldots$

6.2 Event and track selection . . . . . . . . . . . . . . . 100

6.3 Binning in $x_{F}, p_{T} \ldots \ldots \ldots \ldots 1$

6.4 Detector response and modelling . . . . . . . . . . . . . . . . . 104

6.5 Particle identification using a fit to the specific ionisation . . . . . . . . 107

6.6 Errors on the fitted parameters . . . . . . . . . . . . . . . 110

6.7 Correlation of calibration parameters in $\mathrm{pC}$ and $\mathrm{pp}$ data . . . . . . . . 113

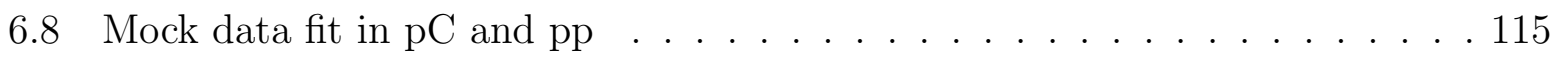

6.8.1 Particle separation in case of low statistics . . . . . . . . . 115

6.8.2 Cross check of the kaon yield errors . . . . . . . . . . . . 116

6.9 Double differential cross section . . . . . . . . . . . . . 116

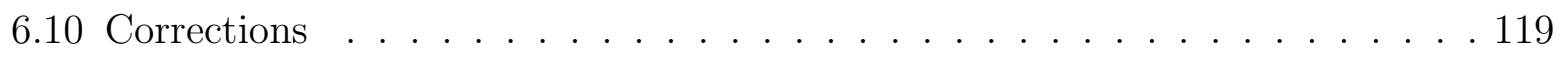

6.11 Systematic errors . . . . . . . . . . . . . . . . . . 120

6.12 Results . . . . . . . . . . . . . . . . . . . 122

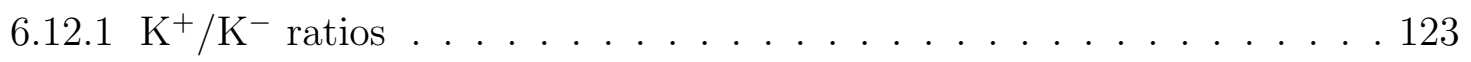

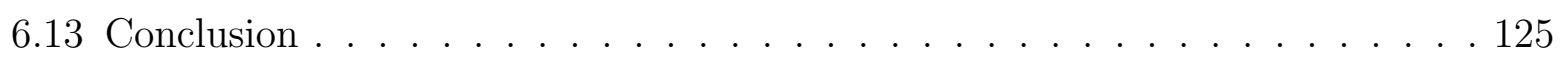

7 Neutrino flux in MINOS and comparison with the NA49 data 129

7.1 Results from $\mathrm{pC}$ interactions in NA49 . . . . . . . . . . . . . 130

7.1.1 Inclusive production cross section of pions . . . . . . . . . . 130

7.1.2 $\pi$ and $\mathrm{K} p_{T}$ integrated distributions . . . . . . . . . . . 131

7.1.3 Production of neutral kaons . . . . . . . . . . . . . . . 132 
7.2 Results from pC interactions from other experiments . . . . . . . . . . 132

7.3 Rate of neutrinos in MINOS . . . . . . . . . . . . . . . . 135

7.3.1 Tuning of the MINOS MC using Near Detector data . . . . . . . 138

7.4 Hadron production in MINOS and NA49 . . . . . . . . . . . . . 139

7.4.1 Comparison of particle ratios for different $p_{T}$ ranges . . . . . . . . 143

7.4.2 Comparison of particle ratios for different $p_{z}$ ranges . . . . . . . . . 146

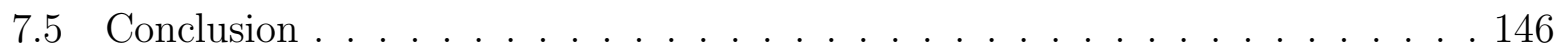

8 Sterile neutrino oscillations in MINOS 153

8.1 Analysis strategy . . . . . . . . . . . . . . . . . 153

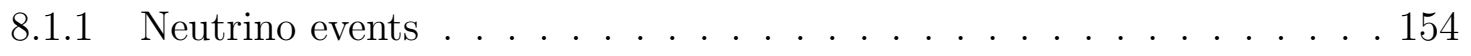

8.1.2 Sterile neutrino oscillations analysis . . . . . . . . . . . 155

8.2 Near Detector preselection . . . . . . . . . . . . . . . 158

8.2.1 Fiducial volume . . . . . . . . . . . . . . . . . . . 159

8.2.2 Definition of background . . . . . . . . . . . . . 160

8.2.3 Preselection Variables . . . . . . . . ......... 164

8.2.4 Systematic error evaluation ........................ 170

8.2.5 Discussion on the preselection ................ 174

8.3 Intensity effects at the Near Detector . . . . . . . . . . . . . 176

8.4 Far Detector preselection . . . . . . . . . . . . . . . 178

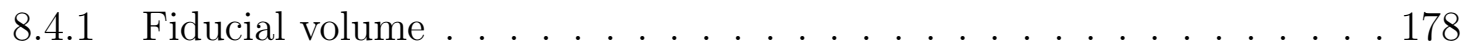

8.4 Light injection . . . . . . . . . . . . . . . 180

8.4.3 Wavelength-shifting fibre noise . . . . . . . . . . . . 181

8.4 .4 Cosmic muons . . . . . . . . . . . . . . . . 181

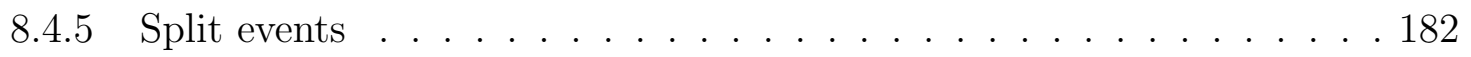

8.4.6 Timing requirements . . . . . . . . . . . . . . 182

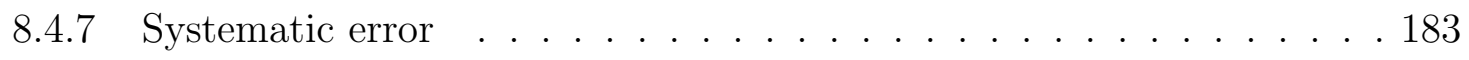

8.5 Selection of neutral current and charged current events . . . . . . . . . 183 
8.5.1 Neutral current selection . . . . . . . . . . . . . . . . . . . 184

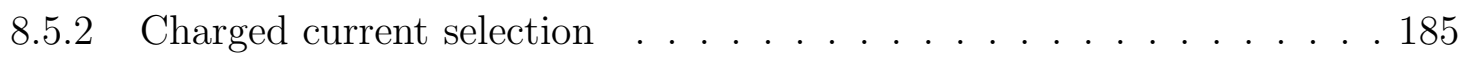

8.6 Far Detector prediction . . . . . . . . . . . . . . . 187

8.7 Systematic errors on the oscillation parameters . . . . . . . . . . . . 190

8.7.1 Sources of systematic errors common to the two detectors . . . . . . . 191

8.7.2 Sources of systematic errors causing differences between the two de-

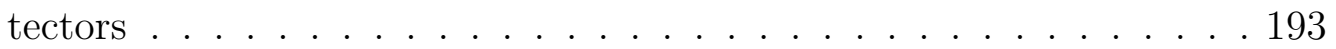

8.7.3 Effects of the systematic uncertainties on the oscillation parameters . 194

8.8 Sensitivity studies . . . . . . . . . . . . . . . . . 196

8.9 Results of the sterile neutrino oscillation analysis . . . . . . . . . . . 198

8.9.1 Data versus three-flavour oscillation prediction . . . . . . . . . 198

8.9.2 Data fitted to the $m_{4} \gg m_{3}$ model . . . . . . . . . . . . . 199

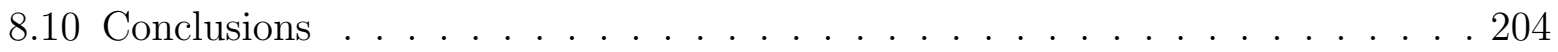

9 Conclusion $\quad 207$

$\begin{array}{ll}\text { Bibliography } & 209\end{array}$ 


\section{List of Figures}

1.1 Number of active neutrinos constraint by the $Z^{0}$ width . . . . . . . . . . 2

2.1 Possible neutrino mass hierarchies . . . . . . . . . . . . . . . . . 14

2.2 The survival probability in the two-flavour model as a function of neutrino

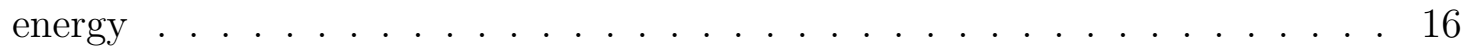

2.3 The survival probability for $\nu_{e}$ as a function of $L / E \ldots \ldots$. . . . . . . . 17

2.4 Coherent forward scattering diagrams of neutrinos on electrons . . . . . . . . 18

2.5 Possible neutrino mass hierarchies with a fourth mass state . . . . . . . . . 24

2.6 The experimental allowed ranges for the angles in the $3+1$ sterile neutrino

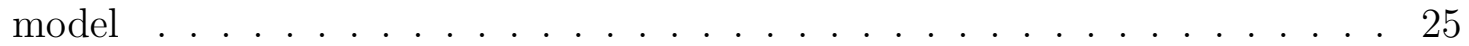

2.7 Predicted SSM solar neutrino fluxes and energy spectra . . . . . . . . . . 27

2.8 The flux of $\nu_{e}$ and $\nu_{\mu}+\nu_{\tau}$ from the Sun measured with the SNO detector . . 29

2.9 KamLAND oscillation results . . . . . . . . . . . . . . . . 30

2.10 Early atmospheric neutrino fluxes results . . . . . . . . . . . . . . 33

2.11 Zenith angle atmospheric neutrino distributions measured by the SuperKamiokande detector . . . . . . . . . . . . . . . . . . . 34

2.12 Super-Kamiokande ratio of data and unoscillated prediction as a function of

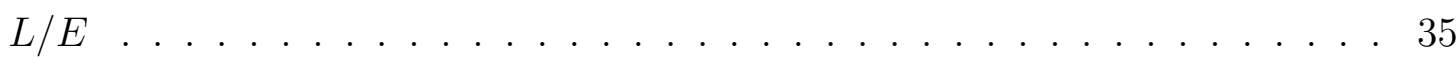

2.13 The MINOS oscillation parameters contour in comparison with other exper-

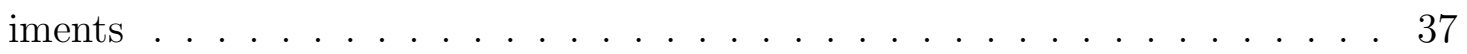

2.14 MINOS allowed region for $\theta_{13}$ in comparison with CHOOZ results . . . . . . 38 
2.15 Oscillation parameters excluded by the MiniBooNE experiment . . . . . . . 41

2.16 A summary of the constraints on neutrino oscillation parameters . . . . . . 43

3.1 The MINOS Far Detector charged current selected spectrum and ratio of the oscillated to the unoscillated spectrum . . . . . . . . . . . . . 46

3.2 Contours for the angle parameters in a sterile neutrino mixing model $\Delta m_{43}^{2} \approx$ $\mathcal{O}\left(1 \mathrm{eV}^{2}\right) \ldots \ldots \ldots \ldots \ldots \ldots$

3.3 The NuMI beamline . . . . . . . . . . . . . . . . . . . . 49

3.4 Three possible configurations of the NuMI beam . . . . . . . . . . . . 50

3.5 The MINOS near and far detectors . . . . . . . . . . . . . . . . . 52

3.6 A scintillator strip . . . . . . . . . . . . . . . . . 52

3.7 A cartoon of a MINOS detector alignment . . . . . . . . . . . . . . 52

3.8 The scintillator module arrangement at the Near Detector . . . . . . . . . . 55

3.9 The scintillator module arrangement on a Far Detector plane . . . . . . . . . 56

3.10 Energy resolution as function of the energy for different particles in the Calibration Detector . . . . . . . . . . . . . . . . . . . . . 58

3.11 Integrated number of POTs and neutral current events at the Far Detector . 60

4.1 The "track window" technique. . . . . . . . . . . . . . . . 65

4.2 A Far Detector cosmic muon. . . . . . . . . . . . . . . . . . 70

4.3 Number of selected cosmic muons per day. . . . . . . . . . . . . . . . 72

4.4 Pulse height per plane deposited by cosmic muon tracks in the detectors . . 74

4.5 The detectors drift. . . . . . . . . . . . . . . . . . . . 76

4.6 Spatial variation of the drift calibration constants. . . . . . . . . . . . . 77

4.7 Spatial variation of the drift calibrated pulse heights. . . . . . . . . . . 78

4.8 Drift calibration systematic errors. . . . . . . . . . . . . . . 79

4.9 Detector drift, photomultiplier gain and scintillation light percentage variation. 81

4.10 The Near Detector drift and temperature. . . . . . . . . . . . . . 83

4.11 The Far Detector drift and temperature. . . . . . . . . . . . . . . . . 84 
4.12 Detectors drift variation over temperature variation. . . . . . . . . . 85

5.1 The NA49 experiment layout. . . . . . . . . . . . . . . . 88

5.2 Schematic diagram of the TPCs readout system . . . . . . . . . . . . . 92

5.3 Example of an event display in NA49 . . . . . . . . . . . . . . . . . 94

6.1 The stopping power of muons in copper . . . . . . . . . . . . . . . . . 98

6.2 Energy deposited in the NA49 TPCs as a function of particle momentum . . 99

6.3 Binning scheme in $x_{F}$ and $p_{T} \ldots \ldots$. . . . . . . . . . . . . . 102

6.4 Number of charge clusters sampled for each track . . . . . . . . . . . 103

6.5 Azimuthal angle geometry . . . . . . . . . . . . . . . . . . 104

6.6 Distributions of the number of clusters sampled along tracks as a function of $\phi 105$

6.7 Resolution as a function of the specific ionisation and number of points sampled106

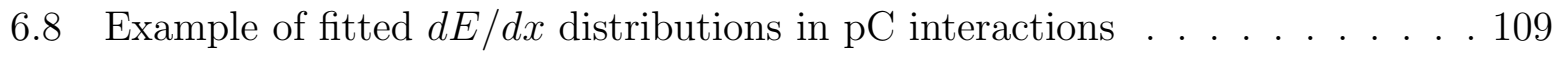

6.9 Projections of the $\Delta \chi^{2}$ curve in respect to the amplitude of kaons . . . . . 110

6.10 Kaons yield error divided by the Poisson error as a function of $x_{F}$. . . . 112

6.11 Separation parameter for the peaks in the fit . . . . . . . . . . . . 112

6.12 Measured percentage error on the number of kaons in $\mathrm{pC}$ interactions . . . . 113

6.13 Measured percentage error on the number of kaons in pp interactions . . . . 114

6.14 Correlation of the measured correction to the expected $d E / d x$ for the pions in $\mathrm{pC}$ and $\mathrm{pp} \ldots \ldots \ldots \ldots \ldots \ldots \ldots$

6.15 Fit results for mock data in $\mathrm{pC}$ and $\mathrm{pp} \ldots$. . . . . . . . . . . . . 117

6.16 Expected percentage error on the number of kaons in $\mathrm{pC}$ interactions . . . 118

6.17 Corrections to the kaon invariant cross sections . . . . . . . . . . . . . 121

6.18 Effect of the $d E / d x$ modelling systematic error . . . . . . . . . . . . . . . 122

$6.19 \mathrm{~K}^{+}$inclusive invariant cross section results . . . . . . . . . . . . 126

$6.20 \mathrm{~K}^{-}$inclusive invariant cross section results . . . . . . . . . . . . . . 127

$6.21 \mathrm{~K}^{+} / \mathrm{K}^{-}$production cross sections in NA49 . . . . . . . . . . . . . 128 
7.1 Pion inclusive invariant cross sections . . . . . . . . . . . . . . . . 130

$7.2 p_{T}$ integrated distribution for $\pi^{+} / \pi^{-}$and $\mathrm{K}^{+} / \mathrm{K}^{-} \ldots \ldots . \ldots 131$

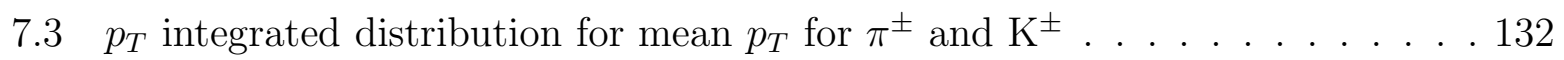

$7.4 p_{T}$ integrated cross sections as a function of $x_{F}$ for $\mathrm{K}^{ \pm}$and $\mathrm{K}^{0} \ldots \ldots 3$

$7.5\left(p_{z}, p_{T}\right)$ phase space covered by experimental results on kaon production in

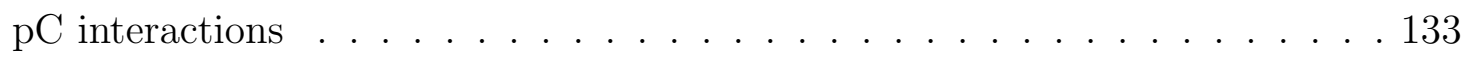

7.6 Kaon cross sections in Barton et al. as a function of $x_{F} \ldots \ldots 134$

$7.7\left(p_{z}, p_{T}\right)$ for $\pi^{+}$generating $\nu_{\mu}$ which interact in the Near Detector $\ldots 136$

$7.8\left(p_{z}, p_{T}\right)$ for $\mathrm{K}^{+}$generating $\nu_{\mu}$ which interact in the Near Detector $\ldots . . .137$

7.9 Neutrino events at the Near Detector decomposed according to the parent

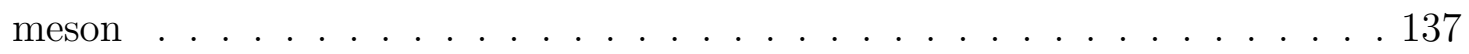

7.10 MC tuning of the charged current energy spectrum at the Near Detector . . 139

$7.11 \pi^{+} / \pi^{-}$as a function of $p_{z} \ldots \ldots \ldots 14 \ldots \ldots \ldots$

$7.12 \mathrm{~K}^{+} / \mathrm{K}^{-}$as a function of $p_{z} \ldots \ldots \ldots$. . . . . . . . . . . . . . . . . . . . . . . .

$7.13 \mathrm{~K}^{+} / \pi^{+}$and $\mathrm{K}^{-} / \pi^{-}$as a function of $p_{z} \ldots \ldots \ldots$. . . . . . . . . . 142

7.14 Comparison of $\pi^{+} / \pi^{-}$for different $p_{T} \ldots \ldots$. . . . . . . . . . . . . . 144

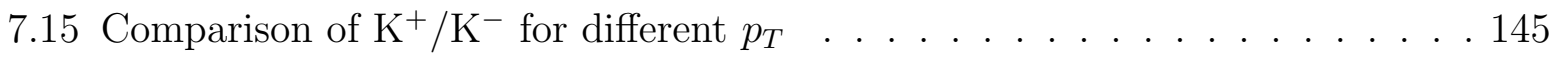

7.16 Comparison of $\mathrm{K}^{+} / \pi^{+}$for different $p_{T} \ldots \ldots \ldots$. . . . . . . . . 147

7.17 Comparison of $\mathrm{K}^{-} / \pi^{-}$for different $p_{T} \ldots \ldots$. . . . . . . . . . . . 148

7.18 Comparison of $\mathrm{K}^{+} / \mathrm{K}^{-}$for different $p_{z} \ldots \ldots \ldots$. . . . . . . . . . 149

7.19 Comparison of $\mathrm{K}^{+} / \pi^{+}$for different $p_{z} \ldots \ldots$. . . . . . . . . . . . 150

7.20 Comparison of $\mathrm{K}^{-} / \pi^{-}$for different $p_{z} \ldots \ldots \ldots$. . . . . . . . 151

8.1 Event display of $\nu_{\mu}$ charged current and neutral current MC events at the Far Detector . . . . . . . . . . . . . . . . . . 155

8.2 Event display of $\nu_{e}$ charged current and neutral current $\mathrm{MC}$ events at the Far Detector . . . . . . . . . . . . . . . . . 156

8.3 Fiducial volume cuts . . . . . . . . . . . . . . . . . 160 
8.4 Mean reconstructed visible energy as a function of the position in the detector 161

8.5 Example of a split event . . . . . . . . . . . . . . . . . . 162

8.6 Example of a leakage event . . . . . . . . . . . . . . . . 163

8.7 Reconstructed over true shower energy . . . . . . . . . . . . . . . 164

8.8 Event time distribution during a spill trigger . . . . . . . . . . . . . 165

8.9 Preselection variables . . . . . . . . . . . . . . . . 166

8.10 Neutral current selected energy spectrum before and after the preselection

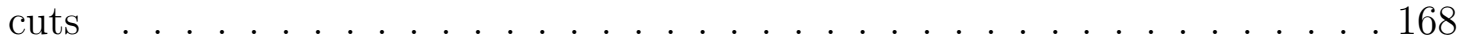

8.11 Preselection variables N-1 plots . . . . . . . . . . . . . . . 169

8.12 Efficiency and purity of selecting neutral current events . . . . . . . . . . 170

8.13 Month by month stability of the selected energy spectrum . . . . . . . . 171

8.14 Evaluation of the ND preselection systematic error . . . . . . . . . . 173

8.15 Systematic error band associated with the preselection cuts . . . . . . . . 174

8.16 Distribution of the difference between showers and tracks time at the Near

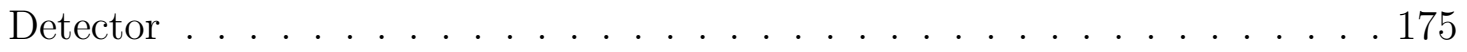

8.17 Event per spill trigger as a function of the beam intensity . . . . . . . . . 177

8.18 Beam intensity scaling on Run I . . . . . . . . . . . . . . . . 179

8.19 The distribution of the event vertices at the Far Detector after the fiducial volume cuts are applied . . . . . . . . . . . . . . . . . 180

8.20 Efficiency and purity of selected neutral current events at the Far Detector . 185

8.21 The neutral current selection variables at the Far Detector . . . . . . . 186

8.22 The neutral current selection variables at the Near Detector . . . . . . . . 186

8.23 Efficiency and purity of selecting charged current events . . . . . . . . . . . 187

8.24 Effect of the systematic errors on the Far Detector selected energy spectra after the full extrapolation is performed . . . . . . . . . . . . . 195

8.25 Shifts of the systematic errors on the oscillation parameters . . . . . . . 196

8.26 Sensitivities of the angle parameters . . . . . . . . . . . . . . . 197

8.27 Neutral current and charged current selected spectra at the Near Detector . 198 
8.28 Neutral current and charged current selected spectra at the Far Detector compared to the three-flavour prediction . . . . . . . . . . . . 200

8.29 Neutral current selected fitted spectrum at the Far Detector . . . . . . . . . 202

8.30 The $\Delta \chi^{2}$ projection for the $\Delta m^{2}$ parameter . . . . . . . . . . . . . . 202

8.31 The $\Delta \chi^{2}$ projections for the angle parameters . . . . . . . . . . . . . 203

$8.3290 \%$ C.L. contours for the angle parameters . . . . . . . . . . . . 203

8.33 The $\Delta \chi^{2}$ projection as a function of $\theta_{13} \ldots \ldots \ldots$. . . . . . . . . 206 


\section{List of Tables}

2.1 Direct measurements on neutrinos mass limits . . . . . . . . . . . . . 8

2.2 Solar reactions producing neutrinos . . . . . . . . . . . . . 26

4.1 Temperature dependence of detector drift, photomultiplier gains and scintillation light. . . . . . . . . . . . . . . . . . . . 82

5.1 NA49 target specifications. . . . . . . . . . . . . . . . . 90

5.2 Geometrical dimensions of the NA49 TPC system. . . . . . . . . . . . . 92

6.1 Sources of systematic errors in the kaon inclusive invariant cross section

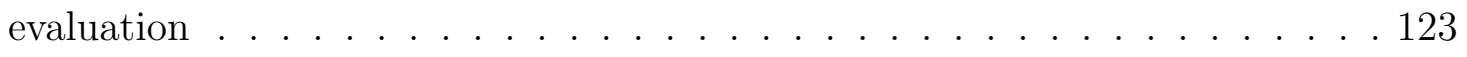

6.2 Invariant cross sections for $\mathrm{K}^{+} \ldots \ldots \ldots$. . . . . . . . . . . 124

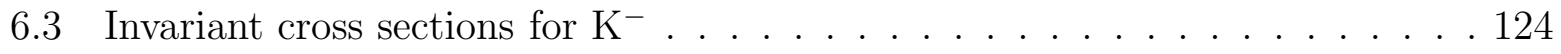

6.4 Ratio of the $K^{+}$to the $K^{-}$production cross section in NA49 . . . . . . . 124

8.1 FD preselection systematic error . . . . . . . . . . . . . . 183

8.2 Results of the parameters of the sterile neutrino mixing fit . . . . . . . . 201

8.3 Fit results for the nuisance parameters . . . . . . . . . . . . . 201 


\section{Chapter 1}

\section{Introduction}

Neutrino physics represents a very lively topic which has developed in the last eighty years. A first challenge in neutrino physics comes from the difficulty of detection, as they are neutral particles and interact only weakly. The number of active flavours of neutrinos is constrained to be three $\left(\nu_{e}, \nu_{\mu}, \nu_{\tau}\right)$ by the experimental measurement of the width of the $Z^{0}$ boson, as shown in figure 1.1 .

In the Standard Model, neutrinos are supposed to be massless. This has been contradicted by experimental evidence. Neutrino oscillations are possible as neutrinos are produced as flavour eigenstates but their propagation happens as mass eigenstates. The difference in mass causes a phase difference during the propagation: when the neutrino interacts as a flavour eigenstate there is a non-zero probability for it to have changed flavour. Neutrino oscillations are dependent on the ratio $L / E$, where $L$ represents the propagation length and $E$ is the neutrino energy: for the Main Injector Neutrino Oscillation Search (MINOS) experiment $L / E \approx 400 \mathrm{~km} / \mathrm{GeV}$.

This thesis studies neutrino oscillation parameters in the case of mixing to a fourth neutrino flavour ("sterile" as it cannot couple to the $Z^{0}$ ). In neutral current interactions, the only detectable product of the interaction is given by the hadronic shower. There is no possibility to distinguish among neutrino flavors and in the case of only the active flavour neutrino oscillations, the neutral current spectrum is expected to be unvaried. The signal for 


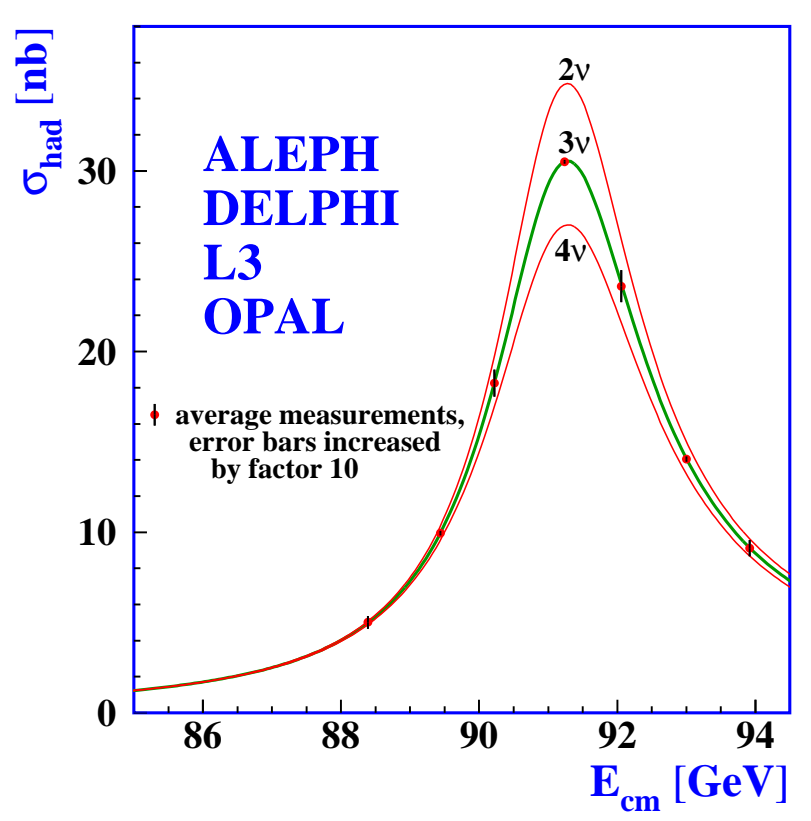

Figure 1.1: Number of active neutrinos as measured by the visible width of the $Z^{0}$ resonance from [1]. Only models with three active flavours are compatible with the data.

sterile neutrino oscillations would be a depletion of the neutral current spectrum. Chapter 2 introduces the theory behind neutrino oscillations and reviews the current experimental evidence.

Chapter 3 gives an overview of the MINOS physics potential and the experiment setup. MINOS is a long baseline neutrino beam experiment which employs two detectors (the Near Detector and the Far Detector) to measure the $\nu_{\mu}$ oscillation parameters.

Chapter 4 presents the MINOS detector calibration chain. The detector response is corrected for time, temperature and spatial variations and the pulse height is normalised to the same physical energy deposition in both the Near Detector and Far Detector data and Monte Carlo (MC). Work presented in this thesis contributes to the correction for time and temperature changes.

Using the Near Detector data to constrain the Far Detector prediction, the beam flux uncertainties are made non-critical in MINOS. Nevertheless, the comparison to direct measurements of hadrons off the target has been studied in this thesis. Chapter 5 introduces the NA49 experiment which has collected data from proton-carbon interactions with pri- 
mary proton momentum of $158 \mathrm{GeV} / c$ and a thin target. Chapter 6 describes the analysis performed and the results obtained. The interpretation of the results and their relevance for MINOS are presented in chapter 7. In the future, this work needs to be further expanded to provide a full interpretation of the hadron production uncertainties in MINOS.

The sterile neutrino oscillation analysis is presented in chapter 8 . The high rate of neutrinos causes reconstruction failures to be more frequent at the Near Detector. As a consequence, special emphasis is given to the selection at the Near Detector. The analysis procedure is presented and results are discussed. Final considerations are given in chapter 9. 


\section{Chapter 2}

\section{Neutrinos}

\subsection{First steps in the history of neutrinos}

The existence of the neutrino (although with the name "neutron") was first postulated by Pauli in 1930 in his famous letter addressed to the participants of the conference on radioactivity in Tübingen [2] to allow momentum conservation in certain nuclear decays. It was only later, in 1934, that Fermi postulated the theory of $\beta$ decay [3], with a massless neutral particle, the electron neutrino $\nu_{e}$, being emitted.

The first experimental detection of the neutrino was made in 1953 by Reines and Cowan [4]. They used reactor antineutrinos $\bar{\nu}_{e}$ in an inverse $\beta$ decay reaction $\bar{\nu}_{e}+p \rightarrow e^{+}+n$. The high neutrino flux $\left(\approx 10^{13} \mathrm{~cm}^{-2} \mathrm{~s}^{-1}\right)$ from the reactor compensated for the extremely small neutrino interaction cross section which was expected $\left(\approx 6 \times 10^{-44} \mathrm{~cm}^{2}\right)[5]$. The detector was made of liquid scintillator alternated with cadmium chloride and water layers. The signature of the products of this reaction is a prompt signal given by the annihilation of the positron in the scintillator and by a delayed signal from the captured neutron. The role of the cadmium target was to moderate the neutrons and enhance their absorption.

In 1958 Goldhaber, Grodzins and Sunyar measured the neutrino helicity [6] in a beautiful and sophisticated experiment studying the products from the K-capture of ${ }^{153} \mathrm{Eu}$. The neutrinos were measured to have only left-handed helicity. 
In 1962 at Brookhaven, an experimental group succeeded in directly detecting the second flavour of neutrino: the $\nu_{\mu}[7]$. A beam consisting of $\nu_{\mu}$, obtained from a proton beam colliding on a beryllium target, was produced from $\pi^{ \pm} \rightarrow \mu^{ \pm}+\nu(\bar{\nu})$. The pions were allowed to decay and then absorbing material was used to remove the hadrons and muons, leaving a beam of neutrinos. The Brookhaven experiment observed that only muons were produced by the interacting neutrinos. Lepton family number was shown to be conserved by neutrino interactions.

In 1973 the Gargamelle bubble chamber experiment at CERN released results on neutrino interactions. A beam of a mixture of $\nu_{e}, \nu_{\mu}$ and $\bar{\nu}_{\mu}$ was directed towards the bubble chamber. Neutrinos of lepton flavour $l$ were found $[8,9]$ to interact through charged current (CC) interactions:

$$
\nu_{l}+N \rightarrow l+X
$$

characterised by a charged lepton $l$ and a hadronic recoil $X$ in the final state. Neutrinos could also interact through neutral current (NC) processes:

$$
\nu_{l}+N \rightarrow \nu_{l}+X
$$

characterised by only the hadronic recoil $X$ in the final state. While charged current interactions, mediated by the charged $W$ boson, had already been observed, this was the first time that neutral current interactions (that had to be mediated by a neutral $Z^{0}$ boson) were detected. The collaboration also confirmed that strangeness-changing neutral current interactions such as:

$$
\begin{aligned}
& \nu N \rightarrow \nu \Lambda X \\
& \nu N \rightarrow \nu \Sigma^{0} X,
\end{aligned}
$$

where $X$ represents a non-strange hadron, were suppressed [10]. The experimental absence of flavour changing neutral currents, as well as the branching ratio for the decay of $\mathrm{K}^{0} \rightarrow$ 
$\mu^{+} \mu^{-}$, provided a starting point for Glashow, Iliopoulos and Maiani to postulate in 1970 [11] the existence of a new quark, "charm".

The existence of a third neutrino $\left(\nu_{\tau}\right)$ has now been theoretically and experimentally established. The indirect experimental evidence of the existence of the third neutrino was clear from the width of the $Z^{0}$ boson resonance peak at the Large Electron Positron collider at CERN. Assuming equal couplings to the $Z^{0}$ for all possible neutrinos, the fit to the width of the $Z^{0}$ allows $2.9840 \pm 0.0082$ neutrino flavours [12]. The measurement of the width of the $Z^{0}$ boson only constrains the number of neutrino flavours that interact weakly and have mass less than half the mass of the $Z^{0}$ boson. The direct observation of the $\nu_{\tau}$ was done much later, only in 2001, by the DONuT experiment [13]. The principle of the experiment is quite similar to the $\nu_{\mu}$ discovery experiments, but presents some more challenges due to the heaviness and short life-time of the $\tau$ lepton. A beam of $\nu_{\tau}$ was produced by colliding a beam of $800 \mathrm{GeV}$ protons on a thick tungsten target. The $D_{s}$ mesons leaving the target primarily decay into $\tau \nu_{\tau}$, from the same helicity arguments which make the pion prefer to decay to $\mu \nu_{\mu}$ rather than $e \nu_{e}$. The $\tau$ produced by the neutrino interaction (with a production threshold of $\approx 3.5 \mathrm{GeV}$ ) immediately decay into muons, which can be tracked by the alternating steel and emulsions. The emulsions are necessary to give sufficient spatial resolution to measure the "kink" from the $\tau$ decay into the muon $\left(c \tau_{\tau}=87.11 \mu \mathrm{m}[12]\right)$.

\subsection{The neutrino interactions in the Standard Model and beyond}

Neutrinos are massless fermions in the Standard Model (SM) of particle physics. The elec-

trically charged fermions in the SM are Dirac particles. The mass terms in the Lagrangian $\mathcal{L}_{\mathcal{D}}$ for charged fermions can be written as:

$$
\mathcal{L}_{\mathcal{D}}=-m_{D}\left(\bar{\psi}_{R} \psi_{L}+\bar{\psi}_{L} \psi_{R}\right)
$$


where $m_{D}$ is the Dirac mass coupling to the Higgs field. The two fields $\psi_{L}$ and $\psi_{R}$ are the chirality states of the fermion spinors. As neutrinos are neutral, there is still an ambiguity whether they are Dirac or Majorana particles. If neutrinos are massless, the neutrino is described only by the $\psi_{L}\left(=\nu_{L}\right)$ field and mass terms in the Lagrangian are prohibited.

To accommodate massive neutrinos, the SM needs to be modified. The $\psi_{R}\left(=\nu_{R}\right)$ field needs to be introduced for massive neutrinos. If $M_{M}$ is the Majorana mass for the neutrino, a term like $M_{M} \bar{\nu}_{R} \nu_{R}$ is allowed in the Lagrangian. Many different models exist, but they require the so-called "seesaw" mechanism to explain why neutrino masses are so small compared to the other fermion masses. The mass term in the Lagrangian is given by:

$$
\mathcal{L}_{\mathcal{D}+\mathcal{M}}=-\frac{1}{2}\left(\begin{array}{ll}
\bar{\nu}_{L} & \bar{\nu}_{R}
\end{array}\right)\left(\begin{array}{cc}
0 & m_{D} \\
m_{D} & M_{M}
\end{array}\right)\left(\begin{array}{c}
\nu_{L} \\
\nu_{R}
\end{array}\right)
$$

In the case $m_{D} \ll M_{M}$, from the diagonalization of the matrix, two eigenvalues are found. The heavy eigenvalue has mass $\approx M_{M}$, being the mass of $\nu_{R}$, while the light eigenstate gives the mass of the standard $\nu_{L}$ neutrino:

$$
m_{\nu} \approx \frac{m_{D}^{2}}{M_{M}}
$$

If the Majorana mass is of order of $10^{15} \mathrm{GeV}$, then the neutrino mass is naturally very small.

\subsection{1 (Neutrinoless) double $\beta$ decay}

If the neutrino is a Dirac particle, $\nu_{e}$ and $\bar{\nu}_{e}$ are different and processes like:

$$
2 n \rightarrow 2 p+2 e^{-}+2 \bar{\nu}_{e}
$$


can occur. On the other hand, if $\nu_{e}$ and $\bar{\nu}_{e}$ are the same particle, then neutrinoless double $\beta$ decay could also be possible:

$$
2 n \rightarrow 2 p+2 e^{-}
$$

The reaction can be thought of in two steps: the nucleus undergoes a first $\beta$ decay $n \rightarrow p+e^{-}+\bar{\nu}_{e}$. If the emitted $\bar{\nu}_{e}$ is equivalent to a $\nu_{e}$, the second transition $\nu_{e}+n \rightarrow p+e^{-}$can occur.

If neutrinoless double $\beta$ decay were to be observed experimentally, it would imply that lepton number is not conserved. Furthermore, it would be a proof of existence of Majorana neutrinos and the measurement can be sensitive to the neutrino mass ordering (referred to as the neutrino mass hierarchy). Many experiments are currently trying to search for neutrinoless double $\beta$ decay. To list the majors: CUORE [14], EXO [15] and Super-Nemo [16].

\subsubsection{Direct searches for neutrino mass}

The end-point of the energy spectrum of the electron in $\beta$ decays provides a method to be directly sensitive to the neutrino masses. If neutrinos were massless, the observed Kurie plots of the electron spectra would be linear. A deviation from linearity is expected in the case of a massive neutrino: to conserve energy and allow a massive neutrino, the electron end-point spectra should be slightly decreased. Experiments are currently trying to determine the neutrino mass directly from Kurie plot fits, although the instrumental limits are still quite large. The current best limits from a global fit [12] are summarised in table 2.1 .

\begin{tabular}{|l|c|}
\hline$m_{\nu_{e}}<2 \mathrm{eV}$ & from tritium decay \\
\hline$m_{\nu_{\mu}}<0.19 \mathrm{MeV}$ & from $\pi$ decay \\
\hline$m_{\nu_{\tau}}<18.2 \mathrm{MeV}$ & from $\tau$ decay \\
\hline
\end{tabular}

Table 2.1: The table shows the current average mass limits for the three neutrino flavours. Data from [12].

The Supernova SN 1987A has provided a unique source of low energy neutrinos with 
very long baseline. The flux of neutrinos detected on the Earth (from the Kamiokande, IMB and Baksan detectors) had a duration of nearly $10 \mathrm{~s}$, big enough to suppose that lighter neutrinos were arriving earlier than massive neutrinos. The limit on $m_{\nu_{e}}$ from the supernova measurement is compatible with the tritium limit [17].

\subsection{Neutrino oscillations}

Experimental results from the detection of neutrinos from the Sun and the atmosphere have required neutrino oscillations as a possible explanation for the missing neutrinos. Neutrino oscillations work with a mechanism similar to neutral kaon oscillations. To satisfy oscillations, neutrinos are required to have mass. Neutrino physics is the first evidence of phenomenology beyond the Standard Model (SM) of particle physics. Nevertheless, the theory can easily be accommodated as an "ad hoc" extension of the SM.

\subsubsection{Neutrino oscillations in vacuum}

Neutrinos interact weakly as flavour eigenstates: $\left|\nu_{e}\right\rangle,\left|\nu_{\mu}\right\rangle$ and $\left|\nu_{\tau}\right\rangle$. Their propagation depends on their mass eigenstates $\left|\nu_{1}\right\rangle,\left|\nu_{2}\right\rangle$ and $\left|\nu_{3}\right\rangle$. It is possible to relate the flavour eigenstates to the mass eigenstates through a rotation:

$$
\left|\nu_{\alpha}\right\rangle=\sum_{j} U_{\alpha j}^{*}\left|\nu_{j}\right\rangle
$$

where $\nu_{\alpha}=\left(\nu_{e}, \nu_{\mu}, \nu_{\tau}\right)$ represent the flavour states, $\nu_{j}=\left(\nu_{1}, \nu_{2}, \nu_{3}\right)$ represent the mass eigenstates with mass $m_{j} . U_{\alpha j}$ is called the PMNS rotation matrix, satisfying the unitarity condition $U^{\dagger} U=\mathbb{1}$. The PMNS rotation matrix is named after Pontecorvo, Maki, Nakagawa and Sakata, who formalised a two flavour neutrino oscillation theory in $[18,19]$. A neutrino is produced as a flavour eigenstate at a time $t=0\left|\nu_{\alpha}(t=0)\right\rangle$ but propagates 
depending on its mass eigenstates:

$$
\left|\nu_{\alpha}(t)\right\rangle=\sum_{j} U_{\alpha j}^{*} e^{\mathrm{i} p_{j} \cdot x}\left|\nu_{j}\right\rangle
$$

where $x$ is the four-position of the neutrino and $p_{j}$ the four-momentum of the mass state $j$. At a time $t$ the neutrino weakly interacts in a detector. Its wave function is therefore collapsed into a weak eigenstate $\left\langle\nu_{\beta}\right|=\sum_{i} U_{\beta i}\left\langle\nu_{i}\right|$ (a sum over mass eigenstates $i$ ) with amplitude:

$$
\begin{aligned}
\left\langle\nu_{\beta} \mid \nu_{\alpha}(t)\right\rangle & =\sum_{i} \sum_{j} U_{\beta i} U_{\alpha j}^{*} e^{\mathrm{i} p_{j} \cdot x}\left\langle\nu_{i} \mid \nu_{j}\right\rangle \\
& =\sum_{j} U_{\beta j} U_{\alpha j}^{*} e^{\mathrm{i} p_{j} \cdot x} .
\end{aligned}
$$

Assuming all the three mass eigenstates have the same three-momentum $\mathbf{p}$, but different energy $E_{j}$ according to the different mass $m_{j}, p_{j} \cdot x$ can be written as:

$$
\begin{aligned}
p_{j} \cdot x & =E_{j} t-\mathbf{p} \cdot \mathbf{x} \\
& =t \sqrt{|\mathbf{p}|^{2}+m_{j}^{2}}-\mathbf{p} \cdot \mathbf{x} .
\end{aligned}
$$

In natural units, $t$ can be expressed as $t=L$, where $L$ denotes the distance travelled at the speed of light $c$. Substituting $\mathbf{p} \cdot \mathbf{x}=|\mathbf{p}| L$ and performing a second order Taylor's expansion as $m_{j} \ll E_{j}$, equation (2.13) can be expressed as:

$$
\begin{aligned}
p_{j} \cdot x & =|\mathbf{p}| L\left(1+\frac{m_{j}^{2}}{2|\mathbf{p}|^{2}}\right)-|\mathbf{p}| L \\
& =\frac{m_{j} L}{2 E}
\end{aligned}
$$

where in the last step $|\mathbf{p}| \approx E$ for $m_{j} \ll E_{j}$. Thus, the probability of observing a neutrino 
of flavour $\nu_{\beta}$ a distance $L$ from a source of originally pure $\nu_{\alpha}$ is then:

$$
\begin{aligned}
P\left(\nu_{\alpha} \rightarrow \nu_{\beta}\right) & =\left|\left\langle\nu_{\beta} \mid \nu_{\alpha}(L)\right\rangle\right|^{2} \\
& =\left(\sum_{i} U_{\beta i}^{*} U_{\alpha i} e^{-\mathrm{i} \frac{m_{i}^{2} L}{2 E}}\right)\left(\sum_{j} U_{\beta j} U_{\alpha j}^{*} e^{\mathrm{i} \frac{m_{j}^{2} L}{2 E}}\right) \\
& =\sum_{j} \sum_{i} U_{\beta i}^{*} U_{\beta j} U_{\alpha j}^{*} U_{\alpha i} e^{-\mathrm{i} \frac{\Delta m_{j i}^{2} L}{2 E}} \\
& =\sum_{j} U_{\beta j} U_{\alpha j}^{*} \sum_{i} U_{\beta i}^{*} U_{\alpha i} \\
& +\sum_{j} \sum_{i} U_{\beta j} U_{\alpha j}^{*} U_{\beta i}^{*} U_{\alpha i}\left(e^{-\mathrm{i} \frac{\Delta m_{j i}^{2} L}{2 E}}-1\right) .
\end{aligned}
$$

Where $\Delta m_{j i}^{2}$ has been defined as $\Delta m_{j i}^{2}=m_{j}^{2}-m_{i}^{2}$. Splitting the complex exponential term into its real and imaginary parts and using some trigonometric adjustments, equation (2.15) can be rewritten as:

$$
\begin{aligned}
P\left(\nu_{\alpha} \rightarrow \nu_{\beta}\right) & =\delta_{\alpha \beta}-4 \sum_{j>i} \mathfrak{R e}\left(U_{\alpha j}^{*} U_{\beta j} U_{\alpha i} U_{\beta i}^{*}\right) \sin ^{2}\left(\frac{\Delta m_{j i}^{2} L}{4 E}\right) \\
& +2 \sum_{j>i} \mathfrak{I m}\left(U_{\alpha j}^{*} U_{\beta j} U_{\alpha i} U_{\beta i}^{*}\right) \sin \left(\frac{\Delta m_{j i}^{2} L}{2 E}\right)
\end{aligned}
$$

A further simplification in the notation can be applied defining $\Delta_{j i}$ as $\Delta_{j i} \equiv\left(m_{j}^{2}-m_{i}^{2}\right) L /(4 E)$.

The PMNS rotation matrix can be rewritten, in the standard parameterisation in terms 
of three mixing angles $\theta_{12}, \theta_{23}$ and $\theta_{13}$ and a phase $\delta$ as:

$$
\begin{aligned}
U= & {\left[\begin{array}{lll}
U_{e 1} & U_{e 2} & U_{e 3} \\
U_{\mu 1} & U_{\mu 2} & U_{\mu 3} \\
U_{\tau 1} & U_{\tau 2} & U_{\tau 3}
\end{array}\right] } \\
= & {\left[\begin{array}{ccc}
c_{12} c_{13} & s_{12} c_{13} & s_{13} e^{-\mathrm{i} \delta} \\
-s_{12} c_{23}-c_{12} s_{23} s_{13} e^{\mathrm{i} \delta} & c_{12} c_{23}-s_{12} s_{23} s_{13} e^{\mathrm{i} \delta} & s_{23} c_{13} \\
s_{12} s_{23}-c_{12} c_{23} s_{13} e^{\mathrm{i} \delta} & -c_{12} s_{23}-s_{12} c_{23} s_{13} e^{\mathrm{i} \delta} & c_{23} c_{13}
\end{array}\right]\left[\begin{array}{ccc}
1 & 0 & 0 \\
0 & e^{\mathrm{i} \alpha} & 0 \\
0 & 0 & e^{\mathrm{i} \beta}
\end{array}\right] } \\
= & {\left[\begin{array}{ccc}
1 & 0 & 0 \\
0 & c_{23} & s_{23} \\
c_{13} & 0 & s_{13} e^{-\mathrm{i} \delta} \\
0 & -s_{23} & c_{23}
\end{array}\right]\left[\begin{array}{ccc}
0 & 1 & 0 \\
c_{12} & 0 \\
-s_{13} e^{\mathrm{i} \delta} & 0 & c_{13}
\end{array}\right]\left[\begin{array}{ccc}
1 & c_{12} & 0 \\
0 & 0 & 1
\end{array}\right]\left[\begin{array}{ccc}
0 & e^{\mathrm{i} \alpha} & 0 \\
0 & 0 & e^{\mathrm{i} \beta}
\end{array}\right] }
\end{aligned}
$$

where $s_{i j}=\sin \theta_{i j}$ and $c_{i j}=\cos \theta_{i j}$. The unitarity constraint on $U$ removes nine free parameters, and five of the remaining are relative phases between the six lepton fields which can be absorbed by those fields. This leaves four free parameters in $U$. A Dirac phase $\delta$ allows for the possibility of $\mathrm{CP}$ violation in the lepton sector. The two Majorana phases $\alpha$ and $\beta$ are unobservable in neutrino oscillation experiments. Consequently, these phases are ignored here, but Majorana phases play an important role in neutrinoless double $\beta$ decay search experiments. The last equality does not apply any conceptual modification, but the mixing matrix is decomposed into terms that can be associated with different classes of experiments. The leftmost of the matrices, involving $\theta_{23}$ is studied by those experiments concerned with the atmospheric $\Delta m_{\mathrm{atm}}^{2}$. The second-to-rightmost matrix involving $\theta_{12}$ is studied by those experiments interested in the solar $\Delta m_{\odot}^{2}$. Finally, the matrix involving $\theta_{13}$ and $\delta$ is the current most interesting topic in the neutrino sector and will be intensively studied by future experiments.

Under the assumption of CPT being conserved, the first of the two equalities in equa- 
tion (2.18) is true:

$$
P\left(\bar{\nu}_{\alpha} \rightarrow \bar{\nu}_{\beta}\right) \stackrel{\mathrm{CPT}}{=} P\left(\nu_{\beta} \rightarrow \nu_{\alpha}\right) \stackrel{\mathrm{CP}}{=} P\left(\nu_{\alpha} \rightarrow \nu_{\beta}\right)
$$

The second equality holds if $U$ is not complex $(\delta=0)$, i.e. if CP is conserved.

Disappearance experiments, that is, experiments looking at the same neutrino flavour at production and detection, are not sensitive to $\mathrm{CP}$ violation, as the phase $\delta$ disappears in the formula for $P\left(\nu_{\alpha} \rightarrow \nu_{\alpha}\right)$. $\delta$ can be determined only by appearance experiments if all three mixing angles are non-zero ${ }^{1}$ : currently only an upper-bound limit has been put on $\theta_{13}$ and it could be zero. The matrix in equation (2.17) shows a convenient parametrisation as $\delta$ has been coupled with the term $s_{13}$ and does not affect measurements involving only $\theta_{12}$ and $\theta_{23}$.

The current best values of the parameters and the Confidence Level (C.L.) are [20]:

- $\theta_{12}=34.4^{\circ} \pm 1.0^{\circ}$ at $68 \%$ C.L.

- $\theta_{23}=42.3_{-2.8}^{\circ}+5.3$ at $68 \%$ C.L.

- $\theta_{13}=6.8^{\circ}{ }_{-3.6}^{+2.6}$ at $68 \%$ C.L.

- $0 \leq \delta \leq 2 \pi$

- $\Delta m_{21}^{2}=7.59 \pm 0.20 \times 10^{-5} \mathrm{eV}^{2}$ at $68 \%$ C.L.

- $\left|\Delta m_{32}^{2}\right|=2.40_{-0.11}^{+0.23} \times 10^{-3} \mathrm{eV}^{2}$ at $68 \%$ C.L.

\subsubsection{Two-flavour approximation}

Looking at the formulation of the $U$ matrix in equation (2.17) and considering the large difference in the two mass splittings $\Delta m_{21}^{2}$ and $\left|\Delta m_{32}^{2}\right|$ and the small value of $\theta_{13}$, it is easy to see that the neutrino oscillation problem can be decoupled into two different mass splitting sectors. Most experiments are sensitive only to one of the two mass splittings.

\footnotetext{
${ }^{1}$ As an example see equations 5 and 6 on page 540 in [12].
} 


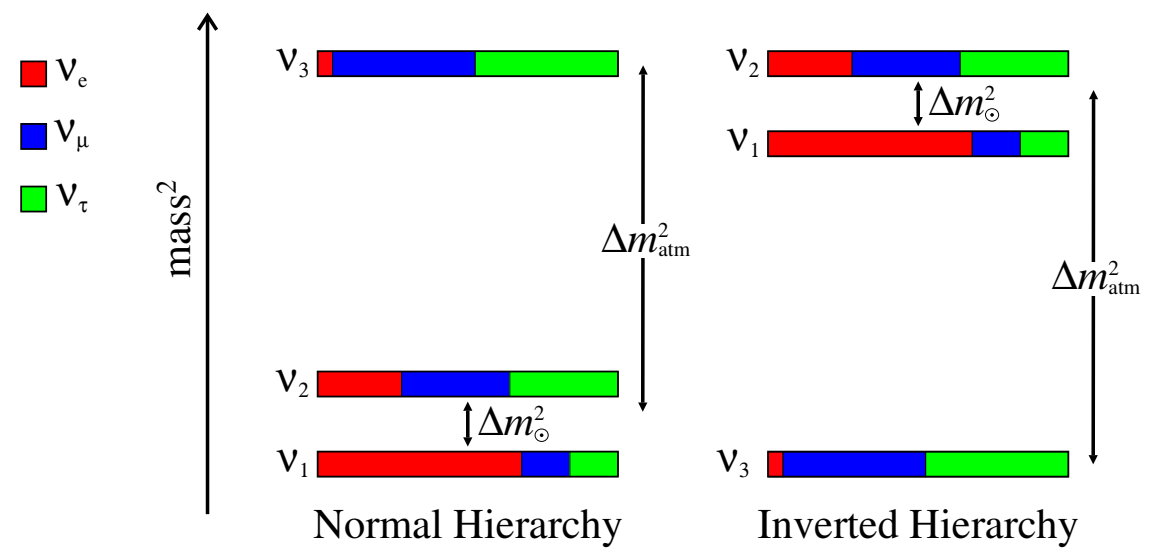

Figure 2.1: Schematic of the two allowed mass orderings given fixed $\Delta m_{\mathrm{atm}}^{2}$ and $\Delta m_{\odot}^{2}$. The diagram on the left is for the normal mass ordering, while the diagram on the right is for the inverted mass ordering. The colours show the approximate flavour content of each mass state. The figure is taken from [21].

Figure 2.1 shows the two possible mass orderings for the three-neutrino case that allow $\Delta m_{\text {atm }}^{2} \gg \Delta m_{\odot}^{2}$. They are usually referred to as the "normal" and the "inverted" mass hierarchy. Note that the mass ordering for $\Delta m_{\odot}^{2}$ (with $m_{2}>m_{1}$ ) has already been established by the oscillations in matter, as will be explained in section 2.4.1.

In the MINOS experiment, studied in this thesis, a beam of $\nu_{\mu}$ is produced and, after allowing for neutrino propagation, neutrinos of the same flavour are detected. The survival probability can be written, expanding equation (2.16):

$$
\begin{aligned}
P\left(\nu_{\mu} \rightarrow \nu_{\mu}\right)= & 1-4\left|U_{\mu 3}\right|^{2}\left|U_{\mu 1}\right|^{2} \sin ^{2} \Delta_{31} \\
& -4\left|U_{\mu 3}\right|^{2}\left|U_{\mu 2}\right|^{2} \sin ^{2} \Delta_{32}-4\left|U_{\mu 2}\right|^{2}\left|U_{\mu 1}\right|^{2} \sin ^{2} \Delta_{21}
\end{aligned}
$$

MINOS is designed to precisely measure $\left|\Delta m_{32}^{2}\right|$ but has no sensitivity to $\Delta m_{21}^{2}$ because of the ratio between the baseline and the neutrino energy chosen. Consequently the mass states $m_{1}$ and $m_{2}$ can be treated as degenerate. Equation (2.19) can be rewritten as:

$$
P\left(\nu_{\mu} \rightarrow \nu_{\mu}\right)=1-4\left|U_{\mu 3}\right|^{2}\left(1-\left|U_{\mu 3}\right|^{2}\right) \sin ^{2} \Delta_{\mu \mu}+\mathcal{O}\left(\Delta_{21}^{2}\right)
$$

where $\left|U_{\mu 3}\right|^{2}=c_{13}^{2} s_{23}^{2}$ and $\Delta_{\mu \mu}=\Delta m_{\mu \mu}^{2} L / 4 E . \Delta_{\mu \mu}$ is the effective atmospheric $\Delta m^{2}$ 
for the $\nu_{\mu}$ disappearance channel and is given by the muon flavour weighted average of the mass splittings:

$$
\Delta m_{\mu \mu}^{2}=\frac{\left|U_{\mu 1}\right|^{2}\left|\Delta m_{31}^{2}\right|+\left|U_{\mu 2}\right|^{2}\left|\Delta m_{32}^{2}\right|}{\left(\left|U_{\mu 1}\right|^{2}+\left|U_{\mu 2}\right|^{2}\right)}
$$

Allowing $\theta_{13}=0$, a further simplification can be made:

$$
\begin{gathered}
4\left|U_{\mu 3}\right|^{2}\left(1-\left|U_{\mu 3}\right|^{2}\right)=\sin ^{2}\left(2 \theta_{23}\right) \\
\Delta m_{\mu \mu}^{2}=s_{12}^{2}\left|\Delta m_{31}^{2}\right|+c_{12}^{2}\left|\Delta m_{32}^{2}\right|
\end{gathered}
$$

The difference between $\Delta m_{\mu \mu}^{2}$ and $\left|\Delta m_{32}^{2}\right|$ is considered negligible and all results will be quoted as a function of $\left|\Delta m_{32}^{2}\right|$ or $\Delta m_{\mathrm{atm}}^{2}$. Abandoning natural units in equation (2.19) and using the simplifications in equation (2.22), the survival probability $P\left(\nu_{\alpha} \rightarrow \nu_{\alpha}\right)$ in the case of the MINOS experiment $\left(P\left(\nu_{\mu} \rightarrow \nu_{\mu}\right)\right)$ can be written as:

$$
P\left(\nu_{\alpha} \rightarrow \nu_{\alpha}\right)=1-\sin ^{2}(2 \theta) \sin ^{2}\left(\frac{1.27 \Delta m^{2} L}{E}\right)
$$

$L$ is the distance travelled in $\mathrm{km}, E$ is the neutrino energy in $\mathrm{GeV}, \Delta m^{2}$ is measured in $\mathrm{eV}^{2}$. In the case of atmospheric neutrinos, $\Delta m^{2}$ corresponds to $\Delta m_{\mathrm{atm}}^{2}$ and the mixing angle $\theta$ corresponds to $\theta_{23}$. The probability from the two-flavour oscillation model $P\left(\nu_{\mu} \rightarrow \nu_{\mu}\right)$ (equation (2.24)) is shown in figure 2.2.

In the three-flavour neutrino model, the $\nu_{e}$ survival probability can be written as:

$$
\begin{aligned}
P\left(\nu_{e} \rightarrow \nu_{e}\right)= & 1-4\left|U_{e 3}\right|^{2}\left|U_{e 1}\right|^{2} \sin ^{2} \Delta_{31}-4\left|U_{e 3}\right|^{2}\left|U_{e 2}\right|^{2} \sin ^{2} \Delta_{32} \\
& -4\left|U_{e 2}\right|^{2}\left|U_{e 1}\right|^{2} \sin ^{2} \Delta_{21}
\end{aligned}
$$

Figure 2.3 shows the survival probability for the world average parameter values taken from [23] as given from equation (2.25). It is immediately possible to see that, if $\theta_{13}$ is non zero, the survival probability shows two different oscillation regimes: above $L / E \approx$ $15 \mathrm{~km} / \mathrm{MeV}$, corresponding to the solar $L / E$, and at $L / E \approx 0.5 \mathrm{~km} / \mathrm{MeV}$, corresponding 


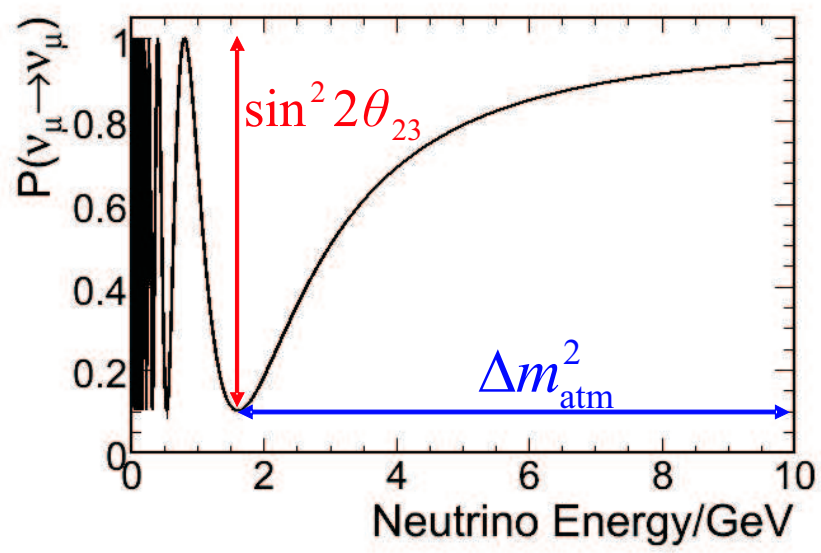

Figure 2.2: The survival probability $P\left(\nu_{\mu} \rightarrow \nu_{\mu}\right)$ in the two-flavour model as in equation (2.24) as a function of neutrino energy. The oscillation parameter $\Delta m_{\text {atm }}^{2}$ governs the horizontal position of the oscillation minimum, while the parameter $\sin ^{2}\left(2 \theta_{23}\right)$ governs the depth of the dip. At lower neutrino energy the oscillations become so rapid that experiments can only detect an average disappearance of $1 / 2$. The figure is taken from [22].

to the atmospheric $L / E$. The survival probability in the case of atmospheric $L / E$ can be written from equation (2.25) as:

$$
P\left(\nu_{e} \rightarrow \nu_{e}\right)=1-\sin ^{2}\left(2 \theta_{13}\right) \sin ^{2} \Delta_{e e}+\mathcal{O}\left(\Delta_{21}^{2}\right)
$$

where $\Delta_{e e}=\Delta m_{e e}^{2} L / 4 E$ and $\Delta m_{e e}^{2}$ is the effective atmospheric $\Delta m^{2}$ for the $\nu_{e}$ oscillation channel, in analogy with equation (2.23). As $\Delta_{21}^{2}$ is small compared to the atmospheric $\Delta_{e e}$, equation (2.26) can be reduced to the two-flavour oscillation formula (2.24). In the case of solar neutrino atmospheric oscillation, $\Delta m^{2}$ corresponds to $\Delta m_{e e}^{2}$ and the mixing angle $\theta$ corresponds to $\theta_{13}$.

The $\nu_{e}$ survival probability for the solar $L / E$ can be written from equation (2.25) as:

$$
P\left(\nu_{e} \rightarrow \nu_{e}\right)=c_{13}^{4}\left(1-\sin ^{2}\left(2 \theta_{12}\right) \sin ^{2} \Delta_{21}\right)+\frac{\sin ^{2}\left(2 \theta_{13}\right)}{2}
$$

where the only effect that $\theta_{13}$ has is a small perturbation on the survival probability. As $\theta_{13}$ can be approximated to 0 , it is possible to reduce equation (2.27) to the simplified formula in equation (2.24). In the case of solar $L / E, \Delta m^{2}$ corresponds to $\Delta m_{21}^{2}$ and the mixing 


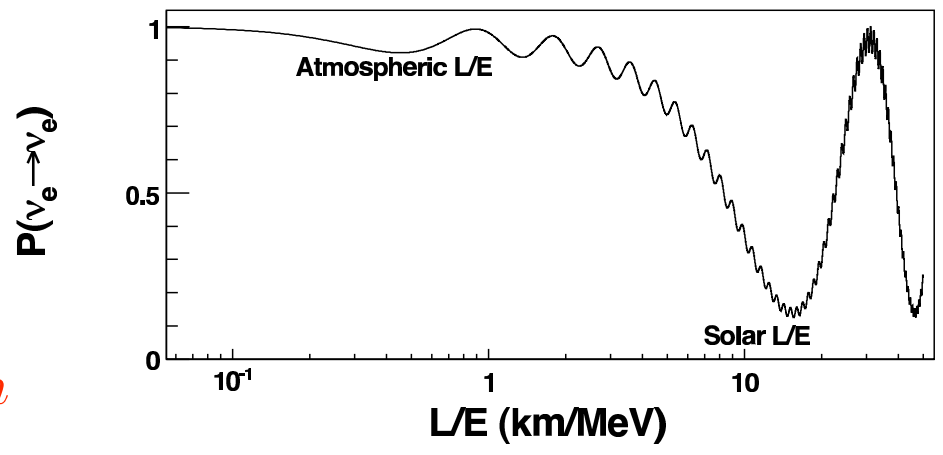

Figure 2.3: The figure shows the survival probability for $\nu_{e}$ as a function of $L / E$. The two different oscillation regimes a neutrino can undergo are shown: the atmospheric $L / E$ (corresponding to greater $\Delta m^{2}$ ) and the solar $L / E$ (corresponding to a smaller $\Delta m^{2}$ ). Figure is taken from [23].

angle $\theta$ corresponds to $\theta_{12}$.

The simplified version of the survival probability in equation (2.24) allows some general considerations: the oscillation maximum happens when $\theta=\pi / 4$ and

$$
1.27 \frac{\Delta m^{2} L}{E} \approx \frac{\pi}{2}
$$

Note that oscillations are only sensitive to the modulus of the $\Delta m^{2}$ parameter and cannot add any information on the neutrino mass hierarchy.

\subsubsection{Neutrino oscillations in matter}

So far, neutrinos were only considered to propagate in vacuum. When they propagate in matter, the electron neutrino plays an important role. While all flavor neutrinos are allowed a coherent forward scattering on an electron through a $Z^{0}$ boson exchange in neutral current interactions, $\nu_{e}$ are also allowed to scatter via a $W$ boson exchange in charged current interactions. Figure 2.4 shows the possible interactions for a neutrino scattering on an electron. The NC interaction, that is allowed for all flavours, is on the left; the $\mathrm{CC}$ interaction, that is allowed only for $\nu_{e}$, is on the right. Matter effects were studied by Wolfenstein in [24] and by Mikheyev and Smirnov in [25]. In the vacuum, in a two- 

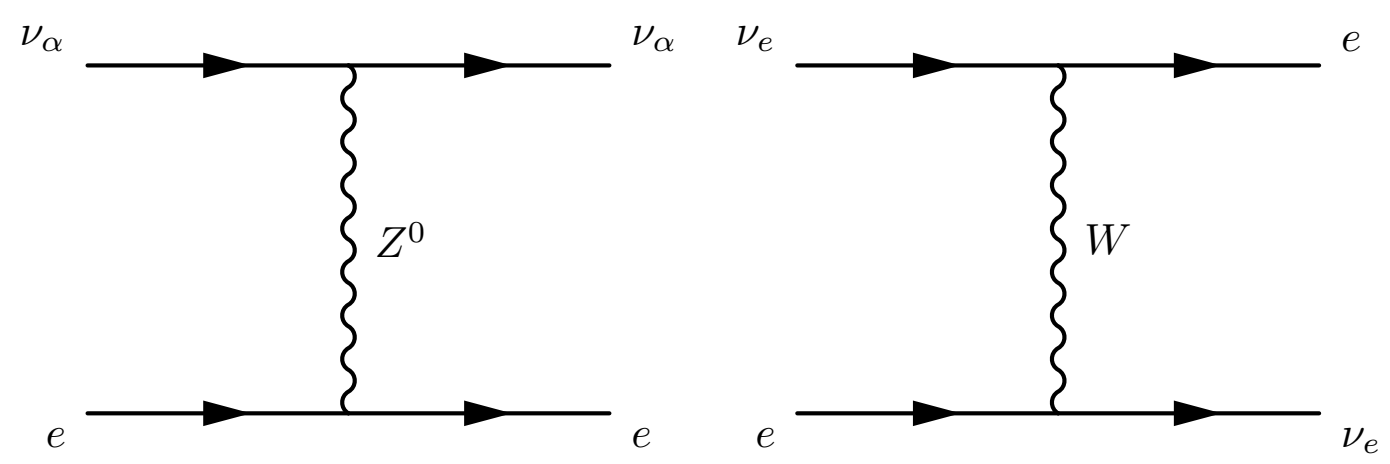

Figure 2.4: The diagrams show the two coherent forward scattering processes for neutrinos on electrons. The diagram on the left (NC) is allowed for all flavour neutrinos. The diagram on the right $(\mathrm{CC})$ is only allowed for $\nu_{e}$.

flavour formulation, the mass eigenstates are eigenstates of the free-particle Hamiltonian $\mathcal{H}_{0}:$

$$
\mathrm{i} \frac{\partial}{\partial t}\left(\begin{array}{c}
\nu_{1} \\
\nu_{2}
\end{array}\right)=\mathcal{H}_{0}\left(\begin{array}{c}
\nu_{1} \\
\nu_{2}
\end{array}\right) .
$$

Matter effects can greatly enhance neutrino mixing, resulting in a large oscillation probability even for small vacuum mixing angles. The extra contribution given by the $\nu_{e}$ appears in the Hamiltonian through an additional term $\sqrt{2} G_{F} N_{e}$, where $G_{F}$ is the Fermi constant and $N_{e}$ is the number density of electrons in matter. The matter-induced potentials are given by:

$$
\begin{aligned}
& V_{e}=\sqrt{2} G_{F}\left(N_{e}-\frac{N_{n}}{2}\right)=V_{e}^{C C}+V^{N C} \\
& V_{\mu}=V_{\tau}=-\sqrt{2} G_{F} \frac{N_{n}}{2}=V^{N C}
\end{aligned}
$$

where $N_{n}$ is the neutron number density.

The time evolution in matter can be written as:

$$
\mathrm{i} \frac{\partial}{\partial t} U^{\dagger}\left(\begin{array}{c}
\nu_{e} \\
\nu_{x}
\end{array}\right)=\mathcal{H} U^{\dagger}\left(\begin{array}{c}
\nu_{e} \\
\nu_{x}
\end{array}\right)
$$

where $\mathcal{H}$ represents the Hamiltonian, evaluated as the sum of the free particle Hamiltonian 
$\mathcal{H}_{0}$ plus a potential term $V$ (if the propagation is in matter). $U$ is the rotation matrix between the mass and flavour eigenstates in the vacuum, given by:

$$
U=\left(\begin{array}{cc}
\cos \theta & \sin \theta \\
-\sin \theta & \cos \theta
\end{array}\right) \text {. }
$$

Assuming the neutrino mass is small, the free particle Hamiltonian can be written as:

$$
\mathcal{H}_{0} \nu_{i}=E \nu_{i}=\left(\sqrt{p^{2}+m_{i}^{2}}\right) \nu_{i} \approx\left(p+\frac{m_{i}^{2}}{2 p}\right) \nu_{i} \approx\left(p+\frac{m_{i}^{2}}{2 E}\right) \nu_{i}
$$

Substituting the free particle Hamiltonian and the potential expressions, equation (2.31) becomes:

$$
\begin{aligned}
\mathrm{i} \frac{\partial}{\partial t} U^{\dagger}\left(\begin{array}{c}
\nu_{e} \\
\nu_{x}
\end{array}\right) & =\left[p \mathbb{1}+\left(\begin{array}{cc}
\frac{m_{1}^{2}}{2 E} & 0 \\
0 & \frac{m_{2}^{2}}{2 E}
\end{array}\right)+\left(\begin{array}{cc}
V_{e} & 0 \\
0 & V_{x}
\end{array}\right)\right] U^{\dagger}\left(\begin{array}{c}
\nu_{e} \\
\nu_{x}
\end{array}\right) \\
& =\left[\left(p+\frac{m_{1}^{2}}{2 E}\right) \mathbb{1}+\left(\begin{array}{cc}
0 & 0 \\
0 & \frac{\Delta m_{21}^{2}}{2 E}
\end{array}\right)+\left(\begin{array}{cc}
V_{e} & 0 \\
0 & V_{x}
\end{array}\right)\right] U^{\dagger}\left(\begin{array}{c}
\nu_{e} \\
\nu_{x}
\end{array}\right)
\end{aligned}
$$

Equation (2.34), after being multiplied by $U$ from the left and after inserting the expressions for the potentials in equation (2.30), becomes:

$$
\begin{aligned}
\mathrm{i} \frac{\partial}{\partial t}\left(\begin{array}{c}
\nu_{e} \\
\nu_{x}
\end{array}\right) & =\left[\left(p+\frac{m_{1}^{2}}{2 E}\right) \mathbb{1}+U\left(\begin{array}{cc}
0 & 0 \\
0 & \frac{\Delta m_{21}^{2}}{2 E}
\end{array}\right) U^{\dagger}+\left(\begin{array}{cc}
V_{e} & 0 \\
0 & V_{x}
\end{array}\right)\right]\left(\begin{array}{c}
\nu_{e} \\
\nu_{x}
\end{array}\right) \\
& =\left(p+\frac{m_{1}^{2}}{2 E}+1+V^{N C}\right) \mathbb{1}\left(\begin{array}{c}
\nu_{e} \\
\nu_{x}
\end{array}\right) \\
& +\left[\left(\begin{array}{cc}
-\cos (2 \theta) & \sin (2 \theta) \\
\sin (2 \theta) & \cos (2 \theta)
\end{array}\right) \frac{\Delta m_{21}^{2}}{4 E}+\left(\begin{array}{cc}
V_{e}^{C C} & 0 \\
0 & 0
\end{array}\right)\right]\left(\begin{array}{c}
\nu_{e} \\
\nu_{x}
\end{array}\right)
\end{aligned}
$$

Collecting all diagonal terms into a constant $K$ and substituting the potential terms in 
equation (2.30):

$$
\begin{aligned}
\mathrm{i} \frac{\partial}{\partial t}\left(\begin{array}{c}
\nu_{e} \\
\nu_{x}
\end{array}\right) & =K \mathbb{1}\left(\begin{array}{c}
\nu_{e} \\
\nu_{x}
\end{array}\right) \\
& +\left(\begin{array}{cc}
-\cos (2 \theta)-\frac{G_{F} N_{e} 4 E}{\Delta m_{21}^{2} \sqrt{2}} & \sin (2 \theta) \\
\sin (2 \theta) & \cos (2 \theta)+\frac{G_{F} N_{e} 4 E}{\Delta m_{21}^{2} \sqrt{2}}
\end{array}\right) \frac{\Delta m_{21}^{2}}{4 E}\left(\begin{array}{c}
\nu_{e} \\
\nu_{x}
\end{array}\right)
\end{aligned}
$$

It is possible to find a transformation matrix such that the matrix in equation (2.36) is made diagonal:

$$
\left(\begin{array}{cc}
\cos \theta_{m} & \sin \theta_{m} \\
-\sin \theta_{m} & \cos \theta_{m}
\end{array}\right)\left(\begin{array}{cc}
-\Lambda_{m} & 0 \\
0 & \Lambda_{m}
\end{array}\right)\left(\begin{array}{cc}
\cos \theta_{m} & -\sin \theta_{m} \\
\sin \theta_{m} & \cos \theta_{m}
\end{array}\right)
$$

where:

$$
\begin{aligned}
\sin \left(2 \theta_{m}\right) & =\frac{\sin (2 \theta)}{\sqrt{\left(\cos (2 \theta)-\frac{2 E \sqrt{2} G_{F} N_{e}}{\Delta m_{21}^{2}}\right)^{2}+\sin ^{2}(2 \theta)}} \\
\Lambda_{m} & =\Delta m_{21}^{2} \frac{\sin (2 \theta)}{\sin \left(2 \theta_{m}\right)}=\Delta m_{21}^{2} \sqrt{\left(\cos (2 \theta)-\frac{2 E \sqrt{2} G_{F} N_{e}}{\Delta m_{21}^{2}}\right)^{2}+\sin ^{2}(2 \theta)}
\end{aligned}
$$

In the same fashion as shown in the case of the propagation in the vacuum, the probability of oscillation can be written as:

$$
P\left(\nu_{\alpha} \rightarrow \nu_{\beta}\right)=\sin ^{2}\left(2 \theta_{m}\right) \sin ^{2}\left(\frac{\Lambda_{m} L}{4 E}\right)
$$

where the effective parameters $\Lambda_{m}$ and $\theta_{m}$ have been introduced. Matter effects are important because:

- The effective mixing can be maximal $\left(\sin ^{2}\left(2 \theta_{m}\right)=1\right)$ even at small vacuum mixing angles. This is due to the resonance condition in equation $(2.38)$ when $\cos (2 \theta)=$ 
$\left(2 E \sqrt{2} G_{F} N_{e}\right) / \Delta m_{21}^{2}$, that is known as the MSW resonance (after Mikheyev-SmirnovWolfenstein).

- The oscillation probability loses its symmetry in $\theta$ about $\pi / 4$ due to the $\cos (2 \theta)$ term.

- Except at the maximal mixing given by $\sin ^{2}(2 \theta)=1$, the oscillation probability depends on the sign of $\Delta m^{2}$, giving sensitivity to the mass ordering. For the resonant condition to occur it is necessary that $\Delta m_{21}^{2}>0$, bounding $m_{2}>m_{1}$.

- The potential energy for neutrinos and antineutrinos has a different sign: the matter effect resonant condition could occur either for neutrinos or antineutrinos. This asymmetry could be exploited to determine the neutrino mass hierarchy from atmospheric neutrinos, as suggested in [26], by studying the transition $\nu_{e} \rightarrow \nu_{\mu}$ and $\bar{\nu}_{e} \rightarrow \bar{\nu}_{\mu}$ that could be resonant in matter.

\subsubsection{Oscillation into sterile neutrinos}

The measurement of the width of the $Z^{0}$ boson bounds the number of light active neutrinos to three. Nevertheless, as some experiments have suggested, some additional types of neutrinos may not couple with the $Z^{0}$ boson and are referred as "sterile". In this section the formalism for four neutrino mass states is given.

The mixing between the three active neutrino flavours and one sterile neutrino requires the addition of one mass eigenstate, expanding the PMNS mixing matrix in equation (2.17) to a $4 \times 4$ matrix. The expanded mixing matrix contains six mixing angles and six phases, with three of the phases being Majorana phases that are not relevant to oscillation experiments. The matrix can be written as a product of six independent rotation matrices about the Euler axes $R_{i j}$, where $i j$ refers to the plane in which a particular rotation takes place, following the formalism in [27].

As MINOS is designed to precisely measure $\left|\Delta m_{32}^{2}\right|$ but has no sensitivity to $\Delta m_{21}^{2}$, the mass states $m_{1}$ and $m_{2}$ can be treated as degenerate. As a consequence, the corresponding 
rotation matrix in $\theta_{12}$ vanishes from the oscillation probabilities when it is the rightmost matrix. As in the standard formulation of the matrix (2.17), the chosen formalism for the extended matrix couples one of the Dirac CP violating phases, $\delta_{1}$ to $\theta_{13}$. Furthermore, in the assumption the mass eigenstates $\nu_{1}$ and $\nu_{2}$ are degenerate, the Dirac CP violating phase $\delta_{3}$ is eliminated. The general form of the mixing matrix used by the current MINOS analysis is written as:

$$
\begin{aligned}
U & =R_{34}\left(\theta_{34}\right) R_{24}\left(\theta_{24}, \delta_{2}\right) R_{14}\left(\theta_{14}\right) R_{23}\left(\theta_{23}\right) R_{13}\left(\theta_{13}, \delta_{1}\right) R_{12}\left(\theta_{12}, \delta_{3}\right), \\
& =R_{34}\left(\theta_{34}\right) R_{24}\left(\theta_{24}, \delta_{2}\right) R_{14}\left(\theta_{14}\right) R_{23}\left(\theta_{23}\right) R_{13}\left(\theta_{13}, \delta_{1}\right)
\end{aligned}
$$

where the $\delta_{1}, \delta_{2}$ and $\delta_{3}$ are the three CP violating Dirac phases and the last equality reflects the assumption of degeneracy in mass states 1 and 2. Thus, the mixing matrix can be written as,

$$
U=\left[\begin{array}{cccc}
U_{e 1} & U_{e 2} & c_{14} s_{13} e^{-i \delta_{1}} & s_{14} \\
U_{\mu 1} & U_{\mu 2} & -s_{14} s_{13} e^{-i \delta_{1}} s_{24} e^{-i \delta_{2}}+c_{13} s_{23} c_{24} & c_{14} s_{24} e^{-i \delta_{2}} \\
U_{\tau 1} & U_{\tau 2} & -s_{14} c_{24} s_{34} s_{13} e^{-i \delta_{1}}-c_{13} s_{23} s_{34} s_{24} e^{i \delta_{2}}+c_{13} c_{23} c_{34} & c_{14} c_{24} s_{34} \\
U_{s 1} & U_{s 2} & -s_{14} c_{24} c_{34} s_{13} e^{-i \delta_{1}}-c_{13} s_{23} c_{34} s_{24} e^{i \delta_{2}}-c_{13} c_{23} s_{34} & c_{14} c_{24} c_{34}
\end{array}\right]
$$

Equation (2.16) can be expanded for the different oscillation scenarios:

$$
\begin{aligned}
P_{\nu_{\mu} \rightarrow \nu_{\mu}}= & 1-4\left\{\left|U_{\mu 3}\right|^{2}\left(1-\left|U_{\mu 3}\right|^{2}-\left|U_{\mu 4}\right|^{2}\right) \sin ^{2} \Delta_{31}+\left|U_{\mu 4}\right|^{2}\left|U_{\mu 3}\right|^{2} \sin ^{2} \Delta_{43}\right. \\
& \left.+\left|U_{\mu 4}\right|^{2}\left(1-\left|U_{\mu 3}\right|^{2}-\left|U_{\mu 4}\right|^{2}\right) \sin ^{2} \Delta_{41}\right\} \\
P_{\nu_{\mu} \rightarrow \nu_{\alpha}}= & 4 \Re \mathfrak{R e}\left\{\left|U_{\mu 3}\right|^{2}\left|U_{\alpha 3}\right|^{2} \sin ^{2} \Delta_{31}+\left|U_{\mu 4}\right|^{2}\left|U_{\alpha 4}\right|^{2} \sin ^{2} \Delta_{41}\right. \\
& \left.+U_{\mu 4}^{*} U_{\alpha 4} U_{\mu 3} U_{\alpha 3}^{*}\left(\sin ^{2} \Delta_{31}-\sin ^{2} \Delta_{43}+\sin ^{2} \Delta_{41}\right)\right\} \\
& +2 \mathfrak{I m}\left\{U_{\mu 4}^{*} U_{\alpha 4} U_{\mu 3} U_{\alpha 3}^{*}\left(\sin 2 \Delta_{31}-\sin 2 \Delta_{41}+\sin 2 \Delta_{43}\right)\right\}
\end{aligned}
$$


where $\alpha=e, \tau$, or $s$ (where $s$ is for sterile neutrino) and the orthogonality constraint $\sum_{i} U_{a i} U_{b i}^{*}=0$ has been used to eliminate the matrix elements corresponding to the first and second mass states. The following simplifying assumptions are made to reduce the number of possible parameters:

- A CP violating phase is removed by setting $\delta_{2}=0$.

- The angle $\theta_{14}$ is set to $0^{\circ}$ to remove one parameter, as it is known from the literature to be small [28].

- $\theta_{13}$ is eliminated as a free parameter by only considering two values, $\theta_{13}=0^{\circ}$ or $\theta_{13}=12^{\circ}$, where the latter is the CHOOZ limit [29] at the MINOS measured value of $\left|\Delta m_{32}^{2}\right|$

- The remaining $\mathrm{CP}$ violating phase, $\delta_{1}$, is taken to be $3 \pi / 2$ as this value maximises the $\nu_{e}$ appearance probability at MINOS $\left|\Delta m^{2}\right|[30]$.

There are many possible models to integrate the fourth mass state $m_{4}$. Experimental results have ruled out models in which $m_{4}$ is degenerate with one of the active neutrino mass states [31]. If the $m_{4}$ mass state is degenerate with $m_{3}$, the model is often referred to as the $2+2$ model. In this case the sterile mass splitting would correspond to the atmospheric mass splitting. There is still interest in the literature $([28,27,31])$ in models in which $m_{4} \gg m_{3}$, i.e. the sterile mass splitting is much higher than the solar and atmospheric mass splitting. Figure 2.5 shows the mass ordering in case of normal hierarchy for this $3+1$ neutrino model. The model has also validity for the inverted hierarchy case. Results from this sterile neutrino model in MINOS are studied in this thesis and in [30]. As the splitting involving $m_{4}$ is much larger than the others, then from an experimental point of view the $\sin ^{2} \Delta_{41}$ and $\sin ^{2} \Delta_{43}$ oscillations will be rapid compared to the resolution in $L / E$ and the probabilities measured will simply be $1 / 2$. For the same reason, $\sin \left(2 \Delta_{41}\right)$ and $\sin \left(2 \Delta_{43}\right)$ average to 0. This analysis assumes no oscillation at the MINOS Near Detector, limiting 


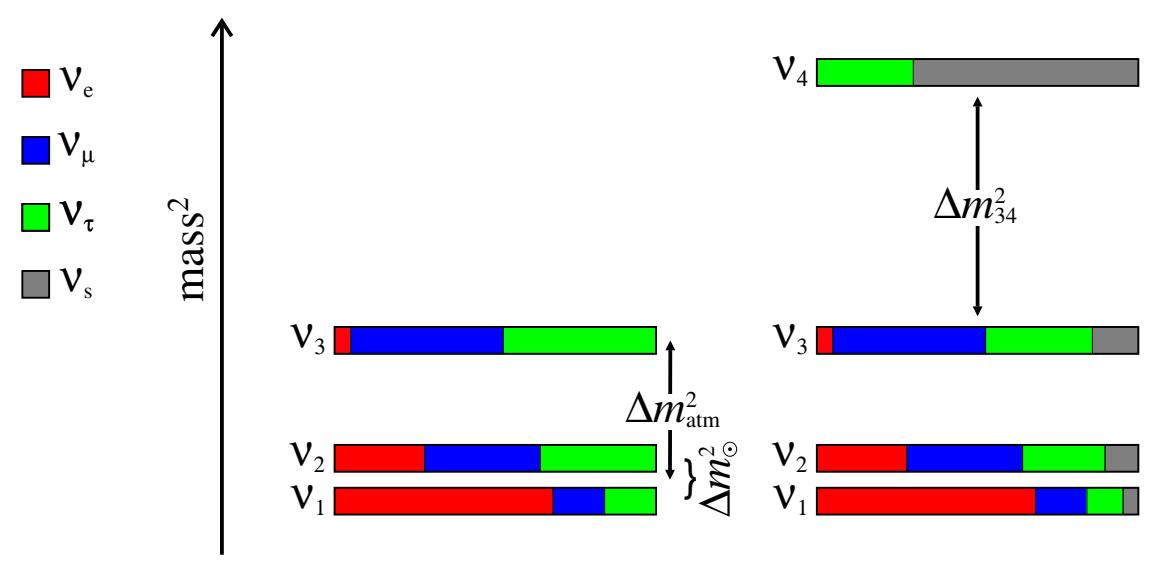

Figure 2.5: The figure shows a possible neutrino mass ordering in case of a fourth mass state associated to a sterile neutrino. This model, often referred to as $3+1$ as the fourth mass state is at higher mass splitting, will be studied in the course of this thesis. The figure is taken from [21].

the value of $\left|\Delta m_{43}^{2}\right|$ to $<1 \mathrm{eV}^{2}$. With all these simplifications, the probabilities become:

$$
\begin{aligned}
P\left(\nu_{\mu} \rightarrow \nu_{\mu}\right)= & 1-4 c_{24}^{4} c_{13}^{2} s_{23}^{2}\left(1-c_{13}^{2} s_{23}^{2}\right) \sin ^{2}\left(1.27 \Delta m_{31}^{2} L / E\right)+\frac{1}{2} \sin ^{2}\left(2 \theta_{24}\right) \\
P\left(\nu_{\mu} \rightarrow \nu_{e}\right)= & s_{23}^{2} c_{24}^{2} \sin ^{2}\left(2 \theta_{13}\right) \sin ^{2}\left(1.27 \Delta m_{31}^{2} L / E\right) \\
P\left(\nu_{\mu} \rightarrow \nu_{\tau}\right)= & \frac{1}{2} s_{34}^{2} \sin ^{2}\left(2 \theta_{24}\right)+\left(c_{13}^{4} s_{23}^{4} s_{34}^{2} \sin ^{2}\left(2 \theta_{24}\right)+c_{13}^{4} c_{24}^{2} c_{34}^{2} \sin ^{2}\left(2 \theta_{23}\right)\right. \\
& -c_{13}^{4} s_{23}^{2} c_{24} \sin \left(2 \theta_{24}\right) \sin \left(2 \theta_{23}\right) \sin \left(2 \theta_{34}\right)-c_{13}^{2} s_{23}^{2} s_{34}^{2} \sin ^{2}\left(2 \theta_{24}\right) \\
& \left.+\frac{1}{2} c_{13}^{2} c_{24} \sin \left(2 \theta_{24}\right) \sin \left(2 \theta_{23}\right) \sin \left(2 \theta_{34}\right)\right) \sin ^{2}\left(1.27 \Delta m_{31}^{2} L / E\right) \\
P\left(\nu_{\mu} \rightarrow \nu_{s}\right)= & \frac{1}{2} c_{34}^{2} \sin ^{2}\left(2 \theta_{24}\right)+\left(c_{13}^{4} s_{23}^{4} c_{34}^{2} \sin { }^{2}\left(2 \theta_{24}\right)+c_{13}^{4} c_{24}^{2} s_{34}^{2} \sin ^{2}\left(2 \theta_{23}\right)\right. \\
& +c_{13}^{4} s_{23}^{2} c_{24} \sin \left(2 \theta_{24}\right) \sin \left(2 \theta_{23}\right) \sin \left(2 \theta_{34}\right)-c_{13}^{2} s_{23}^{2} c_{34}^{2} \sin ^{2}\left(2 \theta_{24}\right) \\
& \left.-\frac{1}{2} c_{13}^{2} c_{24} \sin \left(2 \theta_{23}\right) \sin \left(2 \theta_{24}\right) \sin \left(2 \theta_{34}\right)\right) \sin ^{2}\left(1.27 \Delta m_{31}^{2} L / E\right)
\end{aligned}
$$

The probabilities depend on three angle parameters, $\theta_{23}, \theta_{24}$ and $\theta_{34}$ and one mass splitting parameter $\left|\Delta m_{31}^{2}\right|$.

Some experimental constraints can reduce the allowed ranges for the angles. Following the arguments in [28], the probability $P\left(\nu_{e} \rightarrow \nu_{e}\right)$ can be written as:

$$
P\left(\nu_{e} \rightarrow \nu_{e}\right)=1-\sin ^{2}\left(2 \theta_{14}\right) \sin ^{2} \Delta_{41}-c_{14}^{4} \sin ^{2}\left(2 \theta_{13}\right) \sin ^{2} \Delta_{31}
$$



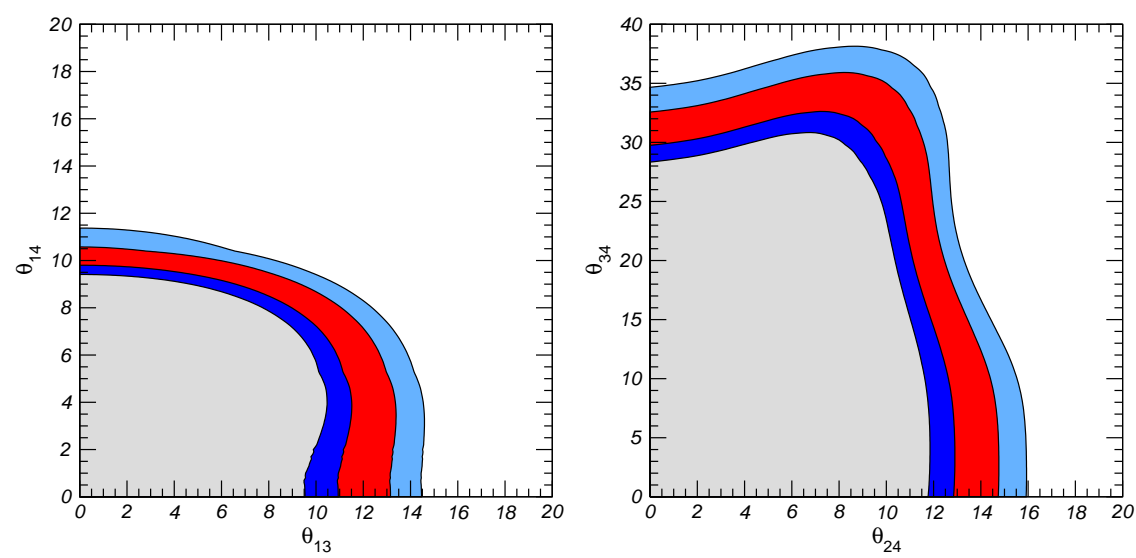

Figure 2.6: The figure shows the allowed angle ranges for the angles in the sterile neutrino model considered. In particular the $90 \%, 95 \%, 99 \%$ and $3 \sigma$ C.L. are showns in the $\left(\theta_{13}, \theta_{14}\right)$ plane on the left and in the $\left(\theta_{24}, \theta_{34}\right)$ plane on the right. The angles are expressed in degrees. Figure taken from [28].

Reactor experiments like Bugey and CHOOZ give constraints on the $\theta_{14}$ and $\theta_{13}$ angles in equation (2.45): both angles are bounded to be less than $14^{\circ}$. It needs to be noted that in the formulation in [28] the angle $\theta_{14}$ is not fixed to 0. Experiments on atmospheric neutrinos measure the probability $P\left(\nu_{\mu} \rightarrow \nu_{\mu}\right)$ in equation (2.44). Such experiments bound the allowed ranges for the angle $\theta_{24}$, that is around $14^{\circ}$ as well. Figure 2.6 shows the allowed ranges for four angles as obtained from combining experimental results. In the analysis presented in this thesis, it has been decided to conservatively allow the angle ranges to be: $\theta_{24}<60^{\circ}, \theta_{34}<60^{\circ}$ and $\theta_{23}<90^{\circ}$.

\section{$2.4 \quad$ Experimental evidence}

\subsubsection{Solar neutrinos}

The nuclear fusion processes happening in the Sun make it an intense source of electron neutrinos, with a flux of $\approx 6 \times 10^{10} / \mathrm{cm}^{2} / \mathrm{s}$. The main process in the Solar Standard Model (SSM) is the $p p$ chain, the main part of which is:

$$
4 p \rightarrow{ }^{4} \mathrm{He}+2 e^{-}+2 \nu_{e}+26.73 \mathrm{MeV} .
$$




\begin{tabular}{llc}
\hline \hline Reaction & Abbr. & Flux $\left(\mathrm{cm}^{-2} \mathrm{~s}^{-1}\right)$ \\
\hline$p p \rightarrow d e^{+} \nu$ & $p p$ & $5.99(1.00 \pm 0.01) \times 10^{10}$ \\
$p e^{-} p \rightarrow d \nu$ & $p e p$ & $1.42(1.00 \pm 0.02) \times 10^{8}$ \\
${ }^{3} \mathrm{He} p \rightarrow{ }^{4} \mathrm{He} e^{+}$ & $h e p$ & $7.93(1.00 \pm 0.16) \times 10^{3}$ \\
${ }^{7} \mathrm{Be} e^{-} \rightarrow{ }^{7} \mathrm{Li} \nu+(\gamma)$ & ${ }^{7} \mathrm{Be}$ & $4.84(1.00 \pm 0.11) \times 10^{9}$ \\
${ }^{8} \mathrm{~B} \rightarrow{ }^{8} \mathrm{Be}^{*} e^{+} \nu$ & ${ }^{8} \mathrm{~B}$ & $5.69(1.00 \pm 0.16) \times 10^{6}$ \\
\hline${ }^{13} \mathrm{~N} \rightarrow{ }^{13} \mathrm{C} e^{+} \nu$ & ${ }^{13} \mathrm{~N}$ & $3.07\left(1.00_{-0.28}^{+0.31}\right) \times 10^{8}$ \\
${ }^{15} \mathrm{O} \rightarrow{ }^{15} \mathrm{~N} e^{+} \nu$ & ${ }^{15} \mathrm{O}$ & $2.33\left(1.00_{-0.29}^{+0.33}\right) \times 10^{8}$ \\
${ }^{17} \mathrm{~F} \rightarrow{ }^{17} \mathrm{O} e^{+} \nu$ & ${ }^{17} \mathrm{~F}$ & $5.84(1.00 \pm 0.52) \times 10^{6}$ \\
\hline \hline
\end{tabular}

Table 2.2: Solar reactions producing neutrinos. The abbreviation name and the predicted flux is also given. The first five rows are from the $p p$ chain. The table is taken from [12].

Other interactions which are not on the $p p$ chain also produce neutrinos, but their contribution is limited to $2 \%$ of emitted neutrinos. Table 2.2 shows the interactions happening in the Sun that produce neutrinos. Figure 2.7 shows the corresponding neutrino energy spectrum. It should be noted that the solar neutrino energies overlap with energies of neutrinos from natural radioactivity. Having a good handle on natural radioactivity background is essential in solar neutrino experiments.

\section{Radiochemical experiments}

The first hint for neutrino oscillations came from solar neutrinos detected in radiochemical experiments. In 1968, Davis and collaborators published results from the Homestake chlorine experiment [32], showing that the measured flux of the solar electron neutrinos $\left({ }^{8} \mathrm{~B}\right.$ mainly) was lower than expected. The neutrino reaction studied by their experiment was:

$$
\nu_{e}+{ }^{37} \mathrm{Cl} \rightarrow e^{-}+{ }^{37} \mathrm{Ar}
$$

which has a threshold of $814 \mathrm{keV}$. The produced ${ }^{37} \mathrm{Ar}$ was then extracted and its rate of decay was recorded. Two other radiochemical experiments, SAGE and GALLEX, confirmed 


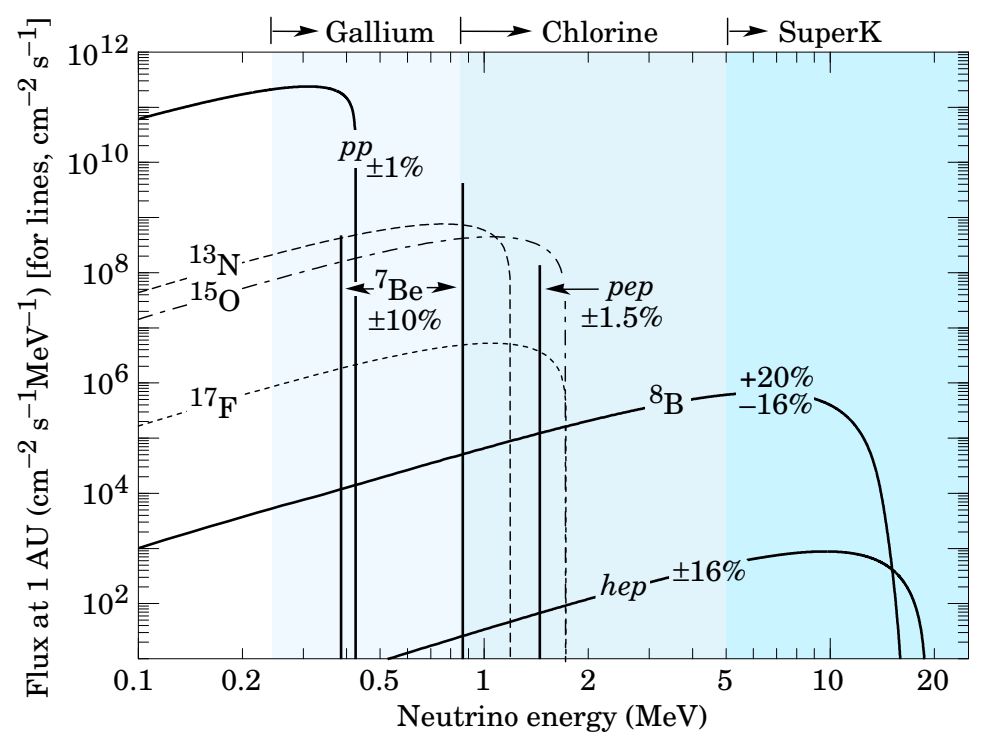

Figure 2.7: The solar neutrino fluxes and energy spectra predicted by the SSM. The figure is taken from [12]. The colour scheme corresponds to the energies studied in the three different types of experiments shown at the top.

the lower flux measurement using ${ }^{71} \mathrm{Ga}$. The neutrino reaction studied was:

$$
\nu_{e}+{ }^{71} \mathrm{Ga} \rightarrow e^{-}+{ }^{71} \mathrm{Ge}
$$

which has an end point of $223 \mathrm{keV}$. This lower end point makes it possible to study neutrinos coming from ${ }^{7} \mathrm{Be}$ and $p p$ neutrinos. Both the experimental results, GALLEX in 1992 [33, 34] and SAGE in 1991 [35, 36], show that nearly half of the expected electron neutrinos are missing. The GALLEX experiment was upgraded to become the GNO experiment, confirming the same results [37].

\section{Čerenkov detector experiments}

While the SAGE and GALLEX radiochemical experiments were sensitive to lower energy neutrinos, a Čerenkov detector experiment, Kamiokande, was taking data coming from the ${ }^{8} \mathrm{~B}$ neutrinos. Note that, even though the flux of ${ }^{8} \mathrm{~B}$ is lower than the flux of $p p$ neutrinos, the cross-section for neutrino interactions is proportional to the neutrino energy, making possible the detection of neutrinos from ${ }^{8} \mathrm{~B}$. The experiment is not sensitive to lower energy 
neutrinos because the natural radioactivity background is too high. The reaction studied was the elastic scattering $(\mathrm{ES})$ of $\nu_{e}$ on electrons:

$$
\nu_{e}+e^{-} \rightarrow \nu_{e}+e^{-}
$$

Using a Čerenkov detector is an advantage compared to the radiochemical experiments as it can give information about the rate, direction and kinematics of the neutrino events, while radiochemical experiments are only event counters. The outgoing $e^{-}$direction from elastic scattering is correlated to the direction of the neutrinos from the Sun. Kamiokande confirmed the deficit of solar neutrinos [38] and the similar, but much larger Super-Kamiokande (SK), recently measured the same flux [39].

Another Čerenkov detector experiment, SNO, consisted of a sphere of heavy water $\left(\mathrm{D}_{2} \mathrm{O}\right)$ surrounded by photomultipliers. While all the other experiments seen so far were only sensitive to reaction induced by $\nu_{e}$, the great advantage of SNO was that it was sensitive to three types of interaction:

$$
\begin{aligned}
& \nu_{e}+d \rightarrow p+p+e^{-}(\text {threshold }=1.4 \mathrm{MeV}) \\
& \nu_{x}+d \rightarrow n+p+\nu_{x}(\text { threshold }=2.2 \mathrm{MeV})
\end{aligned}
$$

as well as the electron scattering interaction (ES) like in Kamiokande and SK. The electron scattering interaction can happen for all neutrino flavours through neutral current interactions, and only to $\nu_{e}$ for charged current interactions. The $\nu_{e}$ interactions dominate this channel by a factor of $\approx 6$. From previous experiments it was clear the $\nu_{e}$ were disappearing and SNO's capability to detect this effect is through the (CC) interactions. The extra capability that SNO had is the ability to detect the rate of (NC) interactions. The neutral current interaction rate, if consistent with what was expected from the solar model would guarantee that $\nu_{e}$ have converted into other flavour neutrinos. Figure 2.8 shows the measured fluxes for $\nu_{e}\left(\phi_{e}\right)$ and non- $\nu_{e}$ neutrinos $\left(\phi_{\mu \tau}\right)$. The coloured bands show the $1 \sigma$ 


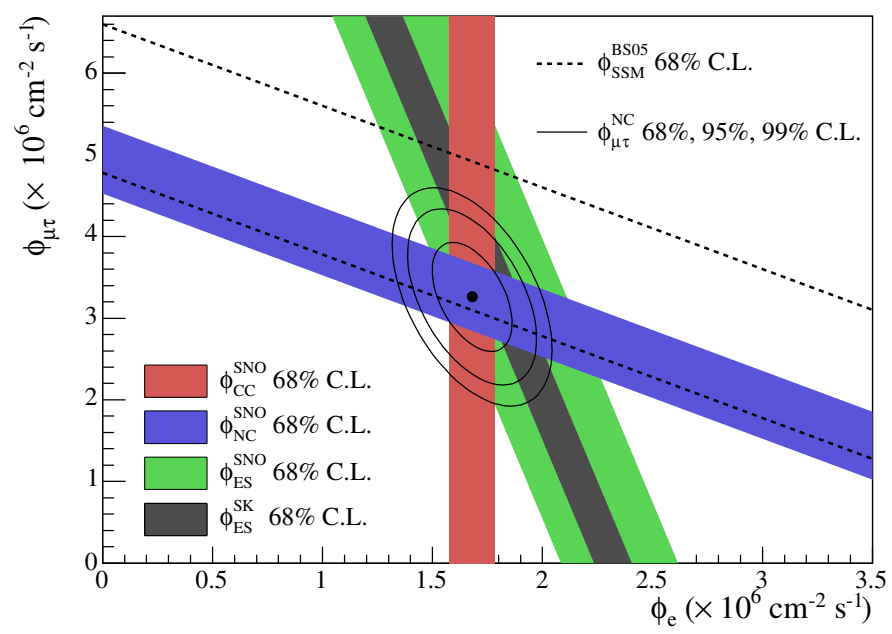

Figure 2.8: The flux $(\phi)$ of $\nu_{e}$ and $\nu_{\mu}+\nu_{\tau}$ from the Sun measured with the SNO detector. Dotted lines: region in which the $\nu_{\tau}+\nu_{\mu}+\nu_{e}$ fluxes sum to the SSM prediction. Blue: region in which the $\nu_{\tau}+\nu_{\mu}+\nu_{e}$ fluxes sum to the total flux measured from the NC interactions. Red: measured $\nu_{e}$ flux through the CC interaction with deuterium nuclei. Green: electron scattering measurement ( $\mathrm{NC}$ and $\mathrm{CC}$, but with $\mathrm{CC}$ dominating); grey: the same electron scattering measurement from the Super-Kamiokande experiment. Figure from [40].

uncertainty for each of the three interaction types. The bands showing the fluxes have different gradients due to the different sensitivities to non-electron neutrinos. There are two conclusions to be taken away from the charged current and neutral current measurements on this figure: comparing the overall ${ }^{8} \mathrm{~B}$ flux $\left(\phi_{e}+\phi_{\mu \tau}\right)$ with the SSM flux prediction they agree within errors, confirming that the SSM predictions are right. Furthermore, the fact that at the measured $\phi_{e}, \phi_{\mu \tau}$ is non zero shows that ${ }^{8} \mathrm{~B}$ neutrinos from the Sun convert into a combination of $\nu_{\mu}$ and $\nu_{\tau}$. The fact that the ES flux agrees with the others and with SK is a confirmation of the validity of the data.

\section{Liquid scintillator experiments}

Under the assumption of CPT conservation, equation (2.18) suggests another way it is possible to study the solar oscillation parameters through the survival probability $P\left(\bar{\nu}_{e} \rightarrow\right.$ $\left.\bar{\nu}_{e}\right)=P\left(\nu_{e} \rightarrow \nu_{e}\right)$ 


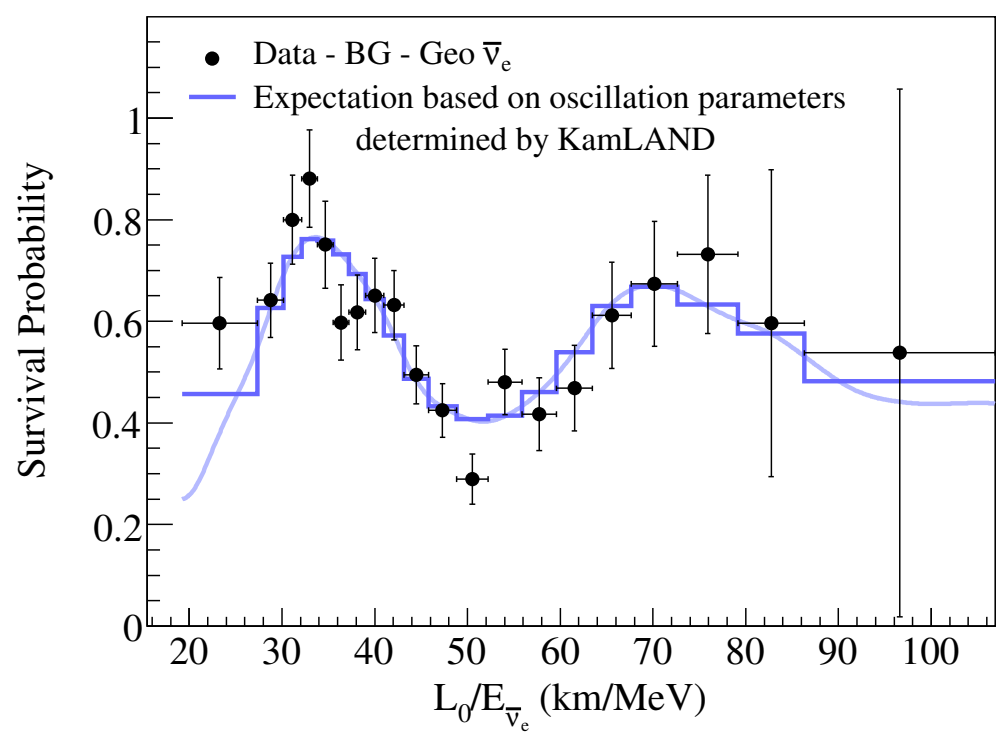

Figure 2.9: KamLAND oscillation results. The ratio of measured to expected $\bar{\nu}_{e}$ flux is shown (black dots) as a function of distance travelled divided by neutrino energy. The best fit of the oscillation hypothesis is shown in blue. KamLAND is the only experiment so far that has been able to measure two oscillation cycles. Figure taken from [12].

Reactor neutrino experiments study the reaction:

$$
\bar{\nu}_{e}+p \rightarrow e^{+}+n
$$

which is characterised by a prompt signal given by the positron annihilation and by a delayed signal given by the neutron capture. The experimental challenges of the experiment are given by the reduction of the natural radioactivity background and by a suitable target for the neutron capture.

The reactor experiment KamLAND, studied $P\left(\bar{\nu}_{e} \rightarrow \bar{\nu}_{e}\right)$ at the solar $L / E$, from equation (2.27), providing information on the parameters $\Delta m_{21}^{2}$ and $\theta_{12}$. KamLAND provides the world best measurement for $\Delta m_{21}^{2}=7.58_{-0.13}^{+0.14}(\text { stat })_{-0.15}^{+0.15}$ (syst) $\times 10^{-5} \mathrm{eV}^{2}$ [41]. The angle parameter was found to be: $\tan ^{2} \theta_{12}=0.56_{-0.07}^{+0.10}(\text { stat })_{-0.06}^{+0.10}($ syst $)$ [41]. Figure 2.9 shows the survival probability as measured by KamLAND as a function of $L / E$. The expected survival probability is also superimposed. For the first time two cycles of the oscillation periodic features are measured. 
Returning to solar neutrinos, the ${ }^{8} \mathrm{~B}$ neutrinos have typical energies around $6-7 \mathrm{MeV}$ while the ${ }^{7} \mathrm{Be}$ neutrinos have a lower energy of $0.862 \mathrm{MeV}$. For the ${ }^{7} \mathrm{Be}$ neutrinos the matter effects become nearly negligible and the $\nu_{e}$ survival probability is as given by vacuum oscillations. The angle $\theta$ here is the vacuum solar mixing angle $\theta_{\odot} \approx 35^{\circ}$. The oscillation factor $\sin ^{2}\left(1.27 \Delta m^{2} L / E\right)=1 / 2$ when averaged for fast oscillations. This means that it is possible to use the two flavour vacuum oscillation formula that after simplifications becomes:

$$
P\left(\nu_{e} \rightarrow \nu_{e}\right)=1-\frac{1}{2} \sin ^{2}\left(2 \theta_{\odot}\right) \approx 0.6
$$

for ${ }^{7}$ Be neutrinos. As the ${ }^{8} \mathrm{~B}$ neutrinos are more energetic, they undergo different physical oscillations. The ${ }^{8} \mathrm{~B}$ neutrinos are created as pure $\nu_{e}$ roughly at the centre of the Sun, where the electron density is $N_{e} \approx 6 \times 10^{25} / \mathrm{cm}^{3}$. The $\nu_{e}$ produced at the centre of the Sun are also pure $\nu_{2}$, as $\sin \left(2 \theta_{m}\right) \rightarrow 0$ at high electron density in equation (2.38). The neutrinos encounter appropriate conditions to undergo significant matter effects while travelling towards the exit of the Sun. The propagation of the neutrino is adiabatic since the density changes slowly in the Sun. This means that we can approximate to layers of the Sun in which the density is constant and study the neutrino propagation for this time and put together all the neutrino evolution as a time-ordered product. As the Hamiltonian is nearly diagonal and the eigenvalues of the Hamiltonian do not cross, the $\nu_{e}$ will leave the Sun still as mass eigenstates $\nu_{2}$, that can be expressed as:

$$
\nu_{2}=\nu_{e} \sin \theta_{\odot}+\nu_{x} \cos \theta_{\odot}
$$

The probability of observing $\nu_{e}$ on the Earth is the probability of observing a $\nu_{2}$ as a $\nu_{e}$, that is:

$$
P\left(\nu_{e} \rightarrow \nu_{e}\right)=\sin ^{2}\left(\theta_{\odot}\right)
$$

For values of $\theta_{\odot}<\pi / 4$, the survival probability in equation $(2.52)$ is less than $1 / 2$. In particular, the most accredited parameter values now are $\Delta m_{\odot}^{2}=(7.59 \pm 0.21) \times 10^{-5} \mathrm{eV}^{2}$ 
and $\tan ^{2} \theta_{\odot}^{N}=0.47_{-0.05}^{+0.06}[12]$.

The Borexino experiment [42] is a liquid scintillator detector that is currently taking data. Thanks to the high radiopurity it is able to detect low energy neutrinos, giving sensitivity to the ${ }^{7}$ Be neutrinos. The results obtained from the first data analysis report a number of $\nu_{e}$ from ${ }^{7}$ Be measured that is consistent with the expectation for the solar neutrino oscillation model. Further data from this experiment and the future SNO+ experiment [43], using a liquid scintillator Čerenkov detector, will help to confirm our understanding of solar neutrino oscillations. KamLAND is also planning to upgrade and achieve the necessary purity to observe ${ }^{7} \mathrm{Be}$ neutrinos.

\subsubsection{Atmospheric neutrinos}

Another line of evidence for neutrino oscillations comes from the atmospheric neutrinos. Neutrinos are produced in the atmosphere through processes like:

$$
\pi^{ \pm} \rightarrow \mu^{ \pm}+\nu_{\mu}\left(\bar{\nu}_{\mu}\right) \quad K^{ \pm} \rightarrow \mu^{ \pm}+\nu_{\mu}\left(\bar{\nu}_{\mu}\right) \quad \mu^{ \pm} \rightarrow e^{ \pm}+\bar{\nu}_{\mu}\left(\nu_{\mu}\right)+\nu_{e}\left(\bar{\nu}_{e}\right)
$$

The absolute flux of atmospheric neutrinos is known with an uncertainty of $20 \%$, while the ratio of the $\nu_{e}$ to the $\nu_{\mu}$ flux is known with a better accuracy of $5 \%$. The expected ratio is:

$$
\mathcal{R}=\frac{N_{\nu_{\mu}}+N_{\bar{\nu}_{\mu}}}{N_{\nu_{e}}+N_{\bar{\nu}_{e}}} \approx 2
$$

from $\pi$ decay, but varies with energy. Atmospheric neutrinos were first studied in experiments that were supposed to study proton decay. Experiments measured the double ratio:

$$
\mathcal{R}^{\prime}=\frac{\mathcal{R}_{\text {data }}}{\mathcal{R}_{\text {expected }}}
$$

The IMB experiment [44] (in 1985) and the Kamiokande experiment [45] (in 1988) reported a value for $\mathcal{R}^{\prime}$ significantly below unity. Both of the experiments used Cerenkov 


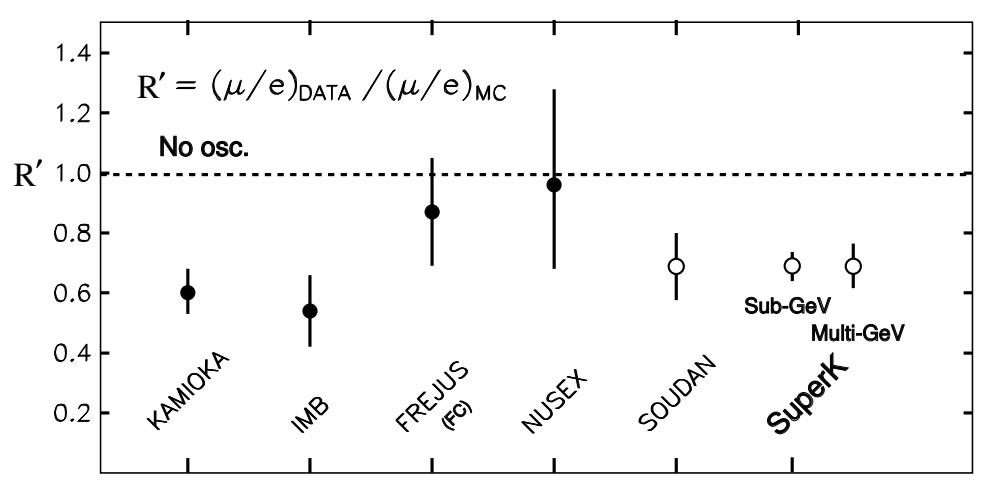

Figure 2.10: Atmospheric neutrino flux measurements. The quantity $\mathcal{R}^{\prime}$ is plotted from several experiments. Plot from [50].

detectors. On the other hand two other experiments, NUSEX [46] and Frejus [47], both iron calorimeters, measured a value of $\mathcal{R}^{\prime}$ consistent with unity. The discrepancy among the experiments was solved only when Soudan2 [48], another iron calorimeter experiment, measured a value of $\mathcal{R}^{\prime}$ consistent with the Cerenkov detector experiments. Following this, the Super-Kamiokande experiment measured $\mathcal{R}^{\prime}$ with great precision [49]. Figure 2.10 shows values obtained for $\mathcal{R}^{\prime}$ in various experiments. A value of $\mathcal{R}^{\prime}$ below unity suggests either that there is a deficit of $\nu_{\mu}, \bar{\nu}_{\mu}$ or that there is an appearance of $\nu_{e}, \bar{\nu}_{e}$.

Stronger evidence from atmospheric neutrino oscillations comes from the study of the neutrino flux as a function of the zenith angle $(\theta)$ in SK $[51,52,53]$. Downward-going neutrinos $(\cos \theta>0)$, produced in the atmosphere above the detector, only travel a short distance to the detector. On the other hand, upward-going particles $(\cos \theta<0)$ are produced in the atmosphere at the opposite side of the globe and have to cross the Earth to reach the detector. Figure 2.11 shows the distribution of events as a function of the zenith angle cosine for selected $\nu_{e}$ and $\nu_{\mu}$ events in two different energy ranges. While the selected $\nu_{e}$ events are consistent with the expectation for no oscillation, the $\nu_{\mu}$ events disagree with the no oscillation prediction and agree well with the oscillation fit. The low energy sample shows $\nu_{\mu}$ depletion for the entire spectrum of $\cos \theta$, while the high energy sample has a $\nu_{\mu}$ depletion only for long baseline (upward-going neutrinos). This is consistent with an energy dependent oscillation of $\nu_{\mu} \rightarrow \nu_{x}$, where $\nu_{x}$ is a non-electron neutrino.

In 2004 Super-Kamiokande released a new set of results [54] showing the oscillation 

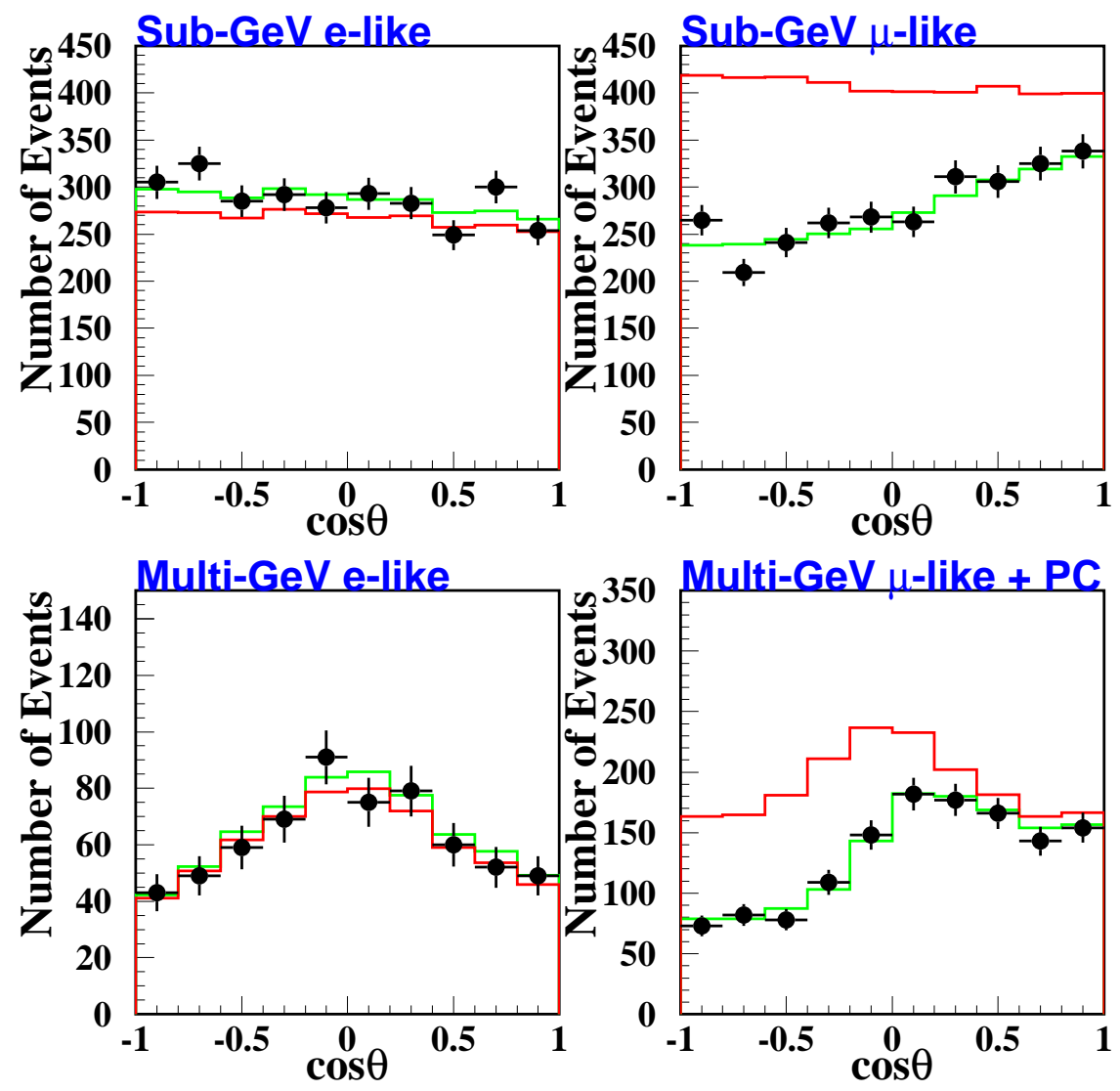

Figure 2.11: Distribution of the number of events observed as a function of the cosine of zenith angle $\cos \theta$ in the Super-Kamiokande experiment. The plots are for selected electron events on the left and selected muon events on the right. The top plots are for a low energy sample, while the bottom plots are for a higher energy sample. The red line is the distribution expected in the absence of oscillations, while the dotted histogram is for $\nu_{\mu} \rightarrow \nu_{\tau}$ oscillations. Figure taken from [53]. 


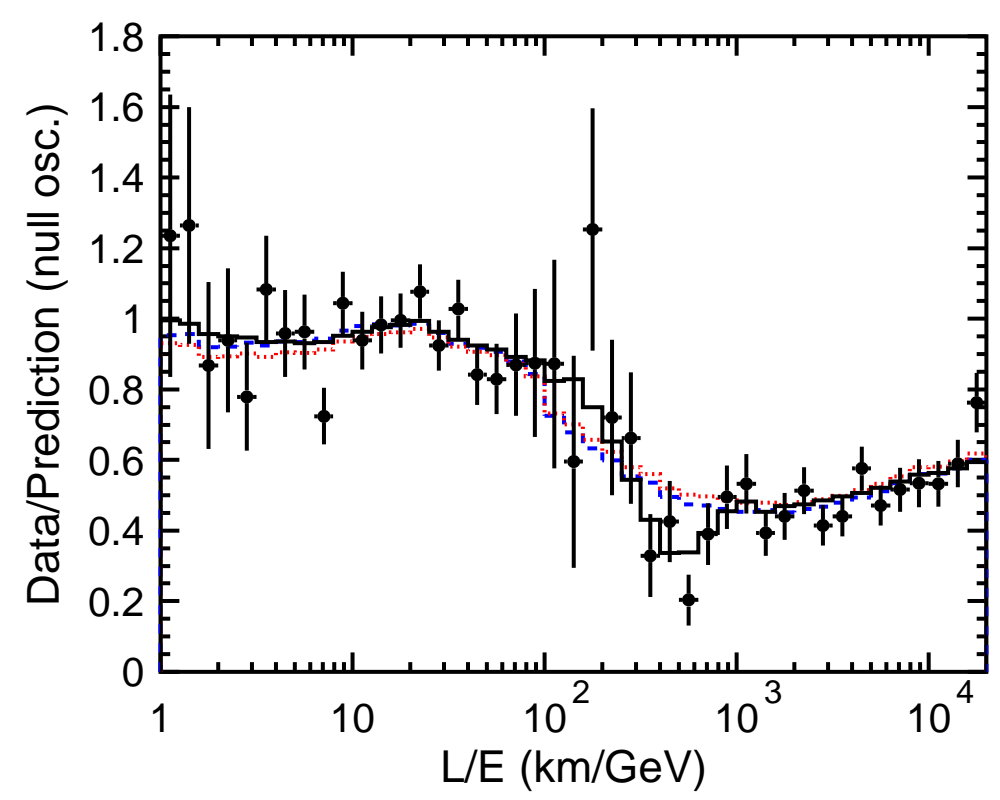

Figure 2.12: Ratio of the data to the unoscillated prediction as a function of $L / E$. The black solid line represents the best fit expectation for 2 flavour $\nu_{\mu} \rightarrow \nu_{\tau}$ oscillations. The error are statistical only. The dashed line represent the fit to neutrino decay, while the dotted line is the fit to neutrino decoherence: they represent alternative models to justify neutrino disappearance. Figure taken from [54].

pattern of data. The analysis of data was performed selecting a subset of events with high resolution on the neutrino energy $E$ and on the reconstructed distance travelled from production $L$ as determined from $\cos \theta$. Figure 2.12 shows the result of this analysis: the ratio between the selected data and the expectation in the case of no oscillation differs from unity as a function of $L / E$. The $L / E$ analysis provided the best limit on the mass splitting parameter at the time, but has been superseded by MINOS. SK still provides the best limits on the angle parameter in the atmospheric oscillations: $\sin ^{2}\left(2 \theta_{32}\right)>0.92$ at $90 \%$ C.L. [52]. In $2006 \mathrm{SK}$ released an analysis that disfavours no $\nu_{\tau}$ appearance by $2.4 \sigma$ in pure $\nu_{\mu} \rightarrow \nu_{\tau}$ oscillations [55]. SK has currently resumed operation and will be able to further contribute to solving the neutrino-puzzle.

The atmospheric neutrino oscillation parameters can also be studied with man-made beams. The $\mathrm{K} 2 \mathrm{~K}$ experiment produced a beam of $\nu_{\mu}$ from a proton beam colliding on a target and studied the $\nu_{\mu}$ spectrum after $250 \mathrm{~km}$ of propagation. K2K used a "near" detector, that is, a detector positioned soon after the neutrino production to obtain information 
about the flux and energy before neutrino oscillations. Super-Kamiokande was used as "far" detector to observe the neutrino beam after propagation. The near detector, a Cerenkov detector smaller but similar to SK, offers constraints on the far detector prediction and allows for cancellation of systematics. K2K measured oscillation parameters in agreement with the Super-Kamiokande atmospheric neutrino analysis and proved the feasibility of long baseline experiments [56].

The MINOS experiment, that will be discussed in the course of this thesis, has released several results on the atmospheric neutrino oscillation parameters. MINOS is a long baseline experiment: a beam of $\nu_{\mu}$ is produced, passes through the Near Detector and propagates for $735 \mathrm{~km}$. The $\nu_{\mu}$ interactions are detected at the Far Detector. MINOS results for the atmospheric mass splitting are the current most precise results: $\left|\Delta m_{23}^{2}\right|=2.43 \pm 0.13 \times$ $10^{-3} \mathrm{eV}^{2}$ at $68 \%$ C.L. [57]. Updated results have just been presented in June 2010 at a conference [58]: $\left|\Delta m_{23}^{2}\right|=2.35_{-0.08}^{+0.11} \times 10^{-3} \mathrm{eV}^{2}$ at $68 \%$ C.L. Figure 2.13 shows the atmospheric oscillation parameters from MINOS, in comparison to previous results from SK and K2K.

The OPERA experiment is another long baseline neutrino oscillation search experiment, with quite similar characteristics to MINOS (a beam of $\nu_{\mu}$ propagates for a baseline of 730 $\mathrm{km})$. The major difference is that the main analysis goal in OPERA is direct detection of $\nu_{\mu} \rightarrow \nu_{\tau}$ transitions. To achieve this goal OPERA uses photographic emulsions to detect the distinctive "kick" left by the $\tau$ when it decays into $\mu \nu_{\mu} \nu_{\tau}$. Note that OPERA (and MINOS) started construction when the atmospheric $\Delta m^{2}$ was believed to be a factor of 3 higher than at present. MINOS has been affected by this but in smaller proportion than OPERA. In MINOS it was chosen to run in the lowest beam energy configuration to match the $L / E$ required by the analysis. This has also happened for OPERA, but the limitation comes from the fact that the threshold energy required for a $\nu_{\tau}$ to produce a $\tau$ in a charged current interaction is $\approx 3.5 \mathrm{GeV}$, putting a limit on the lower beam energy to see direct $\tau$ appearance. The OPERA collaboration has publicly announced their first $\nu_{\tau}$ candidate event [59]: the background expected is $0.045 \pm 0.023$. This corresponds to a $4.5 \%$ probability of observing a fluctuation of the background to fake the signal event: the significance of 


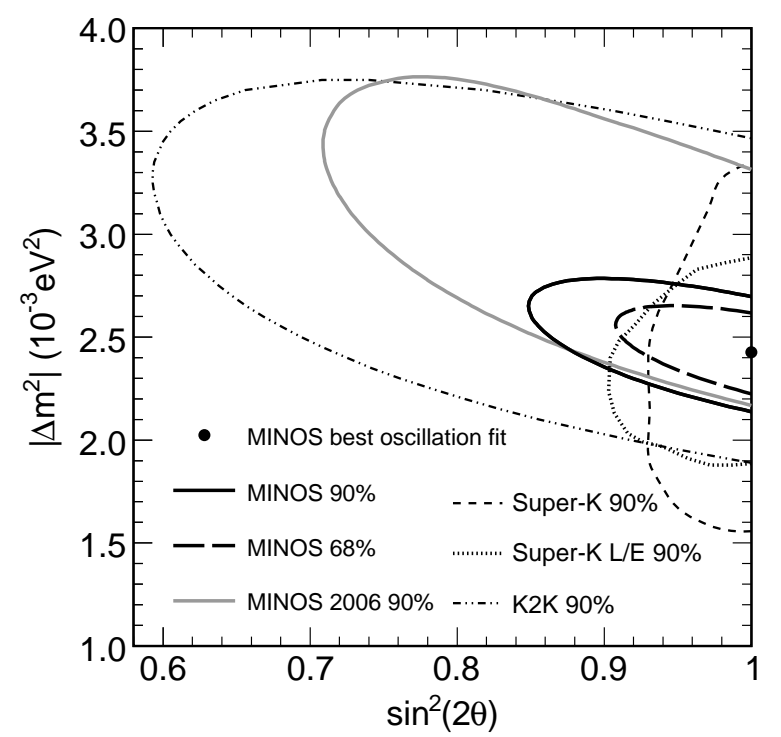

Figure 2.13: The plot shows the MINOS contour for the atmospheric oscillation parameters in black at $90 \%$ C.L. and in hatched black for the $68 \%$ C.L. The grey contour represent an old MINOS analysis contour, with limited statistics. The finer line contours represent results from other experiements. The biggest hatched contour represents the $\mathrm{K} 2 \mathrm{~K}$ experiment results at $90 \%$ C.L. The hatched line is the result of the SK $L / E$ analysis and the other finely hatched line is the contour for the SK results at $90 \%$ C.L. Figure from [57]. 


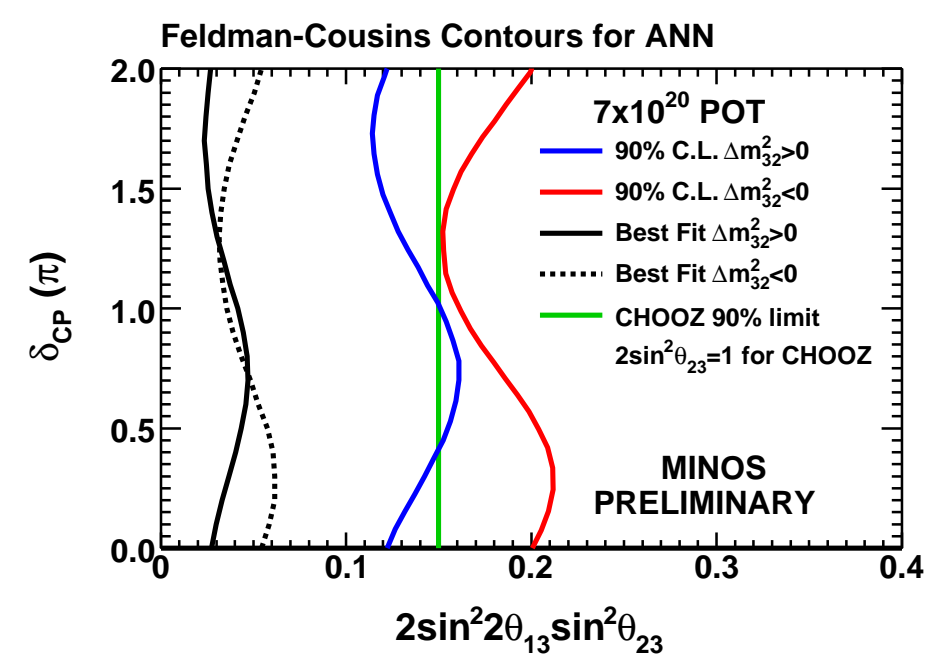

Figure 2.14: The figure shows the allowed region for $2 \sin ^{2}\left(\theta_{23}\right) \sin ^{2}\left(2 \theta_{13}\right)$ as a function of the CP violating phase $\delta$ as obtained by MINOS at $90 \%$ C.L. in the case of normal hierarchy in blue and in the case of inverted hierarchy in red. The best fit values in the two cases are given by the solid black and the hatched black lines. Superimposed is the limit obtained by the CHOOZ experiment at $\sin ^{2}\left(\theta_{23}\right)=1$. The plot is taken from [64].

the observed event is $2.01 \sigma$ [59]. Other future experiments like $\mathrm{NO} \nu \mathrm{A}[60]$ and $\mathrm{T} 2 \mathrm{~K}$ [61] can put even tighter constraints on the atmospheric neutrino oscillation parameters.

\subsection{3 $\theta_{13}$ and $\delta$}

In the case of CPT being conserved, reactor experiments can be sensitive to $\theta_{13}$ at the atmospheric mass splitting by looking at the $\bar{\nu}_{e}$ survival probability, as in equation (2.26). Experiments like Bugey and PaloVerde were placed at a $L / E$ close to the atmospheric one, but they were not exactly in the optimal conditions. The CHOOZ experiment, a liquid scintillator detector, was the best located in terms of baseline from a power station and it is currently the experiment that establishes the best measurement on $\theta_{13}: \sin ^{2}\left(2 \theta_{13}\right)<$ 0.13 at $\Delta m_{23}^{2}=2.7 \times 10^{-3} \mathrm{eV}^{2}$ [29]. Recently MINOS has also performed an appearance analysis for $\nu_{\mu} \rightarrow \nu_{e}$, giving limits on $\theta_{13}[62,63]$. Figure 2.14 shows the region allowed for $2 \sin ^{2}\left(\theta_{23}\right) \sin ^{2}\left(2 \theta_{13}\right)$ versus the CP violating phase $\delta$ obtained by MINOS at $90 \%$ C.L. in case of normal or inverted hierarchy. The CHOOZ limit for $\sin ^{2}\left(\theta_{23}\right)=1$ is superimposed.

Future experiments are being built now to provide more accurate information on $\theta_{13}$ 
and the $\mathrm{CP}$ (violating) phase $\delta$. In particular there are two classes of experiments. The first type are reactor experiments: Double Chooz [65], Daya Bay [66] and Reno [67], whose goal is to measure or constrain $\theta_{13}$ through the survival probability for $\bar{\nu}_{e}$. The second class of experiments are off-axis beam long baseline experiments: T2K and No $\nu \mathrm{A}$. By moving the far detector to be slightly away from the centre of the beam, neutrino energies are less dependent on the parent pion momentum, limiting the impact of the uncertainty in the secondary hadron production. The T2K experiment, which will employ SK as far detector, will have a baseline of $295 \mathrm{~km}$ and mean neutrino energy of $600 \mathrm{MeV}$. The experiment will have two near detectors: one on-axis and one off-axis. The goal is to extract flux information and background information (mainly $\pi^{0}$ produced in NC interactions that can fake signal $\left.\nu_{e}\right)$. The two near detectors are significantly different in characteristics with SK; however the off-axis near detector will have a water target to extract $\pi^{0}$ production cross section in water. Apart from improving the atmospheric neutrino oscillation parameters $\sin ^{2} \theta_{23}$ and $\left|\Delta m_{32}^{2}\right|$ by looking at $\nu_{\mu} \rightarrow \nu_{\mu}$ processes, T2K will primarily look at the sub-dominant oscillations $\nu_{\mu} \rightarrow \nu_{e}$.

The NO $\nu \mathrm{A}$ experiment is designed to pursue the same physics program as $\mathrm{T} 2 \mathrm{~K}$, but it is also more sensitive to matter effects, given the longer baseline of $810 \mathrm{~km}$. NO $\nu \mathrm{A}$ will use two functionally identical tracking liquid scintillator calorimeters as near and far detector. $\mathrm{NO} \nu \mathrm{A}$ will look at $\nu_{\mu}$ disappearance and at $\nu_{\mu} \rightarrow \nu_{e}$ results. Following the arguments in [68], experiments like $\mathrm{T} 2 \mathrm{~K}$ and $\mathrm{NO} \nu \mathrm{A}$ will experience two competing effects in the probability $P\left(\nu_{\mu} \rightarrow \nu_{e}\right)$ : the presence of the CP violating phase (arising from the threeflavour oscillation probability) and the matter effects that can mimic one another. Some information from external experiments needs to be introduced to resolve the ambiguity between the apparent CP phase measured and matter effects. Far future experiments, like DUSEL or T2KK (still at the design stage), will have an even longer baseline in order to reach the second maximum of the oscillation probability where the effect of $\delta$ and matter effects can be disentangled. 


\subsubsection{Sterile neutrino hints}

The simplest interpretation of the results coming from the Liquid Scintillator Neutrino Detector (LSND) experiment requires the existence of a fourth neutrino mass state. The LSND experiment has studied $\bar{\nu}_{\mu} \rightarrow \bar{\nu}_{e}$ transitions from $\mu^{+}$decay at rest [69] in 1996. Two years later the same collaboration has also released results with lower statistics for $\nu_{\mu} \rightarrow \nu_{e}$ transitions from $\pi^{+}$decaying in flight [70]. For both the analyses, the results showed an excess of $\nu_{e} / \bar{\nu}_{e}$ compatible with neutrino oscillations at a mass splitting greater than $0.4 \mathrm{eV}^{2}$. This result is incompatible with the solar and atmospheric mass splittings and requires the introduction of at least one sterile neutrino to allow a much higher splitting. Other experiments had earlier looked at the parameter space compatible with LSND. In particular the KARMEN2 experiment was the most similar in characteristic with LSND. They studied $\bar{\nu}_{\mu} \rightarrow \bar{\nu}_{e}$ transitions from muons at rest, with results compatible with no evidence of oscillations at mass splitting lower than $7 \mathrm{eV}^{2}[71]$.

The MiniBooNE experiment has the ultimate goal to confirm or exclude LSND results on sterile neutrinos at $\mathcal{O}\left(1 \mathrm{eV}^{2}\right)$. A beam of $\nu_{\mu}$ or $\bar{\nu}_{\mu}$ is allowed to propagate over a short-baseline to a Čerenkov detector. MiniBooNE is undertaking a $\nu_{\mu} \rightarrow \nu_{e}$ or $\bar{\nu}_{\mu} \rightarrow \bar{\nu}_{e}$ appearance search. The experiment has released results for $\nu_{e}$ appearance, observing no evidence of an excess of $\nu_{e}$ events above $475 \mathrm{MeV}$ [72]. This, in case of no CPT and CP violation, rules out the LSND results. Nevertheless, an excess of $\nu_{e}$ events has been observed at lower energies, but is not relevant for the LSND mass splitting analysis. Figure 2.15 shows the exclusion oscillation parameters contour for the MiniBooNE experiment (running with $\nu_{\mu}$ ) in comparison with the LSND results. The MiniBooNE collaboration has started running with a $\bar{\nu}_{\mu}$ beam, to be more consistent with the LSND conditions. Results with the first dataset have been released [73]. No significant excess of events has been observed, both at low energy (200-475 MeV) and at high energy (475-1250 MeV). In June 2010, updated antineutrino results have been presented at a conference [74] and show data excess both at low energy (like in the neutrino case) and at high energy (in the LSND $L / E$ region). 


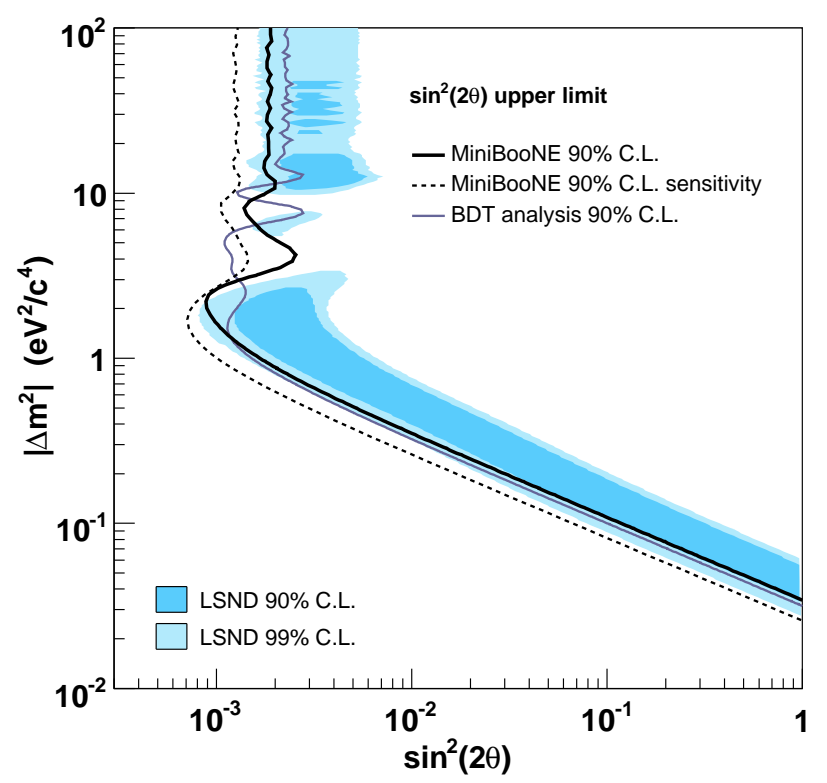

Figure 2.15: The figure shows the oscillation parameters excluded by the MiniBooNE experiment from the neutrino running: the solid line is the $90 \%$ C.L. and the dotted line represents the predicted sensitivity for the same measurement. In comparison the LSND allowed region is shown in blue at 90\% C.L. Figure taken from [72].

The oscillation fit has been performed and, considering only the events above $475 \mathrm{MeV}$, the 90\% C.L. contour overlaps with the LSND 90\% C.L. contour.

The introduction of 1 sterile neutrino is only marginally compatible with the data, both in the $3+1$ scenario or in the even more disfavoured $2+2$ scenario. With two additional sterile neutrinos $(3+2$ model) MiniBooNE neutrino results and LSND antineutrino results are still compatible thanks to the $\mathrm{CP}$ violation possible in this scheme [31].

The SK collaboration has looked for $\nu_{\mu} \rightarrow \nu_{s}$ transitions in atmospheric $\nu_{\mu}$ oscillations in $[75,76]$. The SK group has analysed two different effects that could be sensitive to the existence of sterile neutrinos: the consistency of the rate of neutral current interactions (which are only sensitive to mixing to sterile neutrinos) and matter-induced effects (which would leave the probability $P\left(\nu_{\mu} \rightarrow \nu_{\tau}\right)$ unchanged as $\nu_{\mu}$ and $\nu_{\tau}$ interact in the same way in matter, but would create an effective potential in the oscillation probability $P\left(\nu_{\mu} \rightarrow \nu_{s}\right)$ as sterile neutrinos do not interact in matter). Pure $\nu_{\mu} \rightarrow \nu_{s}$ were disfavoured by more than $5 \sigma$ compared to pure $\nu_{\mu} \rightarrow \nu_{\tau}$ oscillations [76]. Mixing with sterile neutrinos is still 
possible as a sub-dominant process, like in $\nu_{\mu} \rightarrow\left(\cos \xi \nu_{\tau}+\sin \xi \nu_{s}\right)$. The maximum fraction allowed for mixing with sterile neutrinos is $\sin ^{2} \xi<0.19$ at $90 \%$ C.L [77].

MINOS has also studied limits on sterile neutrinos $[78,30]$. The fraction of $\nu_{\mu}$ which change flavour which could be converted into sterile neutrino is less than $52 \%$ at $90 \%$ C.L. In June 2010 MINOS updated results were presented in a conference [58]: the fraction of neutrinos that could have oscillated into sterile neutrinos is less than $0.22(0.40)$ if $\theta_{13}=$ $0^{\circ}\left(11.5^{\circ}\right)$

\subsection{Conclusions: overview of global oscillation results}

Figure 2.16 shows the present phase space allowed for the neutrinos mass splitting $\Delta m^{2}$ and angles, expressed in the form of $\tan ^{2} \theta$ for neutrino oscillation experiments.

The plot is rich in information and covers the three relevant mass splittings that have been considered so far.

\subsubsection{Solar $\Delta m^{2}$ mass splitting}

- As already mentioned, if $\nu_{e}$ oscillations were only happening in vacuum, the plot would be symmetric around $\tan ^{2}\left(\theta_{12}\right)=1$. Neutrino oscillations in matter break the symmetry in the allowed parameters, as visible from figure 2.16.

- To explain matter oscillations, it is required to consider that the experiments considered so far are sensitive to different solar neutrino energy ranges. The radiochemical experiments are partially sensitive to the ${ }^{7} \mathrm{Be}$ and $p p$ neutrinos, whereas SK and SNO are sensitive only to ${ }^{8} \mathrm{~B}$ neutrinos. Matter effects enhance the disappearance of ${ }^{8} \mathrm{~B}$ neutrinos.

- Historically, there has been an ambiguity of solutions, that have been ruled out by adding information when new experiments came along. The most common solutions taken into account for the solar mass splitting were: 


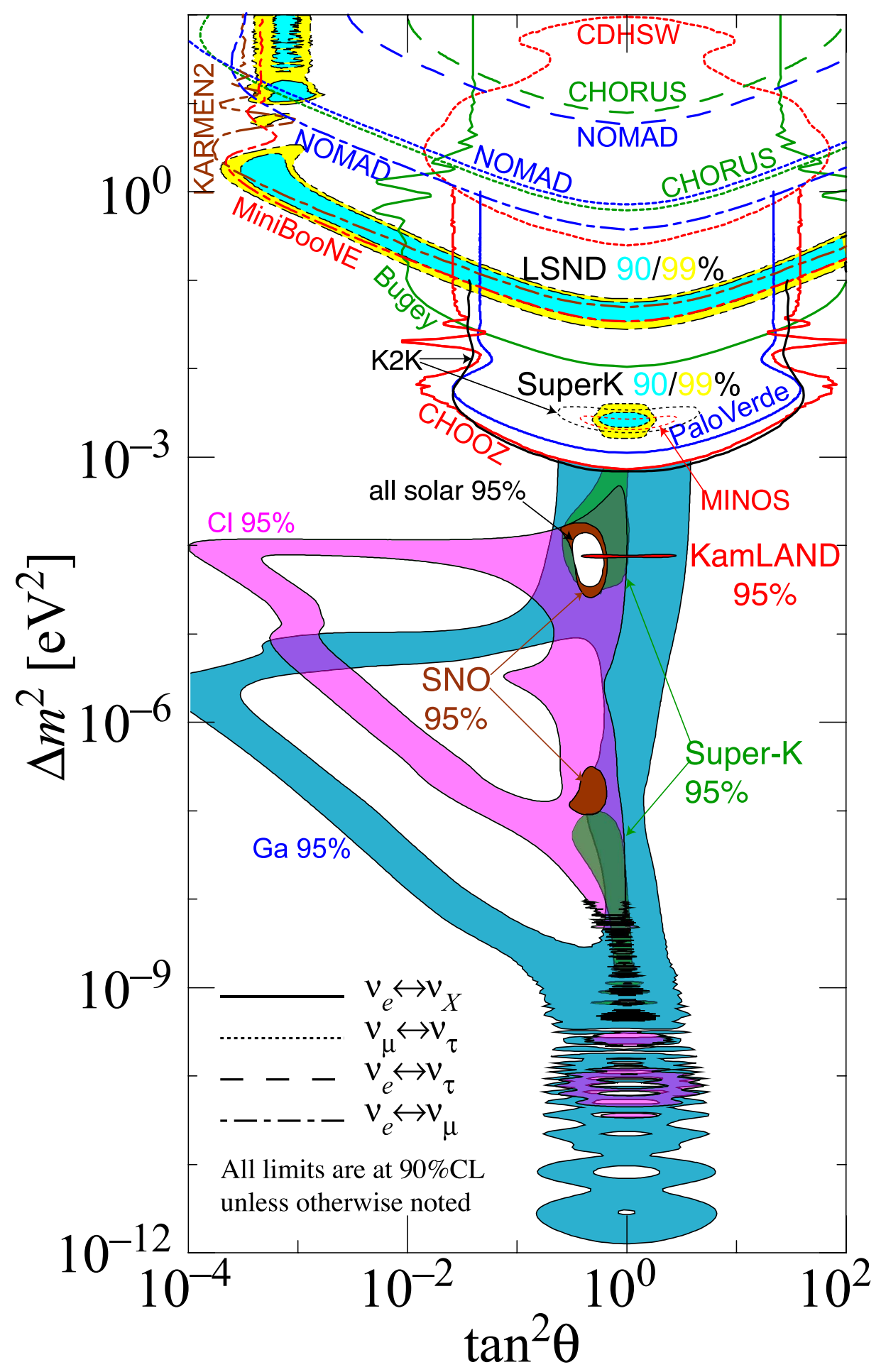

Figure 2.16: A summary of the constraints on neutrino oscillation parameters using all data to date. Filled, coloured areas represent allowed regions. Lines not surrounding filled regions are exclusion limits: when these lines do not close the region above the line is excluded, otherwise the region within the line is excluded. Figure taken from [12]. 
- Large mixing angle (LMA): $\Delta m^{2} \approx 5.0 \times 10^{-5} \mathrm{eV}^{2}, \tan ^{2} \theta \approx 0.42$,

- Low mass $(\mathrm{LOW}): \Delta m^{2} \approx 7.9 \times 10^{-8} \mathrm{eV}^{2}, \tan ^{2} \theta \approx 0.61$

- Vacuum (VAC): $\Delta m^{2} \approx 4.6 \times 10^{-10} \mathrm{eV}^{2}, \tan ^{2} \theta \approx 1.8$

- Small mixing angle (SMA): $\Delta m^{2} \approx 5.0 \times 10^{-6} \mathrm{eV}^{2}, \tan ^{2} \theta \approx 1.5 \times 10^{-3}$.

- The SMA solution was once favoured but after SNO it has been excluded at $3 \sigma$. The latest KamLAND results (assuming CPT invariance) are in favour of the LMA solution.

\subsubsection{Atmospheric $\Delta m^{2}$ mass splitting}

The atmospheric mass splitting is two orders of magnitude larger than the solar mass splitting. Information about the atmospheric mass splitting can be found via $\nu_{\mu} \rightarrow \nu_{\mu}$ channels (either looking at atmospheric neutrinos or beam long baseline experiments) or through the $\bar{\nu}_{e} \rightarrow \bar{\nu}_{e}$ channel at reactor experiments. Experiments are generally in agreement on the measurement of this mass splitting. In this case of atmospheric neutrino and long baseline measurements, the parameters for neutrino oscillations are given by $\left|\Delta m_{32}^{2}\right|$ and $\theta_{23}$. Experiments studying $\bar{\nu}_{e} \rightarrow \bar{\nu}_{e}$ oscillations are described by the parameters $\Delta m_{e e}^{2}$ (the atmospheric effective mass splitting) and $\theta_{13}$.

\subsubsection{Sterile $\Delta m^{2}$ mass splitting}

Results from the LSND experiment have required the introduction of a much higher mass splitting, associated to a sterile neutrino. The MiniBooNE experiment has partially ruled out the higher mass splitting, but a possibility of explaining the LSND results remains open allowing $\mathrm{CP}$ to be violated and play a role in the difference between neutrinos and antineutrino oscillations. This thesis will study the high mass splitting up to $\mathcal{O}\left(1 \mathrm{eV}^{2}\right)$, helping to interpret the LSND results. 


\section{Chapter 3}

\section{The MINOS experiment}

This chapter describes the MINOS experiment. Section 3.1 introduces the physics results the experiment has achieved and the prospects for potential improvements. Section 3.2 describes the NuMI beamline: a more detailed treatment can be found in $[79,80]$. The detector characteristics are presented in section 3.3. An exhaustive description of the MINOS detectors is in $[81,80]$. Sections 3.4 and 3.5 are dedicated to the simulation software used for the MC and the event reconstruction software which is used in both data and MC. The description of the dataset analysed in this thesis is given in section 3.6.

\subsection{The physics capabilities of MINOS}

MINOS has been optimised to precisely measure the atmospheric oscillation parameters $\sin ^{2}\left(2 \theta_{23}\right)$ and $\left|\Delta m_{32}^{2}\right|$ : the measurement is obtained through a $\nu_{\mu}$ two-flavour disappearance analysis for charged current interactions, where the charged current data spectrum is compared to the MC spectrum oscillated according to the probability in equation (2.24). On the left, figure 3.1 shows the Far Detector spectrum for selected charged current events as in [57]. Data, the expectation in the absence of oscillations and the best fit to a twoflavour neutrino oscillation model are shown. The plot on the right shows the ratio of the data and the best fit MC spectrum to the unoscillated prediction as well as other alternative 

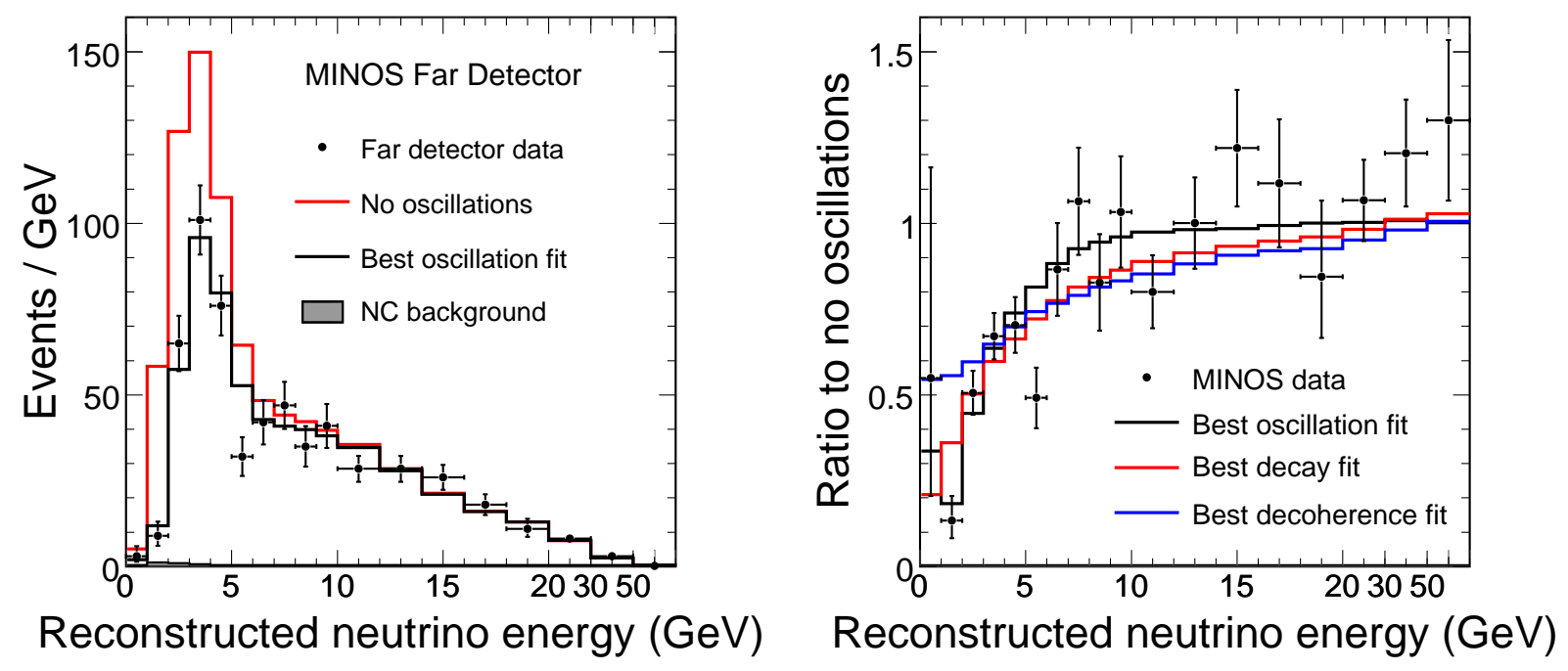

Figure 3.1: The plot on the left shows the MINOS Far Detector charged current spectrum for data in black dots, as expected from MC in absence of oscillations in red, and the best fit to a two flavour oscillation in black. The plot on the right shows the ratio of the data to the unoscillated prediction in black dots, the ratio of the best fit oscillated spectrum to the unoscillated prediction in black. The results for alternative models which justify $\nu_{\mu}$ disappearance are also superimposed. The plots are taken from [57].

models which may justify neutrino disappearance. The energy dependent depletion of the spectrum is clearly visible from the plot. The results obtained for the two-flavour oscillation model $\operatorname{are~} \sin ^{2}\left(2 \theta_{23}\right)>0.90$ at $90 \%$ C.L. and $\left|\Delta m_{32}^{2}\right|=(2.43 \pm 0.13) \times 10^{-3} \mathrm{eV}^{2}$ at $68 \%$ C.L.: the measurement of the atmospheric mass splitting by MINOS is the most precise in the world (superseded by updated results in June 2010 [58]). The 90\% C.L. contour in the two oscillation parameters has been shown in figure 2.13.

As the MINOS detectors are magnetised, it is possible to discriminate between $\mu^{-}$ coming from $\nu_{\mu}$ and $\mu^{+}$coming from $\bar{\nu}_{\mu} . \bar{\nu}_{\mu}$ are a $7 \%$ background which is produced in the beam in the normal MINOS running. A first analysis of antineutrino oscillations, with low statistics, has been presented in [82]. 42 events were measured at the Far Detector in contrast to an expectation of $58.3 \pm 7.6$ (stat.) \pm 3.6 (syst.) events in the case $\bar{\nu}_{\mu}$ oscillate with the same parameteters as $\nu_{\mu}$. Given the anomaly of the results, a full run (Run IV) has been dedicated to $\bar{\nu}_{\mu}$ oscillation measurement. In June 2010 conference results were 
released on the antineutrino oscillation parameters [58]: $\left|\overline{\Delta m_{23}^{2}}\right|=3.36_{-0.40}^{+0.45} \times 10^{-3} \mathrm{eV}^{2}$ and $\sin ^{2} 2 \bar{\theta}=0.86 \pm 0.11$. Increased statistics are necessary to understand if antineutrino oscillation parameters are different from the neutrino ones.

The other contribution that MINOS can give to the neutrino studies is a limit on $\theta_{13}$ obtained from $\nu_{e}$ appearance at the Far Detector through the subdominant oscillations $\nu_{\mu} \rightarrow \nu_{e}$. The limit set by MINOS on $\theta_{13}$ have been shown in section 2.4.3.

MINOS can study sterile neutrino limits by analysing the neutral current selected spectrum, which is unchanged by oscillations among active neutrinos. Results have been published in [30], with the fraction of $\nu_{\mu}$ which can convert into a sterile neutrinos limited to be $50 \%$ at $90 \%$ C.L. This thesis analyses sterile neutrino mixing with $\Delta m_{43}^{2} \approx \mathcal{O}\left(1 \mathrm{eV}^{2}\right)$, according to the model explained in section 2.3.4, with increased statistics compared to the published article (from $3.18 \times 10^{20}$ to $7 \times 10^{20}$ protons on target) and improved techniques. Figure 3.2 shows the 90\% C.L. contours from [30] obtained for angle parameters in the model considered.

Apart from the beam neutrino oscillations, MINOS has studied neutrino oscillations [83] with neutrinos coming from the atmosphere and cosmic ray fluxes $[84,85]$ exploiting the underground location and the magnetisation of the detectors. The Near Detector can be used for neutrino cross section measurements [86].

\subsection{The NuMI neutrino beam}

The Neutrinos at the Main Injector (NuMI) beamline is located at Fermilab and produces primarily $\nu_{\mu}$ from a primary proton beam colliding on a target. Protons of $120 \mathrm{GeV} / c$ momentum are extracted from the Main Injector in $10 \mu$ s spills. As the Main Injector accepts batches of protons to accelerate from the Booster, neutrinos will show a time distribution in batches as well. The typical intensity of the proton beam is $2.1 \times 10^{13}$ protons on target with a cycle time of $2.2 \mathrm{~s}$. Efforts have been dedicated to increase the intensity to $4 \times 10^{13}$ protons on target. 


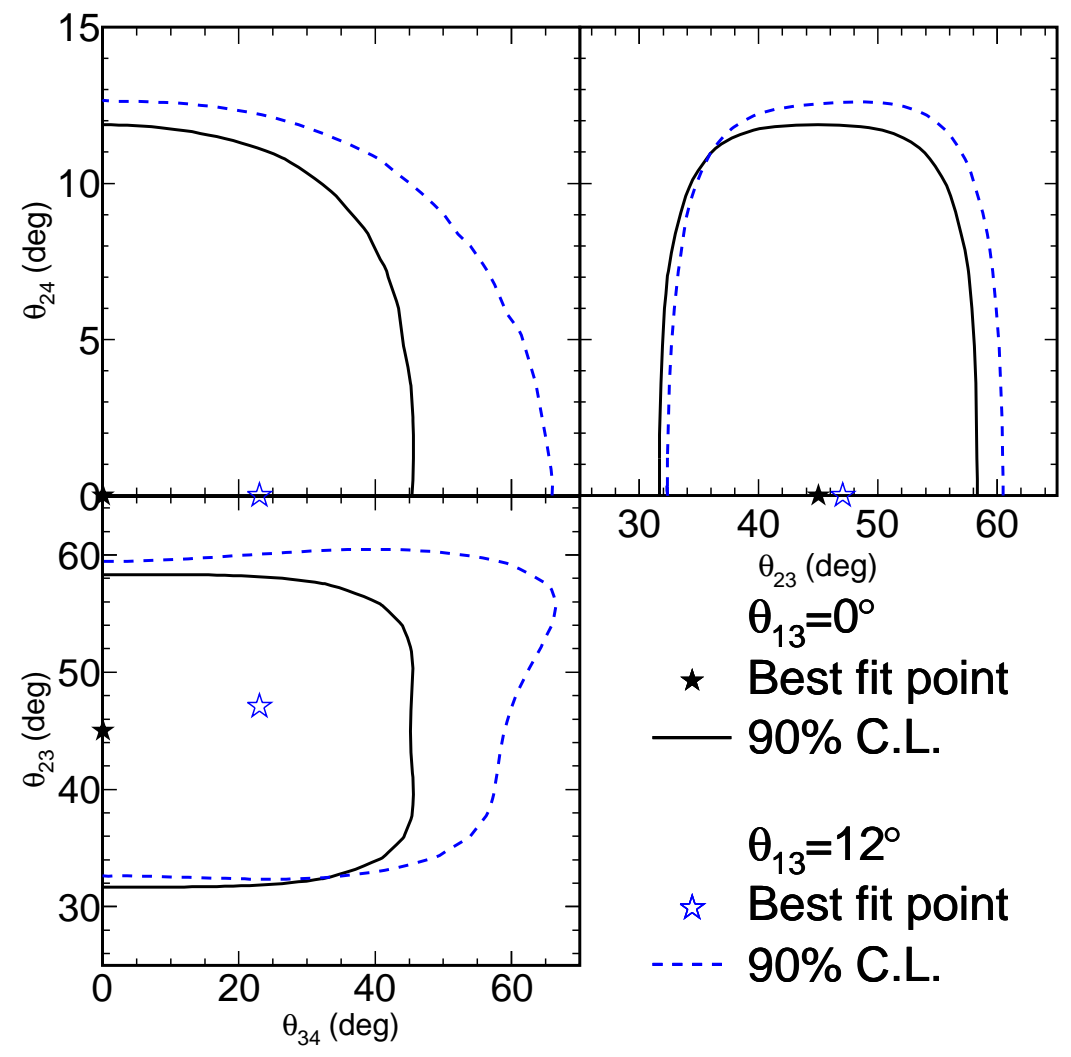

Figure 3.2: The 90\% C.L. contours obtained in [30] for the angle parameters in the model fitted in this thesis. 


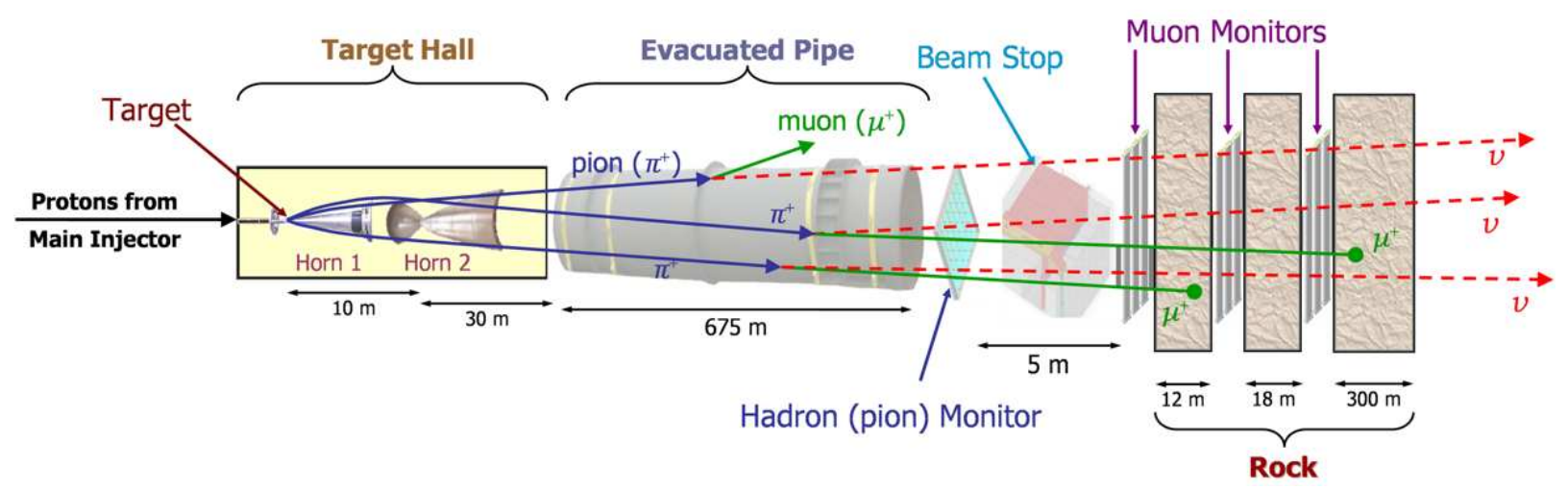

Figure 3.3: The components of the NuMI beamline. $120 \mathrm{GeV}$ protons from the Main Injector collide on a graphite target. Secondary mesons are focused and produce neutrinos from their decay.

Figure 3.3 shows a schematic diagram of the beam line. The primary proton beam hits a graphite target with dimensions $6.4 \times 15 \times 940 \mathrm{~mm}^{3}$ that is segmented longitudinally into 47 fins (with a total thickness of 1.9 interaction length). The target needs to be water cooled at the top and bottom of each fin. Secondary particles from the target, in particular pions and kaons or tertiary particles deriving from those, are magnetically selected in charge and momentum using the horns. By adjusting the current in the horns and the relative position of the target in respect to the first horn, it is possible to change the range of momenta of the selected mesons. The magnetic field produced by the horns can be reversed to produce a $\bar{\nu}_{\mu}$ beam. The mesons focused by the horns enter the decay pipe, which is $675 \mathrm{~m}$ long and evacuated to reduce particle absorption. At the end of 2007, it was necessary to fill the decay pipe with helium at 0.9 atm as the upstream end of the decay pipe was found to be corroded by the beam activity and there were concerns of implosion. Neutrinos are produced from decay of pions and kaons, as shown in equations (2.53). At the end of the decay pipe there is a set of beam absorbers; the final part consists of $300 \mathrm{~m}$ of rock which is used to stop muons and any remaining hadrons. Ionisation chambers are used to monitor the secondary and tertiary products (hadrons and muons) of the proton-target interaction and there are ongoing studies to independently extract the neutrino flux from those monitors [87]. The neutrino beam needs to be inclined downstream by $3.3^{\circ}$ to point at the Far Detector. 

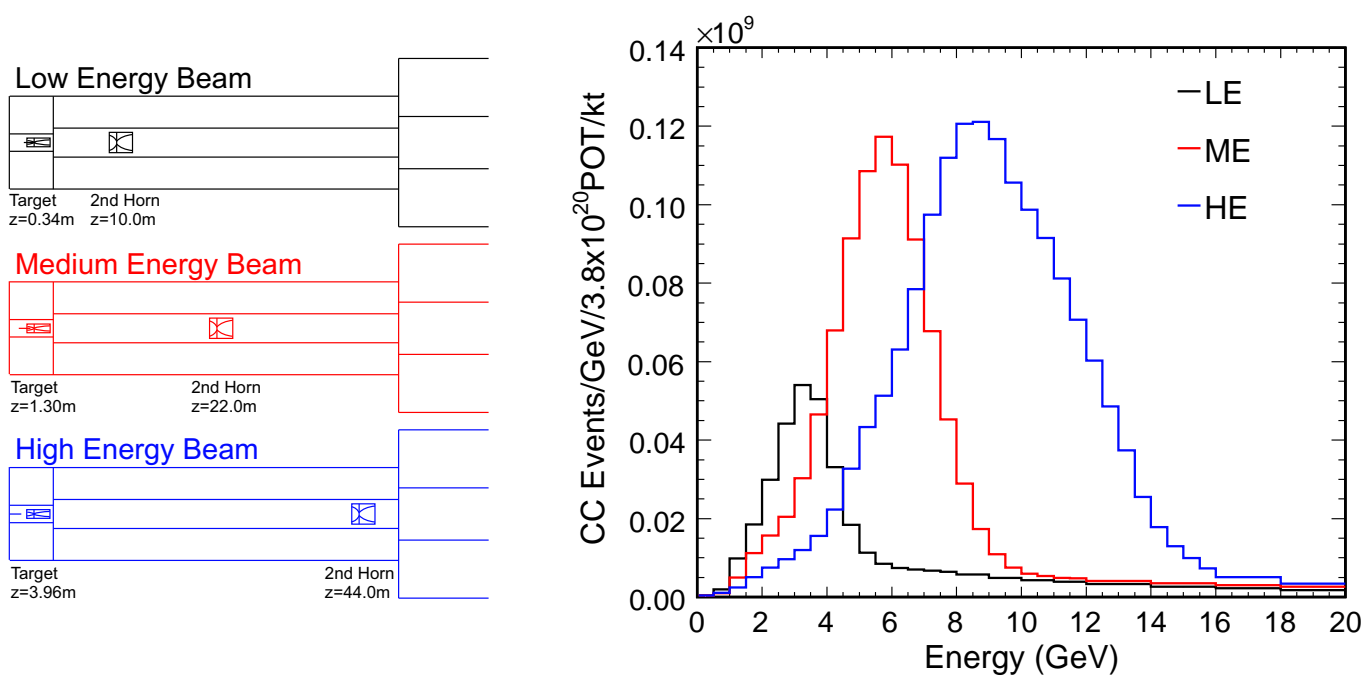

Figure 3.4: Three possible configurations of the NuMI beam (low, medium and high energy). The relative target and focusing horn positions are shown on the left and the corresponding $\nu_{\mu}$ energy spectra on the right. The beam in normal operation is in the lower energy configuration.

The adaptability of the NuMI beamline to change the momenta of the meson focused allows multiple neutrino energy configurations. The energy of the neutrinos $E_{\nu}$ is related to the energy $E$ of the mesons selected using the two-body decay kinematics:

$$
E_{\nu_{\mu}}=\frac{0.43 E}{1+E^{2} \theta^{2} / m_{\pi / \mathrm{K}}^{2}}
$$

where $\theta$ represents the angle in the laboratory frame between the $\nu$ and the parent meson direction. Figure 3.4 shows the configuration for the horns in respect to the target position to obtain different neutrino energy spectra. To properly match the atmospheric $L / E$ required by the oscillation analysis, MINOS uses the lowest energy spectrum configurable, which is technically referred to as LE010z185i, as the target is displaced $10 \mathrm{~cm}$ from its nominal position inside the first horn and the horn current is $185 \mathrm{kA}$. During the data taking, the target has been moved $1 \mathrm{~cm}$ further away from the second horn. The MINOS Near Detector is located nearly $1 \mathrm{~km}$ downstream from the target, while the Far Detector is $735 \mathrm{~km}$ away from the target. The two solid angles subtended by the beam at the Near Detector and at the Far Detector are somewhat different, as only neutrinos that have been emitted as a 
point-like source approximation can arrive at the Far Detector. On the contrary, the Near Detector accepts a broader range of angles. This difference between the Near Detector and Far Detector is small and in first approximation it is possible to consider the transfer matrix diagonal. The precise treatment of the transfer matrix is available in [80].

\subsection{The MINOS detectors}

The MINOS detectors are designed to be functionally similar in order to cancel common systematics. Two detectors (the Near Detector and the Far Detector) are exposed to the NuMI neutrino beamline. Figure 3.5 shows the pictures of the Near Detector (left) and Far Detector (right). A third detector, called Calibration Detector, was a smaller detector prototype for test-beam studies with very similar construction to the main detectors. The detectors are scintillator/steel sampling calorimeters. The active scintillating medium (extruded polystyrene with PPO and POPOP as scintillating component) is segmented into strips, which are $4.1 \mathrm{~cm}$ wide and $1.0 \mathrm{~cm}$ thick. Figure 3.6 shows a sketch of a scintillating strip in MINOS. The strips are coated with a layer of reflective co-extruded titanium-dioxide $\left(\mathrm{TiO}_{2}\right)$. A wavelength-shifting fibre is embedded in the scintillating strip and carries the signal to the photomultiplier tubes; the wavelength-shifting fibres shift the scintillator light from blue $(\lambda \approx 420 \mathrm{~nm})$ to green $(\lambda \approx 470 \mathrm{~nm})$. The scintillating strips in consecutive planes are oriented $90^{\circ}$ from each other to allow $3 \mathrm{D}$ tracking. The strips are oriented $\pm 45^{\circ}$ from the vertical and they define the $u$ and $v$ directions, in contrast to the horizontal direction $x$ and vertical direction $y$ in the MINOS coordinate system. The beam direction is referred to as $z$. The steel planes are $2.54 \mathrm{~cm}$ thick; the distance between two successive steel+scintillator planes is $5.94 \mathrm{~cm}$, leaving empty space for air. Both the main detectors have a toroidal magnetic field. Figure 3.7 shows a not-to-scale cartoon which describes the geometry of the sampling calorimeters. The photomultipliers are operated at $800 \mathrm{~V}$, with typical gain $\approx 10^{6}$ photo-electrons.

A light injection system is used at all detectors to monitor the stability of of the pho- 

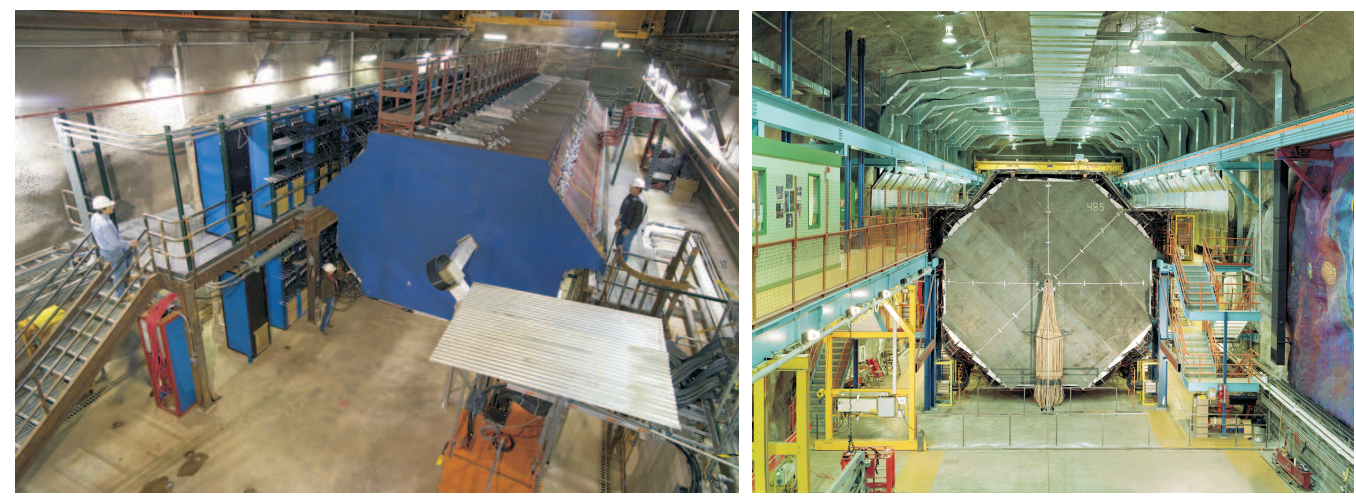

Figure 3.5: The MINOS near (left) and far (right) detectors.

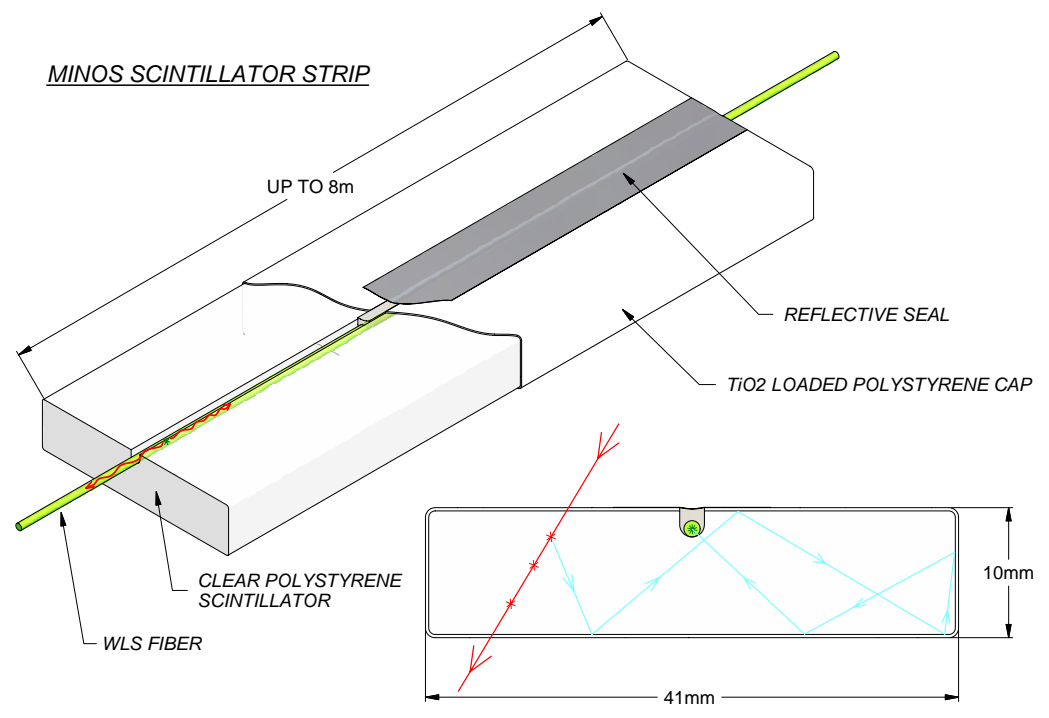

Figure 3.6: Drawing of a scintillator strip: the ionising particle produces light in the scintillator strip. The light is reflected by the reflective coating and is absorbed by the wavelength-shifting fibres, which brings the signal to the photomultipliers. The figure is taken from [81].

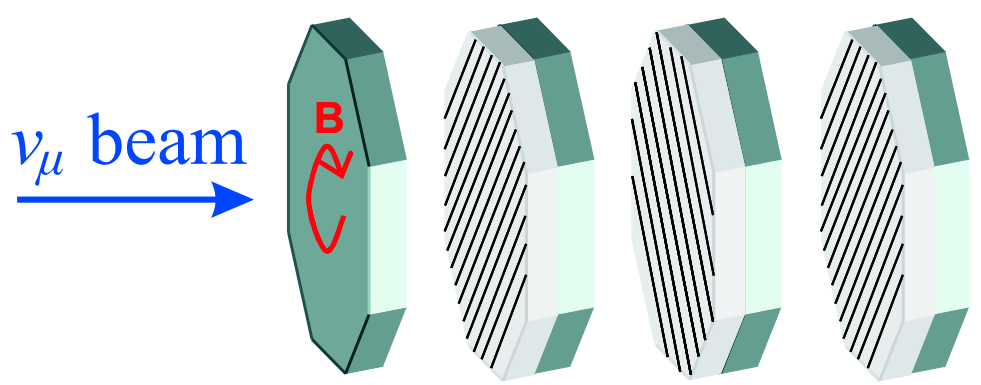

Figure 3.7: A cartoon of a MINOS detector sampling calorimeter showing the active scintillator strips alternated to steel planes. The scintillation strips on successive planes are oriented $90^{\circ}$ in respect to each other to allow 3D tracking. The figure is taken from [22]. 
tomultipliers and the electronics over time. UV Light Emitting Diodes (LEDs) are used to illuminate the wavelength-shifting fibres of the scintillating strips; the LEDs are mounted on racks that are called "pulser boxes". Optical fibres carry and inject the light from the LEDs to the scintillating modules manifold: in this way the light injection pulses fake the light from the scintillating strips and the signal can be read out by the photomultipliers and electronics chain. Information about the true light emitted by the LEDs is obtained by reading PIN photodiodes directly.

\subsubsection{The Near Detector}

The Near Detector is positioned $1 \mathrm{~km}$ away from the NuMI target and $110 \mathrm{~m}$ below ground. The overall mass of the detector is 980 metric tonnes and it consists of 282 steel+scintillator planes. Given the typical beam intensity at the Near Detector, an average of 16 events are collected at the Near Detector per spill trigger. The neutrino rate at the Near Detector justifies the smaller size of the Near Detector compared to the Far Detector. The Near Detector is a "squashed" octagon which is $6.2 \mathrm{~m}$ wide and $3.8 \mathrm{~m}$ high. The first 121 planes form the calorimeter part of the detector, which is used to detect the vertex of the interaction and measure the hadronic energy deposited. The steel planes are all identical, but the amount of scintillator varies from plane to plane. In the calorimetric part of the detector, steel planes are usually instrumented with 64 scintillator strips per plane. This guarantees coverage of the core region of the detector where the neutrino interactions are expected. Every five planes, the scintillator module extends further on the side (for a total of 96 strips per plane) to provide extra information about edge and veto activity: those planes are referred to as "fully instrumented" planes. Figure 3.8 shows the geometry for the Near Detector planes (on top for each of the two views) and for the fully instrumented planes (at the bottom for each of the two views). Each scintillating strip is read out individually in the calorimeter, one side only mirrored at the other end. The remaining planes at the back of the detector form the spectrometer and they are meant to track long muons: only 
one every five planes is instrumented with a scintillator plane. The wavelength-shifting fibres from four strips are read out in a single electronic channel. The ambiguity is resolved offline by the reconstruction algorithm.

The photomultipliers used at the Near Detector are 64-anode Hamamatsu multi-pixel photomultipliers. The read out electronics is designed not to have dead time. The digitisation is continuous at a rate of $53 \mathrm{MHz}$ (the frequency matches the beam radiofrequency and corresponds to digitising every $18.8 \mathrm{~ns}$ ). The digitisation is performed by a Charge Integration Encoder (QIE) Analog-To-Digital Converter (ADC) chip. The signal is digitised by comparing it to successive ranges. Each QIE chip resides on a MENU board. 16 MENUs are locate in a MINDER, with 4 MINDERs needed for each 64-anode photomultiplier. Up to 8 MINDERs are located in a MASTER. The linearisation of the signal and the pedestal subtraction are performed in a MASTER using lookup tables, which have been filled using the charge injection calibration at each MENU.

The acquisition is started by two different class of triggers: the spill trigger starts acquisition synchronous with the NuMI spill. Data are digitised continuously for $13 \mu \mathrm{s}$, acquiring $1.5 \mu \mathrm{s}$ before the spill trigger not to truncate events. The other trigger mode is the dynode trigger, which is used for acquisition of cosmic rays and starts digitising the signal for 150 ns when a photomultiplier signal is above a programmable threshold.

A coil passing through each plane of the detector produces a $1.3 \mathrm{~T}$ magnetic field. The magnetic field, in the normal polarisation, is used to bend $\mu^{-}$towards the centre of the detector to measure the momentum from the curvature. In some data taking periods, the magnetic field has been reversed in order to focus $\mu^{+}$and provide a better understanding of the detector and of the beam.

\subsubsection{The Far Detector}

The Far Detector is located $735 \mathrm{~km}$ far away from the NuMI target and $705 \mathrm{~m}$ below ground. The Far Detector has a mass of 5400 metric tonnes, it is an octagon, 8 m wide. 

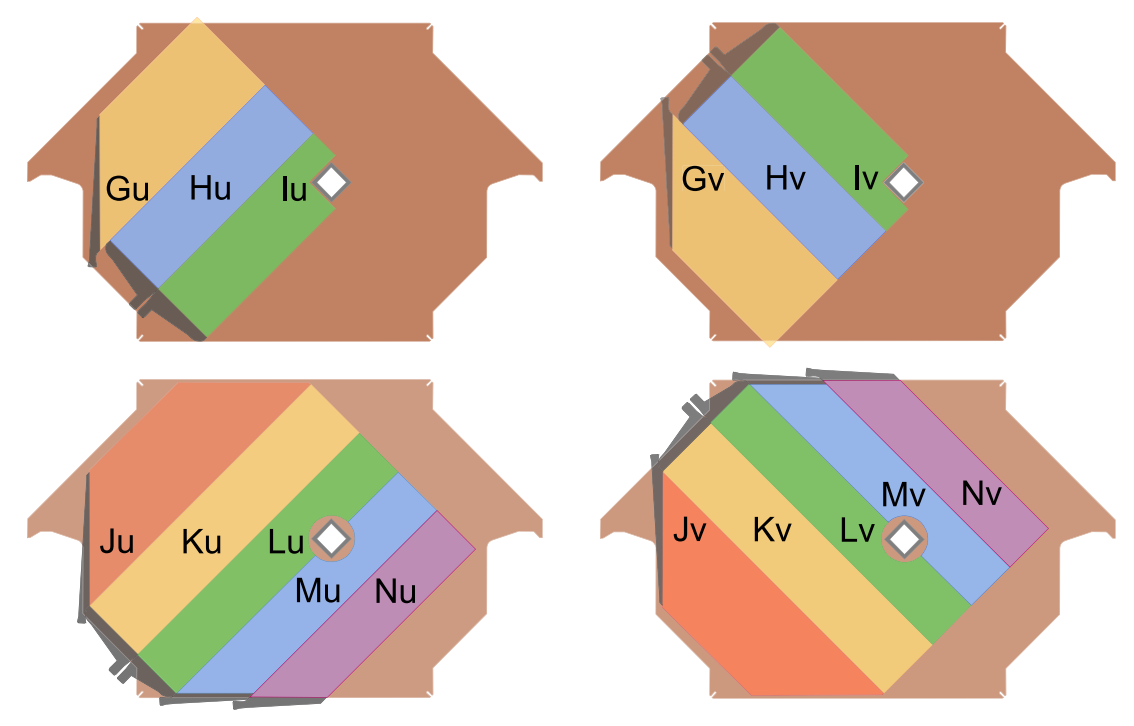

Figure 3.8: Scintillator arrangement for two Near Detector planes (top) for each of the two views, and for the fully instrumented planes (bottom) for each of the two views. Figure taken from [81].

It consists of 486 steel planes arranged into two supermodules (249 and 237 planes), which are separated by $1.1 \mathrm{~m}$. The coil is in the centre of the octagon and the magnetic field is $1.27 \mathrm{~T}$ induced in the steel planes. Figure 3.9 shows the scintillator module arrangement on a plane.

At the Far Detector, 16-anode photomultipliers are used. Eight scintillator strips are read out on the same anode. The ambiguity is resolved off line by the reconstruction algorithm. There is a separation of about a meter between fibres read out by the same anode and all strips are in the same plane. At the Far Detector, the electronic is optimised for a low-rate environment dominated by noise events. On the contrary, while at the Near Detector a timing resolution of $19 \mathrm{~ns}$ is good enough, a time resolution of $5 \mathrm{~ns}$ is required at the Far Detector to distinguish from up-going or down-going atmospheric neutrinos. The photomultiplier is read by a VA chip (VA stands for the initials of the ASIC chip reference number). Three VA chips are mounted on a VA Front-end Board (VFB). Each VFB houses a customised ASDLite chip which compares the signal from the dynode to a programmable threshold. Twelve VFBs are read out by a VA Readout Controller (VARC), which is in charge of the digitalisation of the signal, if the threshold set by the ASDLite is passed. To 


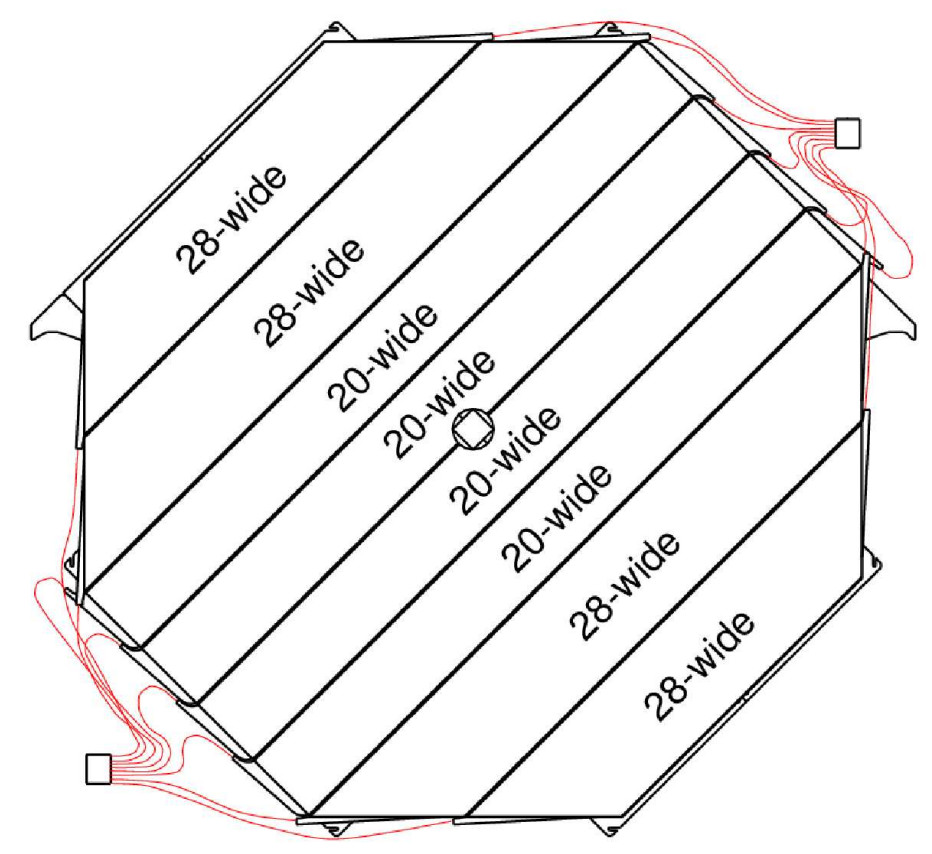

Figure 3.9: The arrangement of the scintillator modules on a Far Detector plane. The outer modules consists of 28 scintillating strips, while the internal modules consists of 20 scintillating strips each. Figure taken from [81].

reduce the rate of noise events, a " $2 / 36$ " trigger is used: events are digitised only if at least 2 dynodes in different photomultipliers in the same VARC receive a signal within 400 ns. A VARC also produces a timestamp for the recorded activity.

Every time there is a spill trigger, the signal is transmitted from the Near Detector over the Internet and the acquisition of events lasts for $100 \mu \mathrm{s}$, with $30 \mu \mathrm{s}$ before the trigger also acquired to avoid to truncate events. Out of spill, the Far Detector is read out using other trigger logic as the rate of noise is too high to digitise continuously. To trigger on cosmic rays and atmospheric neutrinos, the acquisition is started when 4 contiguous planes out of 5 record some signal or 4 planes record a signal exceeding the threshold of 1500 ADC counts or 20 planes record activity in the detector. Fake spill triggers are also used to record the noise which would be in real spill triggers on top of any neutrino signal. The trigger rate at the Far Detector is $30 \mathrm{~Hz}$. 


\subsubsection{The calibration detector}

The MINOS Calibration Detector was functionally identical to the Near Detector and Far Detector. The Calibration Detector has been useful to determine the calibration procedure for the MINOS main detectors. Between 2001-2003 it was exposed to a CERN beam of mixed composition (e, $\mu, \pi$ and $\mathrm{p}$ ) with momentum $0.2-10 \mathrm{GeV} / c$ : the results are used in MINOS to fix the absolute energy scale. Description of the detector is available in [88]. It consisted of 60 planes, with the same geometry as the main MINOS detectors. The Calibration Detector was not magnetised and the thickness of the steel was $2.50 \mathrm{~cm}$. To mimic the main detector readout conditions, one side was read out by the same readout chain as the Far Detector, while the other end was read out in the same way as the Near Detector. The description of the results of the comparison between the two read out systems can be found in [89]. As the difference between the two detector readouts are fully simulated in the MINOS MC, the impact of the differences is reduced.

The resolution of the hadronic shower has been determined to be $56 \% / \sqrt{E(\mathrm{GeV})} \oplus 2 \%$. Figure 3.10 shows the energy resolution for different particles as a function of the particle energy $E$. A part of the absolute shower energy systematic error in MINOS accounts for the residual differences from the Calibration Detector MC and the test beam data.

\subsection{The MINOS Monte Carlo}

The NuMI beamline is simulated using the FLUKA [91] simulation package to predict the hadron production from the target and in the decay pipe. The full beamline geometry is simulated using the Flugg package [92] which handles the GEANT4 geometry in FLUKA. Run I (May 2005 - February 2006), Run II (September 2006 - July 2007) and Run III (November 2007 - June 2009) are simulated each with the appropriate target position. Helium in the decay pipe is simulated for Run III as well. Documentation on the latest NuMI beam simulation is available in [93].

The neutrino fluxes are predicted by the beamline simulation and they are used as an 


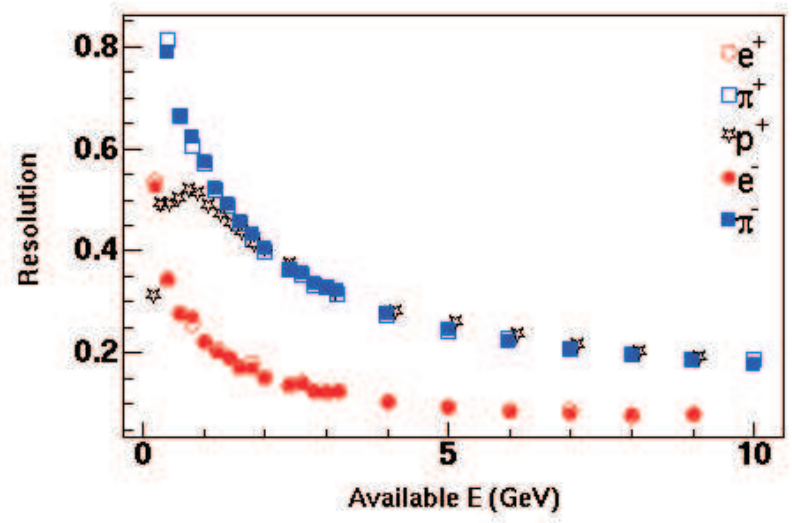

Figure 3.10: The figure shows the energy resolution as a function of the energy for different particle types from the Calibration Detector. The overall energy resolution assigned to the hadronic shower energy has been found to be $56 \% / \sqrt{E} \oplus 2 \%$ in agreement with the single pions and protons resolution. The plot is taken from [90].

input to the following step of the simulation which deals with the neutrino interactions, which are simulated using NEUGEN-v3 [94]. The neutrino cross section accounts for quasielastic, resonant, deep-inelastic neutrino interactions. The MINOS detector is simulated using the geometry offered by GEANT3 [95], which is responsible to propagate the neutrino interaction products in the detectors. The propagation of particles is obtained using the GCALOR package [96]. An overlay of the muons from neutrino interactions in the rock surrounding the detector with neutrino events in the detector is performed. Once the energy of the particle has been deposited in the detector, the scintillator, photomultiplier and electronic readout are simulated using two specific MINOS packages called PhotonTransport and DetSim. DetSim assigns a random date to the events among the data taking period: real calibration constants from that date are used in the MC to assure uniformity with the data. 


\subsection{Event Reconstruction}

The main aim of the MINOS reconstruction is to provide a good performance on the reconstruction of muons. As muons usually travel far in the detector, the reconstruction algorithm tries to identify the track that the muon has left. The remaining activity that is not classified as a track is combined into showers. Tracks and showers are the reconstruction objects that are then combined into neutrino events ${ }^{1}$.

As the rate at the Near Detector is high, the reconstruction algorithm applies some preliminary cuts to isolate hits that are close in space and/or time (this is done by looking for spatial or temporal gaps among hits). The clusters of hits in the same space/time are referred to as slices. The algorithm tries to form tracks and showers only from hits within the same slice.

Tracks are formed from clusters of hits which lie on successive planes. A Kalman Filter [97] estimates the actual path of the tracks which are bent by the magnetic field. The track fitter evaluates the particle momentum from the curvature and, if the track is fully contained in the detector, from the range as well.

The shower algorithm clusters hits that may have originated from the same neutrino interaction. Given the nature of the multianode photomultipliers, it is not unlikely that a photo-electron migrates from one anode path to another, causing a signal on multiple anodes (cross-talk). Despite improvements in the simulation and understanding of the data crosstalk $[98,99]$, the data/MC agreement for very low pulse height hits is not very good. Real minimum ionising particles produce a signal which is about 7 photo-electrons. To remove the low pulse height hit discrepancy in data and MC, all hits below 2 photo-electrons are removed from the shower formation in data and MC to improve the agreement. The shower energy is obtained by sum of all the pulse height energy.

The last stage of the reconstruction combines tracks and showers to form events, which may be composed of only tracks or only showers or both tracks and showers. The event

\footnotetext{
${ }^{1} \mathrm{~A}$ very good, not completely up-to-date documentation is available at the URL: http://wwwnumi.fnal.gov/offline_software/srt_public_context/WebDocs/reconstruction/RecoSRDoc.htm
} 


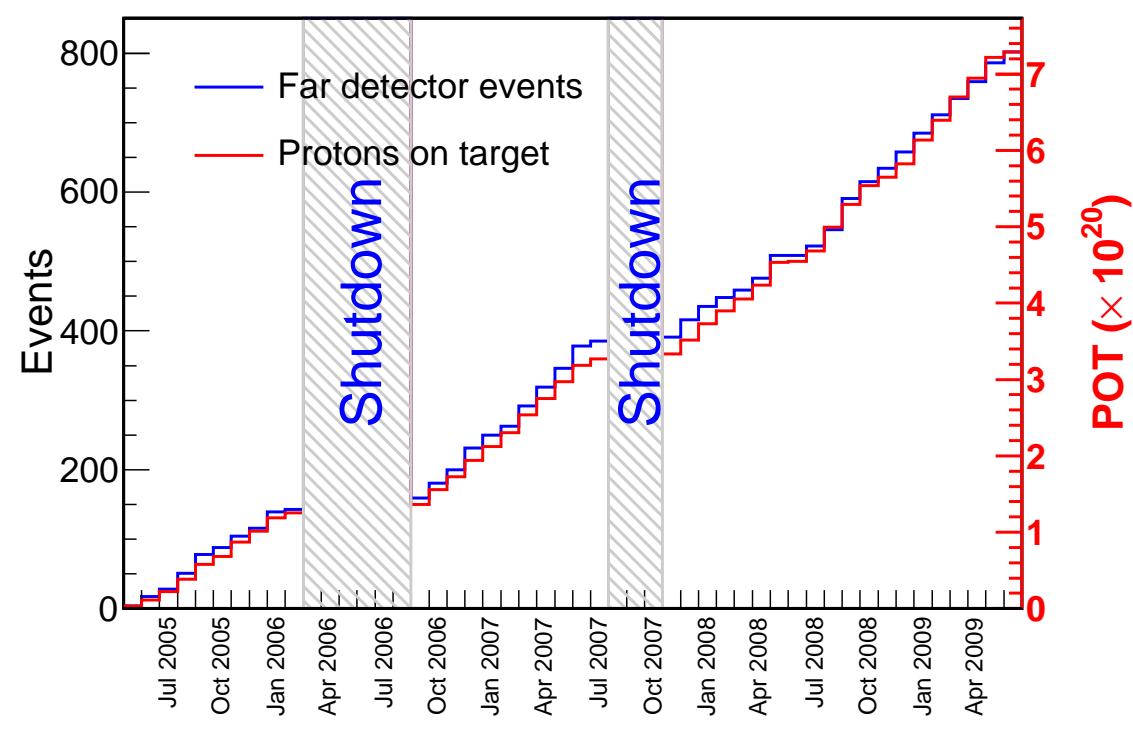

Figure 3.11: The integrated number of protons on target (red) as a function of time and the integrated number of neutral current events (blue) at the Far Detector. The three runs analysed in this thesis are visible as they correspond to a full data taking period between the accelerator shutdowns. The number of neutral current events selected at the Far Detector is an anticipation of the results obtained in chapter 8.

making algorithm tries to find the vertex from which the event originated. The overall event energy is the sum of single tracks and showers which compose the event.

\subsection{Dataset analysed}

This thesis analyses the data collected at the Near Detector and Far Detector in the three run periods between March 2005 and June 2009. Only the LE010z185i beam configuration is used and, at the Near Detector, only data collected with the magnetic field optimised to focus $\mu^{-}$are used. The overall protons on target collected with those configurations at the Far Detector are $7 \times 10^{20}$, slightly less $\left(\approx 5.5 \times 10^{20}\right.$ protons on target $)$ at the Near Detector once downtime and inverted field running are excluded. Figure 3.11 shows the integrated protons on target at the Far Detector in red, as well as the integrated number of selected neutral current events at the Far Detector in blue. The number of neutral current events selected at the Far Detector is the result of the analysis performed in chapter 8 . 


\section{Chapter 4}

\section{The detector calibration in MINOS}

This chapter presents the reasons why detector calibration in MINOS is necessary. The steps in the calibration chain used in MINOS are discussed. Section 4.3 is dedicated to the drift calibration technique, which has been developed in this thesis. Performances of the detector response versus time and temperature are discussed, as well as the systematic errors associated with the drift calibration.

\subsection{Detector calibration}

For charged current events, the energy of the neutrinos interacting in the detectors can be reconstructed from the sum of the energy of the muon track (from curvature or range) and of the energy of the hadronic shower (from calorimetry). For neutral current events, the energy of the outgoing neutrino cannot be measured and the only visible energy deposition corresponds to the shower energy of the hadronic recoil. While the track energy measurement is quite robust, the shower energy measurement is more likely subject to mismodelling or inaccuracies that need to be corrected. The measurement of the shower energy through calorimetry is obtained by summing all pulse heights of the hits in the shower and scaling the resulting pulse height to the true shower energy.

In a neutrino oscillation analysis, an uncertainty on the resolution of the reconstructed 
neutrino energy, mainly due to the shower energy uncertainty, has an impact on the determination of the oscillation parameter $\Delta m^{2}$, which is determined by the horizontal position of the minimum of the oscillation probability, as shown in figure 2.2. Two different aspects of uncertainty on the shower energy cause uncertainty on $\Delta m^{2}$ : the relative shower energy measurement between detectors and the absolute shower energy. An absolute energy scale calibration is necessary to correctly compute the total energy from the combination of the shower energy and muon energy and its uncertainty coming from calibration is 5.7\% [100]. The absolute shower energy uncertainty is large at each single detector but its effect can be reduced by using the Near Detector neutrino visible energy spectrum to constrain the prediction of the unoscillated Far Detector visible energy spectrum, as will be shown in section 8.7. On the contrary, the relative knowledge of the shower energy between the Near Detector and the Far Detector cannot be reduced at analysis level and the detector calibration guarantees that data and MC for both detectors are normalized to the same energy. In particular, Near Detector and Far Detector data are compared to the MC independently [100]: the systematic error on the shower energy scale when comparing the Far Detector data to the $\mathrm{MC}$ is $0.9 \%$, the systematic error on the Near Detector shower energy scale between data and $\mathrm{MC}$ is $1.9 \%$. This gives a total Near Detector to Far Detector relative energy scale uncertainty of $2.1 \%$ when summed in quadrature.

\subsection{The calibration chain}

\subsubsection{The data calibration chain}

The steps to convert the raw detector hit pulse heights into calibrated pulse heights are described in [81]. The uncalibrated pulse height $P_{\text {raw }}(s, x, t, d)$ which depends on the particular strip $s$, position along the strip $x$, time $t$ and detector $d$ is multiplied by a sequence

of calibration correction factors to obtain the fully corrected signal $P_{\text {corr }}$ according to the 
following equation:

$$
P_{\text {corr }}=P_{\text {raw }} \times D(d, t) \times L\left(d, s, P_{\text {raw }}\right) \times S(d, s, t) \times A(x, d, s, t) .
$$

In detail, the corrections are:

- The drift correction $D(d, t)$ takes into account the photomultiplier gains, the electronics and the scintillator ageing and dependence on the temperature. The correction is evaluated using through-going cosmic muons. A more detailed description of the drift correction is given in section 4.3 .

- The linearity correction $L\left(d, s, P_{\text {raw }}\right)$ is necessary to account for the photomultiplier non linearity. This non linearity is $\approx 5-10 \%$ for light level above 100 photo-electrons [101, 102]. In addition, the Far Detector electronics has a non linear response at a similar signal size. The Near Detector photomultipliers also show non linearity at small pulse heights. The light injection system is used to correct all of these effects by injecting known pulses in a range from a few to hundreds of photo-electrons into the read out system. By comparing the measured pulse height from the detector readout to the known pulse height generated by the light injection system, a pulse height dependent correction factor is evaluated on a strip-by-strip basis. The light emitted by the LED system and monitored by a PIN diode system is known to be linear within 1-2\% itself [81]. At the Near Detector the non linearity is measured as a function of the true illumination measurement provided by the PIN diodes. At the Far Detector, the double-ended readout is used and exploits the attenuation of the signal along the strip. Both ends of the strip are read out, but the light is injected at one end at a time. At the far end of the strip, the signal is attenuated enough to be within the linear readout range. This allows an independent measurement of the true illumination.

- The strip-to-strip non-uniformity calibration $S(s, d, t)$ is obtained using through-going 
cosmic rays. The mean response at the centre of each strip to a muon of normal incidence is evaluated for each strip. Then, the mean response of each strip is normalised to the mean detector response for the same time period according to:

$$
S(s, d, t)=\frac{\text { Mean response of detector }(d, t)}{\text { Mean response of the strip end }(s, d, t)}
$$

This correction factor allows removal of channel-by-channel differences such as scintillator light yield, wavelength-shifting fibres collection efficiency, readout fibre attenuation, photomultiplier quantum efficiency and photomultiplier gain.

- The attenuation correction $A(x, d, s, t)$ is necessary as light attenuates as it travels along the wavelength-shifting fibre. A module mapper was used during detector construction for both detectors to illuminate the strips with $\gamma$ rays of well defined energy (662 keV from ${ }^{137} \mathrm{Cs}$ ). Measurements of the recorded read-out from the strip ends were made as a function of the position along the strip length where the strip was irradiated. The light output $R(x, d, s, t)$ can be parameterised as the sum of the readout from each single strip end $(1,2)$ attenuated by an exponential factor depending on the distance $x$ from each readout end:

$$
R(x, d, s, t)=R_{1}(d, s, t) e^{-x / L_{1}(d, s, t)}+R_{2}(d, s, t) e^{-x / L_{2}(d, s, t)}
$$

where $L_{1}, L_{2}$ are attenuation lengths. From the parameterisation above, it is possible to evaluate the correction factor $A(x, d, t, s)$ that needs to be applied.

\section{Inter-detector calibration and the absolute energy calibration}

While the previous corrections are meant to make the detector response flat over space and time, an extra multiplicative correction factor $M(d)$ on the result of equation (4.1) is 


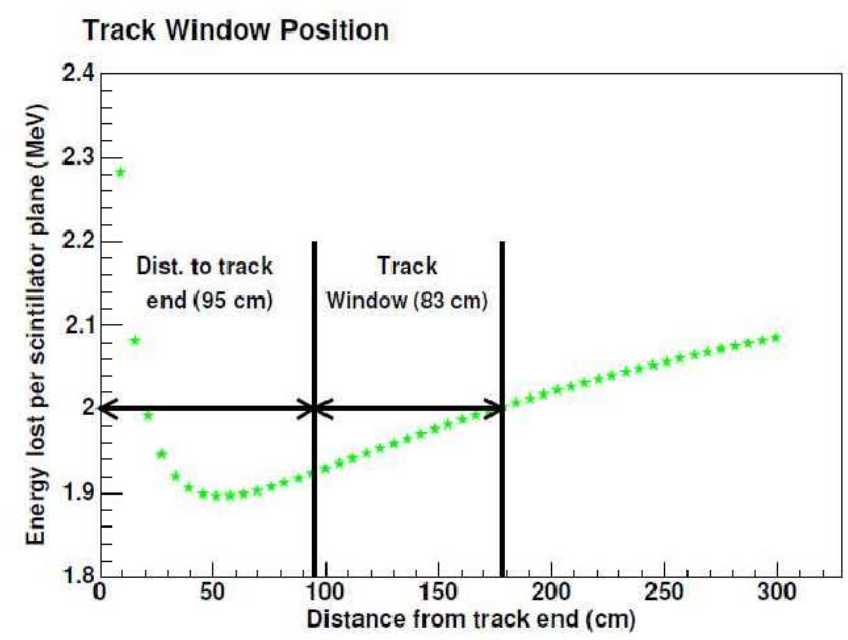

Figure 4.1: Illustration of the "track window" technique: cosmic muons are used and the energy deposited at a fix distance from the track end is used to calibrate data and MC to the same energy. The plot is taken from [103].

needed to calibrate the detectors to the same deposited energy:

$$
P_{\text {normalised }}=P_{\text {corr }} \times M(d)
$$

The energy deposited by muons in the detectors follows the Bethe-Bloch equation [12] and muons that stop in the detectors are abundant enough to be used to measure the energy deposition from range. A description of the Bethe-Bloch equation is given in section 6.1. The mean energy deposited by cosmic muons at a known distance from the track end is used to correct the pulse height into muon equivalent energy unit (MEU). This technique is referred to as the "track window" technique, as the window to calculate the energy deposited is meant to select the hits which are close to the minimum ionising stage [103]. Figure 4.1 shows the application of this technique. An extensive dataset is needed for this calibration stage, so all selected cosmic muons are used to give one MEU constant per detector, both for data and MC. With this technique, the relative calibration of the detectors is achieved at the $2.1 \%$ level. The same inter-detector calibration has been applied also at the MINOS calibration detector.

The final conversion of the detector pulse height into particle energy is achieved through 
the MINOS calibration detector [88] which was exposed to test beams of different particles at CERN. By knowing the beam momentum of the different particles, it has been possible to study the response of the detector to the absolute particle energy. The calibration to the absolute energy has been performed at the $5.7 \%$ level.

\subsubsection{The Monte Carlo calibration chain}

In principle the Monte Carlo follows the exact same calibration as the data, covering exactly the same time period as the data taking. The number of photo-electrons in the first place depends on the photomultiplier gains which follows Poisson statistics. While for the data, the drift correction takes into account a possible variation of the photomultiplier gains and electronics, in the MC, information about the actual photomultiplier gains has to be made available. During the data taking, the light injection system pulses the fibre from each strip end to monitor the stability and gain of each channel. At the Far Detector, the strip ends are each pulsed about 300 times an hour, while in the Near Detector each strip end is pulsed 1000 times per hour. By comparing the readout signal to the input light level (tuned to be 50 photo-electrons per pulse after the linearity correction), it is possible to evaluate the number of ADC counts per photo-electron and the relative Root Mean Square (RMS) of the distribution. The uncertainty on the gains is conservatively estimated to be $10 \%$ [104] from measurements from day-by-day variations and from the fit to the 1 photo-electron peak. It is expected that gain mismodelling affects low energy showers particularly. Low energy showers are the most important events in the neutral current analysis as a potential sterile neutrino signal would manifest itself as a lack of low energy neutral current events at the Far Detector; however, low energy showers are also the events that are more likely to be subject to data and MC discrepancies. Special MC studies varying the gains value by $\pm 1 \sigma$ have been performed to check the impact on the showers. The effect is below the systematic error quoted for the absolute calibration systematic error. 


\subsection{The drift calibration}

A good part of the work in this thesis has been devoted to improving the drift correction $D(d, t)$ in the calibration chain presented above. The original technique has been developed in [22] but it has been further studied here to make it more robust to bad detector conditions, change in trigger and differences in tracking efficiencies in more recent reconstruction versions. A complete consistent dataset of drift constants has been provided from Run I to Run III for the present reconstruction version. A fully automated production of daily drift constants has been developed and has been producing constants starting from Run IV on. Furthermore, the detector ageing and dependence on the temperature have been studied.

The detector response studied in the drift correction is a combination of the scintillator light output, the photomultiplier gains and the electronic readout. Any change in any of these three components correspond to a change in the detector response. The electronics is known to be fairly stable (studied in [105] at the Far Detector) but it is affected by hardware swaps. When a hardware swap is made, the electronics is recalibrated to minimally change the overall detector response. The change is also only local and does not affect the overall detector. The scintillator light output was studied by dedicated ageing tests [81]. Based on these tests, the non-reversible ageing due to yellowing of the scintillator and the attenuation of the wavelength-shifting fibres is expected to be $1.2 \%$ per year at $20^{\circ} \mathrm{C}$. A reversible loss in light output due to temperature effects has been quantified as $0.3 \%$ per ${ }^{\circ} \mathrm{C}$. The degradation of the wavelength-shifting fibre output is accelerated by the increase in temperature as well. From the photomultiplier gain measurement provided by the LI system, the long term variation of the gains due to ageing seem to be around $4 \%$ per year. From temperature effect studies, a gain decrease of $0.2 \%$ per $1^{\circ} \mathrm{C}[106]$ for the Far Detector 16 -anode photomultipliers and of $0.55 \%$ per $1^{\circ} \mathrm{C}$ (from figure 9 in [107]) for the Near Detector 64-anode photomultipliers are expected from dedicated tests.

The overall detector response is affected by the long term trend of scintillator light output, wavelength-shifting fibres, electronics and photomultiplier gains, as well as by short 
term effects like temperature variation or high voltage changes. Applying the drift correction $D(d, t)$ makes the detector response not dependent on ageing, temperature and voltage effects. The test-stand measurements for ageing and temperature effects are superseded by the "in situ" measurements presented in this chapter.

\subsubsection{Cosmic muons}

Cosmic muons are produced by primary cosmic particles (mainly single protons) interacting in the atmosphere to produce hadronic showers. The mesons (pions and kaons) in those showers decay to muons. From charge conservation arguments, the charge ratio is expected to be $\frac{N_{\mu^{+}}}{N_{\mu^{-}}} \approx 1.3$, but it is expected to be a function of the primary particle energy. There can be several competing processes that change the ratio: the pion to kaon ratio is not constant over the centre of mass energy, and the relative contribution of pions and kaons to the decay into muons is a function of energy. More energetic pions are expected to interact before decaying into muons, leaving the muon component coming from kaons to contribute more at higher energies. The muon differential production in the atmosphere has been parameterised [108] as:

$$
\frac{d N_{\mu}}{d E_{\mu, 0}} \approx \frac{0.14 \cdot E_{\mu, 0}^{-2.7}}{c m^{2} s \operatorname{sr} G e V} \cdot\left(\frac{1.0}{1+\frac{1.1 \cdot E_{\mu, 0} \cos \theta}{\epsilon_{\pi}}}+\frac{0.054}{1+\frac{1.1 \cdot E_{\mu, 0} \cos \theta}{\epsilon_{K}}}\right)
$$

where $E_{\mu, 0}$ is the surface energy of the muon and $\theta$ the zenith angle. The two terms in brackets represent the contribution to muon production coming from the pions (left) and the kaons (right). $\epsilon_{\pi}$ (equal to $115 \mathrm{GeV}$ ) and $\epsilon_{K}$ (equal to $850 \mathrm{GeV}$ ) represent the energies above which pions and kaons interact more often than decay. The $E_{\mu, 0}^{-2.7}$ dependency reflects the steeply falling cosmic ray flux.

Cosmic muons have been shown to provide an excellent source for monitoring the detector response over time. As explained in section 4.2.1, no absolute energy measurement is needed in the stage of correcting the detector response over time. This allows us to use cosmic muons, despite the difference in energy spectra and angular distribution at the 
two detectors. Muons travel through the rock overburden at the two detector sites and the amount of material is expected to be constant over time. The energy deposited by the cosmic muons is well modelled by the Bethe-Bloch equation. Simple cuts allow the selection of cosmic muons and exclude anomalous energy loss that can bias the deposited energy distribution (for example the increase of ionisation at the very end of the track). More details on the cosmic muon selection is given in section 4.3.2. The muon rate at the Near Detector is $\approx 12 \mathrm{~Hz}$ and at the Far Detector is $\approx 0.5 \mathrm{~Hz}$. The mean energies at the detectors (considering the angular distribution factor $\cos \theta$ ) are approximatively $64 \mathrm{GeV}$ at the Near Detector and $73 \mathrm{GeV}$ at the Far Detector.

A seasonal dependence in the number of muons at both detectors has actually been observed $[109,110]$. The change in the flux of muons is due to the seasonal change in the stratospheric temperature: a temperature increase causes a decrease in the density of the atmosphere, reducing the possibility for the secondary pions to interact before decaying. As a consequence, an increase in temperature manifests itself as an increase in the flux of muons. Nevertheless, despite this variation in the rate of muons over the time, no observable seasonal variation has been noticed in the median estimator. The energy loss by ionisation is quite flat in the muon momentum ranges of interest to the MINOS calibration, so small changes in the flux and energy of muons are expected to have a small impact on the average energy loss. Moreover, the median technique presented below is quite robust to changes in the detector response. The statistical precision of the drift calibration is approximately 0.5\%. Any change in the daily average detector response smaller that the statistical error cannot be resolved. For those reasons, the seasonal variation of the energy spectrum of the cosmic muons is believed not to affect the drift calibration, but dedicated MC studies will be performed in the near future by the MINOS Working Group which studies cosmic ray physics. 


\subsubsection{The drift calibration technique}

The drift calibration uses all available cosmic muons (i.e. out of spill) that pass some criteria to monitor the detector responses over time. Cosmic muons provide a constant source of ionising particles in both detector locations. The median of all plane pulse heights on all planes from all muons over a period of 24 hours provides a good observable for monitoring the detector response over a day. In the MINOS Calibration Detector, the drift correction was evaluated using the light injection system. The same technique was initially adopted for the main detectors: correction for gain, electronics and temperature was performed every 3 hours at the Far Detector and hourly at the Near Detector. Nevertheless, after considerations in [111], the study of the detector response with cosmic muons was chosen as it monitors photomultiplier gains and electronics (like in the light injection technique) and it is also sensitive to variation in the scintillator light emission. While the light injection correction method provides some spatial dependent correction, the cosmic muon statistics requires a sampling over the whole detector, providing a single correction for the entire detector. This point will be discussed further in the course of this chapter. Studies performed in [22] have shown that a daily correction through cosmic muons is enough to remove drift and temperature effects.

\section{The event selection}

The selection of cosmic muons requires a single reconstructed track (no requirement is made on the presence of a shower). Activity recording is triggered by a hit in 4 out of 5 consecutive planes but not by a spill trigger. Figure 4.2 shows a typical cosmic muon event display at the Far Detector.

Even in the case of background passing the selection cuts, consistency of the background over time means it is not critical for the drift calibration purposes. Background that could be misidentified as cosmic muons is given by detector/instrumentation generated events. Those background events need to be removed as they are most likely not a constant effect 


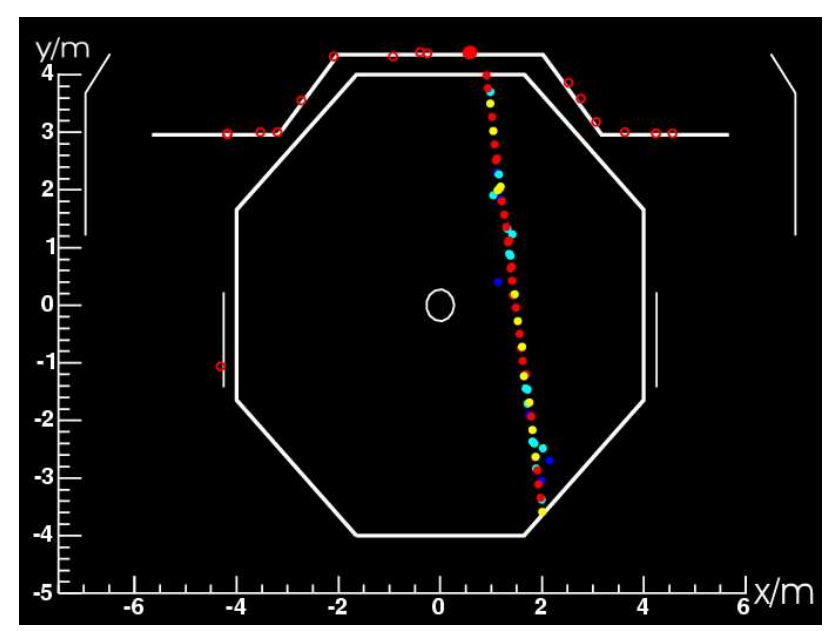

Figure 4.2: A typical cosmic muon as reconstructed by the on-line software at the Far Detector, in the $x y$ coordinate view. The detector shape is superimposed. Hits on the veto shield system above the detector are also visible. The figure is taken from [22].

over time. Particularly, light injection events need to be removed as they heavily bias the energy deposition. Light injection events are removed using a flag which is set when the light injection is flashing. At the Far Detector some specific standard cuts, presented in [21] and described in section 8.4.2, are applied. Some extra requirements on the high voltage status, coil current status and criteria to guarantee the stable detector conditions are required according to $[112,113]$.

\section{Track selections}

Tracks need to be at least 9 planes long at the Far Detector and 15 planes at the Near Detector. The number of planes required at the Near Detector has been changed from its original value in [22] to be more stringent as a hardware trigger change happened on the number of planes required to trigger activity acquisition at the end of 2005. The trigger change caused a difference in the acceptance of cosmic muons between the old and new trigger.

As shown in figure 4.3 the number of muons passing the selection is on average constant at the Near Detector and at the Far Detector. The number of muons selected is a quantity quite sensitive to detector generated events: noisy channels and light injection events that 

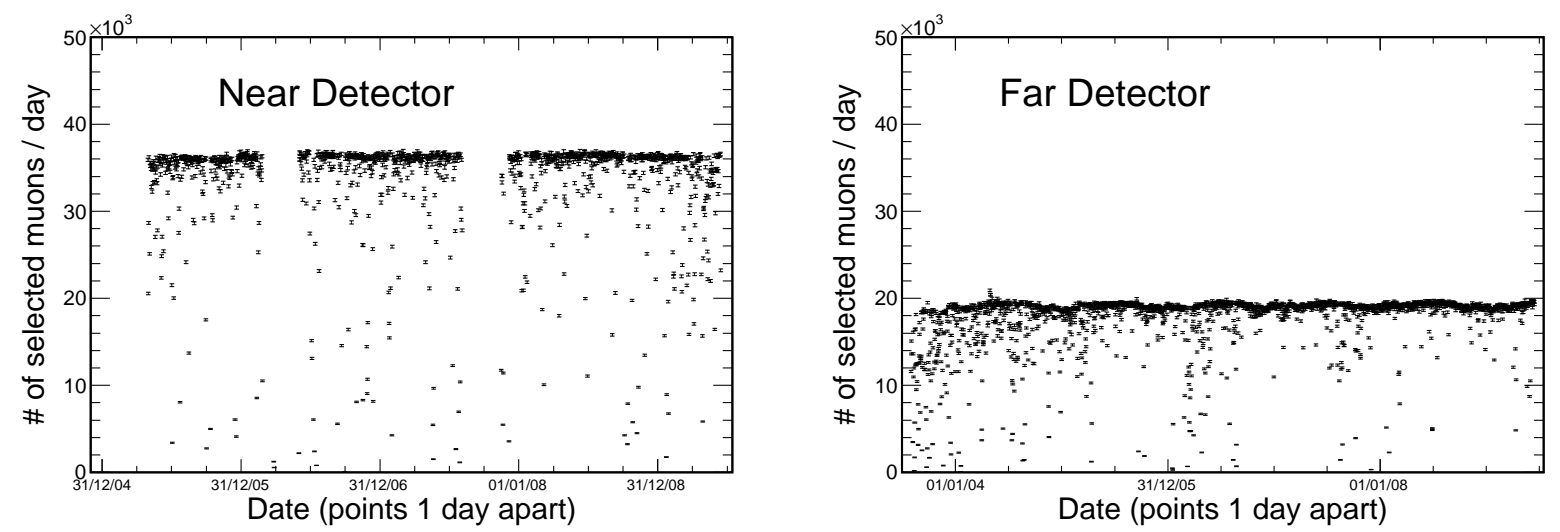

Figure 4.3: The number of muons per day passing the selection cuts described in the text. On the left is for the Near Detector, while on the right is for the Far Detector. At the Near Detector only $1 / 16$ of the events triggered by the consecutive plane activity are processed by the reconstruction. To maintain the number of muons at the Near Detector constant over the data taking time period a more stringent requirement on the number of planes crossed by the muon track has been necessary to guarantee the same acceptance after a hardware trigger change in December 2005. At the Far Detector, during the cosmic muon data acquisition but before the beam data taking started in March 2005, light injection events could be reconstructed as data events, making the requirements for light injection rejection cuts necessary.

are mistaken for physical events manifest themselves by increasing the rate of selected events. After applying the data quality cuts described, no anomalies are visible any more ${ }^{1}$.

The cosmic muons selected are energetic enough to be considered not deflected by the magnetic field to first order. The direction of the track in respect to the horizontal $(\cos \theta)$ can be evaluated as the ratio between the length along the horizontal direction and the track fitted path length. A track path-length correction factor (equal to $\frac{1}{\cos \theta}$ ) is required to be greater than 1.0 and less than 3.0. This is to cut out tracks steeper than $\approx 70^{\circ}$ (closer to the vertical). The reasons to exclude very steep cosmic muons is that the calibration needs to be optimised for events that are collinear to beam events that arise from neutrinos with a momentum along the $z$ direction. For the same reason, both the magnetic field and the track reconstruction are optimised to reconstruct mainly horizontal tracks. Each hit

\footnotetext{
${ }^{1}$ Some days in figure 4.3 show a lower number of selected cosmic muons: this is due either to detector downtime, or bad data quality or processing failure of the data files.
} 
along the track has its pulse height corrected by the path-length correction factor. This is to account for the difference in deposited pulse height whether the muon was crossing the plane orthogonally or with an inclination.

\section{Energy deposited per plane}

The first and last planes hit in each track are removed from the considered hits. This accounts for anomalous energy loss at the end of the track. The pulse height weighted mean position $\langle x\rangle_{p h}$ and $\left\langle y>_{p h}\right.$ of all the hits $i\left(x_{i}, y_{i}\right)$ on the same plane with pulse height $P_{i}$ are evaluated as:

$$
<x>_{p h}=\frac{\sum_{i} x_{i} P_{i}}{\sum_{i} P_{i}} \quad\left\langle y>_{p h}=\frac{\sum_{i} y_{i} P_{i}}{\sum_{i} P_{i}}\right.
$$

A coarse fiducial volume cut is applied on the pulse height weighted mean position $\langle x\rangle_{p h}$ and $\langle y\rangle_{p h}$ in each plane. In particular a radial distance from the centre of the coil is defined as:

$$
<r>_{p h}=\sqrt{<x>_{p h}^{2}+<y>_{p h}^{2}}
$$

In both detectors, the requirement on the pulse height weighted mean radial distance has to be more than $30 \mathrm{~cm}$ away from the centre of the coil hole. At the Far Detector the cluster has also to be within a $3.5 \mathrm{~m}$ radius circle centred in the coil. At the Near Detector the cluster mean position has to be within a $1 \mathrm{~m}$ radius from the beam spot and in the first 120 planes of the detector (calorimeter). On each day, the information of the deposited pulse height in each plane is recorded into a histogram. The median of the pulse height per plane deposited by orthogonal crossing cosmic muons has been shown [22] to provide a good indication of the detector response on a particular day. The error associated to this quantity is evaluated as the error on the mean, i.e. the RMS of the distribution divided by the square root of the number of plane entries. Technically this is not perfectly correct, as the error on the median should differ from the error on the mean. As an analytical 

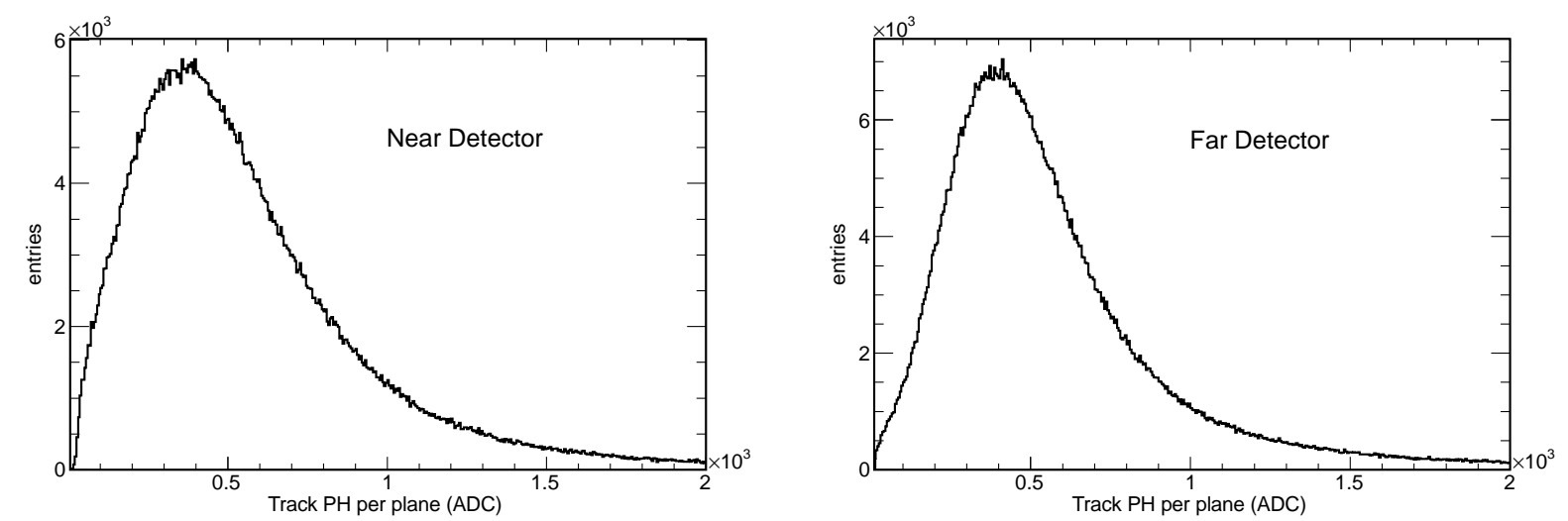

Figure 4.4: Distributions of the pulse height per plane deposited by selected cosmic muon tracks in the Near Detector (left) and in the Far Detector (right).

expression on the error on the median is not available, the error on the mean has been chosen as the quoted error for the drift constants. Figure 4.4 shows the distributions of the pulse height per plane (measured in ADC counts) deposited by selected cosmic muon tracks in the detectors. The ionisation per plane is expected to follow a Landau distribution [114], characterised by a long tail at high ionisation. The median provides a good estimator of the daily detector response as it is only moderately affected by the Landau tail in comparison to the mean estimator.

\section{The detector drift and the correction}

Every day, when data is available, the median pulse height per plane deposited by cosmic muons is recorded. This allows us to track the detector drifts over the data taking period. A particular detector response has been chosen as the reference response $m_{t_{0}}$ to which all the median detector response per plane will need to be normalised to. By convention the chosen reference constant is the median detector response per plane at 00:00:00 on $1^{\text {st }}$ December 2005. Once the median response per day $m_{t}$ at each day $t$ has been evaluated, the calibrated pulse height $\left(p h_{\mathrm{c}}\right)$ for the particular day $t$ is obtained by applying a correction factor $\frac{m_{t_{0}}}{m_{t}}$ to the uncalibrated pulse height $\left(p h_{\mathrm{unc}}\right)$ :

$$
p h_{\mathrm{c}}=p h_{\mathrm{unc}} \frac{m_{t_{0}}}{m_{t}}
$$


The drift correction factor, as well as the other calibration factors, are applied to each single hit in the detectors. Then a full reprocessing is needed in order to have completely calibrated data and Monte Carlo samples.

Figure 4.5 shows the detectors response drift over time. The Far Detector shows a consistent drop in response of approximately $2 \%$ a year. The discontinuity in detector response in March 2006 is due to a retuning of the voltage across the photomultipliers. At the Near Detector the detector response was stable for nearly one year before starting decreasing with time. The period of constant response is consistent with the photomultiplier gain variation over time and the scintillation light drift compensating, as will be shown in section 4.4 .

\section{Spatial dependence of the drift constants}

As already explained, the cosmic muon drift calibration technique provides a single constant for the whole detector. As the detector average drift is used to correct each single part of the detector, some parts will be under corrected and some other parts will be over corrected. The strip-to-strip correction $S(d, s, t)$ described in section 4.2.1 is responsible for normalising the response in different detector parts to the mean detector response at the same time.

Nevertheless, the median pulse height per plane deposited in different parts of the detectors is evaluated as a cross-check. As both the detectors are divided in two parts, this naturally offers two nearly independent detector areas on which to cross check the drift constants. At the Near Detector the detector is divided in the calorimeter and the spectrometer, while the Far Detector is divided into the two supermodules. The same technique to evaluate the median pulse height per plane is adopted, but only the planes in each of the single detector parts are considered each time. Figure 4.6 shows the median pulse height per plane deposited by muons in the whole Far Detector, compared to the one deposited in the two independent parts. At the Near Detector the calorimeter is the part where is important to have good calibration. For this reason it has been chosen to 

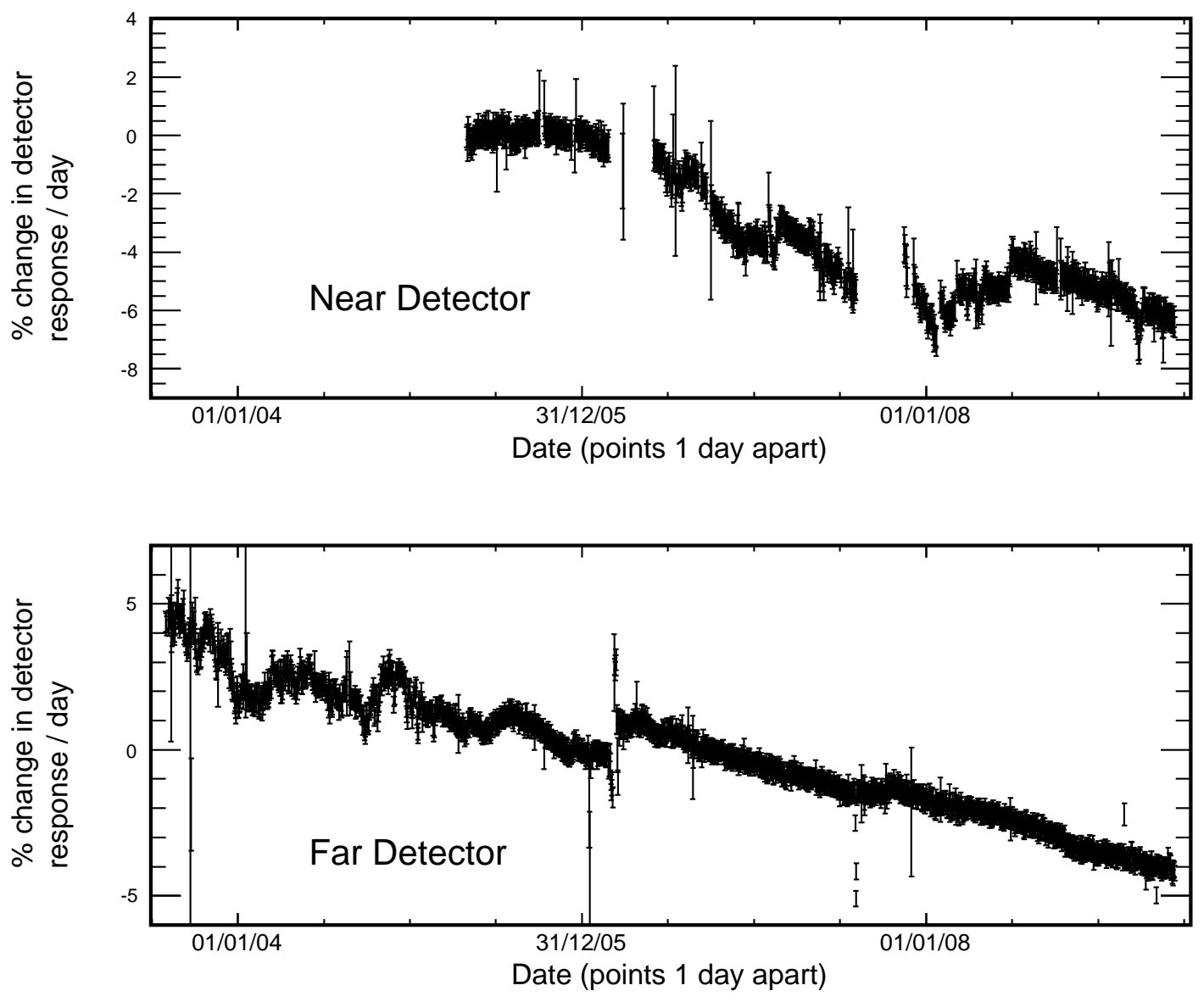

Figure 4.5: The percentage variation in detector response over the cosmic muons data taking time period. The top plot is for the Near Detector, while the bottom is for the Far Detector. The Near Detector became operational nearly two years after the Far Detector. The two periods without data taking at the Near Detector correspond to the beam and detector shutdown. The Far Detector remains operational during shut down periods mainly to collect atmospheric neutrino data. The change in detector response at the Far Detector in March 2006 corresponds to the photomultiplier tuning. 

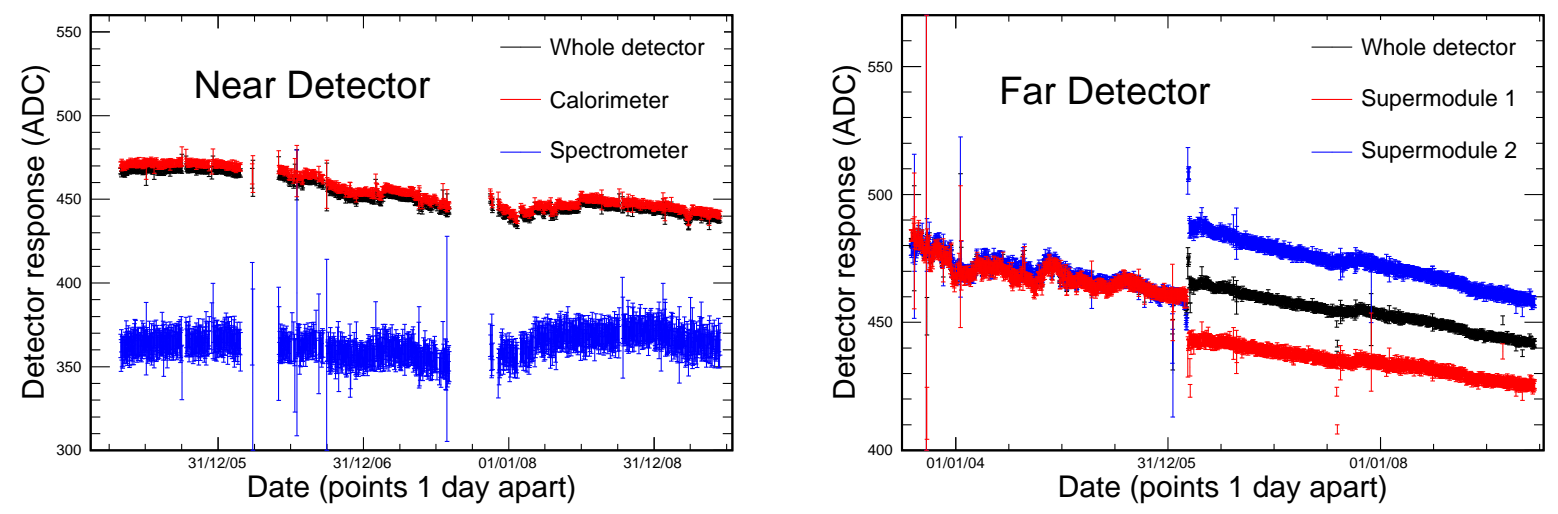

Figure 4.6: The drift constants for the whole detector and for two different parts of the detectors. The plot on the left is for the Near Detector and on the right for the Far Detector.

normalise the detector response to the calorimeter response. At the Far Detector, instead, the two supermodules have the same relative weight. So it is important to cross-check that the response in the detector does not show spatial dependence.

Figure 4.7 shows the median pulse height per plane deposited in the whole Far Detector and in the two supermodules after applying the linearity correction $L\left(d, s, P_{\text {raw }}\right)$, the drift correction $D(d, t)$ (on the left) and after applying the linearity correction $L\left(d, s, P_{\text {raw }}\right)$, the drift correction $D(d, t)$ and the strip-to-strip correction $S(d, s, t)$ (on the right). After applying the linearity and the drift correction the median detector response is flat but the two supermodules still show spatial variations. The strip-to-strip calibration almost completely removes the local differences, but some small differences are still present due to the different photomultiplier voltage condition before and after March 2006. The median detector response shows a discrepancy of the order of $0.5 \%$ between the data before and after the photomultiplier tuning for nearly $1 / 4$ of the data-taking time period. This discrepancy is taken into account in the evaluation of the systematic errors for the MEU number and in any case is well below other important systematics. Nevertheless, in the next full reprocessing it will be investigated and corrected. Given the symmetry between the two supermodules, as they are evenly sampled, the residual spatial discrepancy does not affect the MEU evaluation. Indeed, the individual neutrino events could be assigned the wrong energies, but on average these errors will cancel if the detector is evenly sampled. Using a single 

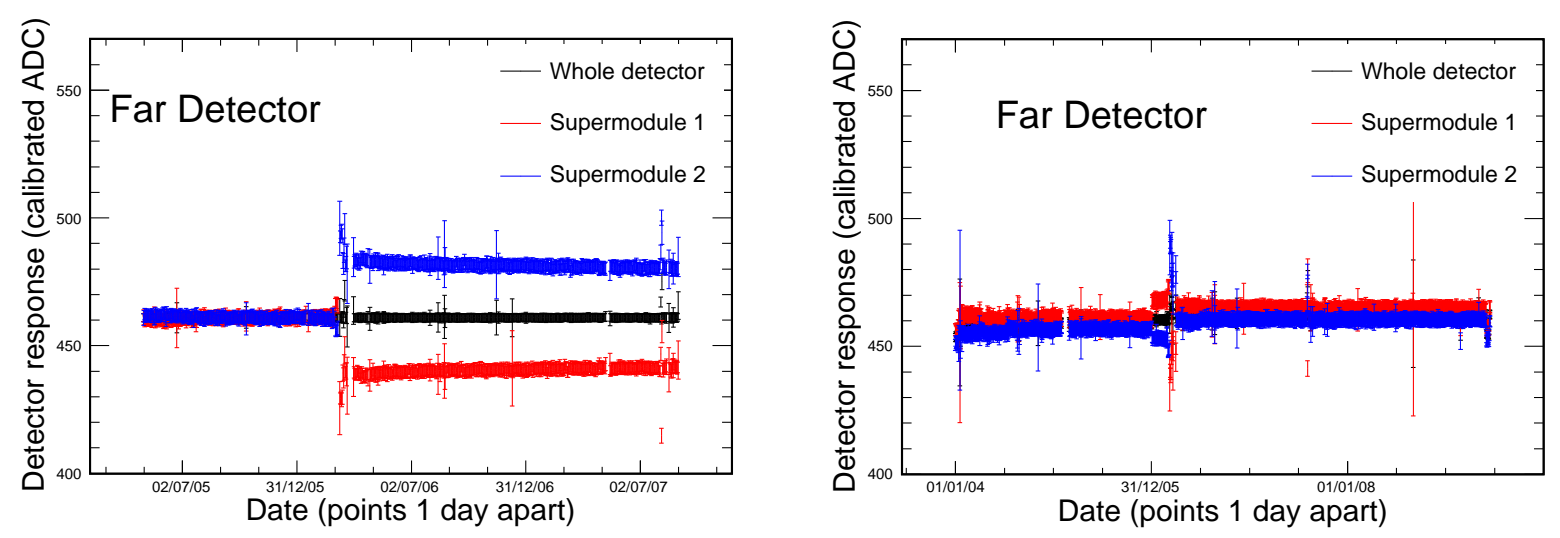

Figure 4.7: The median detector response for the whole detector and for the two supermodules after applying the linearity and the drift corrections (left) and after applying the linearity, the drift and the strip-to-strip corrections (right). Linearity and drift corrections remove ageing and temperature effects. The strip-to-strip correction removes spatial differences.

average constant for the detector might affect the energy resolution, but does not create an energy scale systematic error.

\subsubsection{The drift calibration systematic errors}

The full drift calibration constants are used to calibrated the detectors to the same MEU scale as explained in section 4.2.1. By construction, randomly wrong drift calibration constants would not affect the MEU calculation, but would have an impact only on the energy resolution. The only way the drift constants can have an effect on the MEU number evaluation is if the drift constants show a time dependent systematic error. Indeed, if this is the case, the errors would not cancel out.

Successive steps in the calibration chain are calibrated using the mean pulse height per plane instead of the median pulse height per plane. Thus, it is important to cross-check the consistency between the two variables. The systematic errors on the drift calibration are evaluated by comparing the correction factor $\frac{m_{t_{0}}}{m_{t}}$ in equation (4.8) as obtained by the median to the correction factor obtained by choosing the mean as the drift constant $\frac{m_{t_{0}}^{\prime}}{m_{t}^{\prime}}$. To evaluate the ratio between the two correction factors, we need to normalise the reference constants $m_{t_{0}}$ and $m_{t_{0}}^{\prime}$ to the same physics. While a complete freedom in the choice of the 

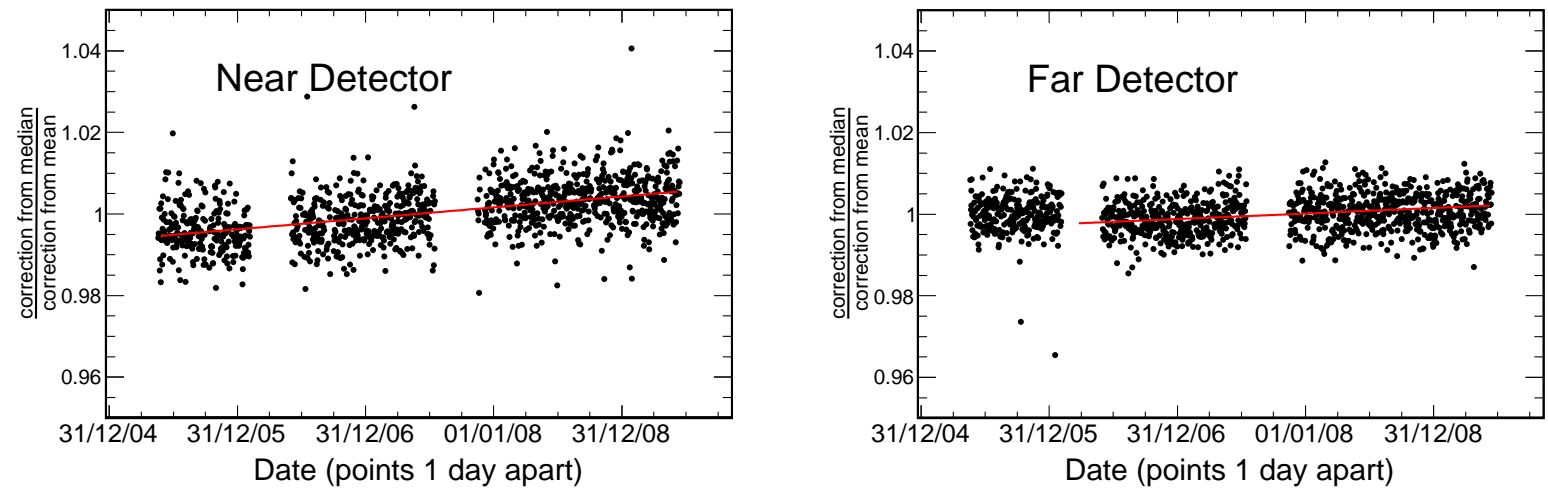

Figure 4.8: The ratio of the drift calibration correction factors from the median divided by the ones from the mean as a function of time for the Near Detector (left) and the Far Detector (right). The trend of this ratio has been used to find the systematic errors.

normalisation factor $m_{t_{0}}$ is given, the choice of an alternative reference constant $m_{t_{0}}^{\prime}$ is not arbitrary any more. The expected mean pulse height per plane $m_{t_{0}}^{\prime}$ corresponding to the choice of $m_{t_{0}}$, can be more correctly evaluated as:

$$
m_{t_{0}}^{\prime}=m_{t_{0}}\left\langle\frac{m_{t}^{\prime}}{m_{t}}\right\rangle
$$

where $\left\langle\frac{m_{t}^{\prime}}{m_{t}}\right\rangle$ represents the average value of the ratio of the daily mean pulse height per plane and the median pulse height per plane.

Figure 4.8 shows the ratio between the correction from the median and the correction from the mean as a function of time for the Near Detector and the Far Detector. In the Near Detector a clear linear trend over time is visible. A linear two-parameter fit is made to the data in figure 4.8. Comparing the values of the fit at the start of Run I and at the end of Run III, the end of Run III is found to deviate more from the unity. This deviation is taken as the systematic error, which is $0.55 \%$. At the Far Detector, an increasing in time linear trend is visible only after the photomultipliers retuning. Thus, the two-parameter straight line fit has been performed only after March 2006. The difference between the value of the fit at the end of Run III and the unity is used as the systematic error, which is $0.21 \%$. 


\subsection{The photomultiplier gains and the scintillator light emission}

As already explained, the overall detector response is a combination of the photomultiplier gain, of the scintillation light emission and of the electronics. The effect of the electronics can be considered negligible. Photomultiplier gains are measured through the light injection system and a comparison with the overall detector drift can be made. Figure 4.9 shows the percentage variation from the beam data taking period from May 2005 until June 2009 of the overall detector drift and the percentage variation of the photomultiplier gains, that have been measured independently. While the overall detector drift is decreasing over time, the gains are increasing due to ageing. Thus the scintillation light, that is not measured by any other dedicated system, must be decreasing over time more than the gains are increasing. As shown in figure 4.9 the photomultipliers gains are increasing by $\approx 2.5 \%$ a year at the Near Detector and by $\approx 2 \%$ a year at the Far Detector.

In a very simple model, the scintillation light emission can be derived by the overall response drift divided by the gains, as shown in figure 4.9. The behaviour at the Far Detector is easier to interpret as the temperature variation is small, allowing simple interpretation that the ageing is manifesting itself as a linear increase/decrease of the quantities. At the Near Detector this easy interpretation is not valid as the temperature variation is quite large and modifies the linear trend. The scintillator light emission seems to be decreasing by $\approx-3.5 \%$ a year at the Near Detector and by $\approx-3 \%$ a year at the Far Detector. As the scintillator light is decreasing more than the gains, the overall detector drift is decreasing.

\subsection{Temperature dependences}

Figures 4.10 and 4.11 show the detector responses over time and the relative hall temperature measurement over time. It is possible to evaluate the drift response change due to a temperature variation. As ageing does not affect the response on a scale of a few days, 

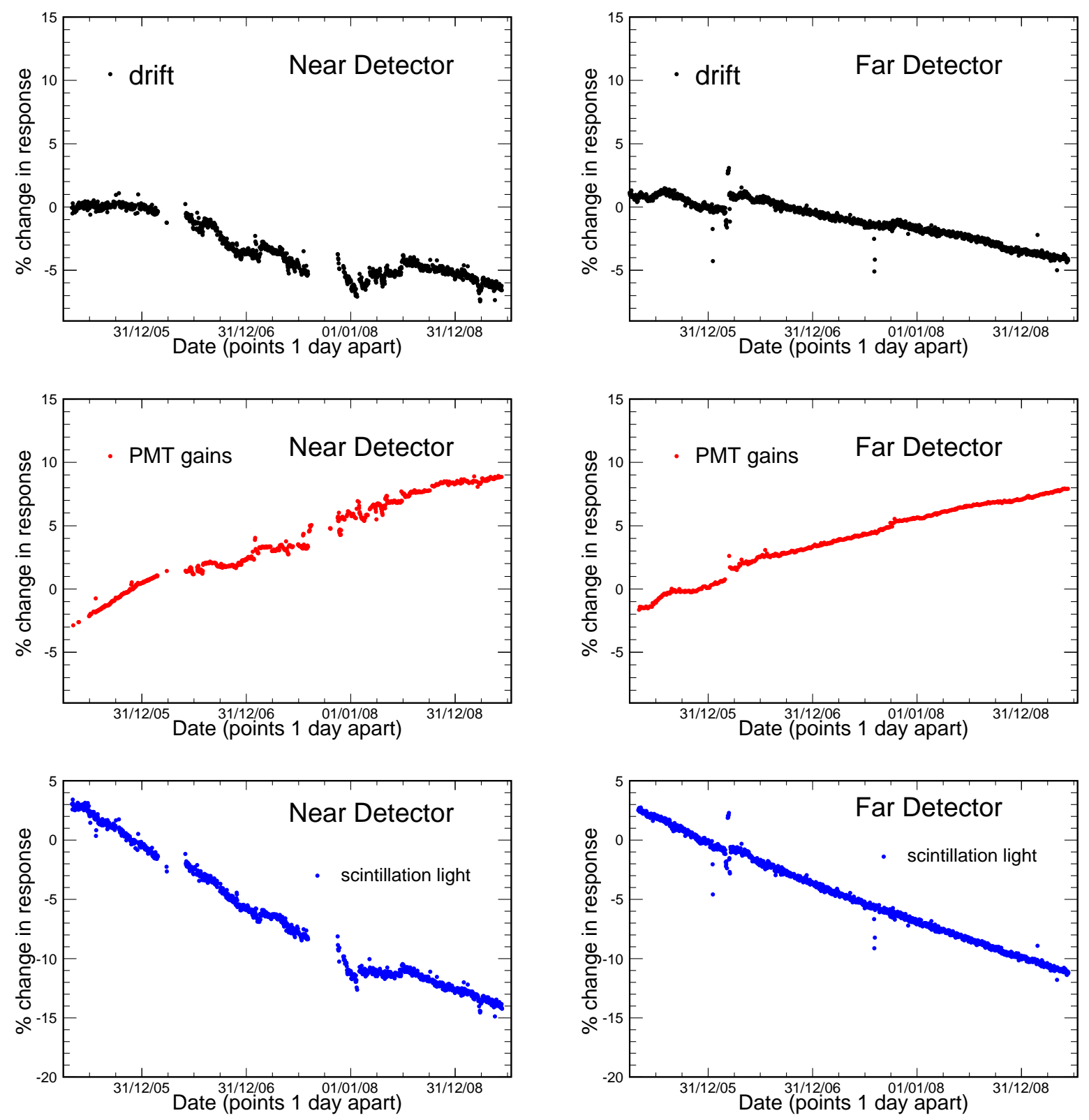

Figure 4.9: The plots show the behaviour over time of the overall detector response, of the photomultiplier gains (that are measured independently) and of the scintillation light emission (that can be derived from the other two quantities) for both the detectors. 
it is possible to consider the temperature variation as the cause of day-to-day changes in the detector drift. To avoid correlations and allow a bigger temperature variation, the detector response and temperature have been compared to the ones recorded three days earlier. Allowing a straight line fit to the percentage variation in detector response versus the temperature variation, the gradient of the returned straight line fit corresponds to the temperature dependence. Figure 4.12 shows the percentage variation in response and the variation in temperature. Superimposed the fit to a straight line is shown, as well as the value of the fitted gradient, intercept and the $\chi^{2} /$ degrees of freedom. The variation is consistent with a variation of $\approx-1.6 \mathrm{ADC} /{ }^{\circ} \mathrm{C}$ at the Near Detector and $\approx-3.5 \mathrm{ADC} /{ }^{\circ} \mathrm{C}$ at the Far Detector. The model seems to obtain good results at the Far Detector, while the Near Detector is poorly modelled. Considerations are given below. The same technique applied to the detector response has also been applied to the photomultiplier gains and scintillation light. Table 4.1 shows the fitted value of the dependence of the detector response, the photomultiplier gains and the light emission to the temperature. The variation in the photomultiplier gains is $\approx-0.22(\mathrm{pe} / \mathrm{ADC}) /{ }^{\circ} \mathrm{C}$ at the Near Detector and $\approx-0.07(\mathrm{pe} / \mathrm{ADC}) /{ }^{\circ} \mathrm{C}$ at the Far Detector. As expected from test-stand measurements the Near Detector scintillator light is less influenced by temperature changes than at the Far Detector. At the Far Detector the gains are almost insensitive to temperature variations and the whole temperature changes affect the scintillation light. At the Near Detector the temperature influences both gains and scintillation light equally. While at the Far Detector the hall temperature is a good indicator of the overall temperature, at the Near Detector the electronics are independently water cooled. Photomultipliers are expected to be affected by the temperature of the electronic racks and no effect of the electronics has been taken into account in this simple technique. For those reasons the simple description of the temperature dependence is only valid for the Far Detector, while for the Near Detector a more complex study should be performed to disentangle the independent effects of light and gain variations. 

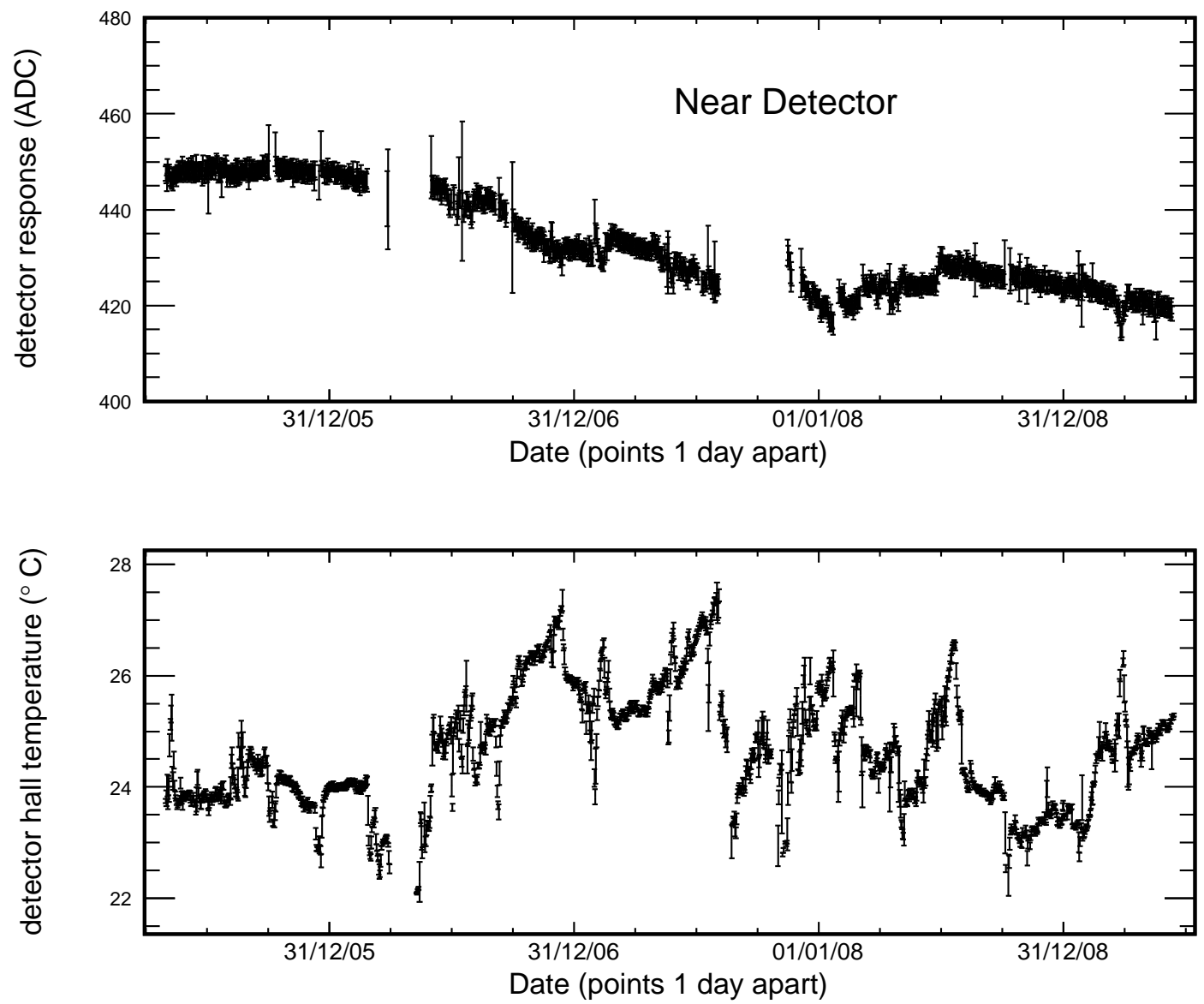

Figure 4.10: The Near Detector response drift over time (top) and the hall temperature over the time (bottom). The detector response has a long term drift behaviour which is due to ageing and a short term behaviour which is anticorrelated to the temperature.

\begin{tabular}{|l|l|l|}
\hline temperature effect on & $\mathrm{ND}$ variation $\left(\% /{ }^{\circ} \mathrm{C}\right)$ & $\mathrm{FD}$ variation $\left(\% /{ }^{\circ} \mathrm{C}\right)$ \\
\hline \hline drift constants & $-0.37 \pm 0.03$ & $-0.75 \pm 0.03$ \\
\hline photomultiplier gains & $-0.18 \pm 0.01$ & $-0.09 \pm 0.02$ \\
\hline scintillation light & $-0.17 \pm 0.03$ & $-0.61 \pm 0.04$ \\
\hline
\end{tabular}

Table 4.1: The measured temperature dependence for the detector drift, photomultiplier gains and scintillation light is reported for the Near Detector and the Far Detector. 

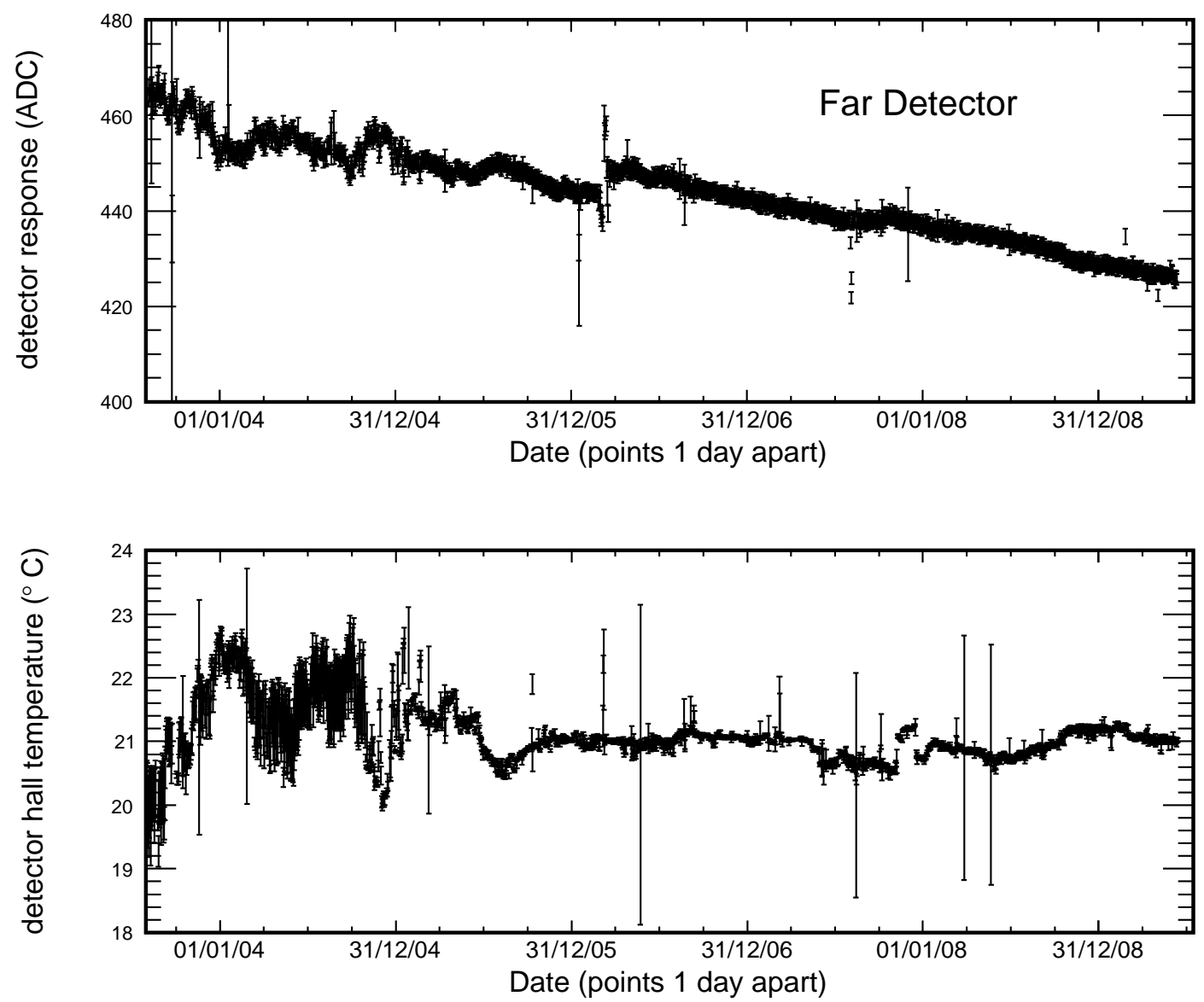

Figure 4.11: The Far Detector response drift over time (top) and the hall temperature over the time (bottom). The detector response has a long term drift behaviour which is due to ageing and a short term behaviour which is anticorrelated to the temperature. 

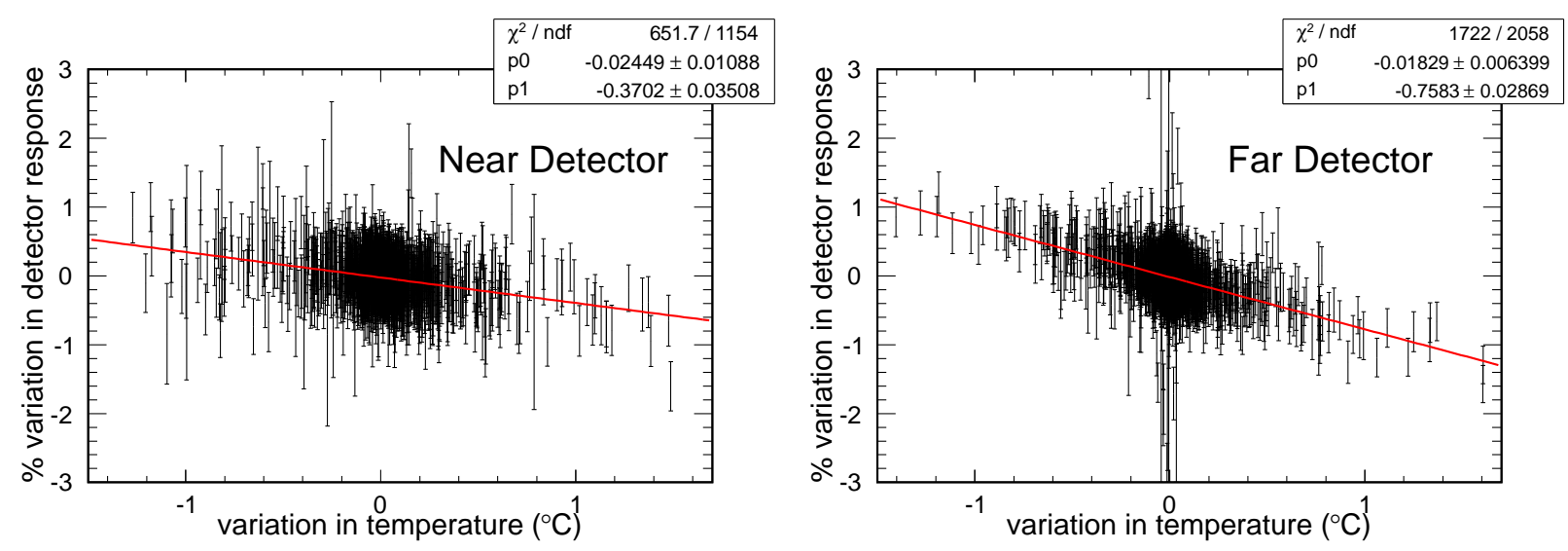

Figure 4.12: The percentage variation between drift constant and the one three day before versus the corresponding temperature variation. The Near Detector data on the left, the Far Detector data on the right. The red line corresponds to a straight line fit. The parameter $p 1$ represents the value of the gradient returned by the fit. The value of the $\chi^{2} /$ degrees of freedom in the fit is shown as well.

\subsection{Summary}

The MINOS detector calibration chain has been described in this chapter. Detector calibration is important in order to improve the resolution on the neutrino energy and ensure that the Near Detector and Far Detector are calibrated to the same energy. The latter aspect is fundamental in the oscillation analysis as the Near Detector data are used to predict the Far Detector spectrum. After the full calibration, the uncertainty on the relative calibration of the two detectors is $2.1 \%$, while the uncertainty due to calibration on the absolute shower energy scale is $5.7 \%$.

A step in the calibration, the drift correction, has been evaluated in this thesis: the median pulse height per plane deposited by cosmic muons in a day has been used to track the dependence of the detector response over time. This technique allows us to monitor the scintillator light level, the photomultiplier gains and the electronics stability. The Far Detector response linearly drifts downwards by about 10\% in 5 years and the Near Detector has drifted by $8 \%$ in 4 years. The detector response is the combination of the effects of the scintillator light level (which decreases at a rate of approximately $3 \% /$ year) and the photomultiplier gains (which increase at a rate of approximately $2 \% /$ year). During the 
first year of running the overall response of the Near Detector was flat as the light and gain effects cancel out. The systematic error associated to the drift calibration is $0.21 \%$ at the Far Detector and $0.55 \%$ at the Near Detector.

The daily drift corrections remove temperature dependent effects: the overall detector response is inversely correlated to the temperature. The Near Detector response varies by $-1.6 \mathrm{ADC} /{ }^{\circ} \mathrm{C}$, while the Far Detector one by $-3.5 \mathrm{ADC} /{ }^{\circ} \mathrm{C}$. In addition, a study of the separate dependence of the scintillator light and photomultiplier gains on the temperature has been performed. 


\section{Chapter 5}

\section{The NA49 Experiment}

This chapter describes the NA49 experiment physics goals and setup. NA49 has collected data on proton-carbon $(\mathrm{pC})$ interactions at a proton beam momentum of $158 \mathrm{GeV} / c$. Kaon production cross sections have been extracted in this thesis. Kaon production information evaluated here are important as a cross-check of the expected kaon production in the NuMI beamline for MINOS. Chapter 6 reports the kaon production results and chapter 7 describes how the NA49 results constrain the neutrino flux in MINOS.

\section{$5.1 \quad$ The experiment}

The NA49 experiment is a large acceptance detector that was located at the North Ex-

perimental area of the Super Proton Synchrotron (SPS) accelerator at the European Organisation for Nuclear Research (CERN). The main motivation of the experiment was the study of high energy nucleus nucleus collisions $(A+A)$ since at the energy of the SPS (158 GeV/nucleon) the transition of the hadronic matter to a deconfined state, known as Quark Gluon Plasma, is expected [115]. The majority of hadronic interactions occurring in nature happen at low squared momentum transferred $Q^{2}$, where the strong coupling constant $\alpha_{s}$ is large and the theory is not calculable perturbatively. This kind of interaction is often referred to as a "soft interaction". The NA49 experiment is placed in this context 


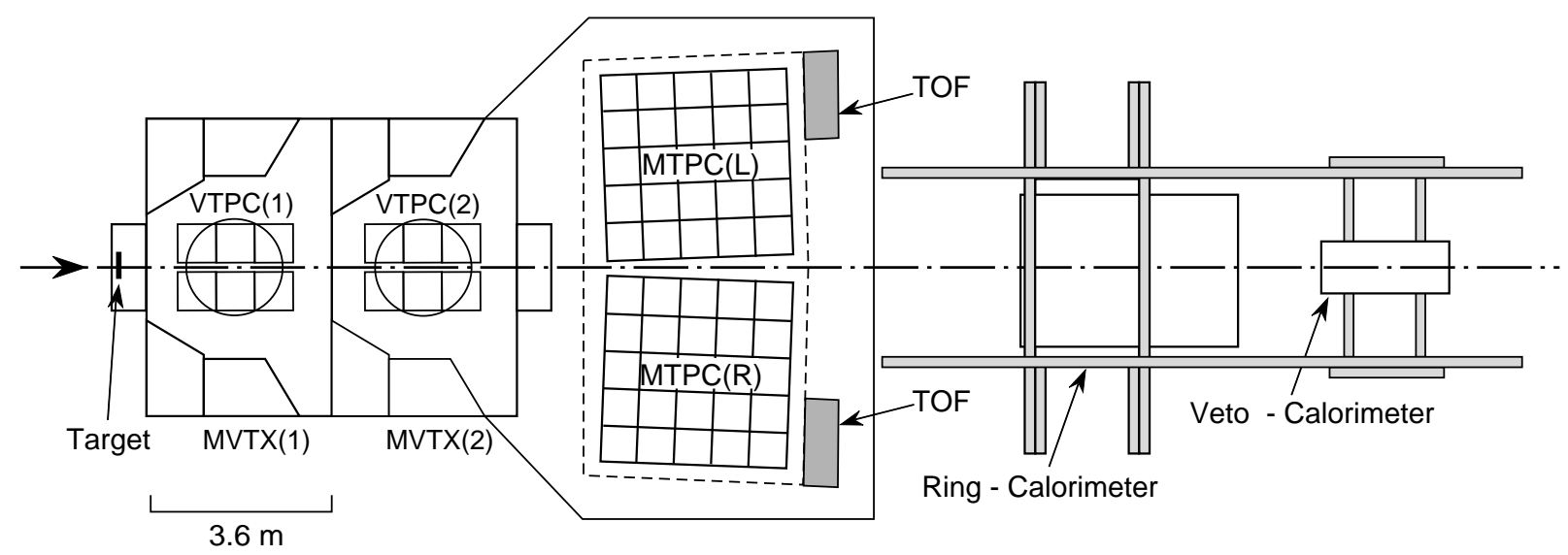

Fig. 1: NA 49 Experimental Layout

Figure 5.1: Following the beam direction, the figure shows a layout of the target, the vertex magnets, the four TPCs and the other auxiliary detectors. Details are given in the text.

and its aim is also to provide a consistent particle production dataset in proton-proton (pp), proton-nucleus ( $\mathrm{pC}$ in this specific case) and nucleus-nucleus ( $\mathrm{Pb}+\mathrm{Pb}$ in this specific case) interactions. The interpretation of hadron production data, which will not be analysed extensively in this thesis, still relies on phenomenological models that assume different hypotheses for the underlying partonic interactions. In this chapter the NA49 detector is described, with emphasis on those components that are relevant to the measurement presented in chapter 6 . Motivated by the expected high multiplicity of hadrons in the final state and the requirements for high acceptance coverage and particle identification, the NA49 is a magnet spectrometer with a set of Time Projection Chambers (TPCs) as main detectors. The layout of the experiment is shown in figure 5.1 viewed from above. The beam enters from the left of figure 5.1 and, after passing through beam counters, hits a target. The outgoing particles are deflected by two Vertex Magnets $(\operatorname{MVTX}(1)$ and MVTX $(2))$. The particle trajectories are recorded in four TPCs: two Vertex-TPCs (VTPC(1) and VTPC(2)) and two Main TPCs (MTPC(L) and MTPC(R)). There are other detectors which are part of the experiment but are not used in the pC analysis. The Time-of-Flight (ToF) detectors, which are placed at the end of the TPC system, are pixel or strip scintillating walls with a time resolution around 100 ps. Due to calibration problem at low multiplicity and 
the slow readout performances, the ToF detectors are not used in pp and pC data taking. The forward particles or fragments in $\mathrm{Pb}+\mathrm{Pb}$ collisions are detected in the Veto Chambers (VPC) and the calorimeters (Ring Calorimeter and Veto Calorimeter).

The centre of the NA49 coordinate system is in the middle of $\operatorname{VTPC}(2)$, with the $\mathrm{z}$ axis pointing downstream in the beam direction (the target is at about at $\mathrm{z}=-581 \mathrm{~cm}$ ). The positive $\mathrm{x}$ direction points horizontally to the left (upwards in figure 5.1, towards the side of the $\operatorname{MTPC}(\mathrm{L}))$.

\subsection{Beam detectors and interaction trigger}

Protons from the SPS with $400 \mathrm{GeV} / c$ momentum interact with a $10 \mathrm{~cm}$ long beryllium target in order to create a secondary beam by selecting only $158 \mathrm{GeV} / c$ momentum particles. The beam momentum resolution is $0.13 \%$. This secondary beam contains roughly 65\% protons which are identified with a CEDAR Ring Cerenkov counter. A set of upstream scintillators and Beam Position Detectors provide precise timing, charge and position measurement before the proton beam interacts with the target. The SPS provides a beam spill of $2-5$ s every $14-20 \mathrm{~s}$. Two scintillator counters S1 and S2 positioned along the $z$-axis upstream of the target provide timing and beam definition. In addition, a ring-shaped counter V0 vetoes the background from upstream interactions. The beam is defined as the coincidence between $\mathrm{S} 1, \mathrm{~S} 2$ and CEDAR, vetoed by the V0 activity. In pp and pC interactions there is much interest towards minimum bias events, i.e. non-diffracting events at very low transverse momentum. The TPC detectors do not cover the full forward region, but there is a gap to allow the beam that has not undergone interactions. In this gap, a scintillator of $2 \mathrm{~cm}$ diameter, called $\mathrm{S} 4$, is placed $4 \mathrm{~m}$ downstream of the target, between the vertex TPCs. The trigger condition is the requirement of a valid incoming beam in anti-coincidence to the activity of the $\mathrm{S} 4$ counter. The transverse position of the beam is measured by three small proportional chambers with cathode strip readout. 


\begin{tabular}{|l|l|l|}
\hline target & proton & carbon \\
\hline length $[\mathrm{cm}]$ & 20.29 & 0.7 \\
\hline diameter $[\mathrm{cm}]$ & 3 & 0.6 \\
\hline density $\left[\mathrm{g} / \mathrm{cm}^{3}\right]$ & 0.0707 & 1.83 \\
\hline atomic mass $[\mathrm{g} / \mathrm{mol}]$ & 1.0079 & 12.0107 \\
\hline target interaction length $[\%]$ & 2.8 & 1.5 \\
\hline
\end{tabular}

Table 5.1: The table shows the specifications of the $\mathrm{H}$ and $\mathrm{C}$ targets. The table is taken from [116].

\subsection{Targets}

A liquid hydrogen target (contained in a cylindrical vessel) is used in pp interactions while a graphite cylindrical target is employed in pC collisions. Table 5.1 shows some of the major characteristics of the adopted targets. The absolute cross section normalisation depends on the length and density of the targets. The length of the hydrogen target was determined offline by fitting the vertex distributions of high multiplicity empty targets events, giving a $0.25 \%$ precision in the measurement [117]. The carbon target was made industrially, with a precision of $1 \mu \mathrm{m}$ [117].

\subsection{The Magnets}

Two super-conducting dipole magnets are centred around the beam line. The field is $1.5 \mathrm{~T}$ in the first magnet and $1.1 \mathrm{~T}$ in the second one. The width of the magnets is $5.7 \mathrm{~m}$ and each has a length of $3.6 \mathrm{~m}$. The magnets are cooled by liquid helium. In order to operate the TPCs in such a strong magnetic field, a precise knowledge of the magnitude and orientation of the magnetic field is required. The magnetic field allows particle momentum measurement, with a resolution of the order of $\Delta p / p^{2} \sim 10^{-5}-10^{-4}(\mathrm{GeV} / c)^{-1}$.

\subsection{The Time Projection Chamber system}

Particles produced in the interaction between the beam and the target are detected in the TPCs. The two Vertex TPCs are placed inside the aperture of the magnets, allowing track 
momentum measurement from curvature. The two Main TPCs are outside the magnetic field. As visible from figure 5.1, the detectors are arranged in a symmetrical fashion along the beam axis. The gap in the detector volume is required in the case of heavy ion collisions, as the space charge effects in the wire region due to the beam detection would be excessive. To supply coverage to this gap, a small TPC, called the Gap TPC (GTPC) has been introduced for running with the proton beam. This extra detector recovers part of the missing acceptance.

TPCs consist of two parts: a sensitive volume (inside a field cage) and the readout chamber. Tracks exiting from the target interact in the sensitive volume of the TPC, which is filled with gas, and ionise electrons in the gas along their trajectory. An electric field of order of $\approx 150-200 \mathrm{~V} / \mathrm{cm}$ is applied to the sensitive volume to allow the electrons from the ionisation drift to reach the readout chamber (on the top of the TPCs in the case of NA49). To assure uniformity in the applied electric field in the whole TPCs volume, a field cage of aluminized Mylar strips of $25 \mu \mathrm{m}$ thickness and $\approx 1.3 \mathrm{~cm}$ width is used. The high voltage planes are made of the same type of strips. The field cage is insulated by a gas envelope made of double layer $125 \mu \mathrm{m}$ Mylar foil glued to a double frame system of $6 \mathrm{~mm}$ thick fibreglass. The volume between the layers is filled with nitrogen in order to minimise gas impurity diffusion. Figure 5.2 shows a schematic picture of the details of the readout system. Electrons drifting from the primary ionisation encounter a gating grid, whose purpose is to stop the electrons when there is no trigger and to prevent ions produced in the readout process to enter the TPC sensitive volume. Electrons pass the cathode plane (also referred as the Frisch grid) which is at ground potential. Electrons then arrive at the sense wires, which are at high potential $(+1200 \mathrm{~V})$, and an ionising avalanche is created with typical gain of $10^{3}-10^{4}$. An electric pulse is induced on the back of the pads that constitute the cathode plane. The segmentation of the readout in pads gives information about two of the space coordinates, while the drift time of the electrons allows reconstruction of the third spatial coordinate (vertical) of the track trajectory. The pad dimensions vary according to the TPC, with higher granularity in VTPC1 and smaller 


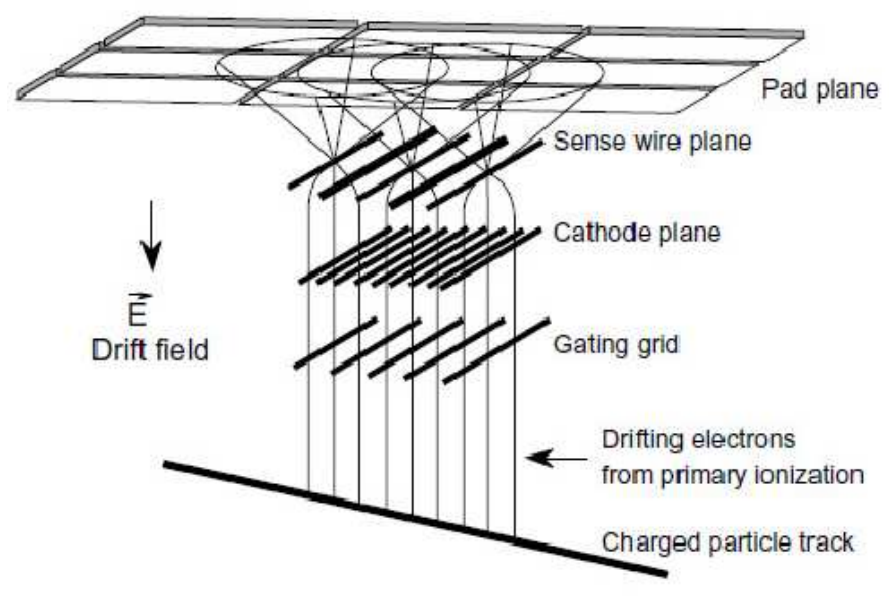

Figure 5.2: The figure shows a schematic diagram of the readout of the TPCs. The amplification stage of the electrons coming from the ionisation of the track happens in proximity of the sense wires and a signal is induced on segmented pads. Figure taken from [116].

\begin{tabular}{|l|l|l|l|}
\hline Dimensions (m) & VTPC-1,2 & MTPC-L/R & GTPC \\
\hline Width & 2 & 3.9 & 0.815 \\
\hline Length & 2.5 & 3.9 & 0.30 \\
\hline Height & 0.98 & 1.80 & 0.70 \\
\hline Drift length & 0.666 & 1.117 & 0.59 \\
\hline
\end{tabular}

Table 5.2: The table shows the geometrical dimensions of the NA49 TPC system. The table is taken from [116].

in the MTPCs. The smaller pad size is $16 \times 3.5 \mathrm{~mm}^{2}$ and the biggest is $40 \times 5.5 \mathrm{~mm}^{2}$. Tracks are measured with a precision of a few hundreds $\mu \mathrm{m}$.

Table 5.2 shows the dimensions and drift characteristics of the TPCs. The TPCs sensitive volume is filled with a gas mixture: $\mathrm{Ne} / \mathrm{CO}_{2}(90 / 10)$ for the VTPCs and GTPC and $\mathrm{Ar} / \mathrm{CH}_{4} / \mathrm{CO}_{2}(90 / 5 / 5)$ for the MTPCs. The gas mixtures have been chosen taking into account the low transverse and longitudinal diffusion coefficients and the drift velocities. More details about the choice are given in [116]. The $\mathrm{CO}_{2}$ in the gas suffers from electron attachment, so the quantity has to be limited. Nevertheless, $\mathrm{CO}_{2}$ is used to limit the diffusion. The gas purity and composition is controlled by a gas system. The temperature is stabilised with a precision of $0.1^{\circ} \mathrm{C}$.

Calibration of the electronics and gas gain has been achieved using a radioactive source of ${ }^{83} \mathrm{Kr}$ gas added to the normal gas mixture. This technique also provided time dependence 
corrections for the different data taking runs. Gas pressure is the main aspect sensitive to time difference. The time dependence of the particle energy loss has been studied and corrected by looking at the mean of charge clusters in each readout module. More details about the TPCs response calibration are given in section 6.1.1.

\subsection{Electronics and data acquisition}

The readout system has to collect the signal from 182000 pads. The front-end cards are mounted directly on the readout chamber to minimises noise and the signal is amplified and shaped before being digitised. For each pad, the time structure of the signal is sampled in 512 time buckets (sampling at a frequency of $10 \mathrm{MHz}$ ), in order to keep timing information that needs to be used to reconstruct the vertical position along the track. The digitisation performs zero suppression and noise rejection, allowing a reduction of data from $100 \mathrm{MB}$ to 1.5 MB for a single event. The data is transferred on Raw Data Tapes. The data acquisition is optimised for the SPS spill of $2.4-4 \mathrm{~s}$ in $14-16 \mathrm{~s}$ cycles.

\subsection{Event reconstruction}

Figure 5.3 shows an event display for an high multiplicity collision in $\mathrm{Pb}+\mathrm{Pb}$ interactions.

The main steps of the event reconstruction, which is described in detail in $[118,116]$, are listed below:

- The ionisation induced along the track is collected in charge clusters. The position of each cluster is determined by the centre of the charge distribution. If the electric field and the magnetic field are not perfectly parallel in the VTPCs, the paths of the drifting electrons are no longer vertical lines but they are distorted. In addition, the inhomogeneity of the electric field can cause electron distortion in both the MTPCs and VTPCs. A MC-based correction is applied at this stage to remove distortion effects. 


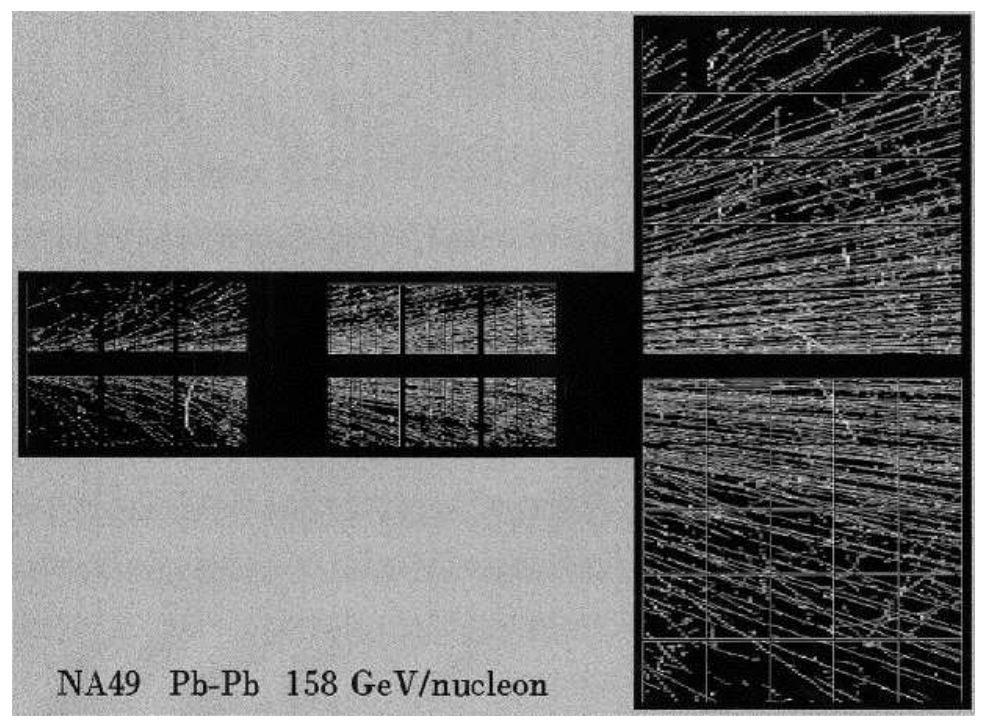

Figure 5.3: The figure shows an event display for a high multiplicity collision in $\mathrm{Pb}+\mathrm{Pb}$ interactions. The figure is taken from [116].

- The charge clusters are matched to local tracks in each TPC separately.

- Global tracks are formed from the union of the different track segments.

- The main vertex is found by an iterative algorithm, by sequentially removing outlying tracks. Low multiplicity events, that are events with a small number of tracks have a lower longitudinal vertex resolution.

- Although most of the tracks come from the primary interaction vertex in the target, some strange neutral particles $\left(K_{s}\right.$ and $\left.\Lambda_{s}\right)$ decay weakly later, giving the possibility to have tracks starting from a secondary vertex $V^{0}$. A specific secondary vertex algorithm is used to identify secondary tracks.

- The trajectory of tracks originated from the main vertex is fitted in the mapped magnetic field, providing momentum determination. 


\subsection{Data analysed}

The data analysed in this thesis are a small sample of proton-carbon minimum bias collisions (approximately 377 thousands inelastic events) at $158 \mathrm{GeV} / c$ proton beam momentum. As described in chapter 6 the extraction of kaon inclusive production cross section is performed through a fit to the specific ionisation, which is a function of the particle mass for particles at the same momentum. To prove the validity of the method, a cross check of the fitting technique has been performed on a high statistics sample of proton-proton minimum bias collisions (approximately 4.8 million inelastic events) at the same beam momentum. Ideally, as the extraction of the kaon cross section has been performed on this data set, it would be worth investigating the differences in hadron production between the two samples. Since the projectile is the same in the two cases (the proton beam), the difference in physics is described by the different target (either p or $C$ ). Due to the restricted number of nucleons in the target, the inelastic cross section in pp collisions is expected to be smaller than the corresponding cross section in pC collisions (approximatively $32 \mathrm{mb}$ versus $226 \mathrm{mb}$ ). At the same time, while pp collisions undergo single projectile collisions, $\mathrm{pC}$ collisions have an average of $\approx 1.7$ collisions [117]. 


\section{Chapter 6}

\section{Inclusive invariant kaon production cross section in $\mathrm{pC}$ interactions}

Neutrino flux uncertainties in MINOS and other experiments which produce a man-made beam are mainly due to large uncertainties in the secondary particles produced off the target from the primary beam. Monte Carlo models for this kind of interaction suffer from uncertainties as big as 30-40\%. Some new data in conditions of interest for MINOS have been made available in recent years by the NA49 collaboration. Pion production inclusive invariant cross sections in pC interactions at $158 \mathrm{GeV} / c$ proton beam momentum have been published in $[119,120]$. Despite the different data taking conditions between MINOS and NA49 (the target thickness and the primary proton beam energy), the pion results from NA49 have been of great use in MINOS. In this thesis an analysis of the pC interaction dataset taken by NA49 in 2002 has been performed in order to obtain kaon production inclusive invariant cross section results. As a cross check sample, the pp interaction dataset, taken in three runs spanning from 1999 to 2002 has also been analysed. Given the years elapsed since the data taking, most of the detector modelling study, $d E / d x$ calibration, empty target reduction cuts and all the Monte Carlo studies had been performed by NA49 collaborators. The effort put in this thesis has been towards a different approach to the particle separation through a fitting to the $d E / d x$, with detailed 
studies of the sensitivity of the fits given the low statistics the pC dataset is suffering from. Errors on the fitted parameters have been extensively evaluated for the first time using $\Delta \chi^{2}$ projections. The data file type made available to the author by the NA49 collaborators were the so-called $\mu \mathrm{DST}$, files that retain information about the event multiplicity, single track momentum, mean energy loss and resolution and the number of clusters sampled along the track. The results presented in this thesis are not the NA49 collaboration official results, but consistency checks have been performed.

\subsection{Energy loss by ionisation and particle identifica- tion}

Charged particles travelling through a medium lose energy by ionising the electrons of the material they are travelling into. The slower the particle is, the more it ionises. The energy loss is given by the Bethe-Bloch equation [12]:

$$
\frac{d E}{d x}=4 \pi N_{A} r_{e}^{2} m_{e} c^{2} z^{2} \frac{Z}{A} \frac{1}{\beta^{2}}\left[\frac{1}{2} \ln \frac{2 m_{e} c^{2} \beta^{2} \gamma^{2} T_{\max }}{I^{2}}-\beta^{2}-\frac{\delta(\beta \gamma)}{2}\right]
$$

where $Z$ and $A$ are the atomic mass and the atomic number of the medium, $z$ is the charge of the particle in units of electron charge, $I$ is the mean ionisation energy $(\approx 10 \mathrm{eV} \times Z), \beta$ is defined as $v / c, T_{\max }$ is the maximum kinetic energy that can be transferred to an electron in a single collision, $\delta(\beta \gamma)$ is the density effect correction. At low momentum, shortly before the particle stops, the ionisation is at the maximum because it has more time to perturb the electrons of the medium, as described by the $1 / \beta^{2}$ term in equation (6.1). When the particle is still fast, it ionises less, as it is described by the $\ln \left(\beta^{2}\right)$ dependence. Between the two regimes, there is a point in which the two trends balance out and the particle reaches the minimum ionising stage. Figure 6.1 shows the Bethe-Bloch curve for a $\mu^{+}$in copper. The different regimes for the ionisation are visible. The range of momentum of interest to NA49 is up to $100 \mathrm{GeV}$, i. e. before the radiative losses become important. 


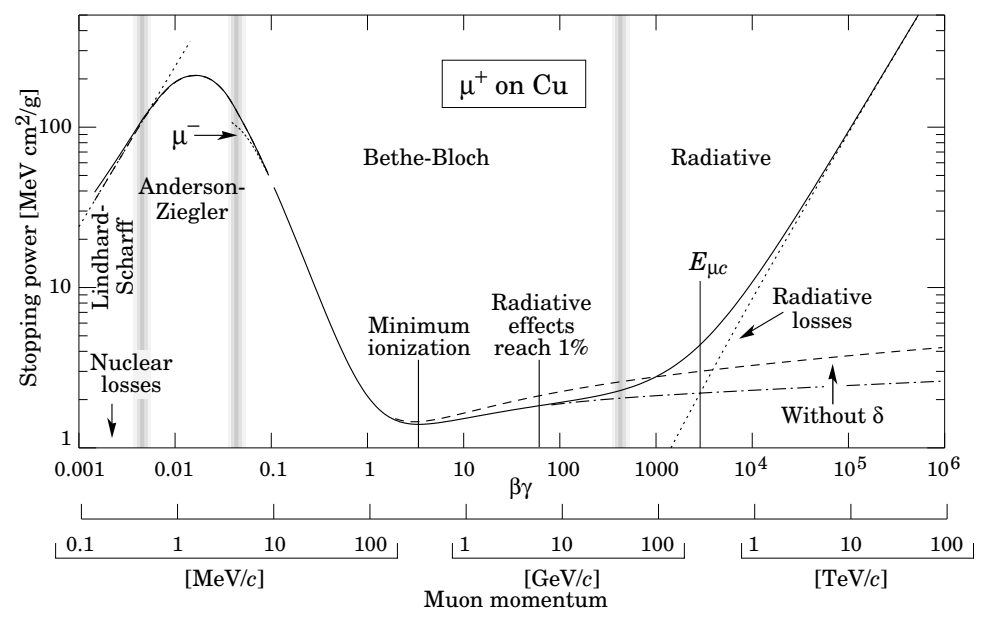

Figure 6.1: The figure shows the stopping power (the opposite sign of the energy loss) for a positive muon in copper as a function of $\beta \gamma(=p / M C)$. Figure taken from [12].

To first approximation, the Bethe-Bloch equation is a function only of the particle velocity $\beta / c$, this means that all particles that travel at the same velocity will loose the same amount of energy. By selecting particles that have the same momentum but different mass, the Bethe-Bloch equation can be used to identify particles. A parameterisation for the expected energy loss by ionisation in the NA49 TPCs has been developed in [121], taking into account the different gas medium for the VTPCs and MTPCs. Particle momenta in NA49 can be measured from the curvature measurements (tracks are typically long and do not stop in the detector). The estimate of the energy deposited by a particle is calculated from a $50 \%$ truncated mean of the clusters with lower energy. The optimization of the truncation value for the NA49 detectors has been studied in [121] and it is justified by the nature of the energy loss in collisions that follows a Landau distribution, where the long tail in the ionization is given by the fluctuations in the ionization. Figure 6.2 shows the energy loss as measured in the NA49 detector for data. Superimposed are the expected energy loss for pions, kaons, protons and electrons as parameterised in [121]. The region below approximately $4 \mathrm{GeV}$ in momentum is referred to as the "cross-over region", as the $d E / d x$ of the different particles cross each other. The kaon $d E / d x$ is above the proton one for momentum greater than approximately $2.5 \mathrm{GeV} / c$ and below the pion $d E / d x$ for momentum above $1 \mathrm{GeV} / c$. Particle identification in the cross-over region has not been 


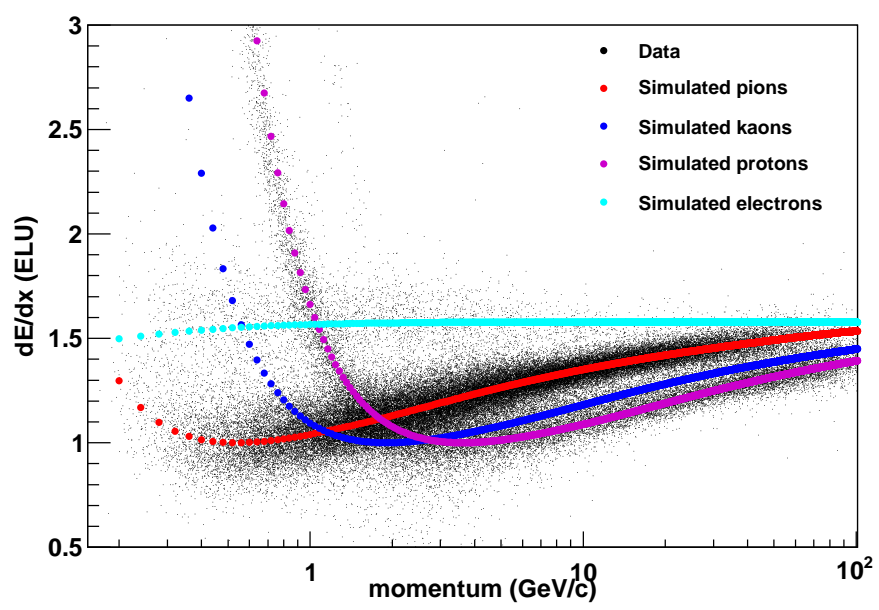

Figure 6.2: The figure shows the measured energy deposited in the NA49 TPCs as a function of momentum. Superimposed to the data are the ideal predicted $d E / d x$ for pions (in red), kaons (in blue), protons (in purple) and electron (in light blue).

performed given the low statistics available. By convention, it is common to refer to the units of the energy loss as $\mathrm{ELU}\left(\mathrm{MeV} \mathrm{cm}^{2} / \mathrm{g}\right)$.

\subsection{1 $d E / d x$ calibration steps}

As explained in section 5.5 the first step in the calibration of the TPC signals is obtained from the radioactive decays of ${ }^{83} \mathrm{Kr}$ that is injected into the gas system. The charge clusters produced by the ${ }^{83} \mathrm{Kr}$ have a higher energy than the expected energy deposition for a minimum ionising particle, so this method provides relative channel calibration. Calibration runs were taken before and after each SPS running period of 6-8 weeks. The gas gain is strongly dependent on the gas pressure and temperature, which can cause daily variations during the run period. Environmental settings were constantly monitored and possible time dependent gas gain variations have been corrected for. Other corrections, which have been separately evaluated, need to be applied. The charge collected at the pad plane depends on the relative angle between the track and the pad. Pads are generally oriented to favour the expected track orientations, but a geometrical correction has to be applied. The charge cluster is also affected by the long drift length travelled by electrons to reach the readout 
chamber. A small part of the charge is loss due to electron attachment, which has an effect up to $2 \%$ in the MTPCs and $4 \%$ in the VTPCs. A bigger effect is given by the fact that the electronics applies a 5 ADC zero suppression thresold and the charge cluster distribution widens due to diffusion. This combination of effects creates a charge loss that can reach a $7 \%$ deficit per metre drift length. Monte Carlo corrections have been studied in order to cancel these effects.

\subsection{Event and track selection}

A series of cuts are applied on whole events and single tracks in order to perform the track selection:

- To properly extract the resulting hadronization from only the target material, the standard technique is to collect both full and empty target data, in order to subtract the normalised contributions from the empty target data, i.e. hadronization from the material surrounding the target. The NA49 collaborators have developed a series of cuts on the Beam Position Detectors profile and on the $z$ position of the fitted track vertex to reduce the empty target contribution to particle yields: a detailed description of the problem and the vertex cut values are available in [122]. The series of vertex cuts reduce the interaction from non-target material from $30 \%$ to $16 \%$ in $\mathrm{pC}$ interactions. It needs to be noted that the cuts on Beam Position Detectors do not introduce any bias in the sample, as they are made before the interaction with the target. Cuts on the $z$ position of the fitted vertex could in principle introduce a bias in the events selected: this will be treated in section 6.10 .

- Tracks are selected according to their charge sign. The particle charge is obtained from the curvature of the fitted tracks in the magnetic field [123].

- Tracks are successively separated according to their kinematic properties such as the longitudinal momentum, the transverse momentum and the azimuthal angle. A 
detailed description of this procedure is given in section 6.3 .

\subsection{Binning in $x_{F}, p_{T}$}

The differential production cross section is a function of momentum. The particle density in the longitudinal direction increases roughly linearly with increasing energy in the centre of mass $(\sqrt{s}=17.3 \mathrm{GeV}$ in NA49 pC interactions), whereas in the transverse direction the density increases in a rough exponential manner and its evolution with the interaction energy is very moderate. For these reasons, it has been decided to present results in terms of a longitudinal quantity $x_{F}$ and transverse momentum $p_{T}$. The $x_{F}$ variable, suggested by Feynman as a scaling variable for the invariant cross sections [124], is defined as the ratio of the longitudinal momentum in the centre of mass frame $p_{L}^{*}$ to half of the energy in the centre of mass $\sqrt{s} / 2$ :

$$
x_{F}=\frac{2 p_{L}^{*}}{\sqrt{s}}=\frac{2 \gamma\left(p_{L}-\beta E\right)}{\sqrt{s}} .
$$

The second equality represents the Lorentz transformation that expresses the longitudinal momentum in the centre of mass frame as a function of the longitudinal momentum in the laboratory frame $p_{L}$ and energy $E$. Looking at $x_{F}$ two points need to be noted: $x_{F}$ can be negative, to describe particles emitted backwards in the centre of mass frame, and the $x_{F}$ dependence on $E$ causes a dependence on the particle mass. A pion and a kaon having equal longitudinal and transverse momentum in the centre of mass frame will have different $x_{F}$. At higher energies the difference between masses become negligible: for practical use at $x_{F} \geq 0.1$, there is no difference between the pion or kaon mass assumption. In this thesis the kaon mass assumption is considered in the evaluation of $x_{F}$, unless differently stated. Figure 6.3 shows the binning scheme in $x_{F}$, assuming the kaon mass assumption, and $p_{T}$ for which results of the invariant kaon cross section will be given. Particle identification needs to be achieve in a range of momenta from 5 to $40 \mathrm{GeV}$. From the MINOS point of view the upper limit in kaon momentum translates to $\nu_{\mu}$ energies up to $25 \mathrm{GeV}$. Unfortunately this 


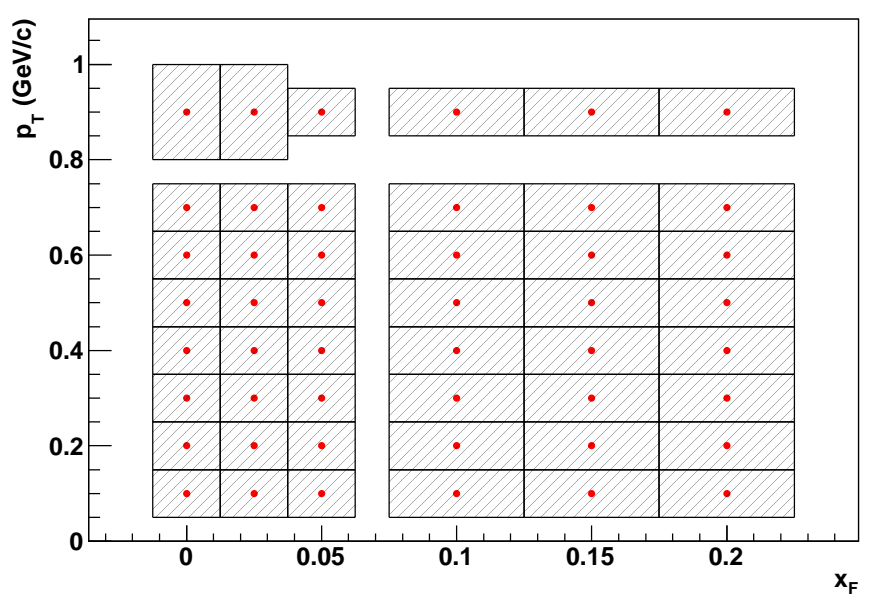

Figure 6.3: Binning scheme for the kaon identification. In the evaluation of $x_{F}$ the assumption of the kaon mass for all particles has been made.

region of neutrino energy is still dominated by the pion contribution: the kaon contribution becomes important around $30 \mathrm{GeV}$ in neutrino energy. The statistics at higher longitudinal and transverse momentum in NA49 starts to become limited, making the separation fit unstable.

Figure 6.4 shows the number of charge clusters sampled along each track. The spikes at 50 points represent tracks detected by a single VTPC, at 80 points represent tracks detected by the MTPC, at 150 points represent tracks detected by a VTPC and the MTPC. The maximum possible number of points detected is 234 . The number of points sampled influences the resolution on both the energy loss by ionisation and the momentum. The total momentum resolution is $d p / p^{2}=7.0 \times 10^{-4}(\mathrm{GeV} / c)^{-1}$ for tracks passing through VTPC-1 only $\left(0.5-8 \mathrm{GeV} / c\right.$ in momentum) and $d p / p^{2}=0.3 \times 10^{-4}(\mathrm{GeV} / c)^{-1}$ for tracks detected in VTPC-2 and one MTPC (4-100 GeV/c in momentum range) [116]. Provided enough charged samples can be collected along the track, low momentum particles have a better resolution because they are bent more by the magnetic field.

From the track momentum components in the transverse plane, it is possible to define the azimuthal angle $\phi$ as:

$$
\phi=\tan ^{-1} \frac{p_{y}}{p_{x}} .
$$




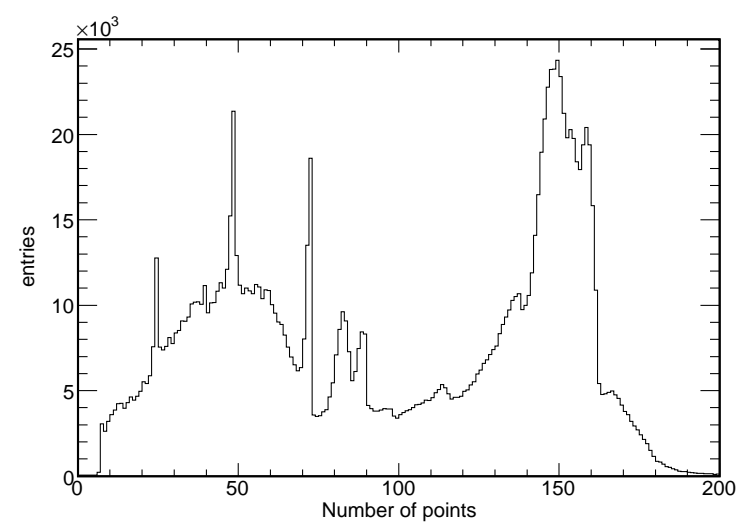

Figure 6.4: The figure shows the distribution of charge clusters sampled along each track in the NA49 TPCs.

Particle production in each $\left(x_{F}, p_{T}\right)$ bin is evaluated imposing a particular $\phi$ range in each bin, due to acceptance constraints. The yield for the full $2 \pi$ range can be restored by multiplying the results for the fraction of $\phi$ considered out of the full planar angle. As the number of points sampled along the track is a function of $\phi$, imposing a cut on the $\phi$ range is equivalent to requiring that tracks deposit a certain number of charge clusters in the detector. In particular the $\phi$ cut is designed to ensure that positive particles are detected in a particular region of the detector and negative ones in another region. These requirements are needed to improve the resolution of the energy loss as tracks will induce enough charge clusters in the detector and the readout pads will be in the optimal orientation for the tracks. Figure 6.5 shows the geometry for the $\phi$ cut. Positive tracks are selected in a range of angles centred at $\phi=0^{\circ}$, negative tracks are selected in a range of angles centred at $\phi=180^{\circ}$. Figure 6.6 shows the distribution of the points sampled along the track as a function of the azimuthal angle $\phi$ for charge selected tracks. Superimposed in red is the mean distribution for tracks that pass the $\phi$ cut requirements. Ideally, when the statistics allows it, the cut on $\phi$ has been chosen to guarantee the mean number of clusters along the track to be large and constant along the $\phi$ range. 


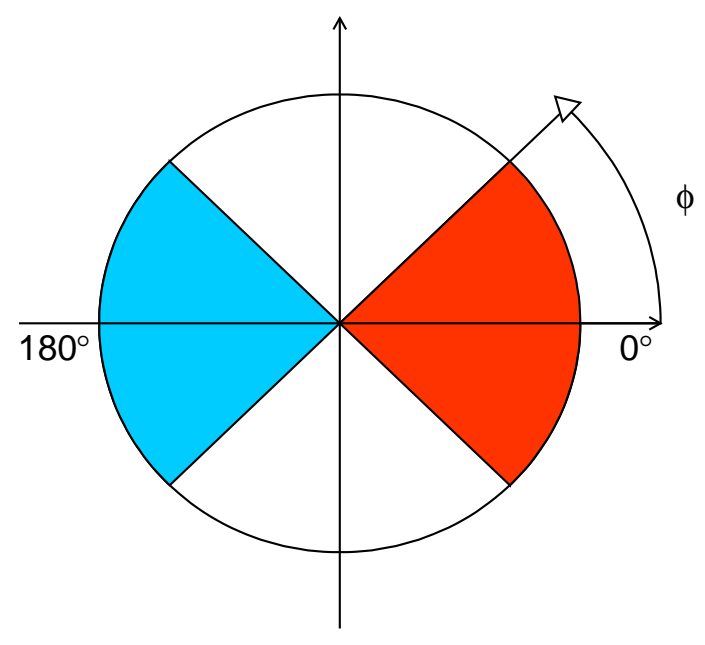

Figure 6.5: The figure shows the geometry for the azimuthal angle $\phi$. Positive charged tracks are selected only in a range around $\phi=0^{\circ}$, as shown by the orange segment. Negative charged tracks are selected in a range of angles centred at $\phi=180^{\circ}$, as shown by the light blue region. The position of the cut is tuned for each bin in $\left(x_{F}, p_{T}\right)$ in order to select tracks with a high average number of points sampled, if possible.

\subsection{Detector response and modelling}

A customised parameterisation of the specific ionisation in the NA49 TPCs has been extensively studied in [121] and [125]. As the MTPCs and the VTPCs have different gas mixtures, the truncated mean of the energy deposited in the charge clusters is studied separately in the two parts of the detector. A global $d E / d x$ is then obtained by a linear transformation of the $d E / d x$ in the VTPCs to the $d E / d x$ in the MTPCs.

Once the expected energy loss for each particle type is evaluated, resolution smearing has to be taken into account. The dependence of the ionisation resolution $\frac{\sigma}{d E / d x}$ (evaluated as the RMS divided by the truncated mean of the ionisation distribution) has been parameterised [121] as:

$$
\frac{\sigma}{d E / d x}=\sigma_{0} N_{p}^{\beta}(d E / d x)^{\alpha}
$$

where $\sigma_{0}$ represents the intrinsic resolution, evaluated by the fit to be 0.41 in the VTPCs and 0.35 in the MTPCs, and $N_{p}$ represents the number of sampled points along the track. The 

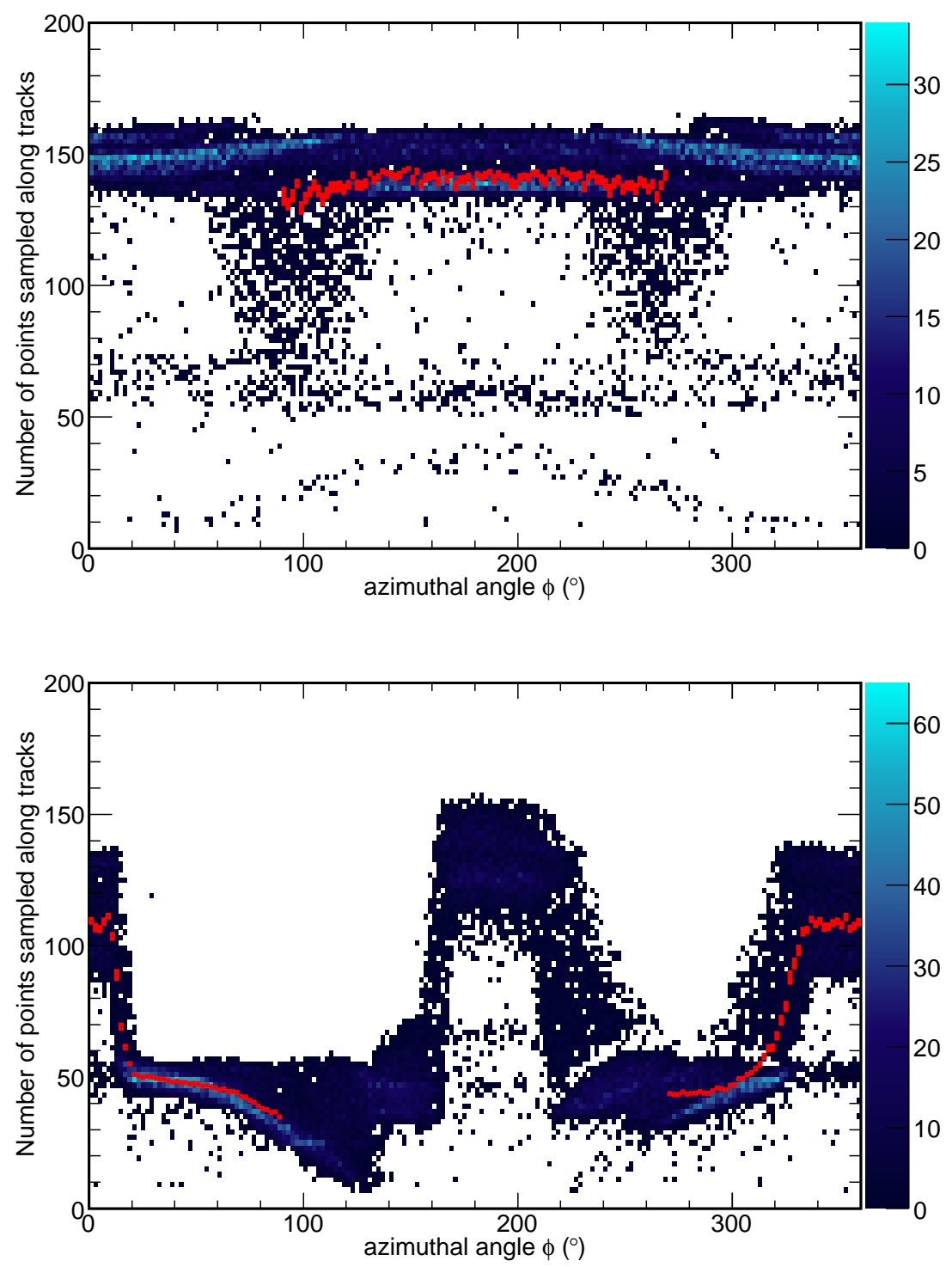

Figure 6.6: The figures show the number of clusters sampled along tracks as a function of $\phi$. Superimposed in red is the mean distribution for tracks passing the $\phi$ cut requirement for the particular bin. On the top is for selected negative tracks for $x_{F}=0.025$ and $p_{T}=0.2 \mathrm{GeV} / c$. The $\phi$ cut range accepted is $\left(90^{\circ}, 270^{\circ}\right)$. On the bottom the distribution is for positive selected tracks for $x_{F}=0.0$ and $p_{T}=0.3 \mathrm{GeV} / c$. The range of $\phi$ accepted is $\left(0^{\circ}, 95^{\circ}\right) \cup\left(265^{\circ}\right.$, $\left.360^{\circ}\right)$. 

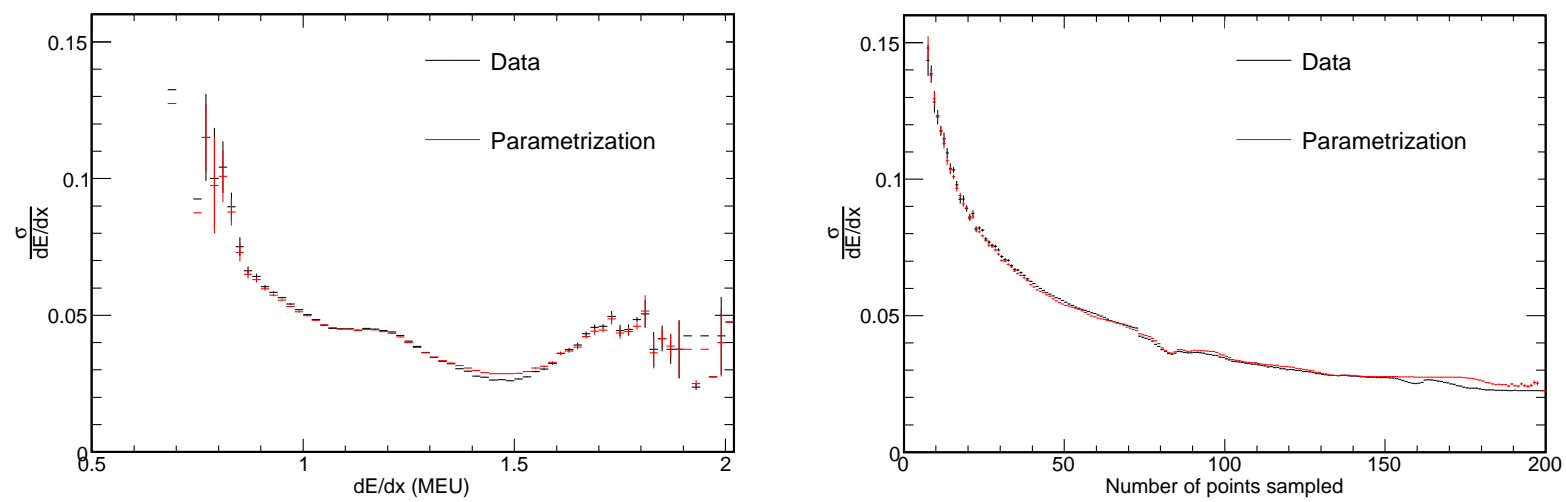

Figure 6.7: In the figure the resolution of the specific ionisation is presented as a function of the specific ionisation (on the left) and of the number of points sampled along the track (on the right). Black represents the measured values for data, red is the NA49 parameterisation proposed in equation (6.4).

values fitted for the parameters are $\beta=-0.50 \pm 0.01$ (as expected from Poisson statistics) and $\alpha=-0.39 \pm 0.03[121,125]$. Figure 6.7 shows the average resolution $\frac{\sigma}{d E / d x}$ as a function of the $d E / d x$ (left) and of the points sampled along the track (right) for the data and for the parameterisation proposed in [121]. The data points and the parameterisation agree remarkably well.

The NA49 data analysis does not use a full detector simulation in order to perform the fit to the specific ionisation to separate the particle types. Real data track information (the momentum, $d E / d x$, the resolution $\sigma$ and the number of points sampled along the track $N_{p}$ ) are used as an input to predict the $d E / d x$ distributions for the four particle types, while the relative normalisation is determined from a fit. For each real data track, the measured track momentum is used to determined the expected $(d E / d x)_{\text {exp }}$ for each particle type from the parameterisation of the specific ionisation in NA49. Assuming no dependence of the number of points and of the intrinsic resolution from the $d E / d x$, equation (6.4) is used to determine the expected resolution $\sigma_{\exp }$ following:

$$
\sigma_{\exp }=\sigma_{\text {data }}\left(\frac{(d E / d x)_{\exp }}{(d E / d x)_{\text {data }}}\right)^{\alpha+1}
$$

While the standard technique involves Gaussian distributions to construct the response 
in specific ionisation corresponding to each particle type, a data driven distortion relative to Gaussian distributions has been proposed in [125]. The distortion has been determined to have the form:

$$
g^{\prime}=g+Z\left(g^{2}-1\right)
$$

where $g$ is a variable from the standard normal distribution (mean of 0 and RMS of 1), $g^{\prime}$ follows the distorted distribution and $Z$ is the distortion factor. If $g \sigma+d E / d x$ is a value picked from the normal distribution with mean $d E / d x$ and width $\sigma$, then $g^{\prime} \sigma+d E / d x$ represents a value picked from the distorted Gaussian with the same mean but modified RMS. The distortion factor has been parameterised to be: $Z=0.57 \frac{\sigma}{d E / d x}$. The distortion changes the integral of the tail of the distribution by $\pm 1.5 \%$ (the tail has been defined by setting a cut at 1 RMS from the center of the distribution).

\subsection{Particle identification using a fit to the specific ionisation}

Once the bins in $\left(x_{F}, p_{T}\right)$ and $\phi$ are defined, the particle identification in each bin is obtained by fitting the sum of the four expected $d E / d x$ distributions to the data spectrum in order to perform particle identification. The integral of the entries in each of the four expected $d E / d x$ distributions represents the yield of particles. The free parameters in the fit are some small corrections at the nominal centre of the theoretical distributions for the pions, kaons and protons (labelled as $S_{\pi}, S_{K}, S_{p}$ ), a common correction factor $W$ to the width of the four particles distributions and four amplitudes $\left(A_{\pi}, A_{K}, A_{p}\right.$ and $\left.A_{e}\right)$ representing the relative contributions of each particle to the total. The role of the shift parameters $S_{\pi}$, $S_{K}, S_{p}$ is to correct for small calibration uncertainties. For the same reason, the correction factor $W$ is a final correction to the resolution.

In order to better handle low statistics particle extraction, the fitting method tries to 
minimise a $\chi^{2}$ function that has been built from a log-likelihood ratio as [12]:

$$
\chi^{2}=2 \cdot \sum_{i=1}^{n}\left[e_{i}-o_{i}+o_{i} \ln \left(\frac{o_{i}}{e_{i}}\right)\right]
$$

where $e_{i}$ and $o_{i}$ represent the expected and observed values at each bin $i$ of the $d E / d x$ distribution. In the case of $o_{i}=0$, the last term in equation (6.7) disappears. The minimisation of the $\chi^{2}$ is performed by a custom fitter, completely reimplemented in this thesis but inspired from [126]. For each of the correction parameters $\left(S_{\pi}, S_{K}, S_{p}\right)$ the fitting algorithm tries five different values at each step, iterating the procedure 35 times for each parameter to find the values that minimise the $\chi^{2}$ function. A grid search technique is at the same time applied to fit the parameters for the four particle amplitudes $A_{\pi}, A_{K}, A_{p}$ and $A_{e}$. $\mathrm{A}$ constant penalty factor has been added to equation (6.7) to constrain the free parameter regarding the correction to the position of the expected ionisation $\left(S_{i}\right.$, where $\left.\mathrm{i}=\pi, \mathrm{K}, \mathrm{p}\right)$ to be limited in the range $[-0.01,0.01]$. This range has been chosen to account for the expected uncertainty in the $\frac{d E}{d x}$ and not allow unphysical values: a justification of this range will be presented in section 6.7 . The correction at the position $S_{e}$ of the expected $\frac{d E}{d x}$ for the electron has not been considered in the fit due to the scarce yield of these particles.

Figure 6.8 shows a few examples of fitted $d E / d x$ distributions for positive (top) and negative (bottom) particles. Data are shown in black, the best fit obtained for the distribution of the four particles is superimposed. The plots on the left are shown for $x_{F}=0.05$ and $p_{T}=0.4 \mathrm{GeV} / c$. The plots on the right are for $x_{F}=0.2$ and $p_{T}=0.5 \mathrm{GeV} / c$. As the primary beam is made of protons, the production of protons is enhanced compared to the production of antiprotons. As $x_{F}$ increases, the production of protons increases, while the production of pions, kaons and antiprotons decreases. 

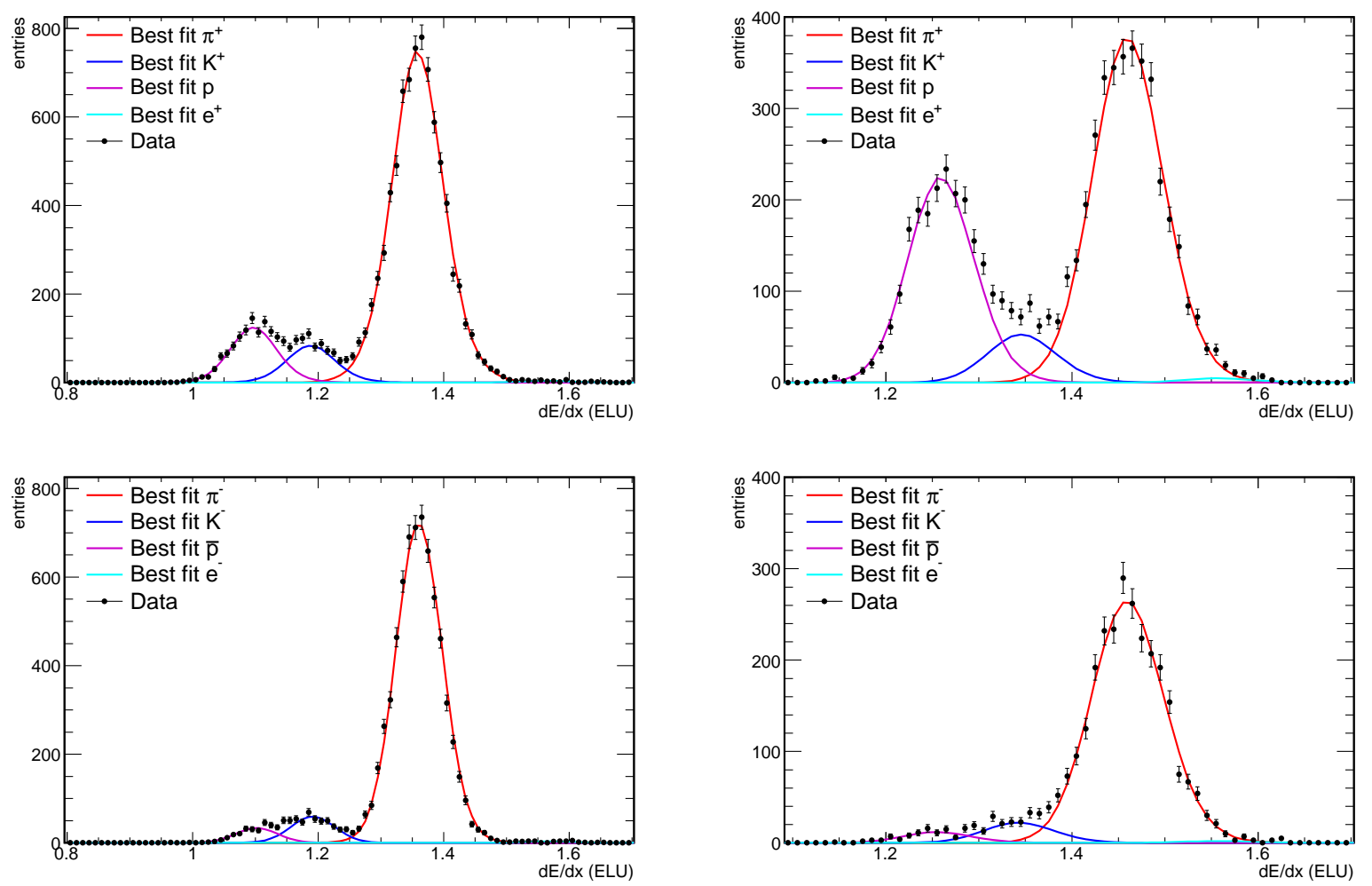

Figure 6.8: The figures show examples of fitted $d E / d x$ distributions for positive particles (top) and negative particles (bottom). The plots on the left are for $x_{F}=0.05$ and $p_{T}=0.4 \mathrm{GeV} / c$, while the plots on the right are for $x_{F}=0.2$ and $p_{T}=0.5 \mathrm{GeV} / c$. Data are shown in black; superimposed are the simulated best fit distributions for $\pi$ in red, kaons in blue, protons in purple and electrons in light blue. 

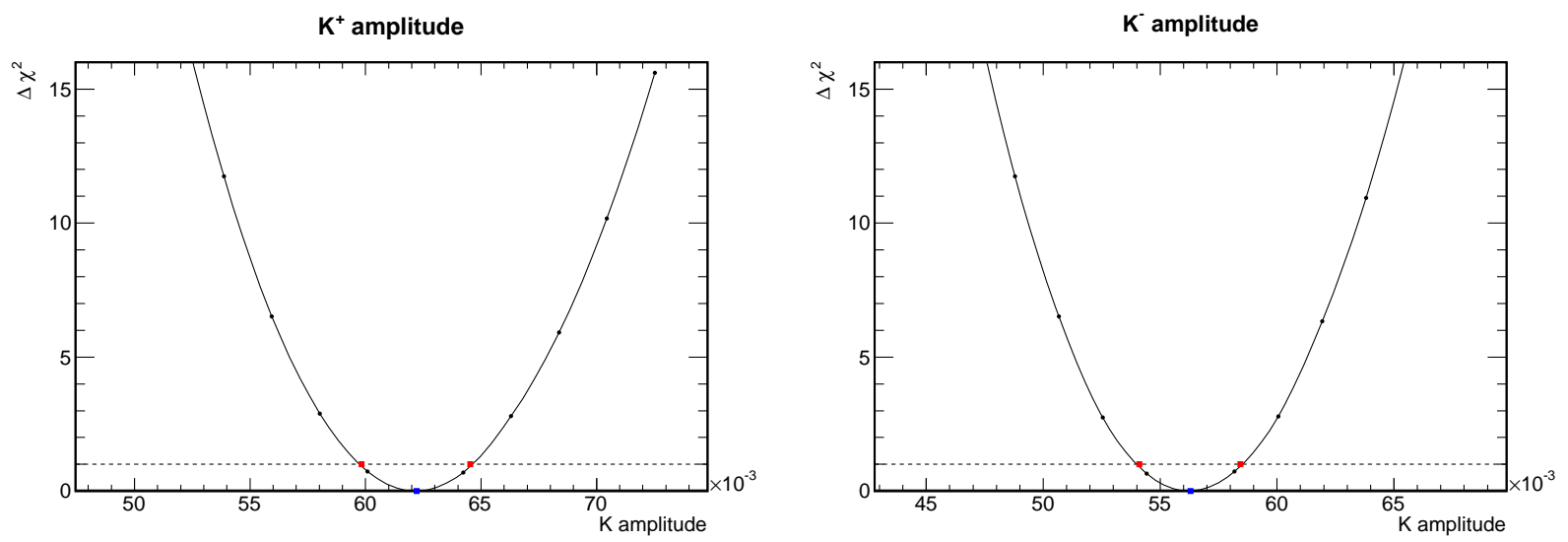

Figure 6.9: The projected value of the $\Delta \chi^{2}$ are shown as a function of kaon amplitude. The plot on the left is for $\mathrm{K}^{+}$, with $x_{F}=0.05$ and $p_{T}=0.2 \mathrm{GeV} / c$. On the right is for $\mathrm{K}^{-}$in the same bin. The best fit amplitude is indicated in blue. The up values are indicated in red and they correspond to the $\pm 1 \sigma$ error on the $A_{K}$ parameter. The black dots represent the values of the kaon amplitude for which the $\chi^{2}$ projection has been evaluated. The superimposed curve is a polynomial connection to the points.

\subsection{Errors on the fitted parameters}

The error on the parameters returned by the fit also needs to be calculated. In previous NA49 publications $[127,119]$, the errors on the pion yields have been treated as a Poisson error. In the case of kaons, suffering from very low statistic this is not the case. Correlation of the fitted parameters can modify the size of the error substantially. In this thesis, the errors on the fitted parameters have been evaluated by the projections of the $\chi^{2}$ curves, minimised in respect to all other variables, at different fixed values of the parameter being studied. The up-values for which the $\chi^{2}$ is equal to the minimum $\chi^{2}$ plus one unit represent the asymmetric error limits on the parameter (68\% C.L.). This technique is quite time consuming as a new minimisation of the $\chi^{2}$ versus all the other parameters has to be performed for each chosen value of the parameter being studied. For this reason, the minimisation has been performed scanning a limited amount of values. The up-values are evaluated assuming a linear interpolation between the two nearest measured $\chi^{2}$ points. In figure 6.9, the projection of the $\Delta \chi^{2}$ curve in the parameter amplitude of the kaons is shown for positive particles, bin $x_{F}=0.05, p_{T}=0.2$.

The number of kaons $N_{K}$ is derived by multiplying the amplitude of the kaons by the 
total number of events in the MC. In previous NA49 analysis, the error on $N_{K}$ was assumed to be $\sqrt{N_{K}}$. The errors on $N_{K}$ obtained in this thesis by the $\Delta \chi^{2}$ projection up-values are typically larger than $\sqrt{N_{K}}$ : figure 6.10 shows the ratio between the error on the number of kaons $N_{K}$ evaluated from the $\Delta \chi^{2}$ up-values and from the Poisson error $\left(\sqrt{N_{K}}\right)$ as a function of $x_{F}$. As the number of kaons is quite small in some $\left(x_{F}, p_{T}\right)$ bins, $\sqrt{N_{K}}$ cannot be used to evaluate the Poisson error.

In addition, the strong $x_{F}$ dependence can also be explained by looking at the ionisation loss curves in figure 6.2. At low momentum (small $x_{F}$ ), the ionisation loss curves of kaons and protons are close. As the momentum increases (and $x_{F}$ increases), the ionisation loss curves become more separate and the error on the kaons become closer to the statistical errors. At even bigger $x_{F}$, the ionisation curves of the particles tend to get closer again, making the shift parameter influence the particle yields. It is possible to quantify the separation between the peaks of the particles in the $d E / d x$ variable by defining a separation parameter $S$ which depends on the centre of the peak $C_{i}$ of the $i$ particle and the RMS of the distribution $\sigma_{i}$ :

$$
S=\left|\frac{C_{\mathrm{K}}-C_{\pi(p)}}{\sigma_{\mathrm{K}}+\sigma_{\pi(p)}}\right| .
$$

Figure 6.11 shows the separation parameter for kaons and pions (left) and kaons and protons (right) in the $\left(x_{F}, p_{T}\right)$ bins for positive particles. As explained above, at low $x_{F}$ kaons and protons are close (the expected distance between the peaks is $\approx 0.07$ ELU). Kaons and pions are distant $(\approx 0.2 \mathrm{ELU})$ but the resolution is quite poor at low momentum $(\sigma \approx 0.055$ ELU). At high $x_{F}$ the $d E / d x$ of the particles become closer (the distance between kaons and pions is $0.12 \mathrm{ELU}$ and the distance between kaons and protons is $0.08 \mathrm{ELU}$ ) but the resolution improves at higher momentum $(\sigma \approx 0.035 \mathrm{ELU})$. It is expected that the distance from the other particles will influence the kaon yield introducing correlation effects which vary bin-to-bin. The $\Delta \chi^{2}$ projection technique takes into account the correlations by minimising against all the other variables.

Figure 6.12 shows the measured percentage error on the number of kaons in the $\left(x_{F}\right.$, 

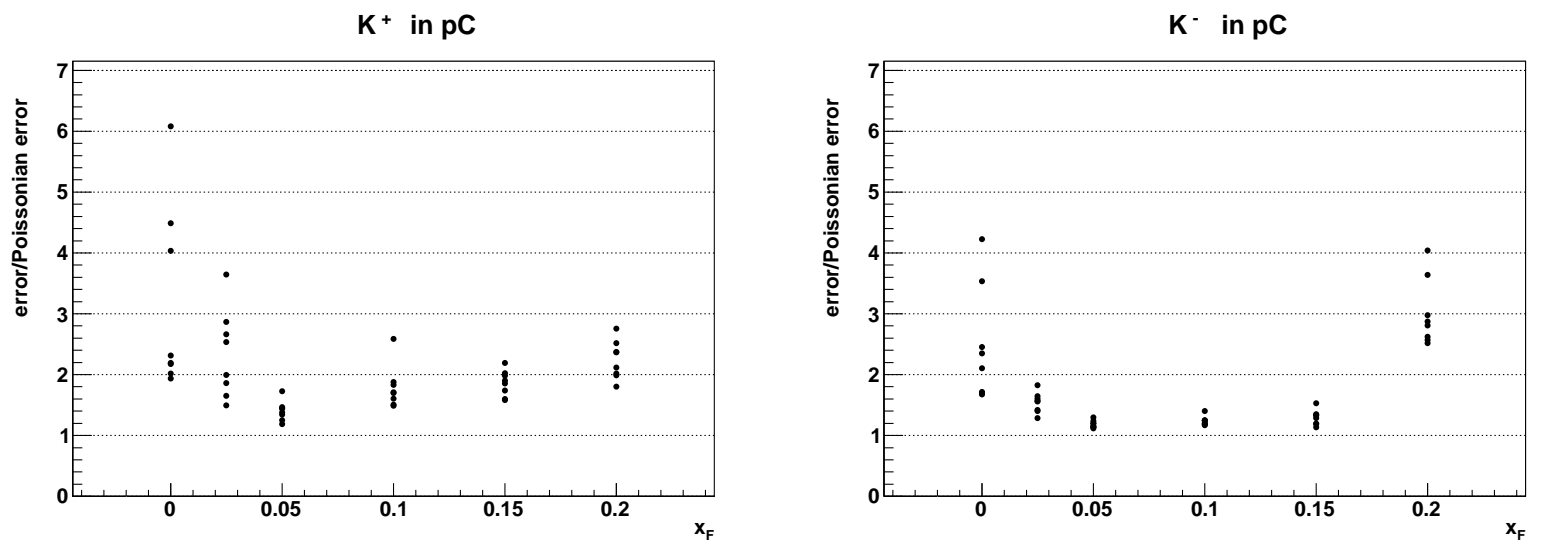

Figure 6.10: The kaon yield error divided by the Poisson error as a function of $x_{F}$ in the case of $\mathrm{K}^{+}$(left) and $\mathrm{K}^{-}$(right) in $\mathrm{pC}$.
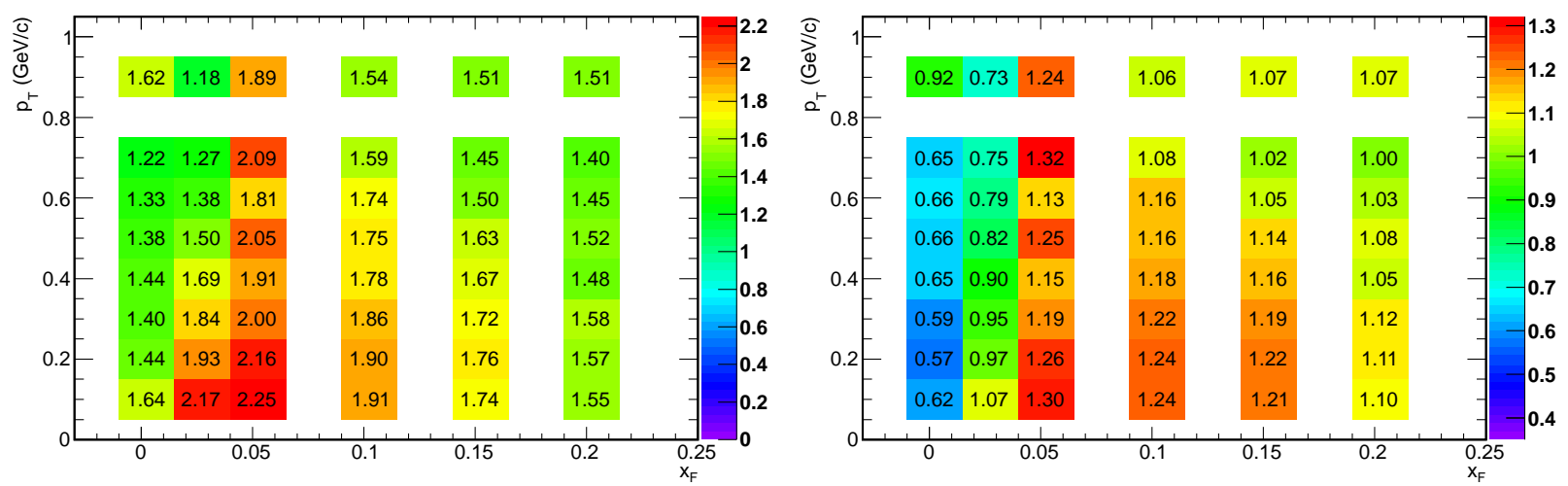

Figure 6.11: The separation parameter $S$ as defined in the text for kaons and pions on the left and for kaons and protons on the right. The plot is valid for positive particles and is a function of the $\left(x_{F}, p_{T}\right)$ bins.

$\left.p_{T}\right)$ bins.

In [128], a different approach to the minimisation and the error estimation is proposed: the minimisation of equation (6.7) is performed by the error covariant matrix. Agreement between the errors obtained from the covariant matrix and from the $\Delta \chi^{2}$ projections has been checked for a test sample. 

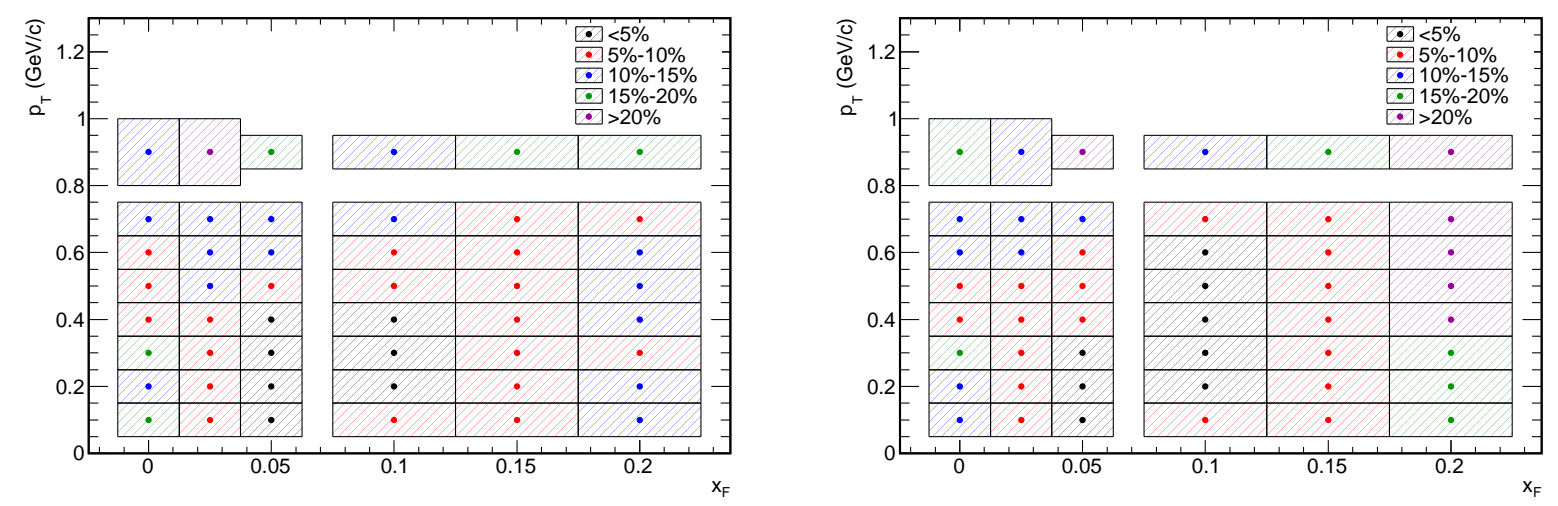

Figure 6.12: The measured percentage error on the number of kaons for positive particles (left) and for negative ones (right) as a function of $x_{F}$ and $p_{T}$ in $\mathrm{pC}$ interactions.

\subsection{Correlation of calibration parameters in $\mathrm{pC}$ and pp data}

Using the same $\left(x_{F}, p_{T}\right)$ binning scheme, particle identification in pp data has been achieved using the same technique. Results on the kaon production cross section in pp interactions are beyond the work in this thesis, although their interpretation could help to better motivate the $\mathrm{pC}$ results. The pp data offer a high statistics test sample as the number of collected events is 10 times larger than in the case of the pC run. NA49 official results on kaon production cross sections in pp have just been made available in [129] and are under journal referee review.

Figure 6.13 shows the percentage error on the number of kaons in the $\left(x_{F}, p_{T}\right)$ bins, as obtained by the $\Delta \chi^{2}$ projections. The size of the errors is much smaller than the one in the corresponding $\mathrm{pC}$ bins. The fitted parameters regarding the calibration uncertainties $\left(S_{i}\right.$, with $i=\pi, K, p$ and $W$ ) can be compared with the corresponding fitted parameters in $\mathrm{pC}$ interactions. Due to residual uncertainties on the time and gas gain calibration, the correction to the centre of the expected energy deposited distributions are not expected to be perfectly identical, but any deviation from the parameterised $d E / d x$ would be identical in both data samples. As the main limitation to the fitting of the kaons in the $\mathrm{pC}$ interactions is the lack of statistics, consistency of the calibration parameters $\left(S_{i}, W\right)$ between pC and 

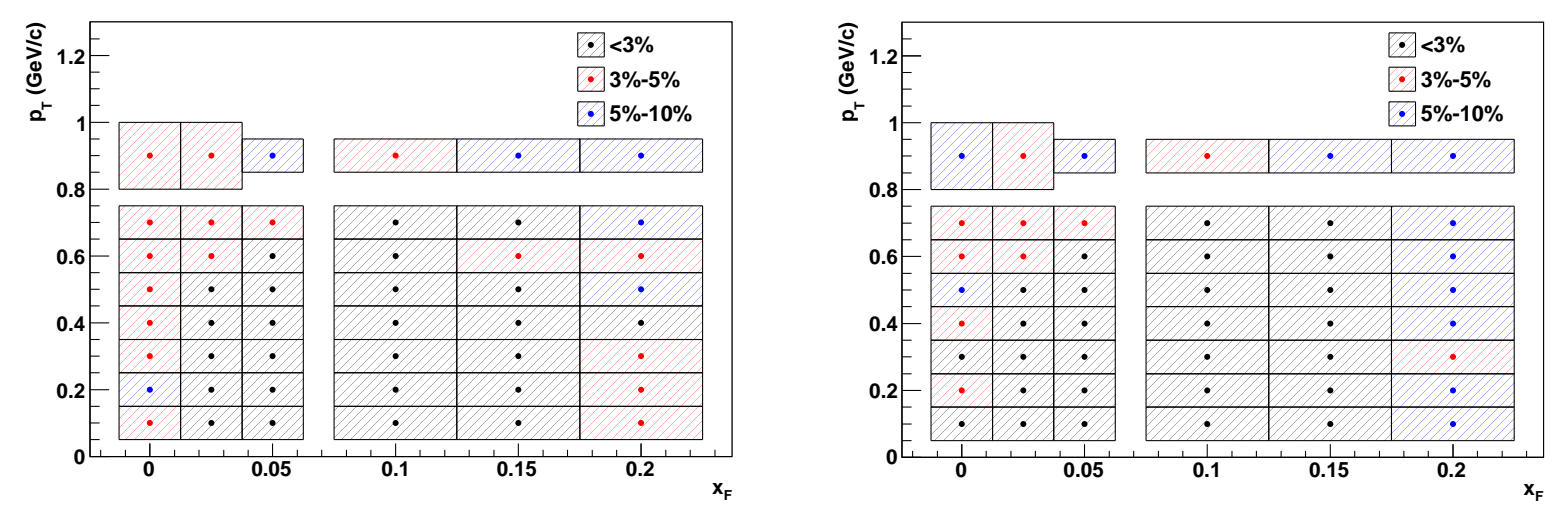

Figure 6.13: The measured percentage error on the number of kaons for positive particles on the left and for negative ones on the right as a function of $x_{F}$ and $p_{T}$ in pp interactions.

$\mathrm{pp}$ is desirable and can be checked at least for pions that are abundant also in the $\mathrm{pC}$ sample.

Figure 6.14 shows the correlation of the results obtained for the parameter $S_{\pi}$, representing the correction to the position of the expected ionisation loss for pions, in $\mathrm{pC}$ and pp. The errors on the corrections to the energy loss have been evaluated with the same technique explained in section 6.6. Figure 6.14 allows two considerations: overall the fitted parameters agree and the range of the corrections to the expected energy loss is within the $[-0.01,0.01]$ interval. This last consideration is important to justify the range allowed by the penalty term to the $\chi^{2}$ function in equation (6.7). As the parameterisation of the $d E / d x$ distribution is common to all particles and the range of velocities allowed by pions covers all the range of velocities for the other particles in the chosen binning for $x_{F}$ and $p_{T}$, this allows us to conclude that the same range of uncertainty in the correction to the expected ionisation has to be expected for protons and kaons, both particles for which a constrained fit is necessary. The correlation between the correction to the mean ionisation for kaons and protons in $\mathrm{pC}$ and pp do not show the same correlation as in the case of pions. This is due to lack in statistics in those particles (due to small production cross section). The poor results on the shift of protons and kaons in both pp and $\mathrm{pC}$ does not allow to use constraints from fits in pp to help reduce the uncertainties in $\mathrm{pC}$. A different approach to test the validity of the fits is given in section 6.8. 

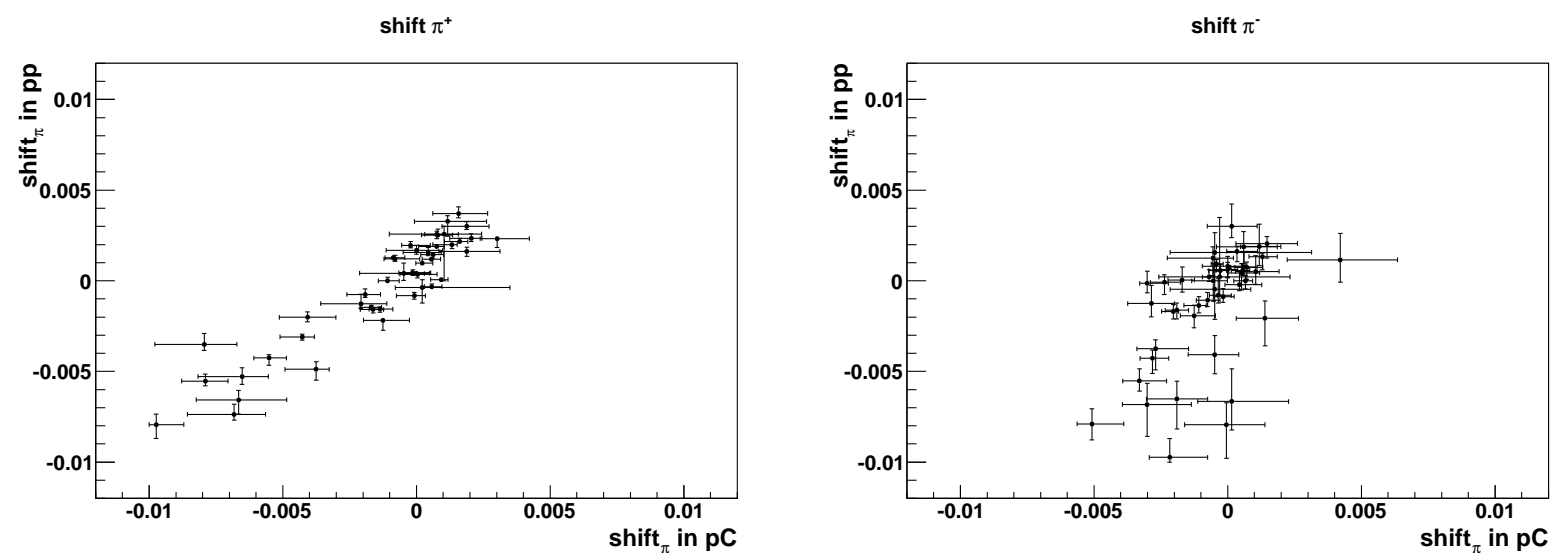

Figure 6.14: Correlation of the correction to expected $d E / d x$ for pions in $\mathrm{pC}$ and pp. On the left is for $\pi^{+}$and on the right is for $\pi^{-}$.

\subsection{Mock data fit in $\mathrm{pC}$ and $\mathrm{pp}$}

\subsubsection{Particle separation in case of low statistics}

A preliminary fit to a simulated $\frac{d E}{d x}$ spectrum built with known parameters but scaled to the data statistics has been performed in order to determine the sensitivity of the particle separation through a fit even in the presence of significant statistical fluctuations. The predicted spectrum for the energy loss for pions, kaons, proton and electrons was evaluated as described in section 6.4. To take into account the real statistical fluctuation of the data, each bin in the predicted spectrum has been successively Poisson fluctuated. This technique has been used to produce 500 independent distributions subject to the same magnitude of statistical fluctuations as the data. The $d E / d x$ spectra obtained, referred to as mock data, have subsequently been fitted according to the usual procedure. If the fitter does not have any bias, the known input parameters used to build the mock data $d E / d x$ distributions should be returned by the fit.

An example of the distributions obtained for the fitted parameters are presented in figure 6.15 both for $\mathrm{pC}$ and $\mathrm{pp}$ interactions. In the case shown, the chosen bin is for positive particles, $x_{F}=0, p_{T}=0.2 \mathrm{GeV}$ and $\phi=90^{\circ}$. The input parameters have been chosen to be the same for $\mathrm{pC}$ and $\mathrm{pp}$ in order to better compare the impact of the statistics in 
the fit. In general the parameters have been well determined by the fit. The RMS of the distributions in the case of $\mathrm{pC}$ is bigger than for $\mathrm{pp}$ due to the different statistics, but scales by $\sqrt{10}$, where 10 represents approximatively the factor in statistics by which the pp set is higher than the $\mathrm{pC}$ set. In the $\mathrm{pC}$ case, the correction to the position of the kaons is not well determined by the fit and often is put at the border of the allowed range. Nevertheless, even if the position of kaons and protons is not well determined, no substantial bias in the measurement of the amplitude of the kaons (and thus in their yield) is noticed. The mock data fit performed guarantees the feasibility of the particle separation through the fitting technique adopted.

\subsubsection{Cross check of the kaon yield errors}

For the pC interactions, once having performed the fit to the data, 500 Poisson fluctuated mock data fits were performed where the input used is the best fit to the real data. This was done for each $\left(x_{F}, p_{T}\right)$ bin. It is possible to evaluate the error on the parameters from the RMS of the distributions of the fitted parameters (as shown in figure 6.15). This method allows a cross check on the size of the errors by comparison to the error obtained from the $\chi^{2}$ projection. Figure 6.16 shows the percentage expected errors on kaon yield as obtained

with the mock data fit method for the different $\left(x_{F}, p_{T}\right)$ bins. Although some differences from the percentage errors obtained from the $\chi^{2}$ projections (figure 6.12 ) are present, the overall size of the error agrees.

\subsection{Double differential cross section}

The invariant double differential cross section given as a function of $x_{F}$ and $p_{T}$ is defined by the formula:

$$
f\left(x_{F}, p_{T}\right)=E\left(x_{F}, p_{T}\right) \cdot \frac{d^{3} \sigma}{d p^{3}}\left(x_{F}, p_{T}\right)
$$



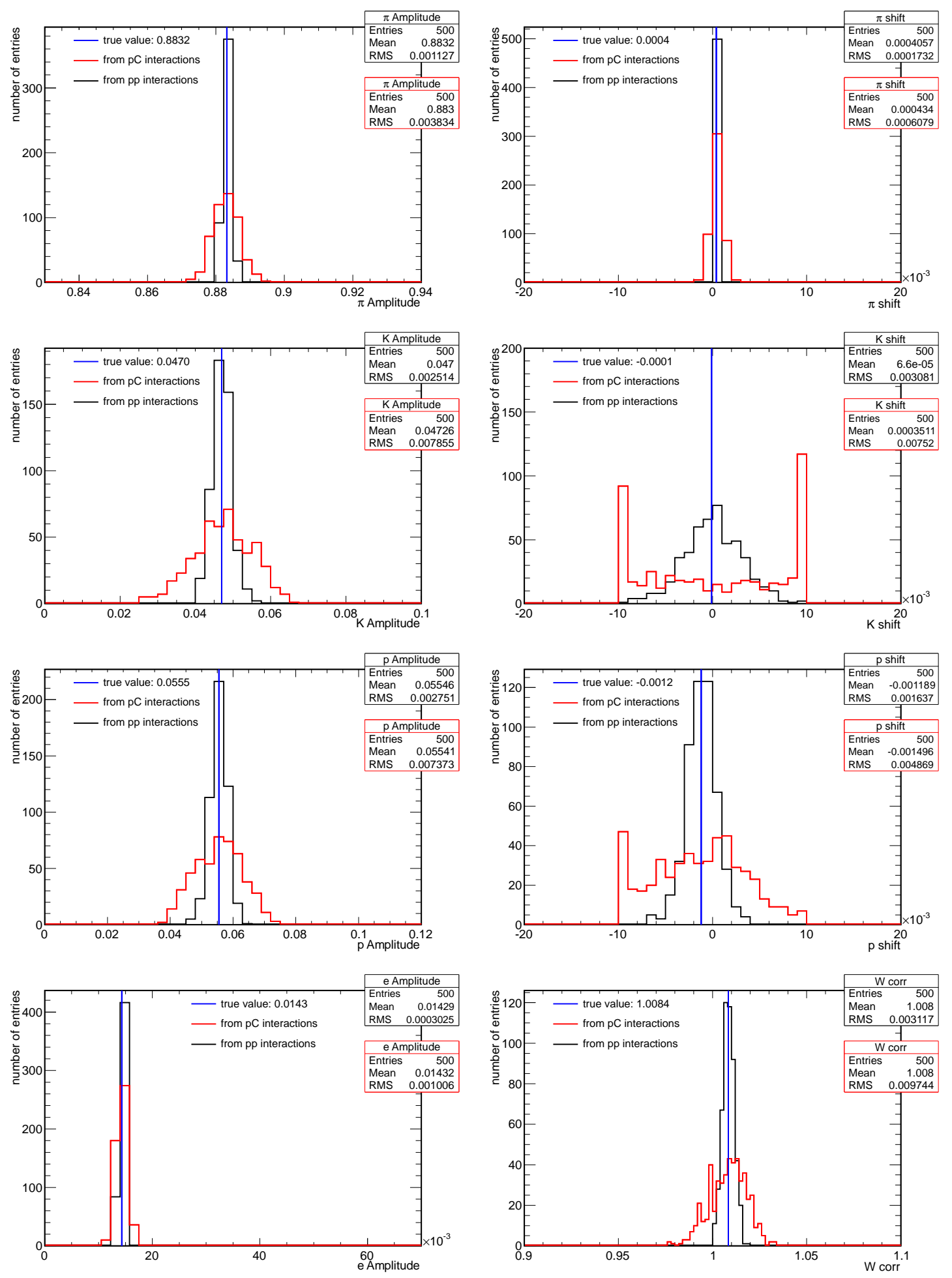

Figure 6.15: The figures show the distributions for the parameter results obtained by the fits of the 500 Poisson fluctuated $d E / d x$ spectra for both $\mathrm{pC}$ (in red) and pp (in black) interactions in the bin at $x_{F}=0$ and $p_{T}=0.2 \mathrm{GeV}$. From the top the distributions show the pion amplitude, the pion position, the kaon amplitude, the kaon position, the proton amplitude, the proton position, the electron amplitude and the width correction factor. The blue line in the plots represents the true value used in the input $d E / d x$ spectrum. 

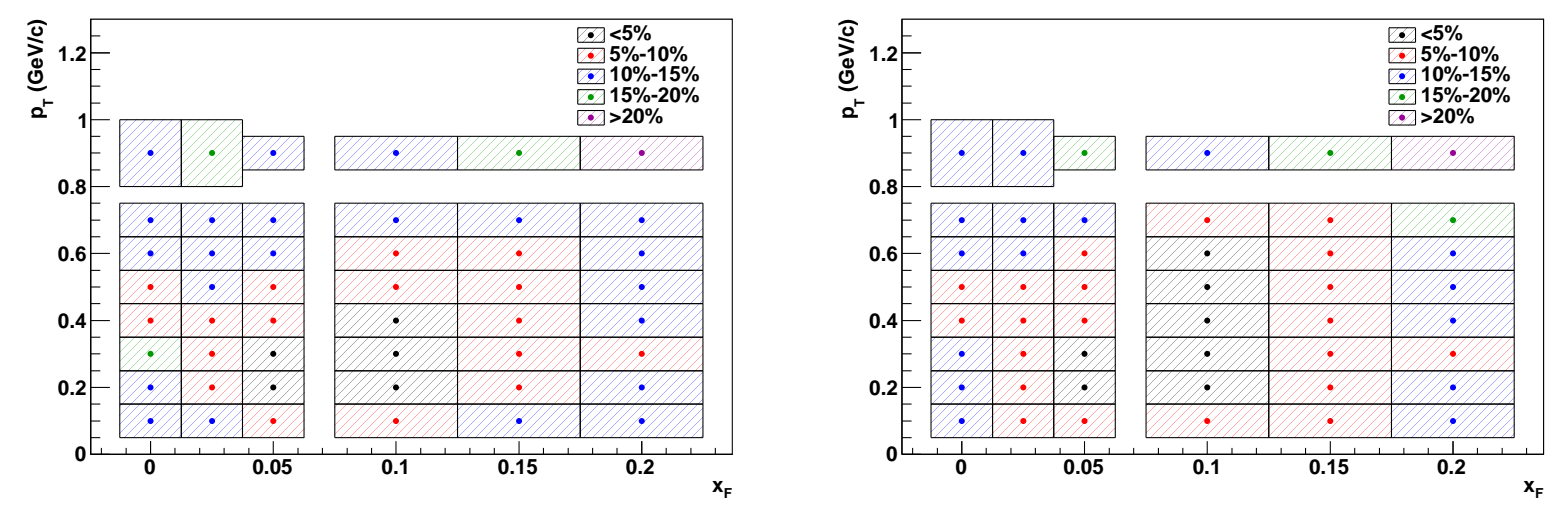

Figure 6.16: The plots show the expected percentage error on the number of kaons for positive particles on the left and for negative ones on the right as a function of $x_{F}$ and $p_{T}$ in $\mathrm{pC}$ interactions. The errors have been determined as the RMS of the distributions of the fitted parameters when building mock data using the parameters obtained from the fit of real data in the same $x_{F}$ and $p_{T}$ bin as input.

where $d p^{3}$ is the infinitesimal volume element in three dimensional momentum space. The cross section measurement has to be performed in the finite volume element $\Delta p^{3}=\Delta p^{3}\left(x_{F}, p_{T}\right)$ and the measured double differential cross section $f_{\text {meas }}$ can be written as:

$$
f_{\text {meas }}\left(\Delta p^{3}\right)=E\left(\Delta p^{3}\right) \cdot \frac{\sigma_{\text {trigger }}}{N_{\mathrm{ev}}} \cdot \frac{\Delta n\left(\Delta p^{3}\right)}{\Delta p^{3}}
$$

where $\Delta n\left(\Delta p^{3}\right)$ represents the observed number of identified kaons in the bin $\Delta p^{3}, N_{\mathrm{ev}}$ represents the total number of observed events and $\sigma_{\text {trigger }}$ is a factor that takes into account that events are collected only when they do produce some interactions and is proportional to the quantity $N_{\text {ev }} / N_{\text {beam. }}$. The value of $\sigma_{\text {trigger }}$ has been evaluated [117] to be $\approx 210 \mathrm{mb}$. Uncertainties in the trigger cross section will cause an overall normalisation issue: the effect is considered in section 6.11. In the calculation of $f_{\text {meas }}$ a correction factor to restore the full $2 \pi$ range in $\phi$ needs to be performed. In the evaluation of the error on the inclusive cross section, it has been assumed that the only statistical uncertainty comes from the number of kaons $\Delta n\left(p^{3}\right)$ in equation (6.10). 


\subsection{Corrections}

In order to compute the differential invariant cross section, a set of correction factors (computed by the NA49 collaborators using a private Monte Carlo or data) need to be applied:

- Empty target corrections: despite the preliminary cuts on the Beam Position Detectors and on the $z$ position of the fitted track vertex, a small residual contribution to the particle yield from the non-target material is present. For example, low multiplicity events, that have a bad vertex resolution are not cut by the $z$ cuts. A correction can be obtained directly from the available empty target data sample, as described in [122]. This correction is at maximum $6 \%$, decreasing slowly with track momentum.

- Re-interaction in the target: as the target is only $1.5 \%$ interaction lengths thick, the secondary interaction probability is very small. The reinteraction effects will be to move some of the particles towards lower $x_{F}$ and the total multiplicity of particles will slightly increase. The correction, smaller than $2 \%$ even at low momentum, has been evaluated by NA49 collaborators [130] using the PYTHIA event generator [131].

- Kaon weak decay: the decay length of the kaons is slightly more than $40 \mathrm{~m}$ at the lowest momentum studied $\left(c \tau_{\mathrm{K}} \approx 3.7 \mathrm{~m}[12]\right.$ and $\left.\gamma \approx 10\right)$. The decay products are not reconstructed to the primary vertex in the reconstruction program. All decaying kaons that have travelled less than a minimum number of pad rows in the first TPC located on their trajectory have to be considered lost. This correction can be up to $9 \%$ for low momentum tracks.

- Absorption in the detector material: the correction for kaons interacting in the detector material downstream of the target is determined using the GEANT simulation of the NA49 detector. This correction can be maximum $2 \%$ at higher track momentum.

- Feed-down: in theory, any kaon yield contribution coming from particles which decay weakly into kaons has to be subtracted. In practise, only the $\Omega^{-}$and $D$ mesons could contribute to the kaon yields and their production can be neglected. 
- Trigger bias: the trigger has efficiency of only $93 \%$ for selection of inelastic events. This is due to the fact that secondary produced particles can hit the anti-counter S4 and cause the event to be vetoed. This probability increases for higher momentum particles. The correction is described in [126] and is obtained by creating a software artificial ring around the S4 counter with the same area. The density of tracks firing the ring is checked in data and MC as a function of the particle momentum. A weight is obtained from the MC ratio of the density of tracks firing the S4 counter divided by the density of tracks firing the artificial ring in the $\mathrm{MC}$ as a function of $p_{z}$. If a track in an event hits the ring, the weight is evaluated from the track $p_{z}$. The same weight (which can be $+2 \%$ ) is applied to all the tracks of the event as this event has to compensate for events vetoed by the S4 counter.

- Binning: in general the cross section value obtained in the $\Delta p^{3}$ bin is valid at the mean of the bin, but not at its centre. To correct for the effect of finite bin size, knowledge of the cross section shape is necessary: the correction is determined using the second derivatives of the particle density distribution as a function of $x_{F}$ and $p_{T}$.

The correction factors are applied as a multiplicative number on the cross section measurement. Figure 6.17 shows three correction factors as a function of $x_{F}$ for $\mathrm{K}^{+}$(left) and $\mathrm{K}^{-}$(right). The correction factor applied to remove the effect of the empty target is shown in red, the trigger bias is shown in blue and the reinteraction in the target is shown in green.

\subsection{Systematic errors}

Various potential sources of systematic errors have been considered: the systematic errors have been found to be quite small $(4.2 \%)$ compared to the statistical errors, which are shown in figure 6.12. The evaluation of some of the systematic uncertainties is done using data available in the stages before the $\mu \mathrm{DST}$ s and have been determined by NA49 collab- 

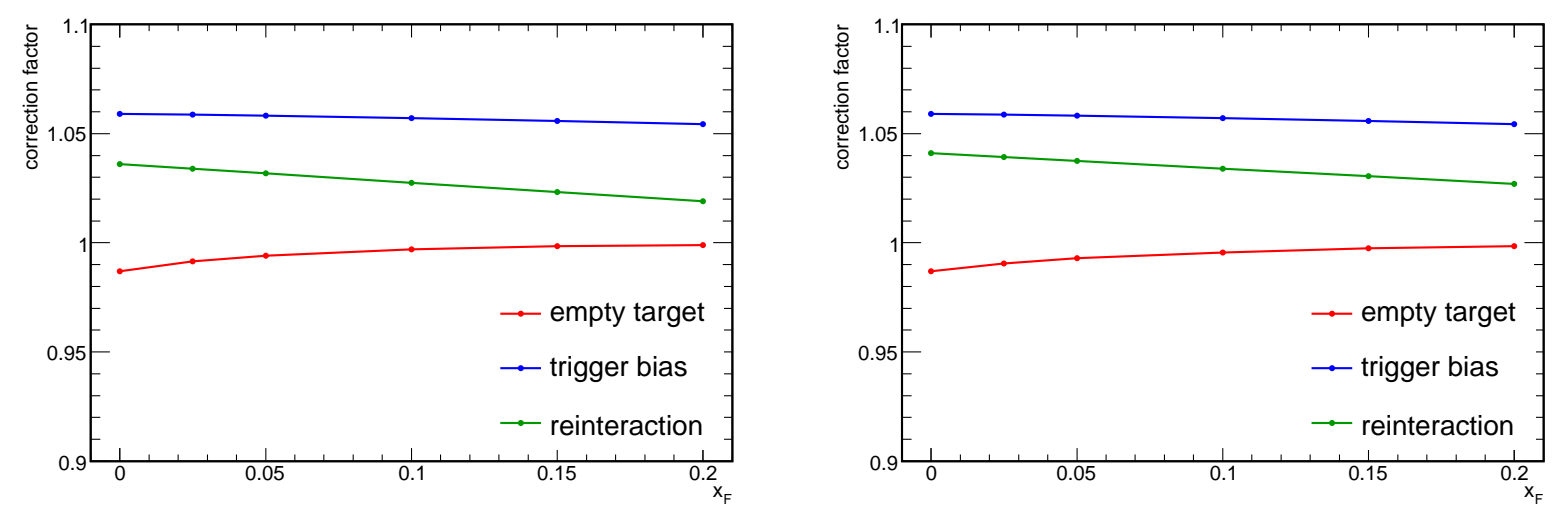

Figure 6.17: The figure shows some corrections for the invariant cross section for $\mathrm{K}^{+}$(left) and $\mathrm{K}^{-}$(right). The empty target correction is in red (it is the same for $\mathrm{K}^{+}$and $\mathrm{K}^{-}$), trigger bias is in blue (it is the same for $\mathrm{K}^{+}$and $\mathrm{K}^{-}$) and the target reinteraction correction is in green (it differs between $\mathrm{K}^{+}$and $\mathrm{K}^{-}$).

orators. Table 6.1 shows the size of the errors for the $\left(x_{F}, p_{T}\right)$ bins. Tracking efficiency uncertainty [119] and the errors on the correction factors presented in section 6.10 are quite small [129].

The overall normalisation uncertainty is $2.6 \%$; the size of this error is mainly determined by the uncertanty on the trigger cross section, which is $2.5 \%$, as evaluated in [119]. Another contribution to the normalisation comes from the uncertainty on the momentum. The momentum of the tracks in the MC has been smeared by the resolution $d p / p^{2}=7.0 \times 10^{-4}(\mathrm{GeV} / c)^{-1}$. The average percentage variation in the number of tracks selected in the $\left(x_{F}, p_{T}, \phi\right)$ bins has been evaluated to be $0.5 \%$. The uncertainty of $0.5 \%$ needs to be added in quadrature to the trigger cross section uncertainty for the overall normalization.

The uncertainty on the $d E / d x$ modelling has been evaluated to be $3.1 \%$. As explained in section 6.4, equation (6.5) is valid only if the number of charge clusters sampled along the track $N_{p}$ does not depend on the ionisation loss. Figure 6.7 shows the resolution of the specific ionisation as a function of the $d E / d x$ and of the number of points sampled. The resolution is better at higher $d E / d x$ and higher $N_{p}$, suggesting a correlation between higher $d E / d x$ (mainly pions) and the higher number of points sampled. As all pC data tracks are 


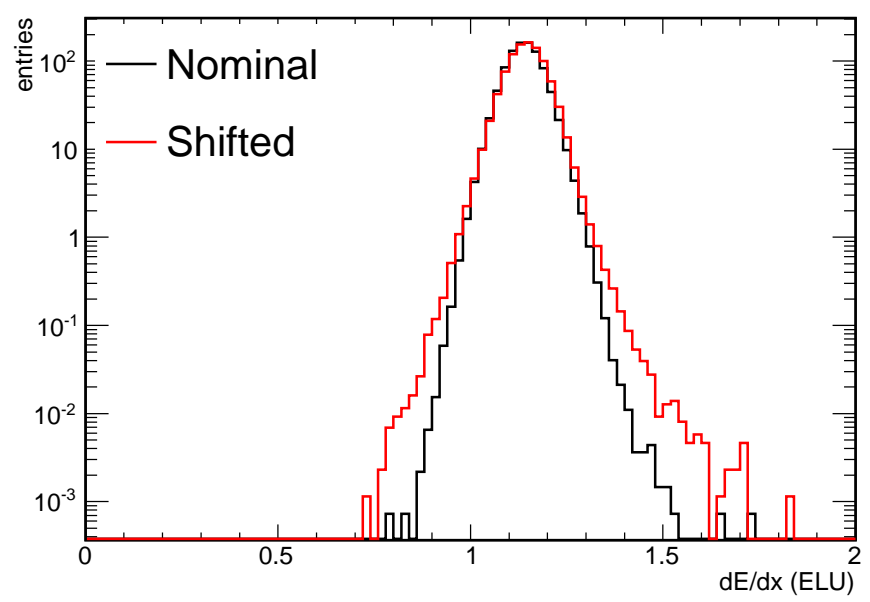

Figure 6.18: The modelling of the $d E / d x$ for kaons in a particular $\left(x_{F}, p_{T}\right)$ bin is shown in black. The systematically modified spectrum is shown in red.

used to construct the inputs for the $\mathrm{MC}$ of the different particles, tracks that are truly pions will have a better measured resolution $\sigma_{\text {data }}$ compared to kaons. Nevertheless in the $\mathrm{MC}$, the resolution of pions (that are the most conspicuous particles) would be used as an input for the resolution of the kaons, that in reality should have a slightly worse resolution. It is possible to evaluate the size of this effect by using the high statistics pp data as an input for the MC. As the statistics of the pp sample is high, it is possible to use only those tracks that have a $d E / d x$ within $\pm 1 \sigma$ from the expected value of each particle type as an input to the $\mathrm{MC}$ for that particular particle. In this way, the simulated pions will be using a sample of pure pions as an input for the $d E / d x$ distribution; in the same fashion kaons are simulated using mainly kaons. The fit to the $\mathrm{pC}$ data with the newly simulated $\mathrm{MC}$ was performed: the number of pions was found to be consistent within $0.5 \%$. Instead, the number of kaons was found to be systematically higher in the new MC by 3.1\%. Figure 6.18 shows the effect of the $d E / d x$ modelling for simulated kaons in a typical $\left(x_{F}, p_{T}\right)$ bin.

\subsection{Results}

Figures 6.19 and 6.20 show the results of the evaluation of the inclusive invariant cross section for positive and negative kaons as a function of $x_{F}$ and $p_{T}$. The error for each cross 


\begin{tabular}{|c|c|}
\hline Systematic & error $(\%)$ \\
\hline Normalisation & 2.6 \\
\hline$d E / d x$ modelling & 3.1 \\
\hline Tracking efficiency & 0.5 \\
\hline Trigger bias & 1.0 \\
\hline Detector absorption ) & \\
\hline Kaon decay & 1.0 \\
\hline Target reinteraction & \\
\hline Binning & 0.5 \\
\hline Summed in quadrature & 4.2 \\
\hline
\end{tabular}

Table 6.1: The table shows the sources of systematic errors relevant to the determination of the kaon inclusive invariant production cross section.

section value is the sum in quadrature of the statistical error and the $4.2 \%$ systematic error. To guide the eyes cross section results as a function of $x_{F}$ have been fitted with a function of the form:

$$
\left(a+b \cdot x_{F}\right) \cdot e^{-c \cdot x_{F}}
$$

Each curve at fixed $p_{T}$ has been fitted independently from the others, a global fit to the cross section has not been attempted. In a similar fashion, each cross section value as a function of $p_{T}$ for different values of $x_{F}$ has been fitted to a curve of the form:

$$
\left(a+b \cdot p_{T}\right) \cdot e^{-c \cdot p_{T}^{\frac{3}{2}}}
$$

In general $a$ and $b$ are expected to be a function of $x_{F}$. Tables 6.2 and 6.3 report the cross section values and errors for the bins in $\left(x_{F}, p_{T}\right)$.

\subsection{1 $\mathrm{K}^{+} / \mathrm{K}^{-}$ratios}

Figure 6.21 shows the ratio of the $\mathrm{K}^{+}$to the $\mathrm{K}^{-}$cross sections as a function of $x_{F}$ and $p_{T}$. Table 6.4 shows the values for the $\mathrm{K}^{+} / \mathrm{K}^{-}$ratios. The $p_{T}$ integrated distributions will be presented in the next chapter. 


\begin{tabular}{|c|r|r|r|r|r|c|}
\hline \multicolumn{7}{|c|}{$f\left(x_{F}, p_{T}\right)$} \\
\hline$p_{T} \backslash x_{F}$ & \multicolumn{1}{|c|}{0.00} & 0.025 & 0.05 & \multicolumn{1}{c|}{0.1} & \multicolumn{1}{c|}{0.15} & 0.2 \\
\hline 0.1 & $32.70_{-5.20}^{+5.35}$ & $25.78_{-1.95}^{+2.15}$ & $28.98_{-1.64}^{+1.67}$ & $18.91_{-1.20}^{+1.31}$ & $12.97_{-1.13}^{+1.16}$ & $9.93_{-1.53}^{+1.14}$ \\
0.2 & $30.35_{-3.19}^{+5.09}$ & $23.13_{-1.69}^{+1.85}$ & $19.52_{-1.10}^{+1.11}$ & $15.27_{-0.85}^{+0.95}$ & $10.72_{-0.86}^{+0.83}$ & $8.92_{-1.06}^{+1.08}$ \\
0.3 & $16.79_{-2.53}^{+4.32}$ & $17.63_{-1.29}^{+1.14}$ & $15.87_{-0.94}^{+0.92}$ & $12.53_{-0.79}^{+0.71}$ & $9.05_{-0.59}^{+0.79}$ & $8.56_{-0.63}^{+0.98}$ \\
0.4 & $17.24_{-1.09}^{+1.55}$ & $11.93_{-1.12}^{+0.87}$ & $10.84_{-0.71}^{+0.66}$ & $8.83_{-0.52}^{+0.53}$ & $6.58_{-0.51}^{+0.52}$ & $4.66_{-0.58}^{+0.67}$ \\
0.5 & $9.75_{-0.85}^{+0.98}$ & $7.40_{-1.24}^{+0.69}$ & $7.50_{-0.66}^{+0.60}$ & $6.06_{-0.55}^{+0.50}$ & $4.75_{-0.32}^{+0.37}$ & $4.01_{-0.47}^{+0.45}$ \\
0.6 & $6.66_{-0.57}^{+0.69}$ & $5.76_{-0.75}^{+0.68}$ & $4.56_{-0.57}^{+0.47}$ & $4.28_{-0.48}^{+0.31}$ & $3.82_{-0.36}^{+0.30}$ & $3.16_{-0.41}^{+0.38}$ \\
0.7 & $4.71_{-0.53}^{+0.54}$ & $4.10_{-0.46}^{+0.74}$ & $3.12_{-0.38}^{+0.39}$ & $2.70_{-0.46}^{+0.32}$ & $2.74_{-0.30}^{+0.26}$ & $2.85_{-0.31}^{+0.27}$ \\
0.9 & $2.06_{-0.24}^{+0.31}$ & $1.51_{-0.25}^{+0.40}$ & $1.65_{-0.31}^{+0.23}$ & $1.27_{-0.15}^{+0.17}$ & $1.03_{-0.17}^{+0.20}$ & $0.94_{-0.19}^{+0.16}$ \\
\hline
\end{tabular}

Table 6.2: Inclusive invariant cross section $f\left(x_{F}, p_{T}\right)$ in $\mathrm{mb} /\left(\mathrm{GeV}^{2} / c^{3}\right)$ for $\mathrm{K}^{+}$in $\mathrm{pC}$ collisions at $158 \mathrm{GeV} / c$ beam momentum. The asymmetric errors are also given.

\begin{tabular}{|c|c|r|r|r|c|c|}
\hline \multicolumn{7}{|c|}{$f\left(x_{F}, p_{T}\right)$} \\
\hline$p_{T} \backslash x_{F}$ & \multicolumn{1}{|c|}{0.00} & \multicolumn{1}{c|}{0.025} & 0.05 & \multicolumn{1}{c|}{0.1} & 0.15 & 0.2 \\
\hline 0.1 & $21.02_{-2.18}^{+3.12}$ & $19.07_{-1.63}^{+1.60}$ & $15.86_{-1.05}^{+1.02}$ & $11.88_{-0.81}^{+0.83}$ & $6.58_{-0.64}^{+0.57}$ & $4.34_{-0.63}^{+0.55}$ \\
0.2 & $16.60_{-2.34}^{+2.48}$ & $15.97_{-1.08}^{+1.08}$ & $14.10_{-0.80}^{+0.81}$ & $9.48_{-0.55}^{+0.56}$ & $6.99_{-0.48}^{+0.48}$ & $4.26_{-0.46}^{+0.45}$ \\
0.3 & $11.88_{-1.74}^{+2.31}$ & $12.21_{-0.86}^{+0.86}$ & $10.47_{-0.67}^{+0.62}$ & $7.15_{-0.41}^{+0.41}$ & $5.09_{-0.39}^{+0.37}$ & $3.31_{-0.34}^{+0.35}$ \\
0.4 & $10.60_{-0.78}^{+0.81}$ & $9.61_{-0.71}^{+0.80}$ & $6.68_{-0.49}^{+0.44}$ & $5.62_{-0.32}^{+0.33}$ & $3.71_{-0.28}^{+0.27}$ & $2.15_{-0.25}^{+0.30}$ \\
0.5 & $7.84_{-0.71}^{+0.78}$ & $6.68_{-0.49}^{+0.58}$ & $5.03_{-0.40}^{+0.42}$ & $3.95_{-0.25}^{+0.23}$ & $2.79_{-0.20}^{+0.20}$ & $1.79_{-0.22}^{+0.19}$ \\
0.6 & $3.82_{-0.56}^{+0.48}$ & $3.52_{-0.42}^{+0.40}$ & $3.23_{-0.29}^{+0.28}$ & $2.82_{-0.17}^{+0.18}$ & $1.75_{-0.16}^{+0.18}$ & $1.08_{-0.20}^{+0.12}$ \\
0.7 & $2.39_{-0.29}^{+0.29}$ & $2.61_{-0.28}^{+0.32}$ & $2.27_{-0.33}^{+0.27}$ & $1.58_{-0.13}^{+0.12}$ & $1.24_{-0.11}^{+0.12}$ & $0.80_{-0.10}^{+0.10}$ \\
0.9 & $1.01_{-0.16}^{+0.18}$ & $1.17_{-0.13}^{+0.13}$ & $0.68_{-0.16}^{+0.12}$ & $0.57_{-0.09}^{+0.08}$ & $0.45_{-0.08}^{+0.08}$ & $0.30_{-0.06}^{+0.09}$ \\
\hline
\end{tabular}

Table 6.3: Inclusive invariant cross section $f\left(x_{F}, p_{T}\right)$ in $\mathrm{mb} /\left(\mathrm{GeV}^{2} / c^{3}\right)$ for $\mathrm{K}^{-}$in $\mathrm{pC}$ collisions at $158 \mathrm{GeV} / c$ beam momentum. The asymmetric errors are also given.

\begin{tabular}{|c|c|c|c|c|c|c|}
\hline \multicolumn{7}{|c|}{$f\left(x_{F}, p_{T}\right)$} \\
\hline$p_{T} \backslash x_{F}$ & 0.00 & 0.025 & 0.05 & 0.1 & 0.15 & 0.2 \\
\hline 0.1 & $1.56_{-0.30}^{+0.34}$ & $1.35_{-0.15}^{+0.16}$ & $1.83_{-0.16}^{+0.16}$ & $1.59_{-0.15}^{+0.16}$ & $1.97_{-0.26}^{+0.25}$ & $2.29_{-0.48}^{+0.39}$ \\
0.2 & $1.83_{-0.32}^{+0.41}$ & $1.45_{-0.14}^{+0.15}$ & $1.38_{-0.11}^{+0.11}$ & $1.61_{-0.13}^{+0.14}$ & $1.53_{-0.16}^{+0.16}$ & $2.09_{-0.33}^{+0.34}$ \\
0.3 & $1.41_{-0.30}^{+0.46}$ & $1.44_{-0.15}^{+0.14}$ & $1.52_{-0.13}^{+0.13}$ & $1.75_{-0.15}^{+0.14}$ & $1.78_{-0.18}^{+0.20}$ & $2.58_{-0.33}^{+0.40}$ \\
0.4 & $1.63_{-0.16}^{+0.19}$ & $1.24_{-0.15}^{+0.14}$ & $1.62_{-0.16}^{+0.15}$ & $1.57_{-0.13}^{+0.13}$ & $1.77_{-0.19}^{+0.19}$ & $2.16_{-0.37}^{+0.43}$ \\
0.5 & $1.24_{-0.16}^{+0.18}$ & $1.11_{-0.20}^{+0.14}$ & $1.49_{-0.18}^{+0.17}$ & $1.53_{-0.17}^{+0.15}$ & $1.71_{-0.17}^{+0.18}$ & $2.24_{-0.38}^{+0.35}$ \\
0.6 & $1.75_{-0.30}^{+0.28}$ & $1.64_{-0.29}^{+0.27}$ & $1.41_{-0.22}^{+0.19}$ & $1.52_{-0.19}^{+0.15}$ & $2.18_{-0.28}^{+0.28}$ & $2.92_{-0.66}^{+0.48}$ \\
0.7 & $1.97_{-0.33}^{+0.33}$ & $1.57_{-0.25}^{+0.34}$ & $1.37_{-0.26}^{+0.24}$ & $1.71_{-0.32}^{+0.24}$ & $2.21_{-0.31}^{+0.30}$ & $3.56_{-0.60}^{+0.55}$ \\
0.9 & $2.03_{-0.40}^{+0.47}$ & $1.29_{-0.25}^{+0.37}$ & $2.45_{-0.74}^{+0.55}$ & $2.21_{-0.42}^{+0.44}$ & $2.32_{-0.57}^{+0.61}$ & $3.09_{-0.87}^{+1.07}$ \\
\hline
\end{tabular}

Table 6.4: The table reports the ratio of $K^{+}$to the $K^{-}$production cross section as a function of $x_{F}$ and $p_{T}$. 


\subsection{Conclusion}

The kaon inclusive invariant cross sections have been measured for an extensive $\left(x_{F}, p_{T}\right)$ phase space. Particular effort was put in the fitting method to the $d E / d x$ to obtain particle identification. Preliminary sensitivity studies were performed using the MC in order to study the particle identification performance given the low statistics of the data used. Two alternative methods to measure the statistical errors on the number of kaons have been proposed, obtaining consistent results. The measurements performed in this analysis will be used in the next chapter and will be compared to existing measurements by other experiments and to the MC expectation of the MINOS beamline. 

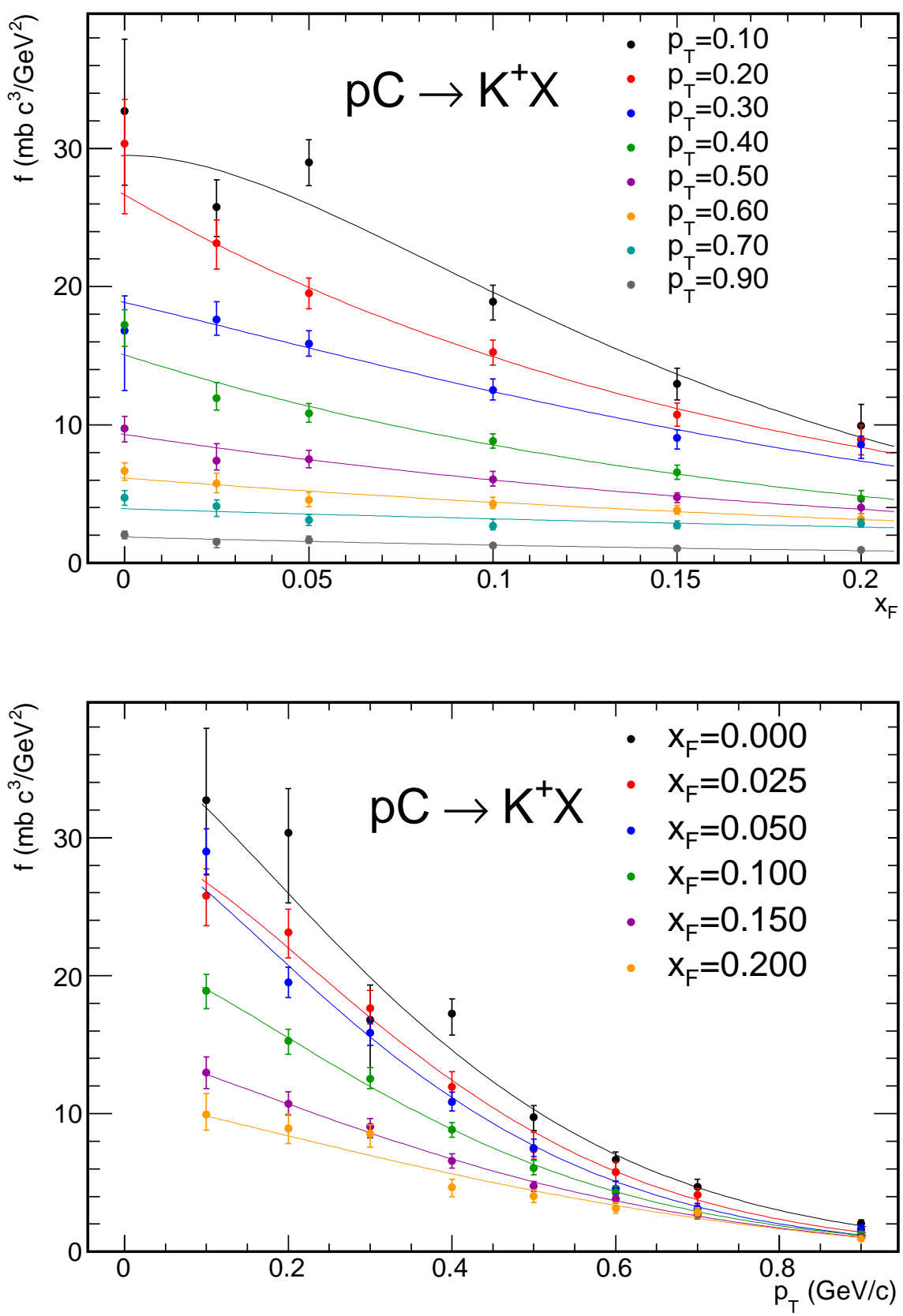

Figure 6.19: The figure shows the inclusive invariant cross section results for $\mathrm{K}^{+}$as a function of $x_{F}$ (top) and as a function of $p_{T}$ (bottom). The lines superimposed are only meant to guide the eyes. 

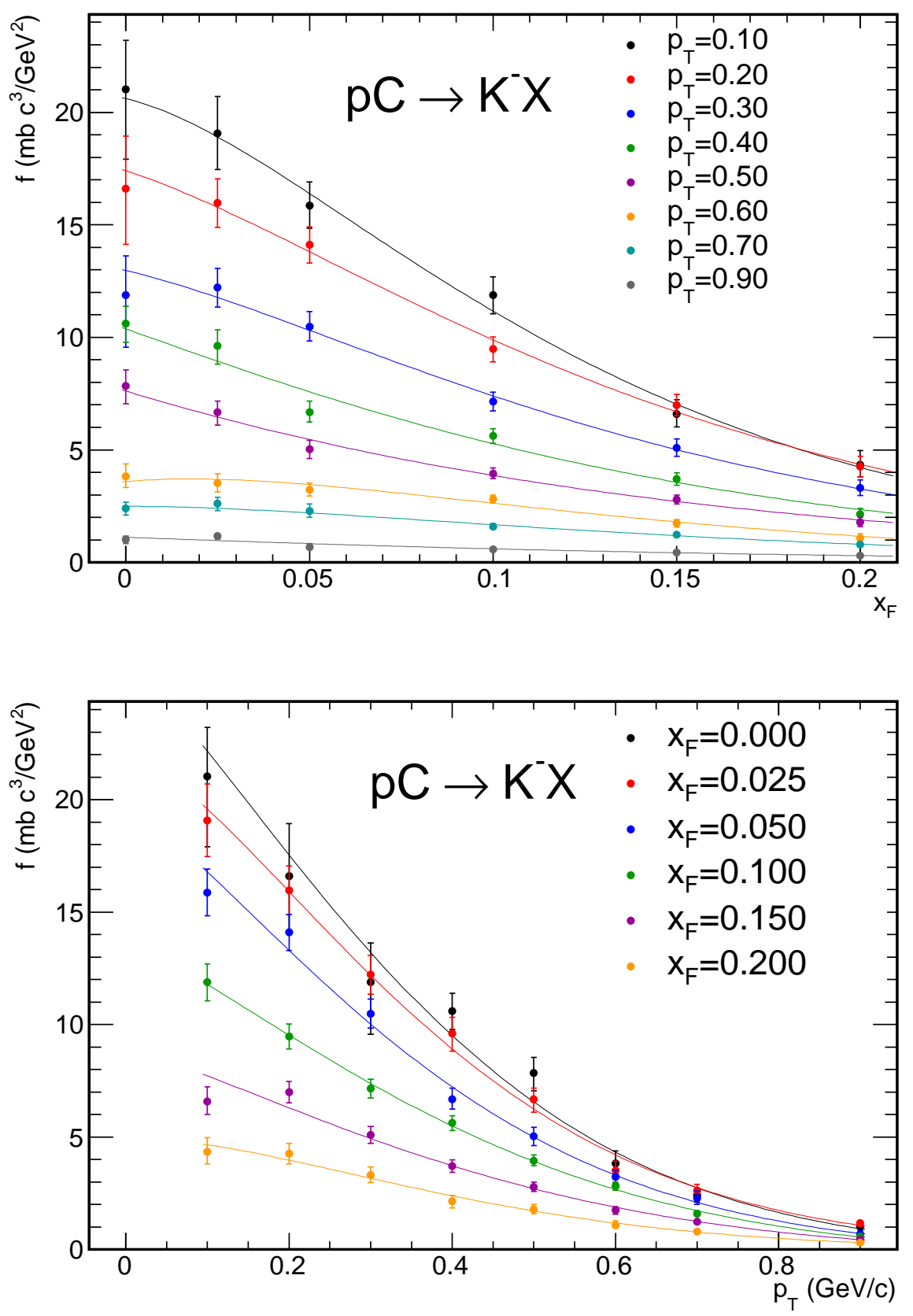

Figure 6.20: The figure shows the inclusive invariant cross section results for $\mathrm{K}^{-}$as a function of $x_{F}$ (top) and as a function of $p_{T}$ (bottom). The lines superimposed are only meant to guide the eyes. 

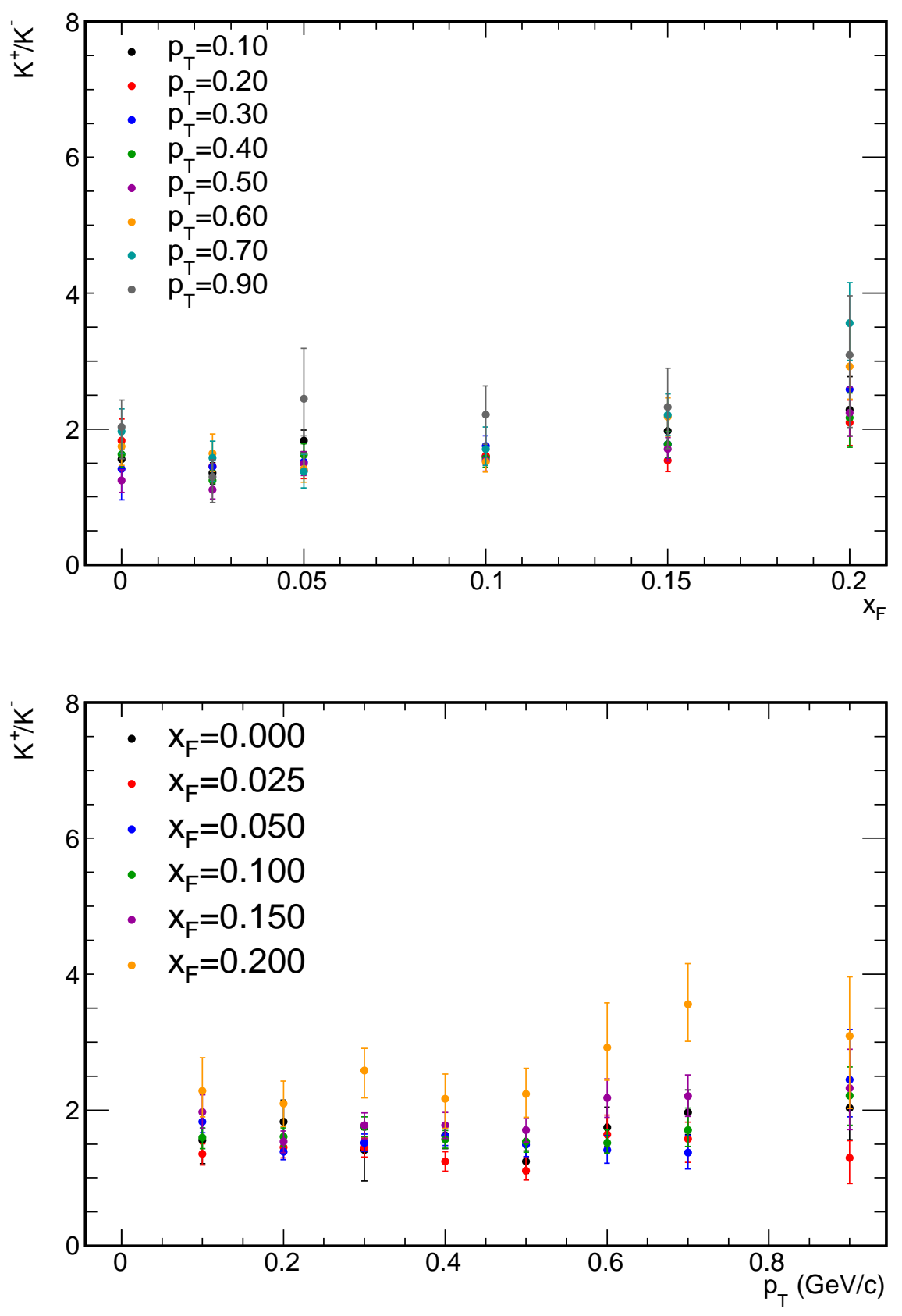

Figure 6.21: The plots show the ratio of $K^{+}$to the $K^{-}$production cross sections as a function of $x_{F}$ (top) and $p_{T}$ (bottom). 


\section{Chapter 7}

\section{Neutrino flux in MINOS and \\ comparison with the NA49 data}

This chapter presents a comparison of the NA49 kaon measurements described in chapter 6 with existing measurements and with the procedure that MINOS currently uses to evaluate the beam effects. The results for pions and kaons are summarised in section 7.1. Section 7.2 introduces the two other experiments which have previously measured kaon production cross sections or kaon ratios in $\mathrm{pC}$ interactions: there is a spread in proton beam energy, target thickness and $\left(x_{F}, p_{T}\right)$ covered by the different experiments which limits a direct comparison. The neutrino production in the NuMI beamline is presented in section 7.3. The Beam Working group in MINOS uses the Near Detector charged current selected spectrum to constrain the flux of neutrinos by introducing various weights to the $\mathrm{MC}$ to minimise the differences with the data spectrum. The comparison of the ratios of the secondary mesons as obtained in the MINOS tuned MC and from NA49 is presented in section 7.4. 

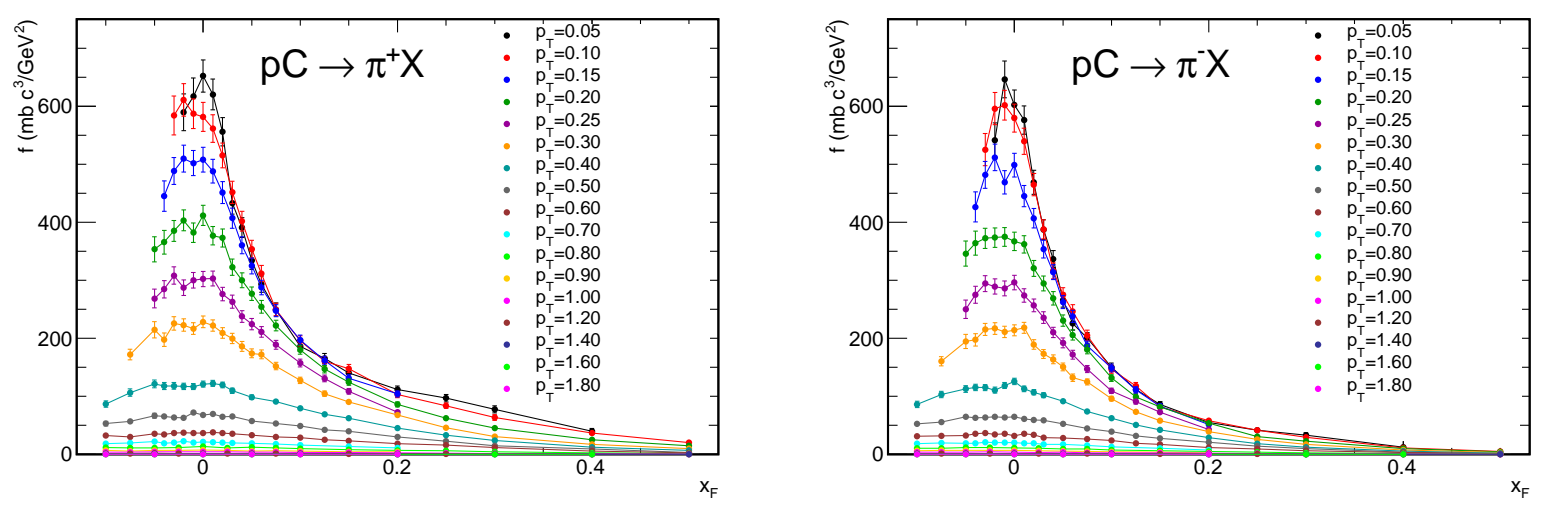

Figure 7.1: The pion inclusive invariant cross sections at fixed $p_{T}$ as a function of $x_{F}$. The plot on the left is for $\pi^{+}$, the plot on the right is for $\pi^{-}$. The plots are taken from [119] and the error bars include the systematic error summed in quadrature.

\subsection{Results from pC interactions in NA49}

\subsubsection{Inclusive production cross section of pions}

Results for the inclusive invariant production cross section of pions in $\mathrm{pC}$ interactions are given in [119]. In this thesis, when identifying kaons through a fit to the $d E / d x$ distributions, identification of pions is also performed. This is carried out in bins of $x_{F}$ which have been defined by assuming the kaon mass. Conversions from bins in $x_{F}$ to longitudinal momentum $p_{z}$ would be trivial. Nevertheless, due to the extremely low statistics of the kaons, the $\left(x_{F}\right.$, $\left.p_{T}\right)$ bins are too broad for an optimal description of the pion cross sections. Moreover, all the corrections would need to be re-evaluated in the new binning scheme. For these two reasons, the discussion of pion yields in this chapter will be based on the official NA49 pion production results [119] rather than the ones which could be obtained from the analysis in chapter 6 , as they are the best results available. Figure 7.1 shows the pion inclusive cross sections at fixed $p_{T}$ as a function of $x_{F}{ }^{1}$. The $3.8 \%$ systematic error quoted in [119] has been added in quadrature to the statistical error.

\footnotetext{
${ }^{1}$ Tables for the official NA49 results can be found at: https://spshadrons.web.cern.ch/spshadrons/pCdata.html\#pC_pion
} 

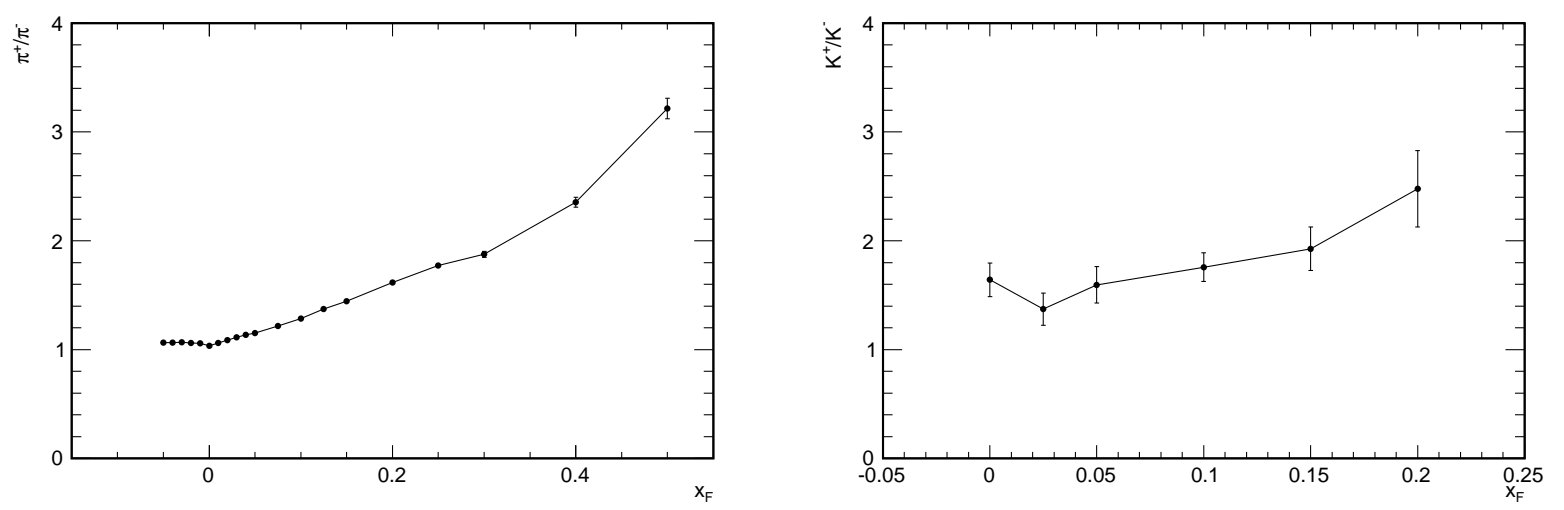

Figure 7.2: The plots show the $p_{T}$ integrated $\pi^{+} / \pi^{-}$ratio (left) as taken from [119] and the $\mathrm{K}^{+} / \mathrm{K}^{-} p_{T}$ integrated ratio (right) as a function of $x_{F}$.

\subsection{2 $\pi$ and $\mathrm{K} p_{T}$ integrated distributions}

Using the definition of the invariant double differential cross section $f$ in equation (6.9), it is possible to evaluate the $p_{T}$ integrated invariant cross sections (measured in $\mathrm{mb} \cdot c$ ) as:

$$
F=\int f d p_{T}^{2}
$$

The integrated yield distributions will be used to evaluate the ratios $\pi^{+} / \pi^{-}, \mathrm{K}^{+} / \mathrm{K}^{-}$and $\mathrm{K}^{ \pm} / \pi^{ \pm}$. The $p_{T}$ integration is performed analytically. An assumption of the underlying distribution of the differential cross section dependence on $p_{T}$ is necessary in the integration. For the pion cross section, the official results presented in [119] have been used. For the kaon cross section, the parametrisation used for the integration is given in equation (6.12).

Figure 7.2 shows the $p_{T}$ integrated $\pi^{+} / \pi^{-}$ratio (left) and the $p_{T}$ integrated $\mathrm{K}^{+} / \mathrm{K}^{-}$ ratio (right) as a function of $x_{F}$ (with either the $\pi$ mass assumption or the $\mathrm{K}$ one). More details about the shape of the meson ratios are given in section 7.4, but in anticipation, the increasing ratio as a function of $x_{F}$ has to do with the quark content in the p projectile. Figure 7.3 shows the average $p_{T}$ as a function of $x_{F}$ for $\pi^{+}$and $\pi^{-}$(left) and $\mathrm{K}^{+}$and $\mathrm{K}^{-}$ (right). 

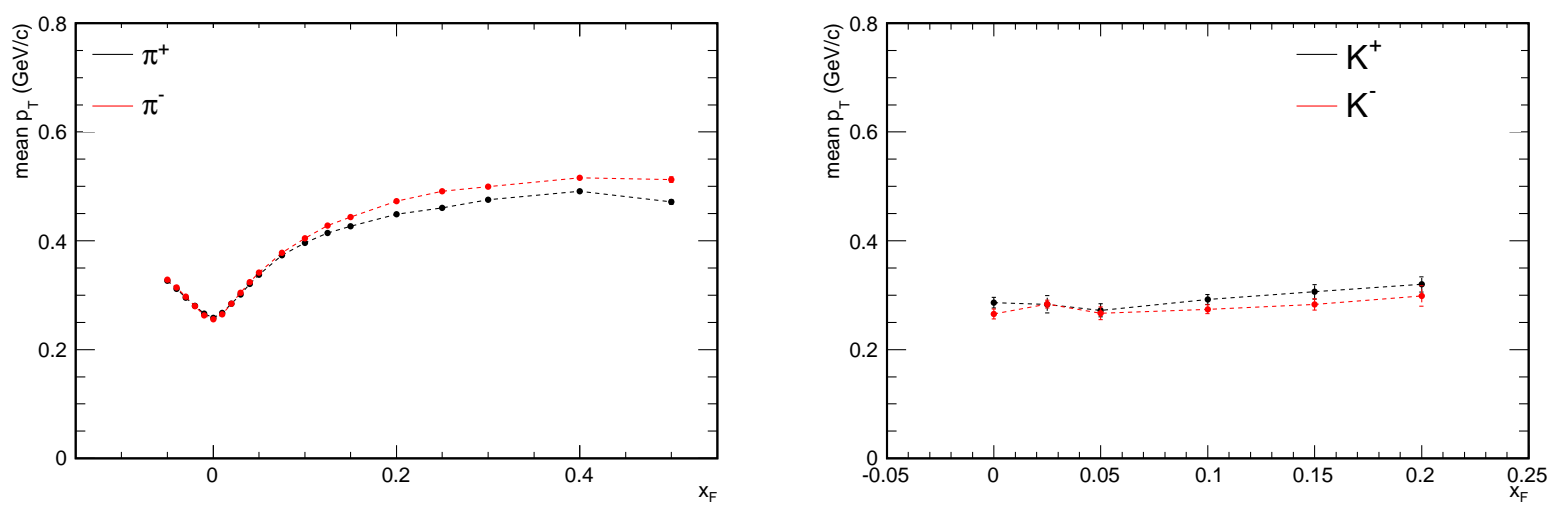

Figure 7.3: The plot on the left shows the average $p_{T}$ as a function of $x_{F}$ for $\pi^{+}$in black and $\pi^{-}$in red as taken from [119]. The plot on the right shows the average $p_{T}$ as a function of $x_{F}$ for $\mathrm{K}^{+}$in black and $\mathrm{K}^{-}$in red.

\subsubsection{Production of neutral kaons}

A direct measurement of the $\mathrm{K}_{S}^{0}$ yields cannot be performed using the $d E / d x$ discrimination technique $^{2}$. An indirect measurement can be obtained by weighting the number of $\mathrm{K}^{+}$and $\mathrm{K}^{-}$for their quark contents assuming $u_{v} / d_{v}=2$ and $u_{s}=\bar{u}_{s}=d_{s}=\bar{d}_{s}$ and $s_{s}=\bar{s}_{s}$, as described in [132]:

$$
N\left(\mathrm{~K}_{S}^{0}\right)=N\left(\mathrm{~K}_{L}^{0}\right)=\frac{N\left(\mathrm{~K}^{+}\right)+3 N\left(\mathrm{~K}^{-}\right)}{4} .
$$

Figure 7.4 shows the integrated $p_{T}$ cross sections for $\mathrm{K}^{+}$and $\mathrm{K}^{-}$(left) and the derived integrated cross section for $\mathrm{K}_{S}^{0}$ as function of $x_{F}$.

\subsection{Results from $\mathrm{pC}$ interactions from other experi- ments}

There is a lack of worldwide data of kaon inclusive production cross sections in pC interactions, which is the main motivation for the study in this thesis. Figure 7.5 shows the phase space in $\left(p_{z}, p_{T}\right)$ covered by experimental results on kaon production in pC. Only two experiments, in addition to NA49, have produced results. The different experiments

\footnotetext{
${ }^{2}$ The $\mathrm{K}_{S}^{0} V^{0}$ analysis has been performed for the pp data in [118] by studying the decay product in $\mathrm{K}_{S}^{0} \rightarrow \pi^{+} \pi^{-}$.
} 

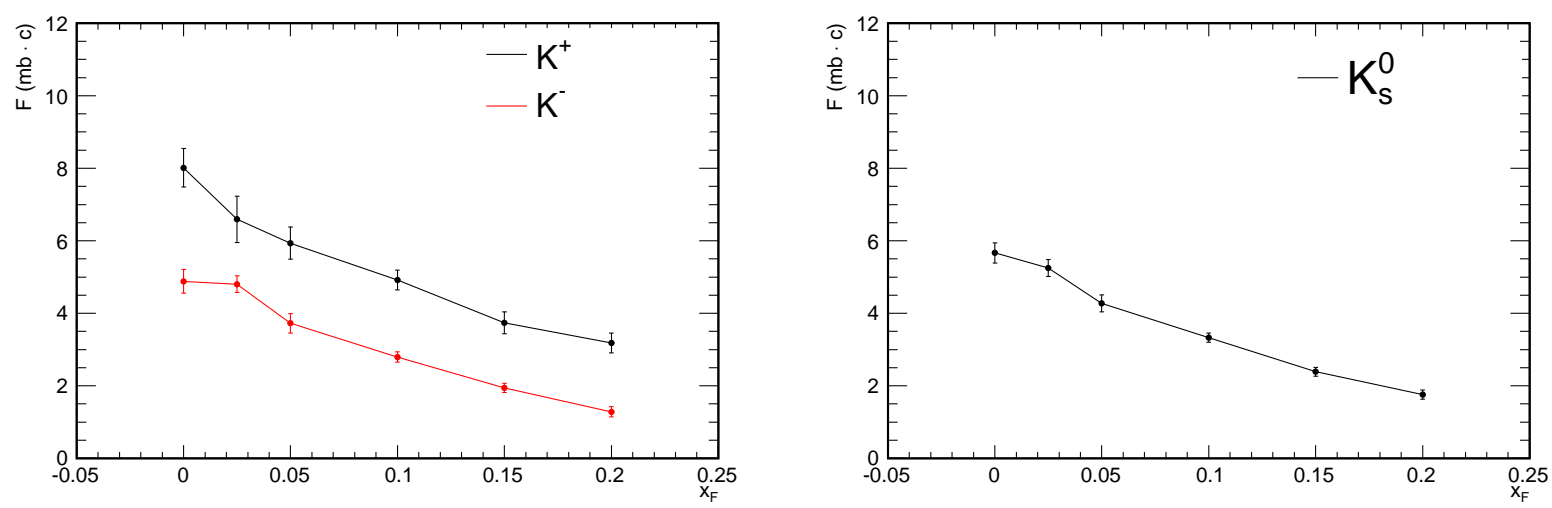

Figure 7.4: The figure shows the $p_{T}$ integrated cross section for $\mathrm{K}^{ \pm}$(left) and for $\mathrm{K}^{0}$ (right) as a function of $x_{F}$.

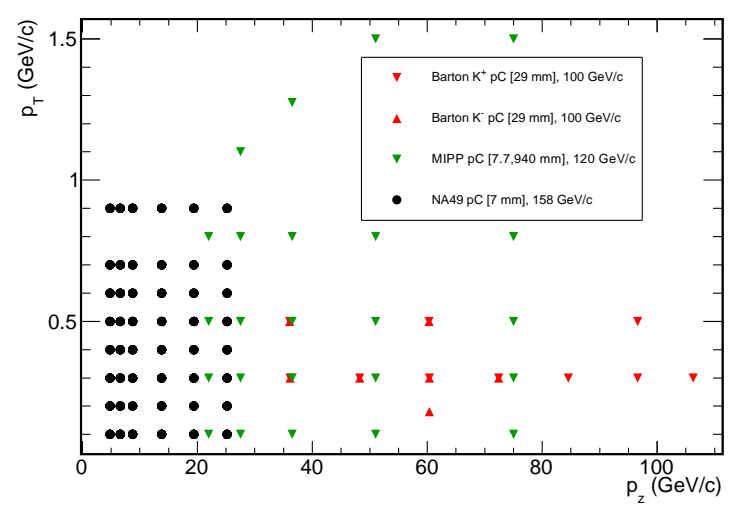

Figure 7.5: $\left(p_{z}, p_{T}\right)$ phase space covered by experimental results on kaon production in $\mathrm{pC}$ interactions. The results have been scaled according to $x_{F}$, normalising to the MINOS beam momentum of $120 \mathrm{GeV} / c$.

use different target thickness and beam momenta. Figure 7.5 shows $p_{z}$ after it has been corrected by scaling according to $x_{F}$, as explained in section 7.4.

The Fermilab Single Arm Spectrometer facility has published particle cross section ratios in 1983 [133] (Barton et al.). They used a beam of protons at $100 \mathrm{GeV} / c$. They scanned a range of momentum greater than $30 \mathrm{GeV}\left(x_{F}>0.3\right)$ and three $p_{T}$ values. Particle identification was achieved using Cerenkov counters. The target used had a thickness of $29 \mathrm{~mm}$, which is a factor $\approx 4.5$ larger than the thickness of the NA49 carbon target. Figure 7.6 shows the cross sections for $\mathrm{K}^{+}$(left) and $\mathrm{K}^{-}$(right) obtained in NA49 and in Barton et al. as a function of $x_{F}$ and for the same $p_{T}$. The superimposed line is the result of the fit 

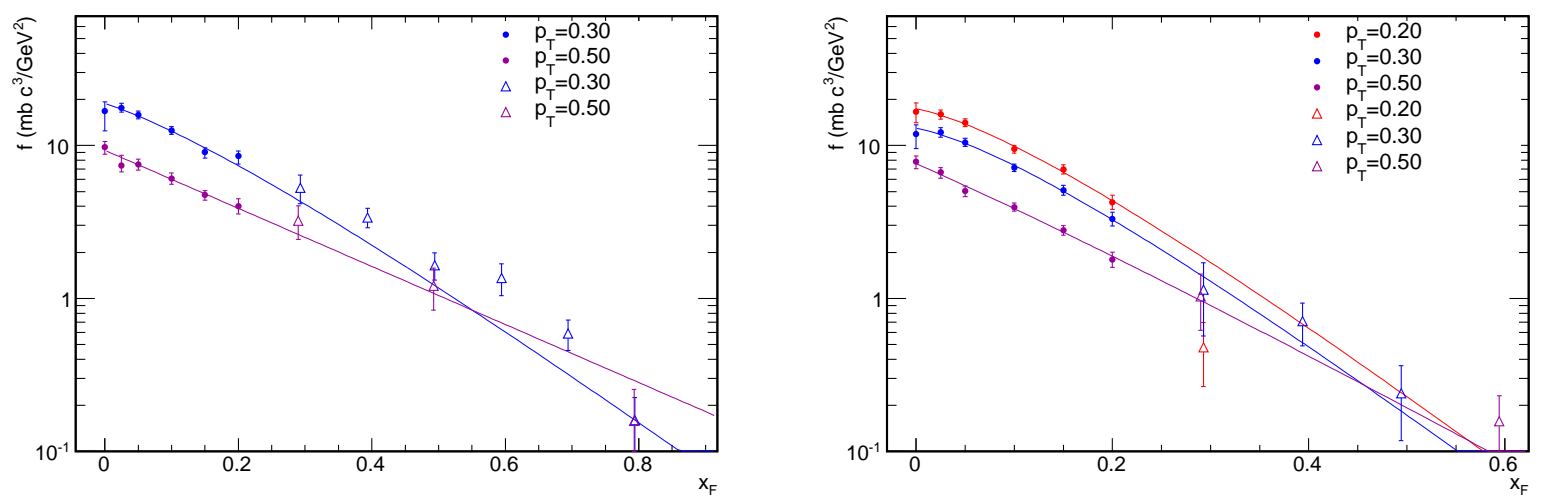

Figure 7.6: The figure shows the comparison of the kaon production cross sections as a function of $x_{F}$ in NA49 and in [133]. The plot on the left is for $\mathrm{K}^{+}$, the one on the right for $\mathrm{K}^{-}$. Only cross section at the same $p_{T}$ are shown. The filled circle is for NA49 results, the open triangle is for the results in Barton et al.

of the function in equation (6.11) only to the NA49 data. Given the different conditions of the data taking (centre of mass energy and target thickness) and the different regions of $x_{F}$ covered, there seems to be a general agreement between the two measurements.

The Main Injector Particle Production (MIPP) experiment uses the Main Injector beam of protons at Fermilab with momentum $120 \mathrm{GeV} / c$ (the same used by MINOS) to study hadron production on various targets. Particle identification is achieved using Ring Imaging Čerenkov detectors. The region of momentum studied is $p_{T}<2 \mathrm{GeV} / c$ and $p_{z}>20 \mathrm{GeV} / c$ and $p_{z}<90 \mathrm{GeV} / c$, which is roughly the same region studied by Barton et al. Official results have not been released, but there are two $\mathrm{PhD}$ theses available [134, 135]. They have studied two different target thickness, $7.7 \mathrm{~mm}$ (approximately the same thickness of the NA49 target) and $940 \mathrm{~mm}$ (the same target as used in MINOS). The results provided are not the absolute kaon cross sections, but kaon (and pion) ratios. A comparison of the results with NA49 will be presented in section 7.4.

The CERN NA61 [136] experiment is currently pursuing a physics program very similar to the NA49 one. Data from pC collisions with the proton beam at $31 \mathrm{GeV} / c$ momentum were recorded in 2007. The intent is to produce direct measurement of the hadron production using the exact same experimental conditions as the T2K long baseline experiment. 


\subsection{Rate of neutrinos in MINOS}

The NuMI beamline has been described in section 3.2. The major source of uncertainty in the NuMI beam simulation is given by the lack of knowledge of the hadron production off the target. The NuMI beamline is able to select mesons with different $\left(p_{z}, p_{T}\right)$ to obtain different neutrino spectra by moving the target position and changing the horn current. This unique feature of the beamline can be exploited to constrain the hadron production from the target from a joint fit to the data with the different settings. Figure 7.7 shows the $\left(p_{z}, p_{T}\right)$ of the parent $\pi^{+}$mesons which generate neutrino charged current interactions at the Near Detector in the different beam configurations. The rate of neutrinos corresponds to the convolution of the effects of the neutrino flux, the neutrino interaction cross section and the reconstruction efficiencies. Figure 7.8 shows the same phase space for the $\mathrm{K}^{+}$ parent mesons in the different beam configurations. Changing the horn current has a major impact on the $p_{T}$ of the selected mesons, while moving the target influences the $p_{z}$ of the mesons. Figure 7.9 shows the Near Detector true $\nu_{\mu}$ charged current spectrum in true neutrino energy separating the contribution of the various parent mesons (left). $\pi^{+}$ (in red) are more important for true neutrino energy around $30 \mathrm{GeV}$, while $\mathrm{K}^{+}$are the most important contributor for high energy neutrinos. The figure on the right represents the corresponding distribution for true neutral current events. The behaviour for neutral current events is similar. This is expected as the underlying $\nu_{\mu}$ flux is equivalent (the true neutral current spectrum has some small contribution from the $\bar{\nu}_{\mu}$ and $\nu_{e}$ in the beam which can be neglected) and the ratio between the neutral current and charged current cross sections is quite flat for the energies relevant to MINOS. Any correction to the neutrino flux derived from the charged current energy spectrum can be applied to the neutral current spectrum. 

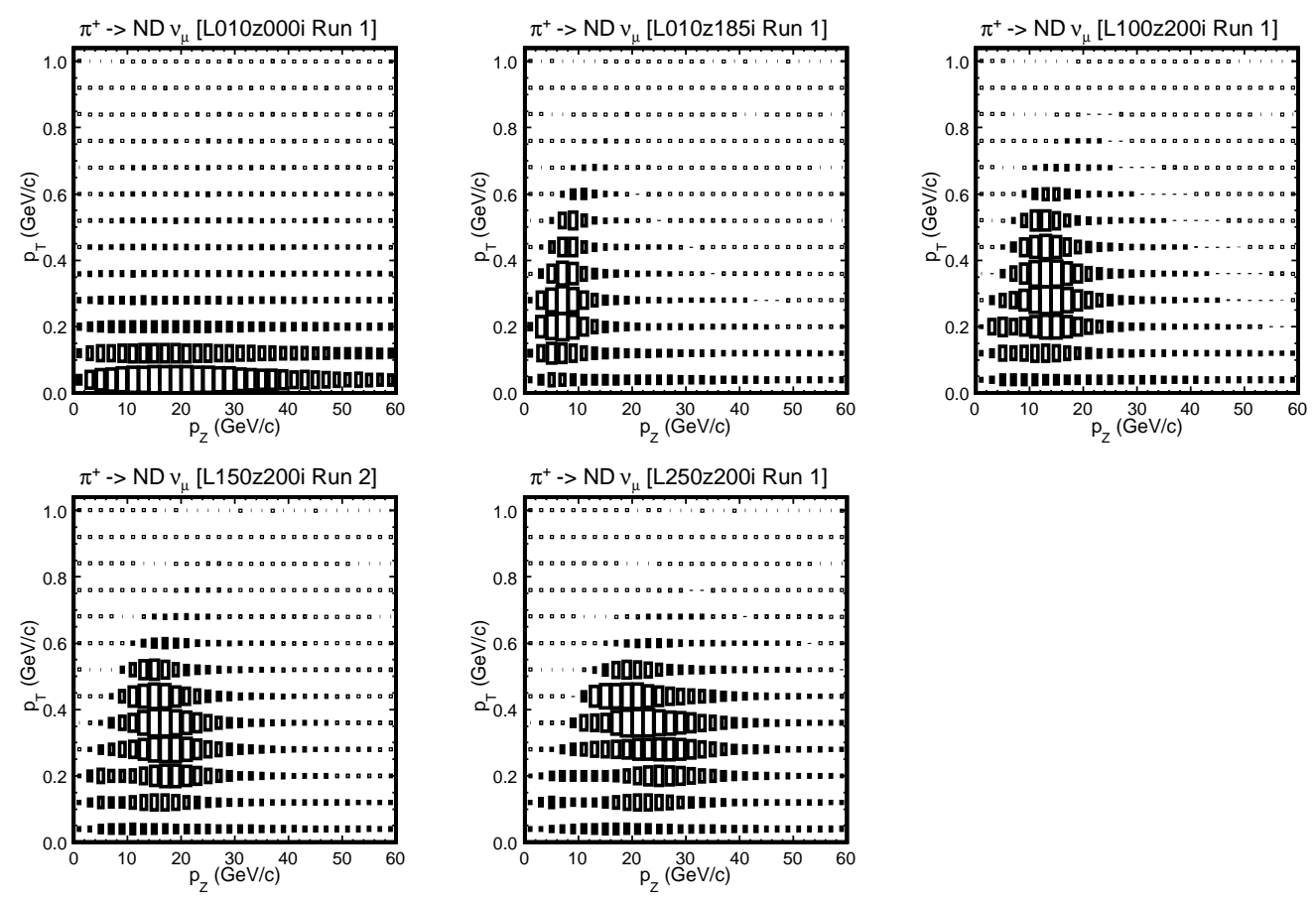

Figure 7.7: $\left(p_{z}, p_{T}\right)$ phase spaces for $\pi^{+}$which produce $\nu_{\mu}$ interactions in the Near Detector for various beam configurations. The number before the " $\mathrm{z}$ " in the beam configuration label refers to the distance (in $\mathrm{cm}$ ) between the target nominal position and its position inside the first horn; the number before the "i" refers to the horn current (in kA). The plot is taken from [137]. 

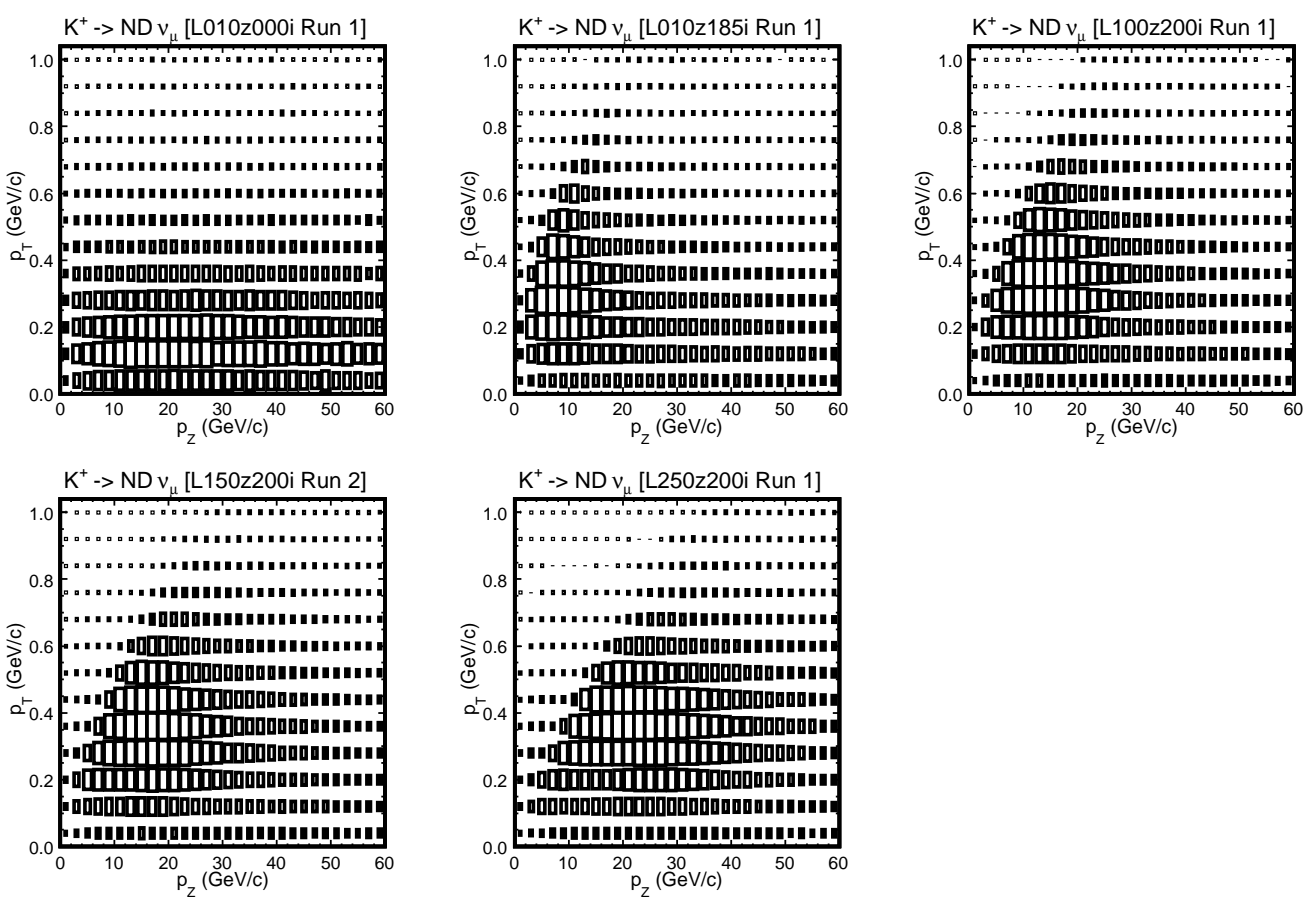

Figure 7.8: $\left(p_{z}, p_{T}\right)$ phase spaces for $\mathrm{K}^{+}$which produce $\nu_{\mu}$ interactions in the Near Detector for various beam configurations. The number before the " $\mathrm{z}$ " in the beam configuration label refers to the distance (in $\mathrm{cm}$ ) between the target nominal position and its position inside the first horn; the number before the "i" refers to the horn current (in kA). The plot is taken from [137].
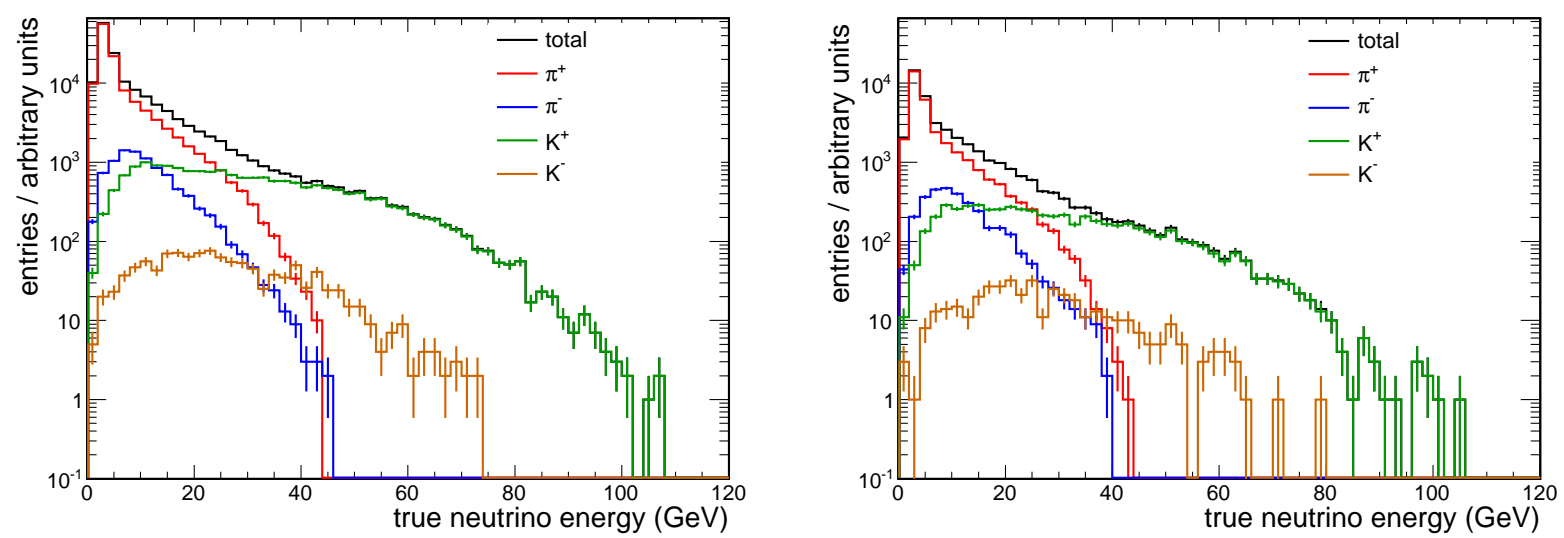

Figure 7.9: The plots show the neutrino events in MINOS Near Detector as obtained from the MC for true charge-current events (on the left) and true neutral-current events (on the right). The contributions from the different parent mesons are superimposed: the neutrino flux from $\pi^{+}$is shown in red, from $\pi^{-}$is shown in blue, from $\mathrm{K}^{+}$in green and from $\mathrm{K}^{-}$in brown. The fractional contribution is similar in both charged current and neutral current neutrino flux. 


\subsubsection{Tuning of the MINOS MC using Near Detector data}

The MC can be tuned in such a way that the disagreement between the selected charged current spectrum in data and $\mathrm{MC}$ is absorbed by a few parameters representing the hadron production weights, the uncertainties in the beamline (optic focusing, target position, target damage) and remaining reconstructed neutrino energy uncertainties (shower energy, cross section effects). This technique was originally studied in [138] and has been updated with the current reconstruction, MC version, target decay effects in [137]. The hadron production yield from the FLUKA $05^{3} \pi^{+}$simulation was parameterised as a function of $x_{F}$ and $p_{T}$ according to equation:

$$
f_{\pi^{+}}\left(x_{F}, p_{T}\right)=\frac{d^{2} N}{d x_{F} d p_{T}}=\left[A\left(x_{F}\right)+B\left(x_{F}\right) p_{T}\right] e^{-C\left(x_{F}\right) p_{T}^{3 / 2}}
$$

where $A, B$ and $C$ are functions of $x_{F}$. The function $f_{\pi^{+}}\left(x_{F}, p_{T}\right)$ in [138] describes the overall exponential fall of the cross section in $p_{T}$, which is shown in figure 7.1. The model does not assume perfect factorised scaling as a function of $x_{F}$ and $p_{T}$ and the invariance is broken by the dependence of $A, B$ and $C$ on $x_{F}$. When fitting the charged current selected MC spectrum to the MINOS data, six parameters are introduced to represent linear distortions in $x_{F}$ to the functions $A\left(x_{F}\right), B\left(x_{F}\right)$ and $C\left(x_{F}\right)$. The linearly distorted functions of $x_{F}$ are called $A^{\prime}\left(x_{F}\right), B^{\prime}\left(x_{F}\right)$ and $C^{\prime}\left(x_{F}\right)$. The hadron yield scaling weights $w$ are evaluated as:

$$
w_{\pi^{+}}\left(x_{F}, p_{T}\right)=\frac{f_{\pi^{+}}\left(x_{F}, p_{T}, A^{\prime}, B^{\prime}, C^{\prime}\right)}{f_{\pi^{+}}\left(x_{F}, p_{T}, A, B, C\right)} .
$$

The same weighting procedure is performed for $\mathrm{K}^{+}$with independent parameters. It is not possible to extract precise informations about $\pi^{-}, \mathrm{K}^{-}$given the low flux of $\bar{\nu}_{\mu}$ in the beam. The weights for negative mesons are obtained assuming a linear correlations with their corresponding positive meson weights.

\footnotetext{
${ }^{3}$ The beamline simulation uses the FLUKA08 package but the parameterisation of the hadron production yield has been done using FLUKA05. There is no expected difference as the update of the simulation covered different topics. The hadron production yield in FLUKA was modelled after data preceding the NA49 results, making the simulation completely independent from the NA49 data.
} 


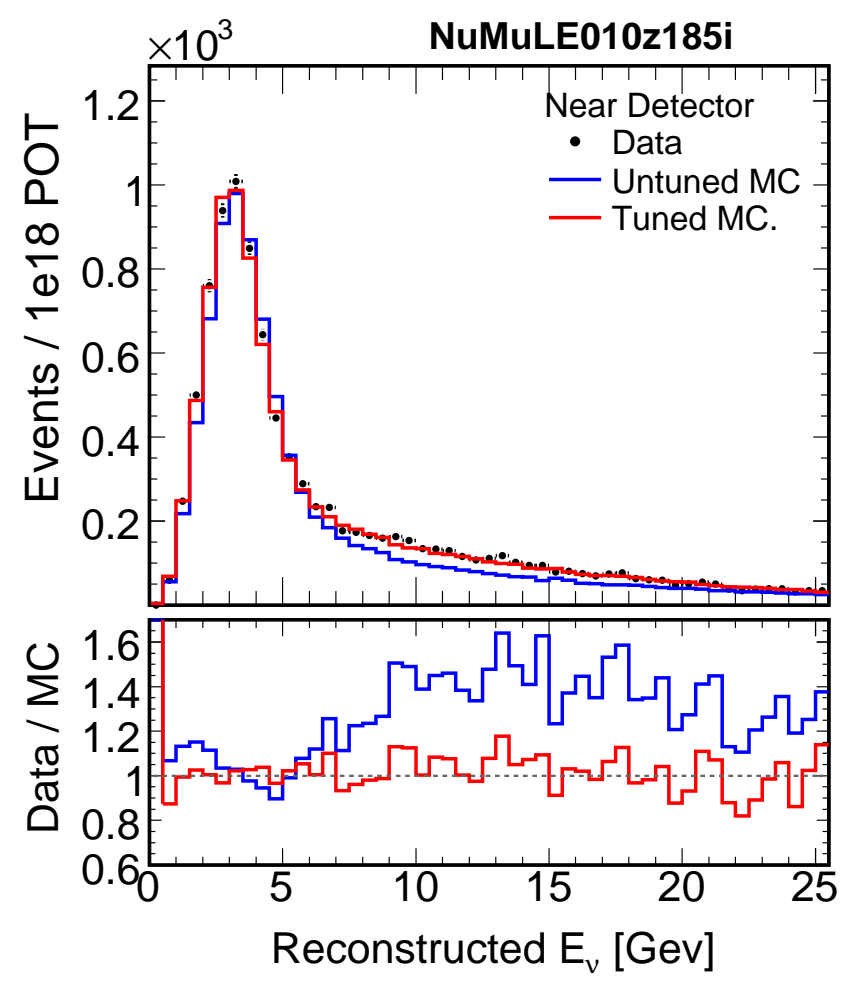

Figure 7.10: The plot at the top shows the effect of the MC tuning of the energy spectrum for charged current selected events in Run I at the Near Detector. Data are presented in black, the MC in blue and the tuned MC in red. The plot at the bottom shows the ratio between the data and the two MC in corresponding colours. The plot is taken from [137].

Figure 7.10 shows the effect of the MC tuning on the charged current selected spectrum at the Near Detector. Data are shown in black, the MC in blue and the tuned MC in red. The plot underneath shows the ratio of the data to the untuned (tuned) MC in blue (red).

\subsection{Hadron production in MINOS and NA49}

Despite the similarities in the production of secondary mesons from the target in MINOS and in NA49, the main differences are given by the different proton beam energy $(120 \mathrm{GeV} / c$ in NuMI versus $158 \mathrm{GeV} / c$ in NA49) and the different target thickness (940 mm in NuMI versus $7 \mathrm{~mm}$ in NA49) which causes considerably more secondary particle re-interaction in the NuMI target. None of those aspects are properly addressed in this section. The 
comparison between MINOS and NA49 is performed only on particle production ratios, not on the absolute cross sections, and so it is expected that some of the target reinteraction effects will cancel out. In the momentum range of interest to MINOS, it is expected that the interaction cross section of $\pi$ on deuterium (d), which is an isoscalar like carbon, is approximately equal for $\pi^{+}$and $\pi^{-}$(roughly constant at $45 \mathrm{mb}$ [12]). The interaction cross section of $\mathrm{K}^{+} \mathrm{d}$ is $35-40 \mathrm{mb}$ [12], while the interaction cross section of $\mathrm{K}^{-} \mathrm{d}$ is close to $40 \mathrm{mb}[12]$. In first approximation, it is possible to consider the $\mathrm{K}^{+} / \mathrm{K}^{-}$ratio not affected by target reinteraction, although a slight increase is expected. The $\mathrm{K}^{ \pm} / \pi^{ \pm}$is expected to change more due to the target reinteraction compared to $\mathrm{K}^{+} / \mathrm{K}^{-}$.

The MINOS beam reweighting uses constraints on the $\pi^{+} / \pi^{-}$, which are quadratically penalised to be within $5 \%$ of the official results of NA49. Figure 7.11 shows the $\pi^{+} / \pi^{-}$as a function of $p_{z}$ for the NA49 data in [119] in black dots, for different beam simulations and for the tuned simulation in red. Results for different simulations are shown, even though only FLUKA05 (in dotted red) has been officially implemented for MINOS. The increasing trend of the ratio with $p_{z}$ can be justified by the valence quark content in the proton: $\pi^{+}$contain $u_{v}$ quarks while $\pi^{-}$contain $d_{v}$ quarks. As the ratio $u_{v} / d_{v} \sim 2$, the $\pi^{+} / \pi^{-}$is expected to increase with $p_{z}$. When superimposing the results obtained in NA49, the different beam momenta $p_{\text {beam }}^{\mathrm{NA} 49}$ and $p_{\text {beam }}^{\mathrm{MINOS}}$ are corrected for by scaling according to $x_{F}$, as it is assumed that the cross section is only a function of $x_{F}$ and $p_{T}$. Given a NA49 data point with $x_{F}=f\left(p_{T}, p_{\text {beam }}^{\text {NA49 }}, p_{z}^{\text {NA49 }}\right)$, it is possible to find the corresponding longitudinal momentum at the MINOS beam energy $p_{z}^{\mathrm{MINOS}}$ such that $x_{F}$ is the same, where $x_{F}=f\left(p_{T}, p_{\text {beam }}^{\text {MINOS }}, p_{z}^{\mathrm{MINOS}}\right)$.

In the beam reweighting, the results of the kaons obtained in this thesis have not been used yet. This leaves the MINOS best fit and the NA49 results completely independent from each other. The $\mathrm{K}^{+} / \mathrm{K}^{-}$and the $\mathrm{K}^{ \pm} / \pi^{ \pm}$in the MINOS fit have been quadratically penalised to be within $20 \%$ of the FLUKA prediction. Figure 7.12 shows the $\mathrm{K}^{+} / \mathrm{K}^{-}$ behaviour as a function of $p_{z}$. The steep rise to high values of the ratio is justified by the ratio $u_{v} / \bar{u}_{s}$ from the quark constituents of the protons. The production of $\mathrm{K}^{+} \Lambda^{0}$ couples 


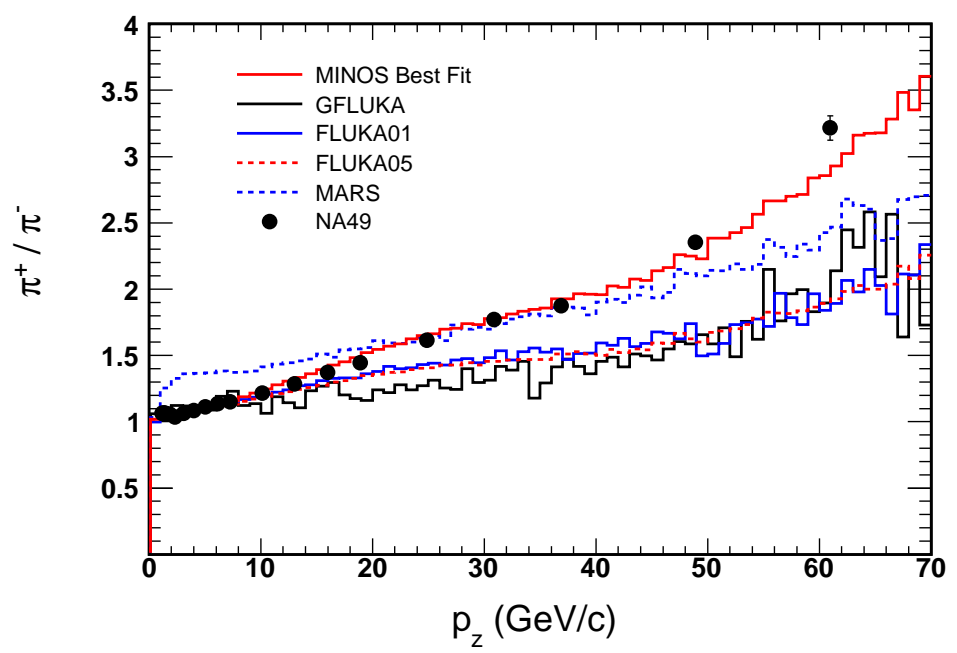

Figure 7.11: The plot shows the $\pi^{+} / \pi^{-}$as a function of $p_{z}$ for different MC simulations (but only FLUKA05 in dotted red has been used in the official MINOS beam simulation), for the beam reweighting best fit in red and for NA49 data in black.

in addition to $\mathrm{K}^{+} \mathrm{K}^{-}$ones will favour and higher $\mathrm{K}^{+} / \mathrm{K}^{-}$ratio. The NA49 data obtained in this thesis are shown as black dots, the FLUKA05 prediction is in dotted red and the MINOS reweighting best fit is in red. Figure 7.13 shows the ratio of the $\mathrm{K}^{+} / \pi^{+}$(left) and $\mathrm{K}^{-} / \pi^{-}$(right) behaviours as a function of $p_{z}$. The positive ratio is quite flat in the simulation and it is determined by the $\bar{s}_{s} / \bar{d}_{s}$ sea quark content in the proton. The $\mathrm{K}^{-} / \pi^{-}$ ratio decreases with $p_{z}$ due to the $s_{s} / d_{v}$ ratio. The pion $p_{T}$ integrated cross section NA49 data points have been linearly interpolated to provide coverage in $p_{z}$ and to obtain the $\mathrm{K}^{ \pm} / \pi^{ \pm}$data points. This introduces a further uncertainty on the $\mathrm{K}^{ \pm} / \pi^{ \pm}$values which are shown only for a qualitative comparison. Nevertheless, the results agree quite well with the $\mathrm{MC}$ expectation, with the exception of the highest $p_{z}$ point of the $\mathrm{K}^{+} / \pi^{+}$ratio, which is much higher for the NA49 data compared to the FLUKA05 simulation. The errors on the mesons production have been assumed to be uncorrelated in the evaluation of the meson ratio error as they are mainly statistical. 


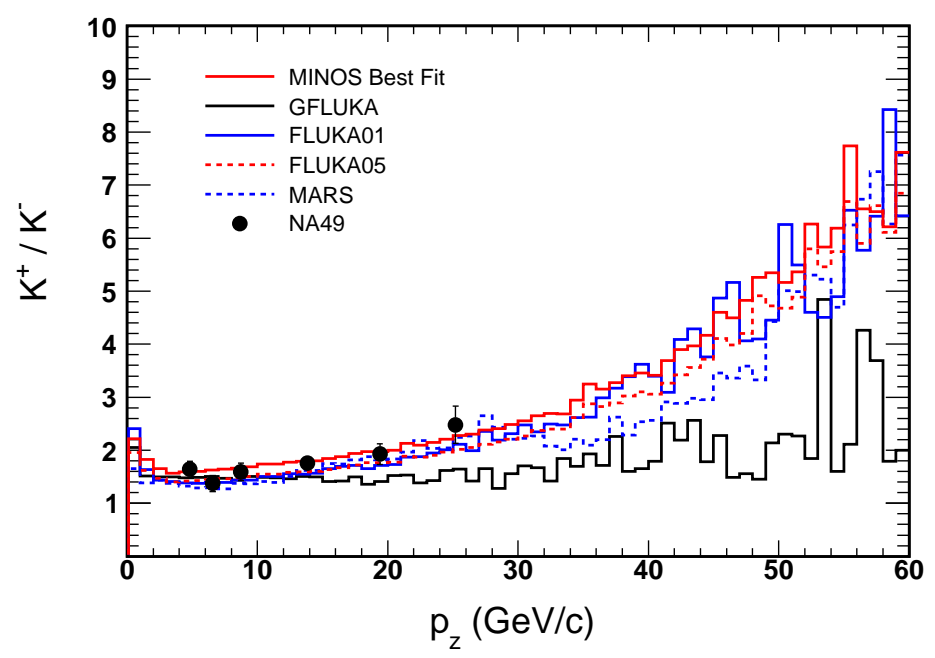

Figure 7.12: The plot shows the $\mathrm{K}^{+} / \mathrm{K}^{-}$as a function of $p_{z}$ for different $\mathrm{MC}$ simulations (but only FLUKA05 in dotted red has been used in the official MINOS beam simulation), for the beam reweighting best fit in red and as obtained in this thesis for NA49 data in black.
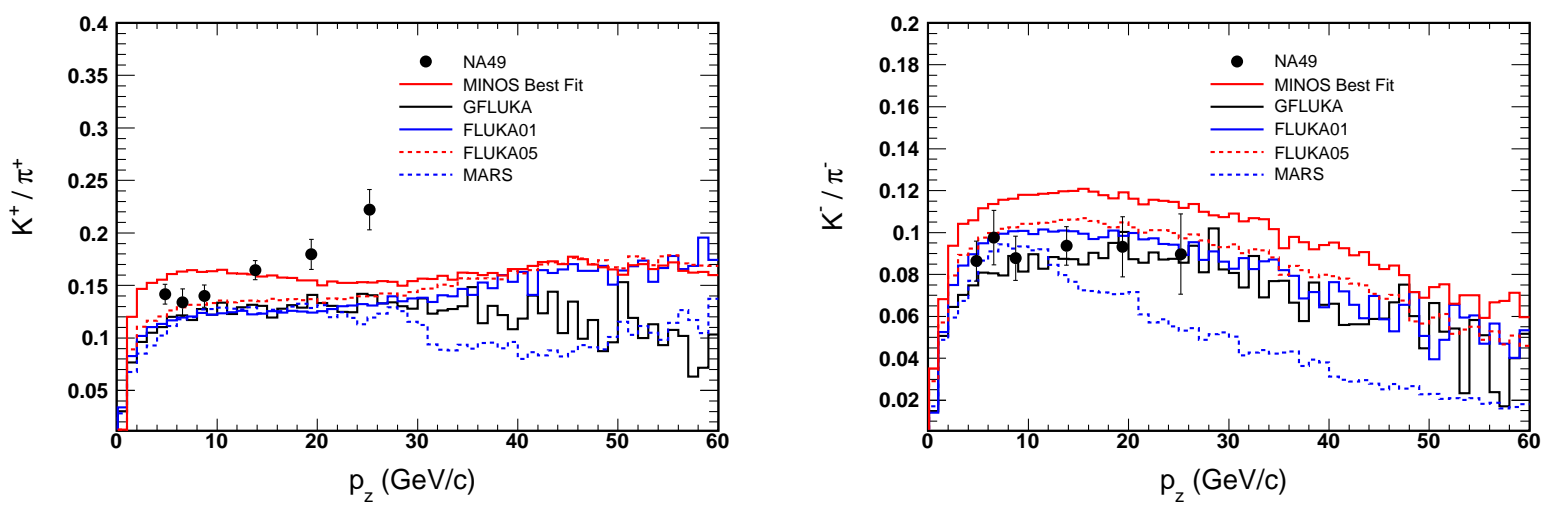

Figure 7.13: The plot shows the $\mathrm{K}^{+} / \pi^{+}$(left) and $\mathrm{K}^{-} / \pi^{-}$(right) as a function of $p_{z}$ for different MC simulations (only FLUKA05 in dotted red has been used in the official MINOS beam simulation), for the beam reweighting best fit in red and as obtained in this thesis for NA49 data in black. 


\subsubsection{Comparison of particle ratios for different $p_{T}$ ranges}

As previously seen, the $p_{T}$ integrated ratio in NA49 is used to constrain the MINOS fit. Studies were performed [138] and showed that the MINOS fit was close to the NA49 pion data ratio even before introducing any constraint. All the available MC expectation produce a lower ratio of the pions at higher $p_{z}$. MC studies in [139] have shown that the pion ratio remains approximately constant with a simulated thin target and it was decided to adopt the NA49 data constraint for the pion ratio. Figure 7.14 shows the ratio of $\pi^{+} / \pi^{-}$as a function of $p_{z}$ for different $p_{T}$ ranges: the FLUKA05 simulation is in dashed red, the MINOS best fit is in red and the NA49 data are black dots. The NA49 data agree remarkably well to the MINOS fit results, better than when compared to the FLUKA05 simulation for all $p_{T}$ ranges. The constraint on the integrated $p_{T}$ pion ratio has been preliminary applied to the MINOS fit, introducing a bias in the comparison of figure 7.14.

Figure 7.15 shows the $\mathrm{K}^{+} / \mathrm{K}^{-}$ratio as a function of $p_{z}$ for the FLUKA05 simulation in dashed red, for the MINOS best fit in red, for the NA49 data in black dots, for the Barton et al. results in red dots and for the MIPP results in blue and green dots. In the case of the Barton et al. data, scaling for $x_{F}$ has been performed to keep into account the different beam energy. The NA49 are in the region where statistically the kaons are more abundant and the errors are quite small compared to the other experiments. Nevertheless, the high $p_{z}$ data are important to the neutrino production as kaons are the only contributor to the neutrinos at higher energies. The $\mathrm{K}^{+} / \mathrm{K}^{-}$ratio at low $p_{T}$ seems slightly overestimated in the $\mathrm{MC}$, while it is underestimated at higher $p_{T}$. Nevertheless, given the limited data available and the uncertainties, the MINOS simulation is quite similar to the data.

Figure 7.16 shows the ratio of $\mathrm{K}^{+} / \pi^{+}$as a function of $p_{z}$ for different $p_{T}$ ranges. The agreement between data and the simulation in general is quite good, but the NA49 data seems to be slightly below the MC expectation. On the contrary, the MIPP data at high $p_{z}$ are all above the $\mathrm{MC}$.

The ratio of $\mathrm{K}^{-} / \pi^{-}$as a function of $p_{z}$ for different $p_{T}$ ranges is shown in figure 7.17. 

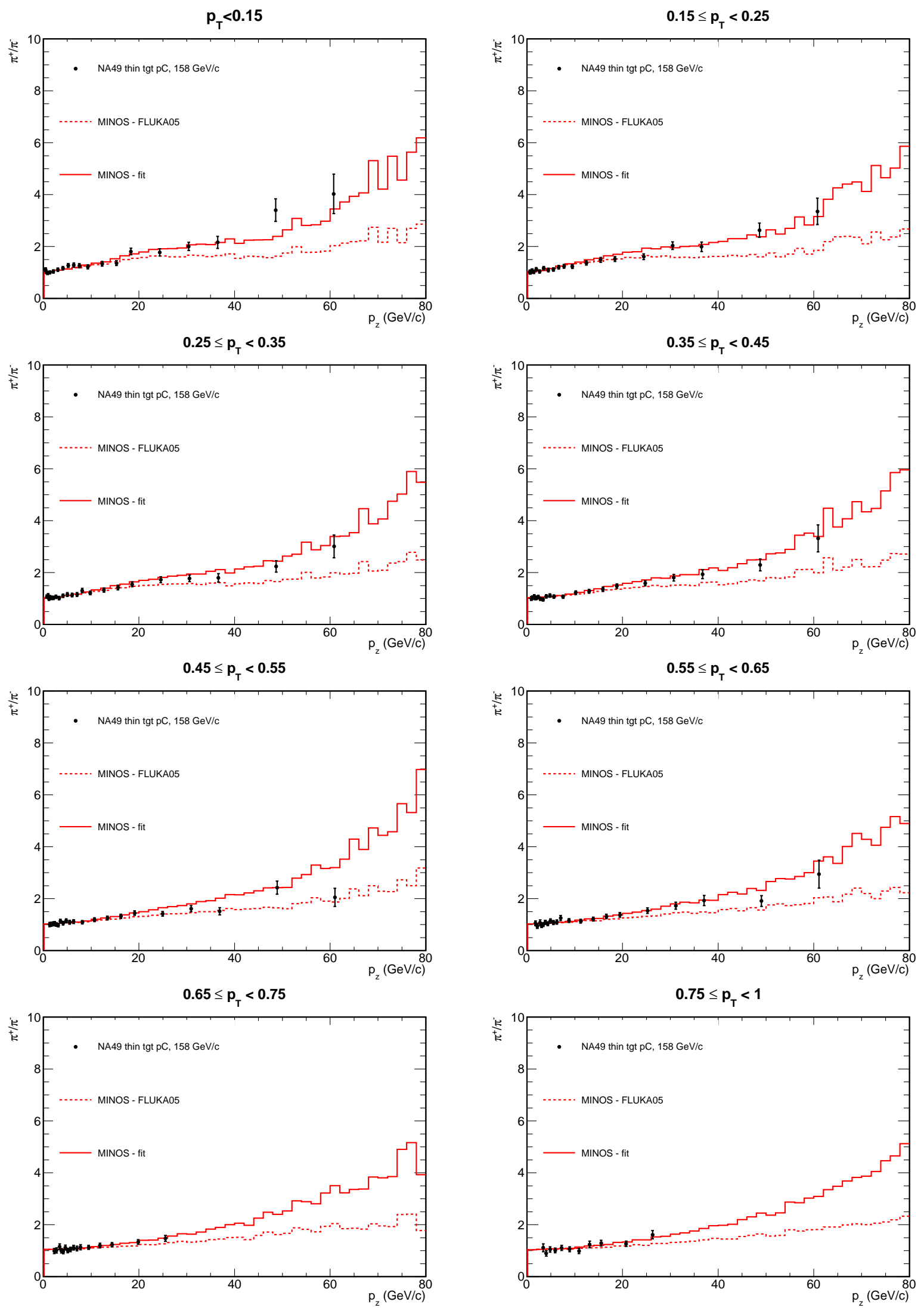

Figure 7.14: Comparison of $\pi^{+} / \pi^{-}$as a function of $p_{z}$ for different $p_{T}$ ranges. The solid red line represents the MINOS best fit, the dashed red line is FLUKA05 prediction and the black dots represent the NA49 results in [119]. The $p_{T}$ ranges are expressed in GeV/c. 

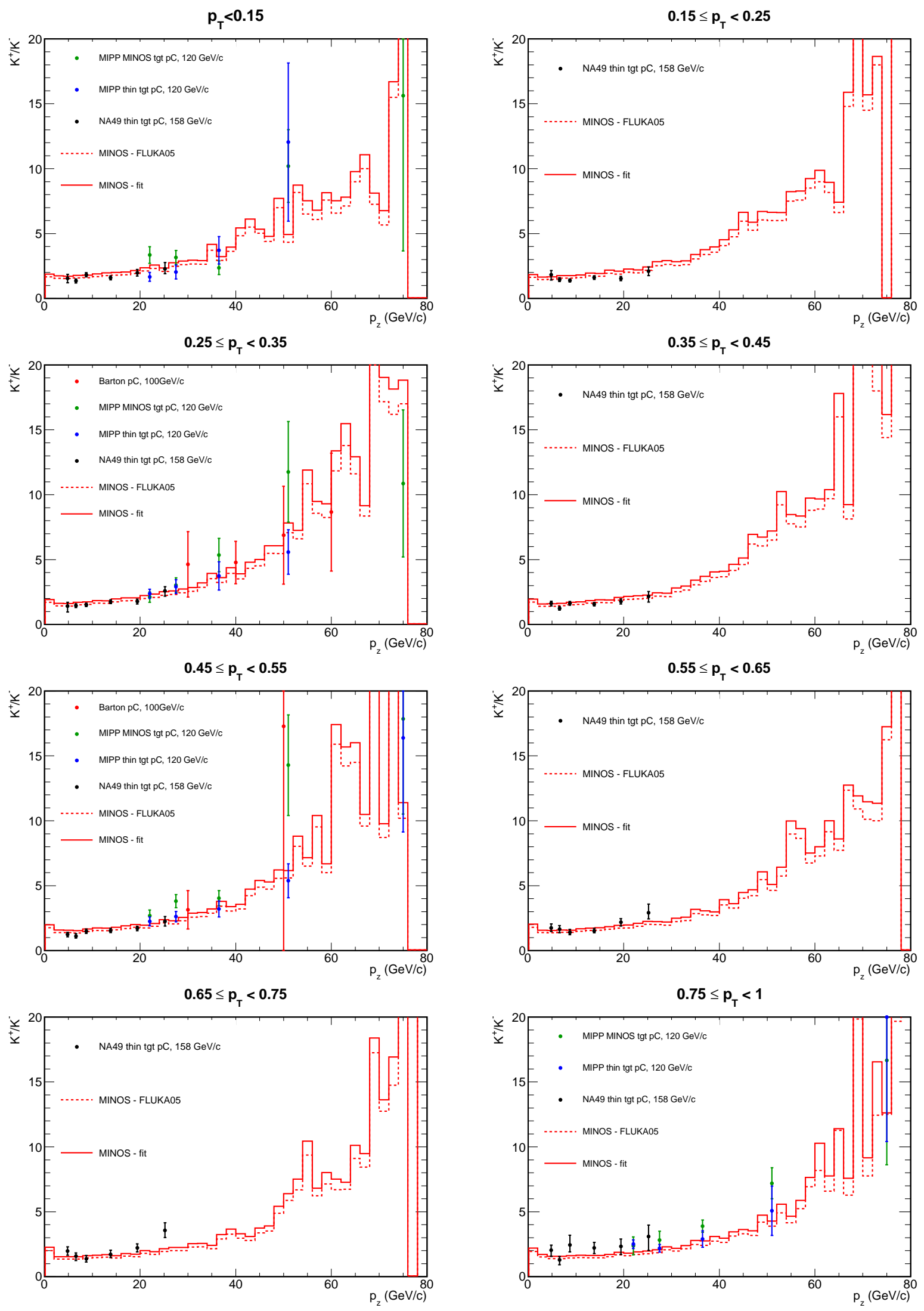

Figure 7.15: Comparison of $\mathrm{K}^{+} / \mathrm{K}^{-}$as a function of $p_{z}$ for different $p_{T}$ ranges. The solid red line represents the MINOS best fit, the dashed red line is FLUKA05 prediction and the black dots represent the NA49 results in this thesis. The MIPP results for the thin target are in blue and the ones for the MINOS target in green. 
The NA49 measurements are consistent with the MC expectations.

\subsubsection{Comparison of particle ratios for different $p_{z}$ ranges}

The comparison between the pion ratios as a function of $p_{T}$ in different $p_{z}$ bins was studied in [140]. The MINOS fit was found to be in agreement with the NA49 points, while the agreement with FLUKA05 was worse. Figure 7.18 shows the ratio $\mathrm{K}^{+} / \mathrm{K}^{-}$as a function of $p_{T}$ for different $p_{z}$ ranges. The MINOS fit and the FLUKA05 prediction are quite similar, and they are in disagreement with the NA49 data at high $p_{T}$. The $\mathrm{K}^{+} / \mathrm{K}^{-}$behaviour in the NA49 data is nearly flat in $p_{T}$, while the MINOS prediction has higher ratios at high $p_{T}$. For the plot in the $p_{z}$ range between 16 and $23 \mathrm{GeV} / c$, the MIPP data are superimposed: they agree with the NA49 data quite well.

Figures 7.19 and 7.20 show the ratios $\mathrm{K}^{+} / \pi^{+}$and $\mathrm{K}^{-} / \pi^{-}$as a function of $p_{T}$ in different $p_{z}$ bins. In both the cases the NA49 data show increasing meson ratios with increasing $p_{T}$, while the MINOS best fit and the FLUKA05 prediction show nearly flat ratios. The MIPP data overlapping in one $p_{z}$ bin agree well with the NA49 data.

\subsection{Conclusion}

The NA49 data extracted in this thesis, despite being in part of the phase space which is not critical for the neutrino production, represent a dramatic improvement on the worldwide kaon production data in $\mathrm{pC}$ interactions. An investigation of the effects of the different beam momentum and target thickness is necessary to use the NA49 data in the MINOS fits. Nevertheless, the consistency between the MIPP data with two different target thickness suggests that the particle ratios are not changed much by the effect of the reinteraction in the target. In general, MIPP data for the MINOS target are higher than the MIPP data for the thin target, as expected by the interaction cross sections of the mesons. While the MINOS fit is in quite good agreement with the NA49 meson ratios as a function of $p_{z}$, some discrepancies are found as a function of $p_{T}$, suggesting some uncertainty in the $p_{T}$ 

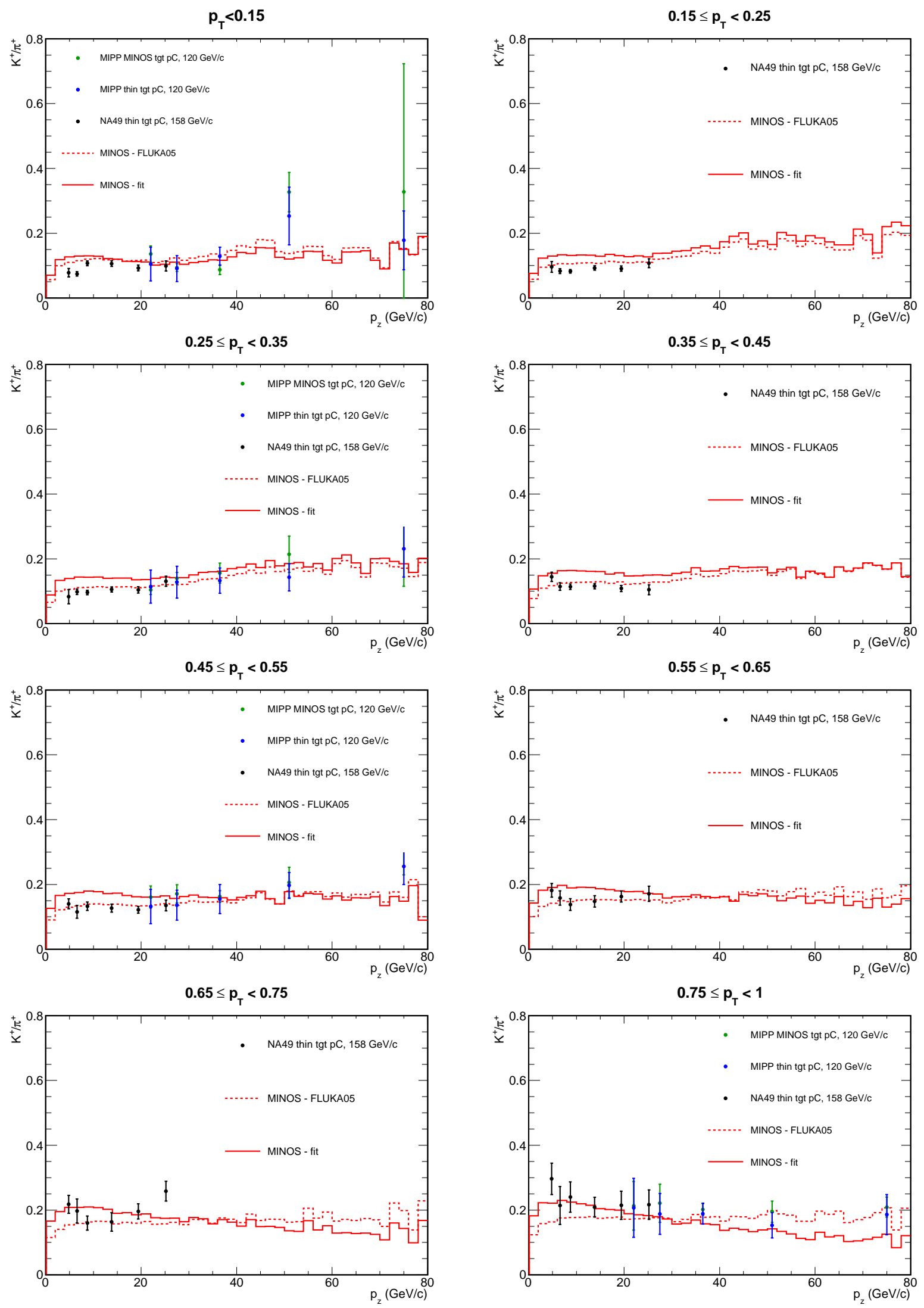

Figure 7.16: Comparison of $\mathrm{K}^{+} / \pi^{+}$as a function of $p_{z}$ for different $p_{T}$ ranges. The solid red line represents the MINOS best fit, the dashed red line is FLUKA05 prediction and the black dots represent the NA49 results in this thesis. The MIPP results for the thin target are in blue and the ones for the MINOS target in green. 

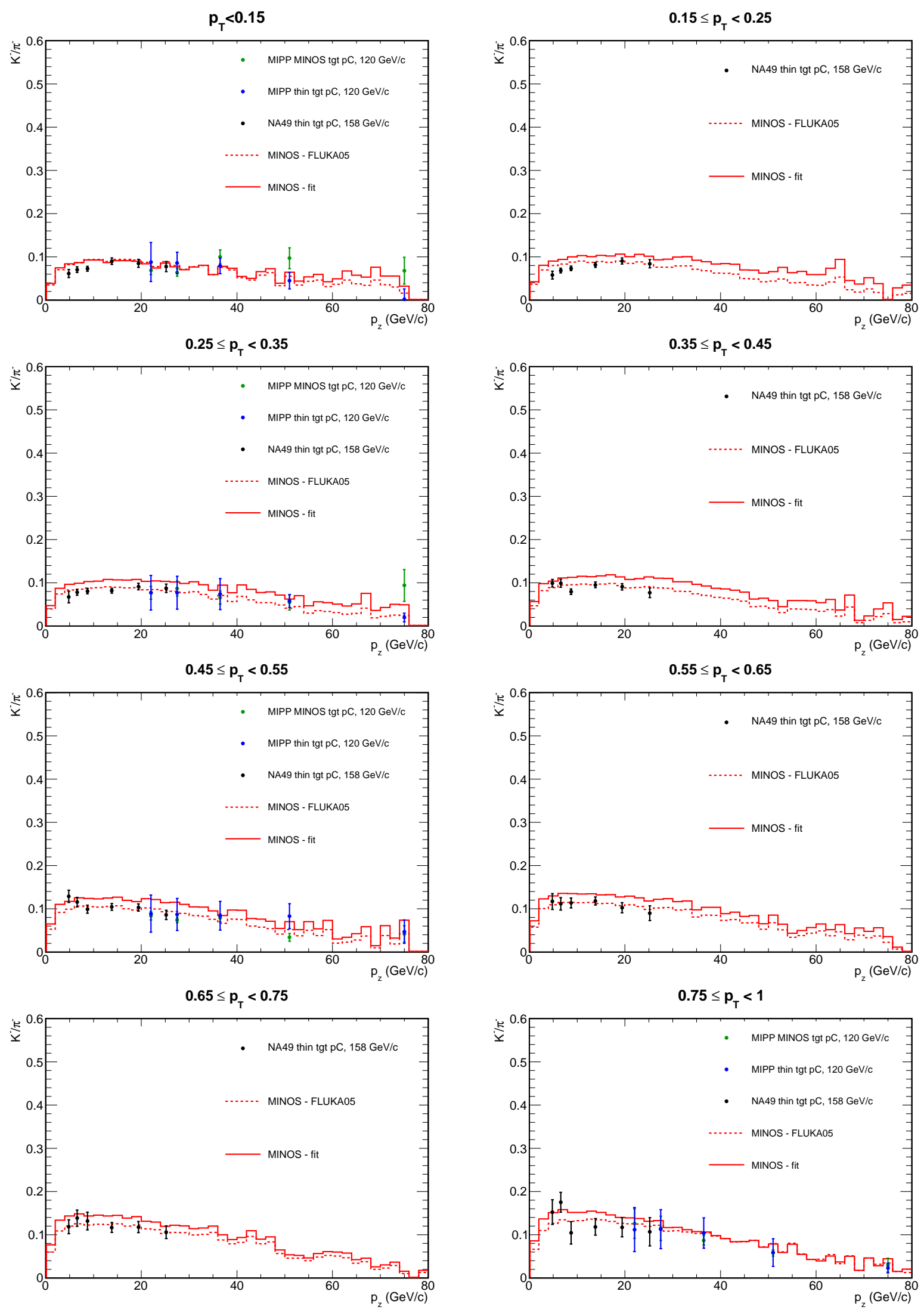

Figure 7.17: Comparison of $\mathrm{K}^{-} / \pi^{-}$as a function of $p_{z}$ for different $p_{T}$ ranges. The solid red line represents the MINOS best fit, the dashed red line is FLUKA05 prediction and the black dots represent the NA49 results in this thesis. The MIPP results for the thin target are in blue and the ones for the MINOS target in green. 

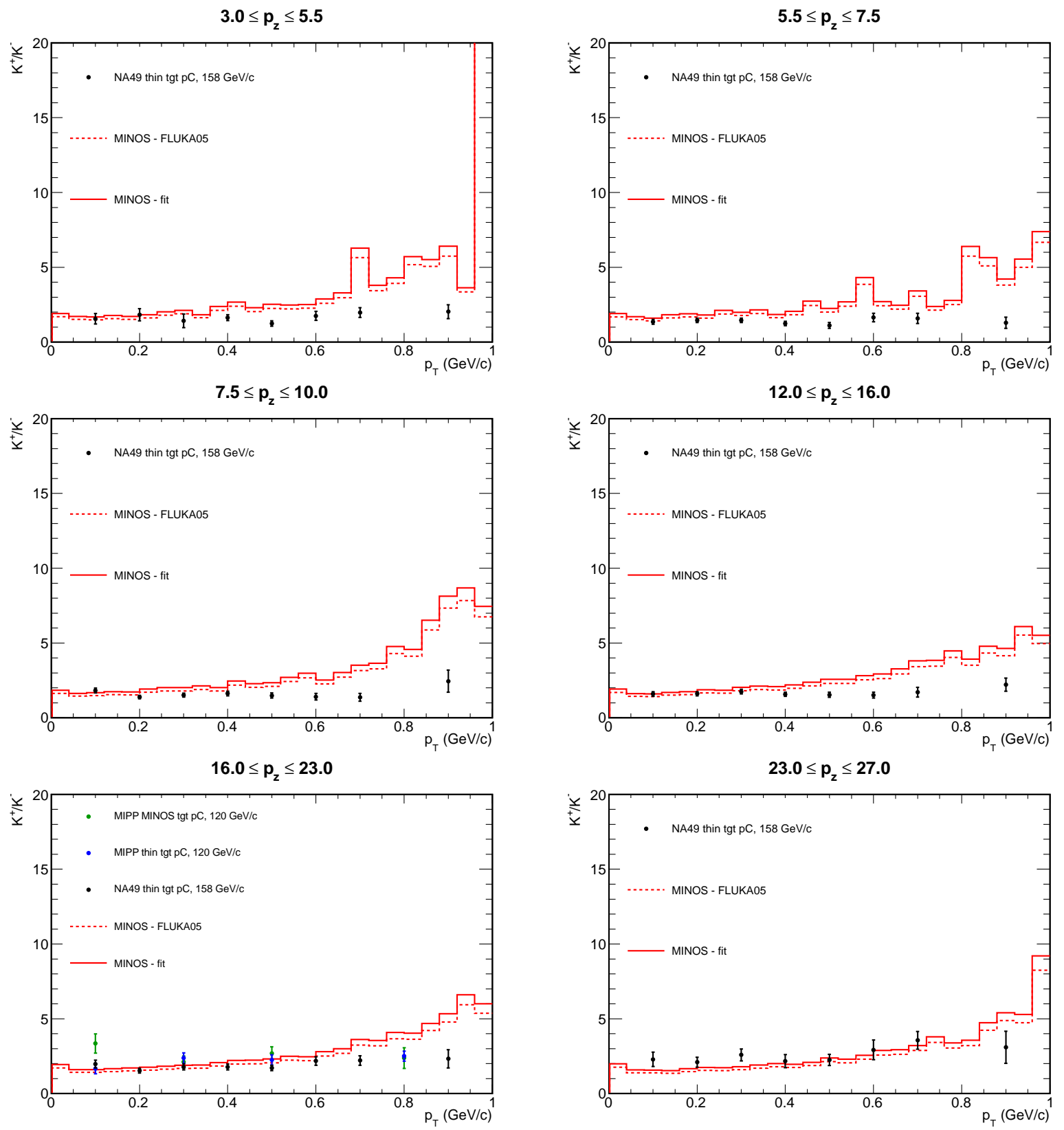

Figure 7.18: Comparison of $\mathrm{K}^{+} / \mathrm{K}^{-}$as a function of $p_{T}$ for different $p_{z}$ ranges. The solid red line represents the MINOS best fit, the dashed red line is FLUKA05 prediction and the black dots represent the NA49 results in this thesis. The MIPP results for the thin target are in blue and the ones for the MINOS target in green. The $p_{z}$ ranges are expressed in $\mathrm{GeV} / c$. 

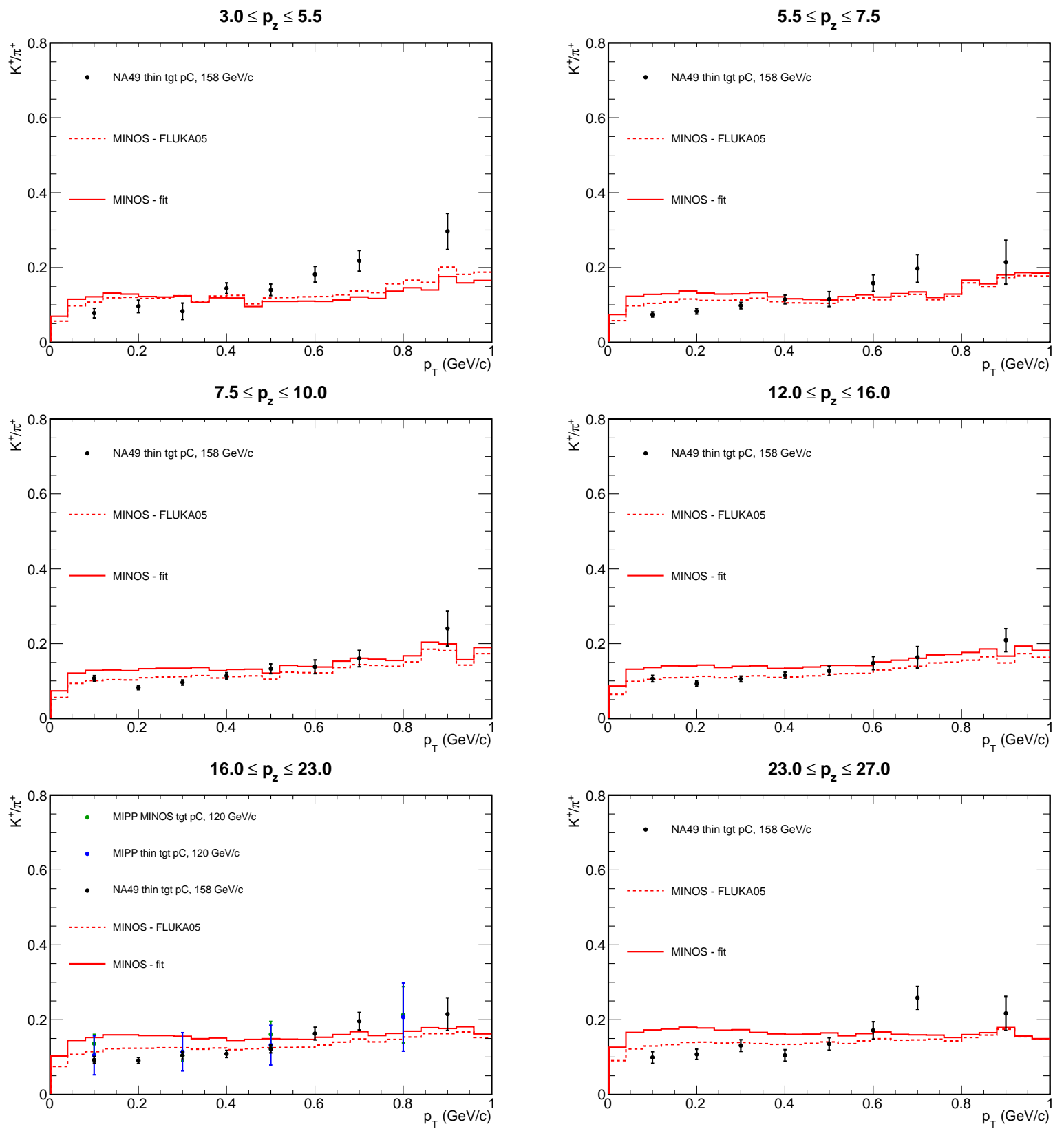

Figure 7.19: Comparison of $\mathrm{K}^{+} / \pi^{+}$as a function of $p_{T}$ for different $p_{z}$ ranges. The solid red line represents the MINOS best fit, the dashed red line is FLUKA05 prediction and the black dots represent the NA49 results in this thesis. The MIPP results for the thin target are in blue and the ones for the MINOS target in green. The $p_{z}$ ranges are expressed in $\mathrm{GeV} / c$. 

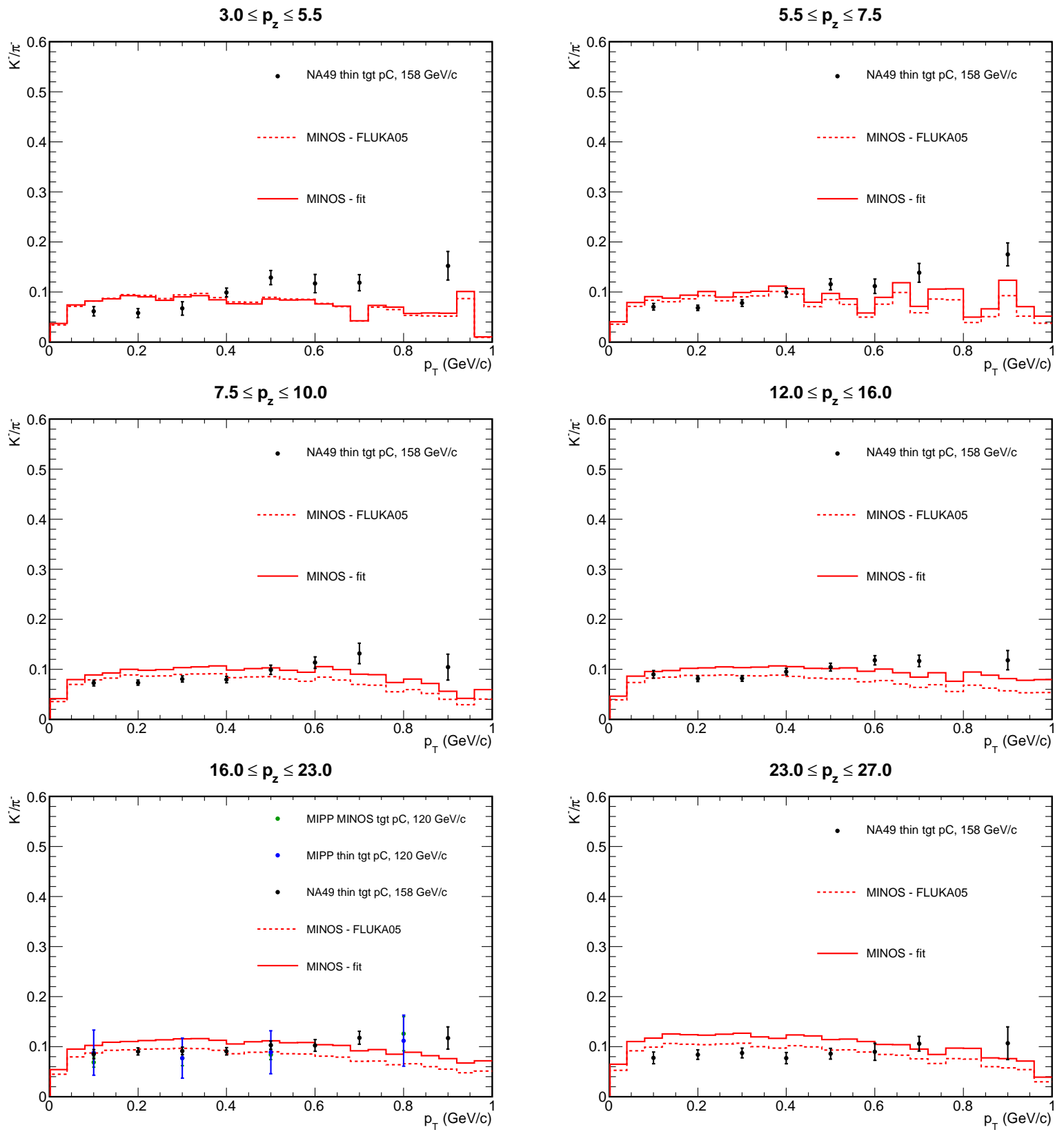

Figure 7.20: Comparison of $\mathrm{K}^{-} / \pi^{-}$as a function of $p_{T}$ for different $p_{z}$ ranges. The solid red line represents the MINOS best fit, the dashed red line is FLUKA05 prediction and the black dots represent the NA49 results in this thesis. The MIPP results for the thin target are in blue and the ones for the MINOS target in green. The $p_{z}$ ranges are expressed in $\mathrm{GeV} / c$. 
simulation. 


\section{Chapter 8}

\section{Sterile neutrino oscillations in}

\section{MINOS}

\subsection{Analysis strategy}

This chapter presents the analysis of sterile neutrino oscillation in MINOS for the case of a third mass splitting of order $\mathcal{O}\left(1 \mathrm{eV}^{2}\right)$. The data analysed corresponds to $7 \times 10^{20}$ protons on target and represent the whole data collected by the MINOS Far Detector between May 2005 and June 2009. The analysis has been performed within the MINOS Neutral Current Working Group, which has analysed the same dataset at the same time as this analysis. The official analysis uses an improved charged current selection [141] which marginally improves the results presented in this thesis. MINOS has performed a "blind analysis", meaning that an unknown fraction of the Far Detector data was not available before having decided the whole analysis procedure. The studies pursued in this thesis are mainly dedicated to the Near Detector preselection, the optimisation of the neutral current selection and the studies of the effects of the systematic errors on the parameters. 


\subsubsection{Neutrino events}

As mentioned in chapter 2, neutrinos of flavour $l$ can interact through charged current interactions of the type $\nu_{l}+N \rightarrow l+X$ or neutral current interactions of the type $\nu_{l}+N \rightarrow$ $\nu_{l}+X$. The beam composition, from MC estimates, is $91.8 \% \nu_{\mu}, 6.9 \% \bar{\nu}_{\mu}$ and $1.3 \% \nu_{e}$ $+\bar{\nu}_{e}$. In the case of $\nu_{\mu}$, charged current interactions are quite easily distinguished by the presence of a $\mu^{-}$in addition to the hadronic activity. Figure 8.1 (left) shows an event display of a MC $\nu_{\mu}$ charged current event at the Far Detector. The true interaction vertex and the products of the interactions are drawn superimposed: it is easy to identify the muon track. On the other hand, neutral current interactions are characterised only by the hadronic activity, that may be reconstructed in the form of a short track. Given the MINOS detector characteristics, there is not much shape information on the hadronic shower development that can be exploited to select neutral current events. Alternative methods to the one presented in this thesis have been studied $[142,141,143,144]$ : the most powerful variables to select neutral current events are the ones which try to identify and reject the muon tracks. The inelasticity $y$ is defined as the fraction of the neutrino energy $E_{\nu}$ that is converted into hadronic energy $E_{\text {hadr }}$ :

$$
y=\frac{E_{\mathrm{hadr}}}{E_{\nu}}
$$

The difficulty of identifying neutral current events from charged current events with high inelasticity $y$ makes the neutral current selected spectrum very impure. Figure 8.1 (right) shows an event display for a MC neutral current event at the Far Detector. The true vertex interaction and the products are drawn on top.

$\bar{\nu}_{\mu}$ are the second most important contribution in the beam. In charged current $\bar{\nu}_{\mu}$ interactions, there is a distinctive presence of a $\mu^{+}$in the final products. As the MINOS detectors are magnetised, the direction of the bending of the $\mu^{+} / \mu^{-}$track allows us to distinguish between $\nu_{\mu}$ and $\bar{\nu}_{\mu}$ charged current interactions. Instead, with neutral current interactions, there is no possibility to distinguish among neutrinos/antineutrinos or even 

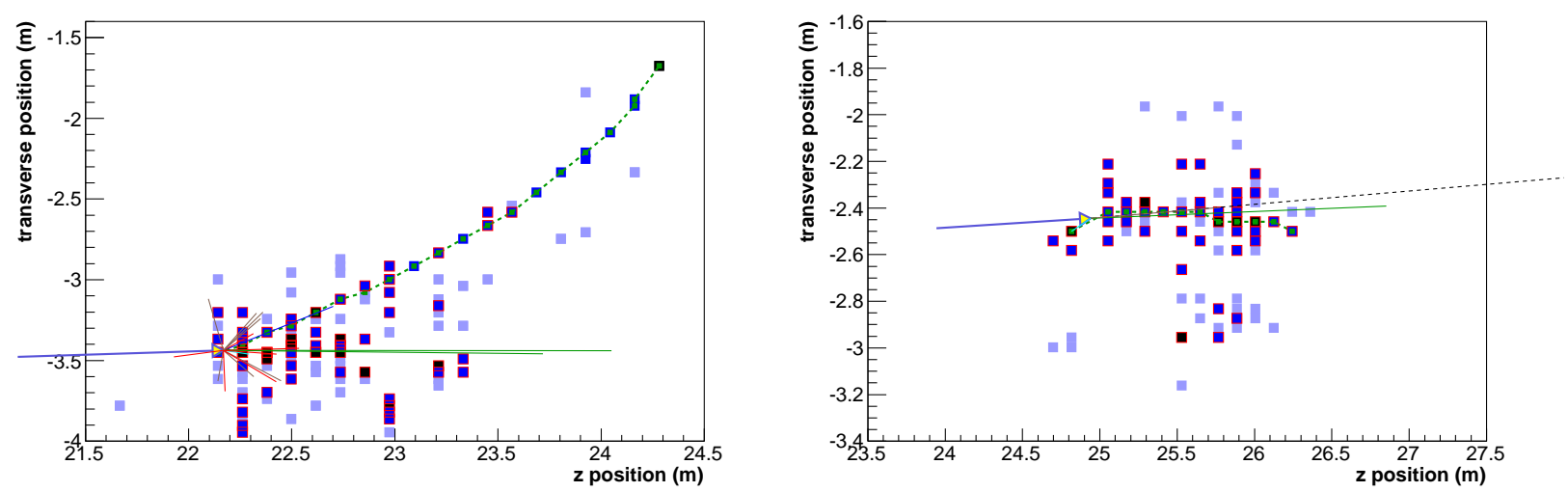

Figure 8.1: The figure shows the event display for a $\nu_{\mu}$ charged current event (left) and a neutral current event (right) for MC events at the Far Detector. The $\nu_{\mu}$ charged current event is characterised by a long muon track, in addition to hadronic activity. The neutral current event is also characterised by the hadronic activity but it also shows some ionisation that has been reconstructed into a short track. Given the characteristics of the MINOS detectors, the selection on neutral current events will be highly impure. The plots are taken from [98].

the flavour.

$\nu_{e}$ and $\bar{\nu}_{e}$ are also produced in the beam. Charged current $\nu_{e}$ events are characterised by the presence of an electromagnetic shower with no possibility to distinguish between a $\nu_{e}$ or a $\bar{\nu}_{e}$. The shower produced by electrons is quite compact: in the longitudinal profile each scintillator+steel plane corresponds to 1.4 radiation lengths and a single strip width corresponds to 1.1 Moliere radii, i.e. the distance which characterises the transverse development of the shower. Figure 8.2 (left) shows a MC charged current $\nu_{e}$ event display at the Far Detector with truth interaction information superimposed. The event display on the right shows the same neutral current event as in figure 8.1 to underline the similarity of the two event topologies at the MINOS detectors. Moreover, neutral current events that produce $\pi^{0}$ in the final state contain electromagnetic showers in the same way as $\nu_{e}$ events. $\nu_{e}$ are a background in the neutral current selected events in MINOS. 

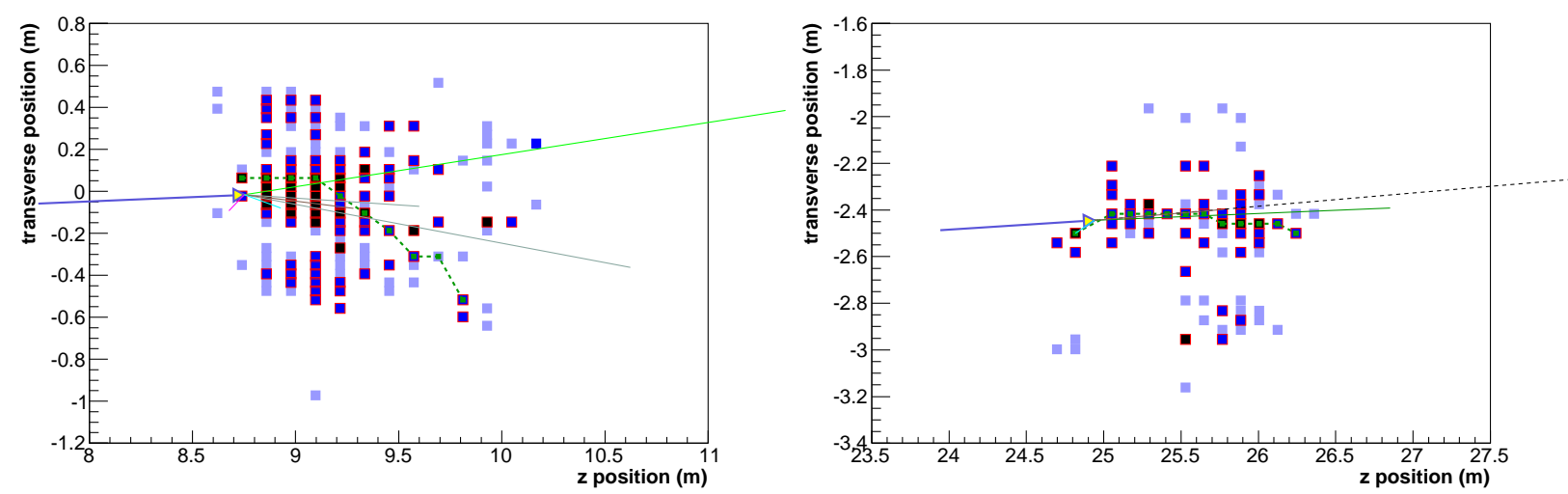

Figure 8.2: The figure shows the event display for a $\nu_{e}$ charged current event (left) and a neutral current event (right) for MC events at the Far Detector. The $\nu_{e}$ charged current event is characterised by a compact electromagnetic shower. The neutral current event is characterised by the hadronic activity that is more transversally spread. Nevertheless, it is immediate to understand that charged current $\nu_{e}$ events will be a background of the neutral current selection. The plots are taken from [98].

\subsubsection{Sterile neutrino oscillations analysis}

The neutral current neutrino energy spectrum is not sensitive to oscillations of neutrinos between active flavours because the neutral current interaction cannot distinguish among flavours. On the other hand, it is possible to study oscillations into sterile neutrinos, which do not couple to the $Z^{0}$ boson, as the signature of this process would be an energy dependent depletion of the neutral current energy spectrum. The formalism used in this analysis is consistent with the theory presented in section 2.3.4. The first step in the analysis is to separate neutral current and charged current interactions and obtain the energy spectra for those events. In the case of $\nu_{\mu}$ charged current interactions, as all the products of the interaction can be detected, the visible energy $E_{\text {vis,cc }}$ corresponds to the energy of the incident neutrino and it is given by the sum of the muon track energy $\left(E_{\text {trk }}\right)$ plus the hadronic shower energy $\left(E_{\text {shw }}\right)$ :

$$
E_{\mathrm{vis}, \mathrm{cc}}=E_{\mathrm{trk}}+E_{\mathrm{shw}} .
$$


The track energy is obtained either from range for contained muons, or from curvature for exiting muons. The resolution on the track momentum has been evaluated to be $5 \%$ from range and $10 \%$ from curvature [80]. The shower energy is evaluated through calorimetry.

Instead, as a neutrino is produced in a neutral current event, the visible energy detected in neutral current events $\left(E_{\text {vis,nc }}\right)$ only corresponds to the hadronic energy of the final $\operatorname{products}\left(E_{\mathrm{shw}}\right)$.

$$
E_{\mathrm{vis}, \mathrm{nc}}=E_{\mathrm{shw}}
$$

From now on, when referring to $E_{\text {vis }}$, it means the definition of visible energy for charged current or neutral current events, according to the selection being used. As a general consideration, it is clear from equation (8.2) that to reconstruct the neutrino energy it is necessary to have a good shower resolution. On the contrary, in neutral current events the resolution on the shower energy has a limited impact as the shower energy in any case carries only a fraction of the original neutrino energy as it is already smeared by the $y$ distribution ( $y$ is defined in equation (8.1)). There is a subtle difference in the definition of the shower energy for charged current or neutral current events. Since frequently the track and shower share hits in charged current events, the hits in common are subtracted from the shower as the corresponding pulse height is already included in the track energy. Instead, in the case of neutral current events, the track is considered only an artifact of the reconstruction and the common hit energy is included in the calorimetric shower energy.

The energy spectra for selected neutral current and selected charged current events are obtained at the Near Detector. The Near Detector data spectra are then used to constrain the prediction of the equivalent Far Detector spectra for selected neutral current and charged current events. The prediction is oscillated according to the oscillation probabilities given in equation (2.44) and then fitted to the Far Detector data to obtain the oscillation parameters. The details of these analysis steps and results are presented in this chapter.

Equation (2.44) expresses the probabilities for a $\nu_{\mu}$ to oscillate into the different neutrino flavors as a function of the mass splitting and angle parameters. With the assumption that 
the sterile neutrino mass splitting is $\mathcal{O}\left(1 \mathrm{eV}^{2}\right)$, the oscillations due to $\left|\Delta m_{43}^{2}\right|=\left|\Delta m_{41}^{2}\right|$ at the Far Detector are rapid and average to 1/2, leaving the probabilities independent of the value of $\left|\Delta m_{43}^{2}\right|$. No sterile neutrino oscillation is expected at the Near Detector baseline if $\left|\Delta m_{43}^{2}\right| \approx \mathcal{O}\left(1 \mathrm{eV}^{2}\right)$. Moreover, as already described in section 2.44, some extra assumptions are required in order to remove extra parameters the analysis is too weakly dependent to determine: $\theta_{14}=0^{\circ}, \delta_{2}=0, \theta_{13}=0^{\circ}$ or $\theta_{13}=12^{\circ}$ and $\delta_{1}=3 \pi / 2$. The parameters left in the probability formula are three angles, $\theta_{23}, \theta_{24}$ and $\theta_{34}$ and one mass splitting parameter $\left|\Delta m_{31}^{2}\right|=\Delta m^{2}$. To first approximation it is possible to think that the $\nu_{\mu}$ charged current spectrum is primarily affected by the survival probability $P\left(\nu_{\mu} \rightarrow \nu_{\mu}\right)$ while the neutral current spectrum is primarily affected by $P\left(\nu_{\mu} \rightarrow \nu_{s}\right)$. This is only a simplification (valid only if there was no selected background); the treatment of the full effects of the oscillation probabilities on the two selected energy spectra is described in details in section 8.6. The oscillation probability $P\left(\nu_{\mu} \rightarrow \nu_{s}\right)$ only mildly depends on the angle $\theta_{23}$, while the probability $P\left(\nu_{\mu} \rightarrow \nu_{\mu}\right)$ has a much stronger dependence. Following the same argument, $\Delta m^{2}$ and $\theta_{24}$ also affect the survival probability of $\nu_{\mu}$ more than the oscillation probability of $\nu_{\mu}$ into sterile neutrinos and could be determined by looking at the depletion of the charged current spectrum. Only the angle $\theta_{34}$ is uniquely determined by looking at a depletion of the neutral current spectrum. For this reason, the analysis exploits both the neutral current and charged current selected spectra by looking at a combined fit of the two spectra. In the whole analysis, a cut on the bending of the $\mu^{-}$track is applied to select only charged current $\nu_{\mu}$ events and reject $\bar{\nu}_{\mu}$.

\subsection{Near Detector preselection}

In a previous analysis [142], it was found that the Near Detector neutral current selected spectrum is affected by an overestimate of events due to the presence of badly reconstructed events which are characterised by a low energy shower. In most of the cases poorly reconstructed events are activity generated in a charged current interaction which 
the reconstruction algorithm fails to recognise as connected to the main activity area and reconstructs as a small independent event. Due to the much higher neutrino flux at the Near Detector, the presence of the badly reconstructed events is unique to the Near Detector and causes Far-Near differences. Near Detector data and Monte Carlo have a large discrepancy that is reduced by introducing a cut-based preselection on two variables chosen for their discriminant power versus poorly reconstructed events.

\subsubsection{Fiducial volume}

Events that deposit part of their energy outside the detector will ruin the overall energy resolution of the sample and need to be removed from the analysis. Moreover, it is also likely that data-MC agreement is worse for those events because the reconstruction algorithm has to rely on only part of the event information. There are two categories of events that have deposition of the energy outside the fiducial volume: non-contained events and leaking events. In non-contained events, the neutrino interaction happens in the detector, but the event also develops outside. For muon tracks it would not be a problem, as the momentum of the muon can be measured from the curvature in the magnetic field. As only calorimetric information is used to reconstruct the shower energy, there is no possibility to recover any pulse height information exiting the detector for showers. Leakage events are due to a wrong assignment of the event vertex resulting in a neutrino interaction which happened outside the detector being reconstructed as fully within the detector.

To assure shower containment and rejection of events leaking from the outside, the event vertex needs to be far enough from the closest edge in the transverse plane. Events which have a vertex less than $50 \mathrm{~cm}$ away from the edge of the detector plane are rejected. This cut removes events which are close to the coil as well. At the same time, it is necessary to reject events which are generated longitudinally outside the detector (reconstructed vertex $z<1.7 \mathrm{~m}$ from the front face of the detector) or which are generated inside the detector, but the distance from the vertex $z$ to the back of the calorimeter is too short to fully contain 

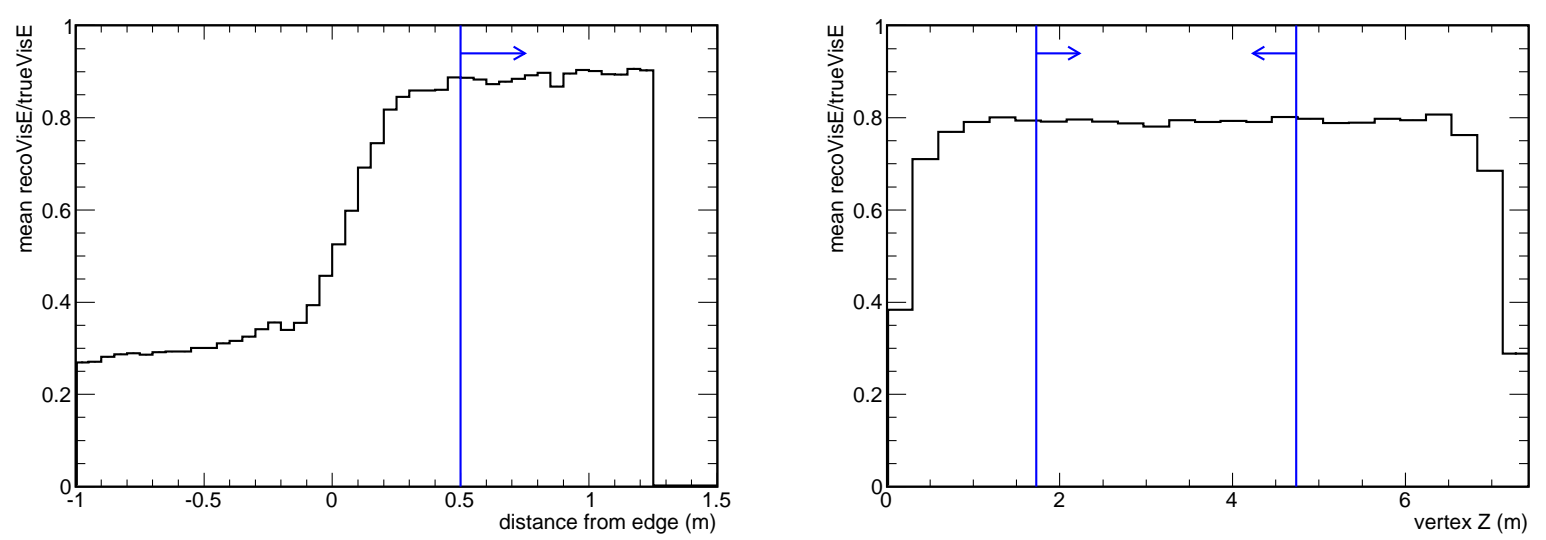

Figure 8.3: The plots show the ratio of reconstructed shower energy divided by true shower energy for $\mathrm{MC}$ events as a function of the distance from the detector edge in the transverse plane (left) and in the longitudinal coordinate $z$ (right). Events having a small value of reconstructed over true shower energy need to be discarded as they would be either non contained events or events leaking from the outside. The blue lines represent the edges of the fiducial volume: the arrows refers to the events kept in the analysis.

showers (reconstructed vertex $z>4.7 \mathrm{~m}$ ).

Figure 8.3 shows the ratio of the reconstructed shower energy to the true shower energy as a function of the distance from the detector edge in the transverse plane (on the left) and from the longitudinal direction (on the right) as obtained from the MC. The fiducial volume cuts are positioned where the ratio is constant and maximal. In the longitudinal direction, it was decided to put the cut more than $1 \mathrm{~m}$ away from the end of the calorimetric part of the detector. Indeed, from MC studies, truly charged current interactions are more likely to be mis-classified as neutral current interactions if closer to the back of the calorimeter because the muon track that enters the spectrometer is not properly reconstructed. The distance from the edge of the detector can be negative for events that have the vertex on the side parts of the fully instrumented planes (described in section 3.3.1), as the variable is evaluated using the distance from the edge of the core region of the detector. Figure 8.4 shows the mean reconstructed shower energy as a function of the distance from the edge (left) and longitudinal position $z$ (right). Data are in black and MC in red. Data are flat in the region of fiducial volume defined and the agreement with the MC is quite good in those regions. 

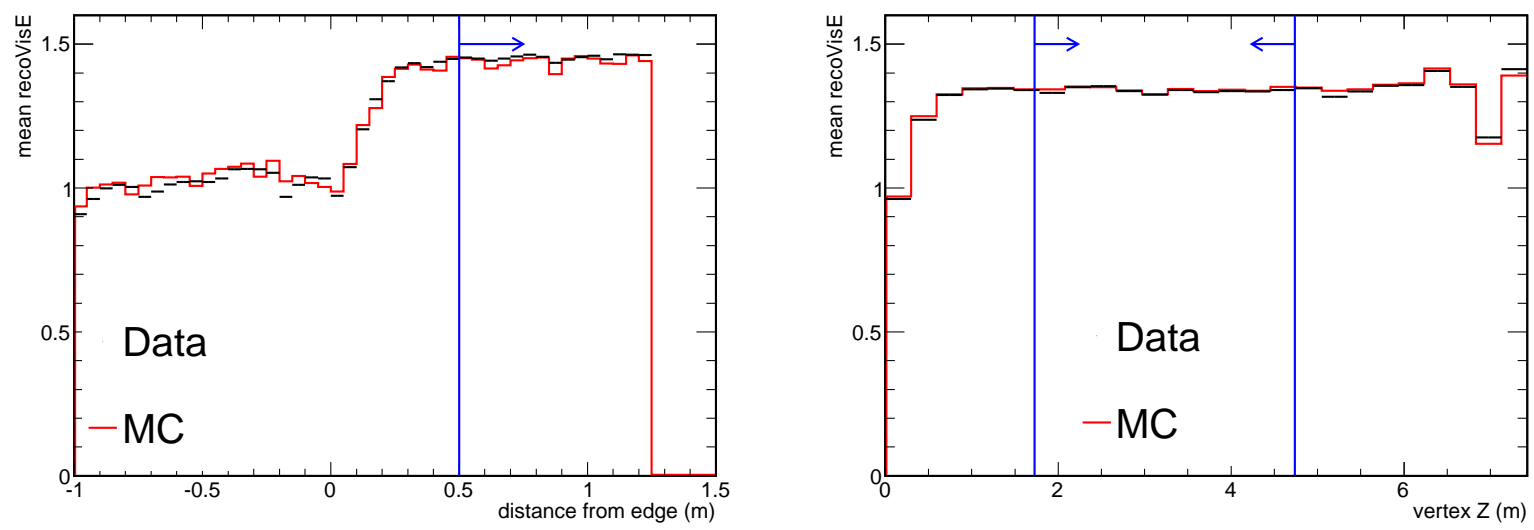

Figure 8.4: The plots show the reconstructed shower energy for data in black and MC in red as a function of the distance from the detector edge in the transverse plane (left) and of the longitudinal coordinate $z$ (right). The mean reconstructed energy in data is also flat as a function of the fiducial volume variables. Moreover, data and MC agree better in the region inside the fiducial volume.

\subsubsection{Definition of background}

The energy spectrum of Near Detector neutral current selected events is affected by badly reconstructed events. In the previous analysis, specific preselection cuts were applied to remove three categories of reconstruction failures [142]: split events, leakage events and incomplete events. Split events occur when a single neutrino interaction results in two reconstructed events. Leakage events have been defined in section 8.2.1 as the events entering from outside the detector. The incomplete event category is a broader classification for the remaining reconstruction failures. In all three categories, the visible neutrino energy may be underestimated and all three are a background for low energy neutral current events. As the Near Detector energy spectrum is used to predict the Far Detector one, reconstruction effects which are present only at the Near Detector need to be removed.

Figure 8.5 shows an example of a split event from the MC. The main event is a charged current event, characterised by the long muon track and some hadronic activity. The arrows show the point where the true neutrino interaction happened. A second area of activity, shown by the hits with red borders, is reconstructed as a separate event. The small secondary event would pass the requirements for being classified as a low energy neutral 


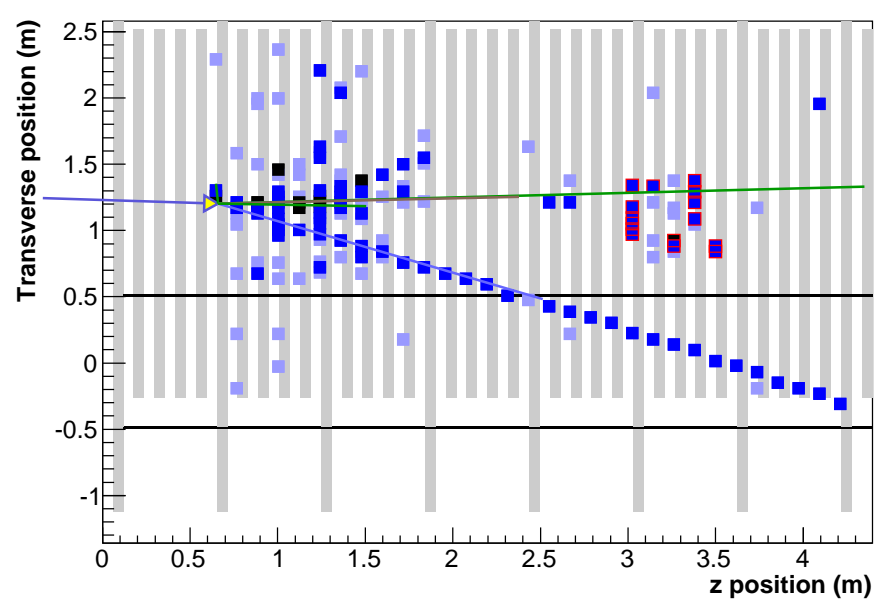

Figure 8.5: An example of a split event. The main event is a charged current event, characterised by the long muon track. The MC true interaction vertex is drawn on top, as well as the true direction of the interaction products. A second event (the red contoured hits) is reconstructed as a small shower only event. The plot is taken from [98].

current event. The preselection cuts described in section 8.2 .3 remove the secondary event. Figure 8.6 shows an example of a leakage event in MC. The true neutrino interaction starts outside of the detector, as indicated by the arrow. Some of the products of the interaction enter the detector and fake a fully contained event as the vertex is erroneously assigned inside the fiducial volume. This event is also cut by the preselection cuts proposed in section 8.2.3.

Regardless of whether they are categorised as split, leakage or incomplete, poorly reconstructed events are characterised in MC by a low value of the ratio of the reconstructed shower energy divided by the true shower energy. The true shower energy is obtained as the sum of the energy of all the non-lepton particles produced in the neutrino event in the MC. The true shower energy does not require hits to be deposited in the detector, thus it describes leakage and non-contained events as well. The definition of the poorly reconstructed background events has been chosen to be:

$$
\frac{\text { reconstructed shower energy }}{\text { true shower energy }}<0.3 \text {. }
$$




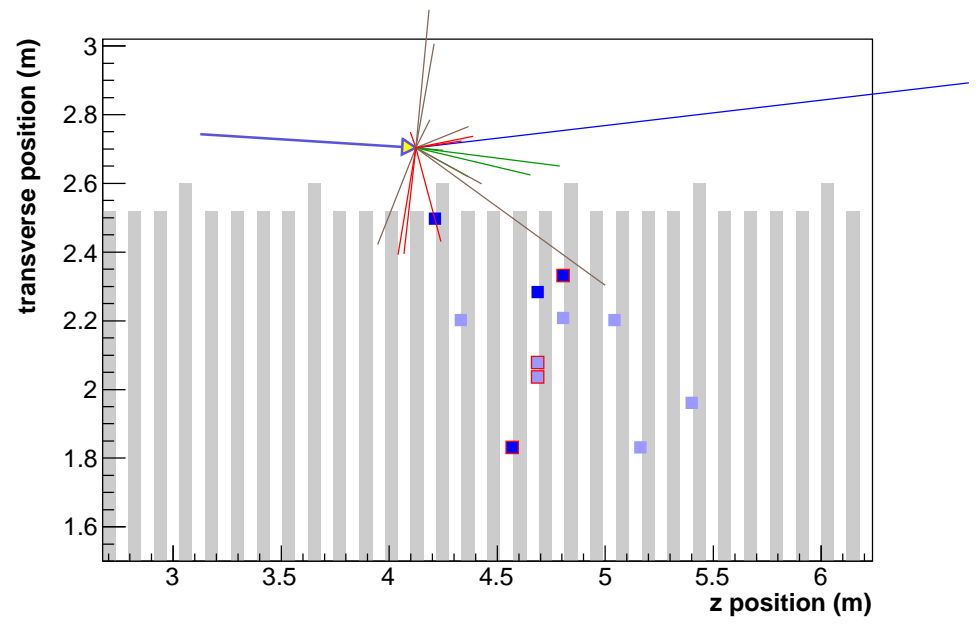

Figure 8.6: An example of leakage event in MC; the true neutrino vertex is drawn on top. The reconstruction algorithm assigns the reconstructed vertex to a high pulse height hit in the middle of the detector causing this events to pass the fiducial volume requirements. There is activity in the detector that is not associated with the event and can be used to veto the event. The plot is taken from [98].

Figure 8.7 shows the distribution of the ratio of the reconstructed shower energy divided by the true shower energy for neutral current selected events. This quantity should in principle be distributed around unity, but a cluster of events at low value $(<0.3)$ is observed. Events that are split or incomplete are shown in green, leakage events are shown in red. Leakage events need to be removed only when the reconstructed shower energy does not describe the energy really deposited in the shower, i.e. when the energy resolution of the shower is compromised. The union of the two topologies of badly reconstructed events is drawn in blue and accounts for all the low values of the reconstructed energy divided by the true shower energy. This is an indication that non-contained events are properly discarded by the fiducial volume cuts described in section 8.2.1.

There is a possible background source that is present in data but is known not to be modelled in the MC: cosmic muons that could cross the detector during a spill trigger. Cosmic muons that pass the neutral current selection would in general be reconstructed as a very steep shower, with a high density of strips in a few planes crossed [142]. A check on the rate of cosmic muons has been performed by looking at the rate of events collected 


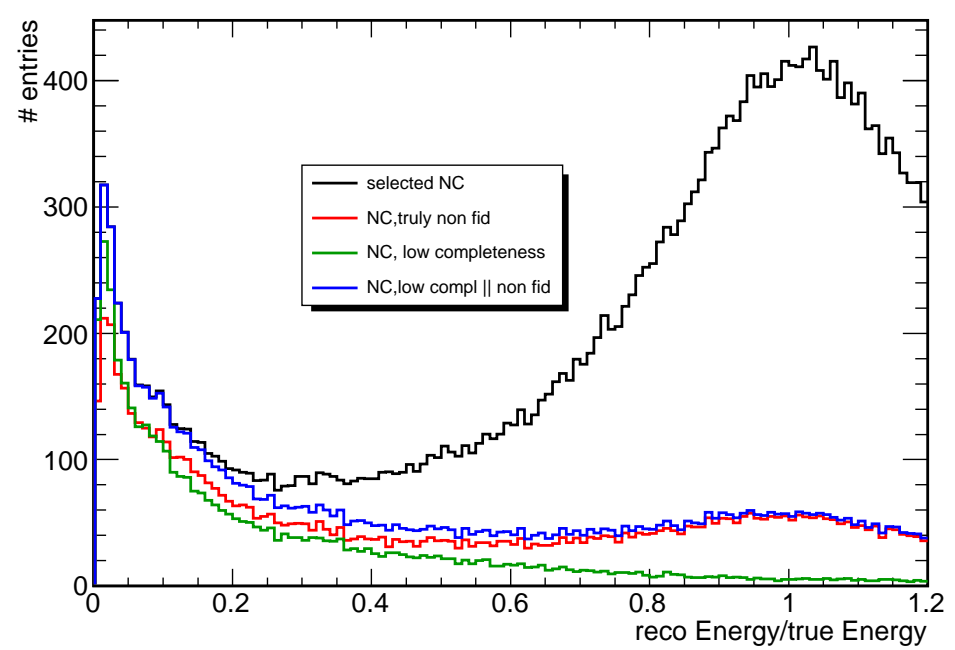

Figure 8.7: The ratio of the reconstructed shower energy divided by the true shower energy for selected neutral current events in black. Two different topologies that contribute to low values of the ratio are shown on top: incomplete events are shown in green and events leaking from outside the detector are shown in red. The union of those two categories account for all the contributions at low values of the variable, as shown by the blue histogram.

during the $1.5 \mu$ s acquisition time window which precedes a spill trigger. Figure 8.8 shows the distribution of the time of the events in respect of the spill trigger time. The plot on the left is for all events, the plot on the right is after applying the fiducial volume cuts. The rate of non beam related accidental events in the $1.5 \mu$ s pre-trigger window is less than $0.2 \%$ of the events occourring in the remaining part of the spill trigger. After applying the fiducial volume cut, no significant fraction of events passes the selection in the first $1.5 \mu$ s. It is safe to neglect the accidental cosmic muons.

\subsubsection{Preselection Variables}

Two preselection variables have been introduced to reduce the poorly reconstructed component. It is expected that when a shower develops longitudinally, it will deposit energy in successive planes. On the other hand, poorly reconstructed events, caused by reconstruction failures have a small number of maximum contiguous planes. Events are cut if the 

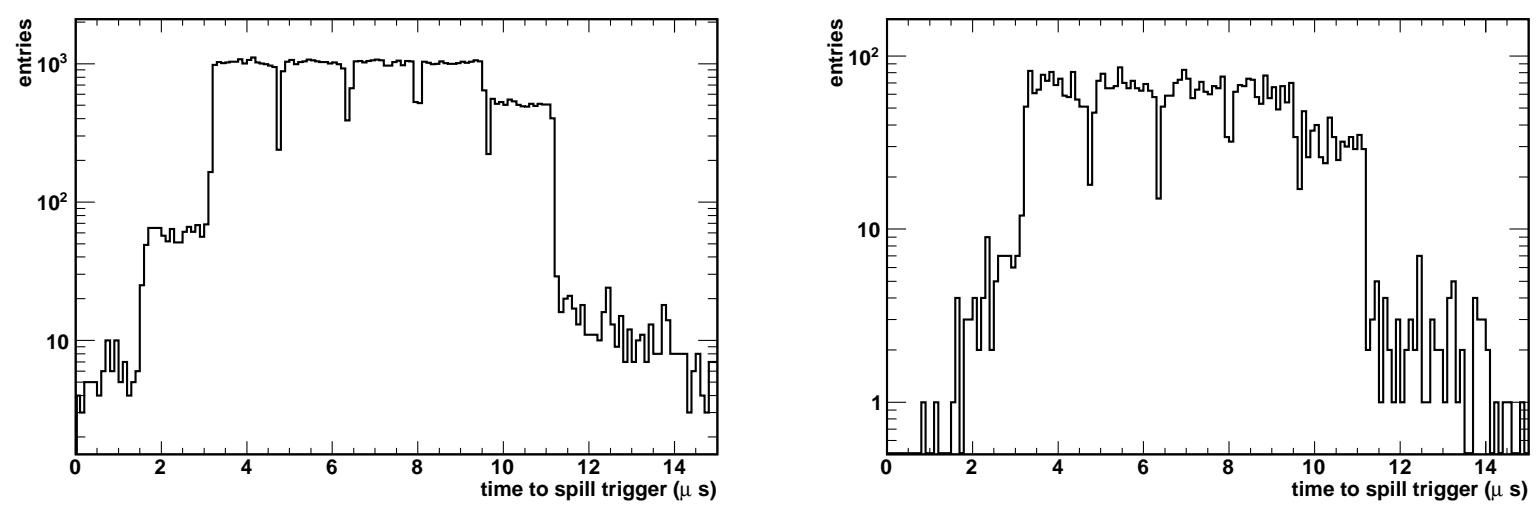

Figure 8.8: The plot shows the distribution of the event time compared to the spill trigger time. On the left is for all events, on the right is after applying the fiducial volume cuts. A spill lasts for about $12 \mu \mathrm{s}$; the shape of the batches in the beam is visible. Only a small fraction of events is detected in the first $1.5 \mu \mathrm{s}$ : the rate of accidental coincidences with the spill trigger is negligible.

variable maximum Consecutive Planes:

maximum Consecutive Planes $<3$.

The upper plot in figure 8.9 shows the distribution of the variable for data, for all MC and for the poorly reconstructed component. The bins in which the contribution of the poor reconstruction is high show a poor data-MC agreement. This cut is quite aggressive: it eliminates all the events that deposit charge on less than 3 planes and $45 \%$ of the events that have hits on 3 (even non-consecutive) planes only. From event-scanning it is possible to observe that events having a small number of hits are poorly reconstructed events [142].

The second preselection variable is defined as the event pulse height divided by the slice pulse height. The concept of slice, introduced in section 3.5, refers to the activity concentrated in a time/space window. The distribution for this variable for data, MC and the poorly reconstructed events is shown in the lower plot in figure 8.9. Events leaking from the outside of the detector will have some activity at the edge of the detector in the same slice as an event, but most likely the activity will not be associated with the event and can be used as a veto. At the same time, split events and incomplete events will also show some 
extra activity that is not associated with the event. Events are cut if the variable slice $P H$ Fraction:

$$
\text { slice PH Fraction }<0.5 \text {. }
$$

As the poorly reconstructed events are a specific background in the neutral current analysis, their effect on the neutral current energy spectrum is compared after all the final selection cuts have been applied. The neutral current event selection will be described in section 8.5.1. Figure 8.10 shows the neutral current selected energy spectrum before applying the preselection cuts (top) and after applying the preselection cuts (bottom) for data, MC and the poorly reconstructed component. The poorly reconstructed component with $E<1 \mathrm{GeV}$ is reduced from $37.5 \%$ to $11.5 \%$ by applying the preselection cuts. The Data/MC disagreement in the energy spectrum is well covered by the systematic errors (which are treated in section 8.7), although the effects of the discrepancies are well mitigated by the two-detector approach in any case.

Figure 8.11 shows the preselection variables maximum Consecutive Planes (top) and slice PH Fraction (bottom) when the cut on the other variable has been applied. In this manner, it is possible to better observe the cutting power that each single variable has. The maximum Consecutive Planes variable cut alone reduces the poorly reconstructed component from $37.5 \%$ to $17.7 \%$. The slice PH Fraction cut alone reduces the poorly reconstructed component to $28.1 \%$.

If $S$ is the signal before applying any cut, $s$ is the signal after applying the selection cuts and $b$ is the background after applying the selection cuts, it is possible to define the efficiency $\varepsilon$ and purity $p$ of the sample as:

$$
\varepsilon=\frac{s}{S} \quad p=\frac{s}{s+b}
$$

We can calculate the efficiency and purity of the sample after applying the preselection cuts and the neutral current/charged current selection cuts. In the case of the neutral current selection, the background is given by the truly charged current events which are 

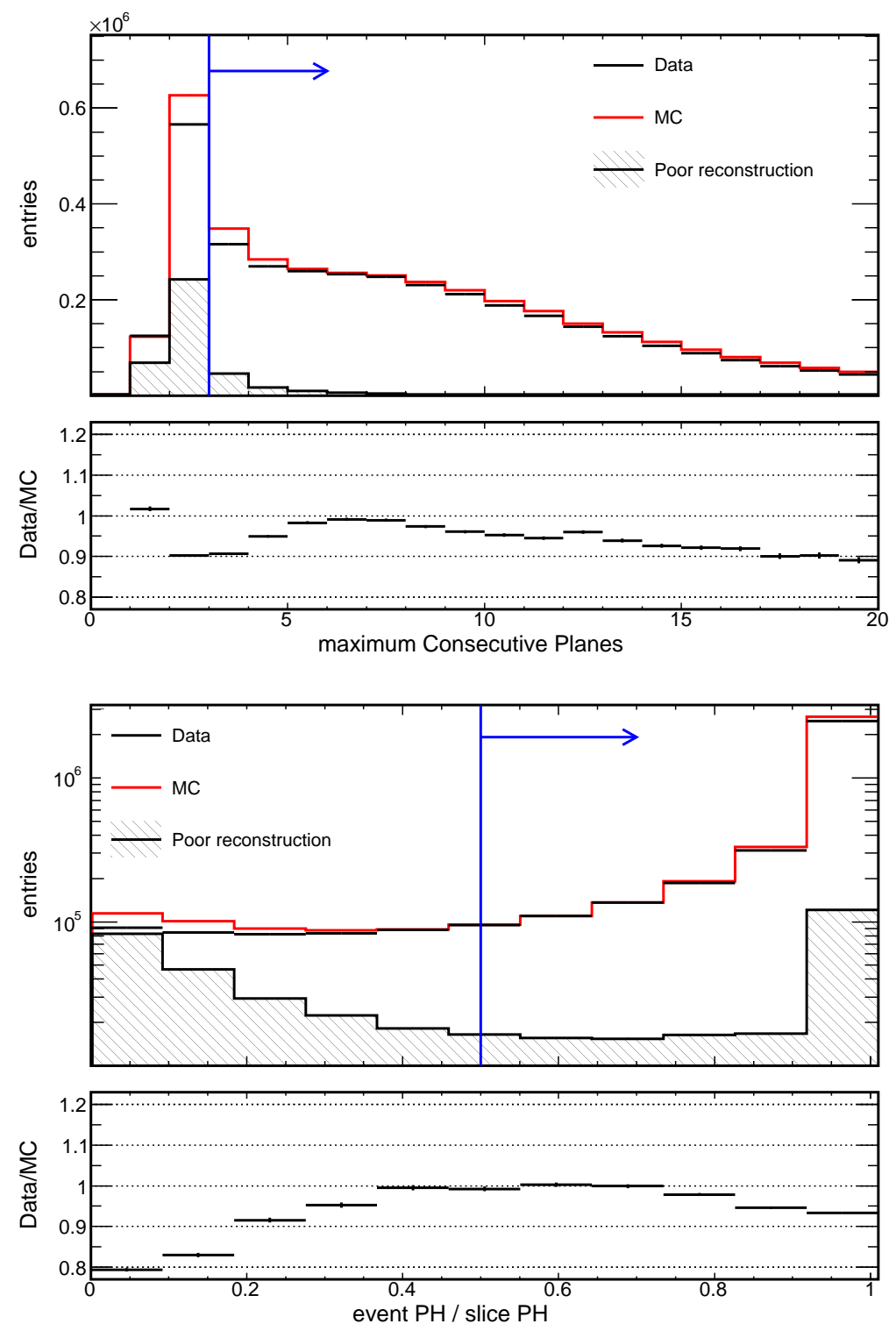

Figure 8.9: The plots show the two preselection variables: the number of maximum consecutive planes in an event is shown at the top. The events pulse height divided by the slice pulse height is shown on the bottom. Data are shown in black, MC in red. The poorly reconstructed component is shown in hatched black. The ratio data/MC is also shown for each variable. The poorly reconstructed component is concentrated in the bins where the data/MC agreement is worse. The blue arrows indicate the events kept in the analysis. 

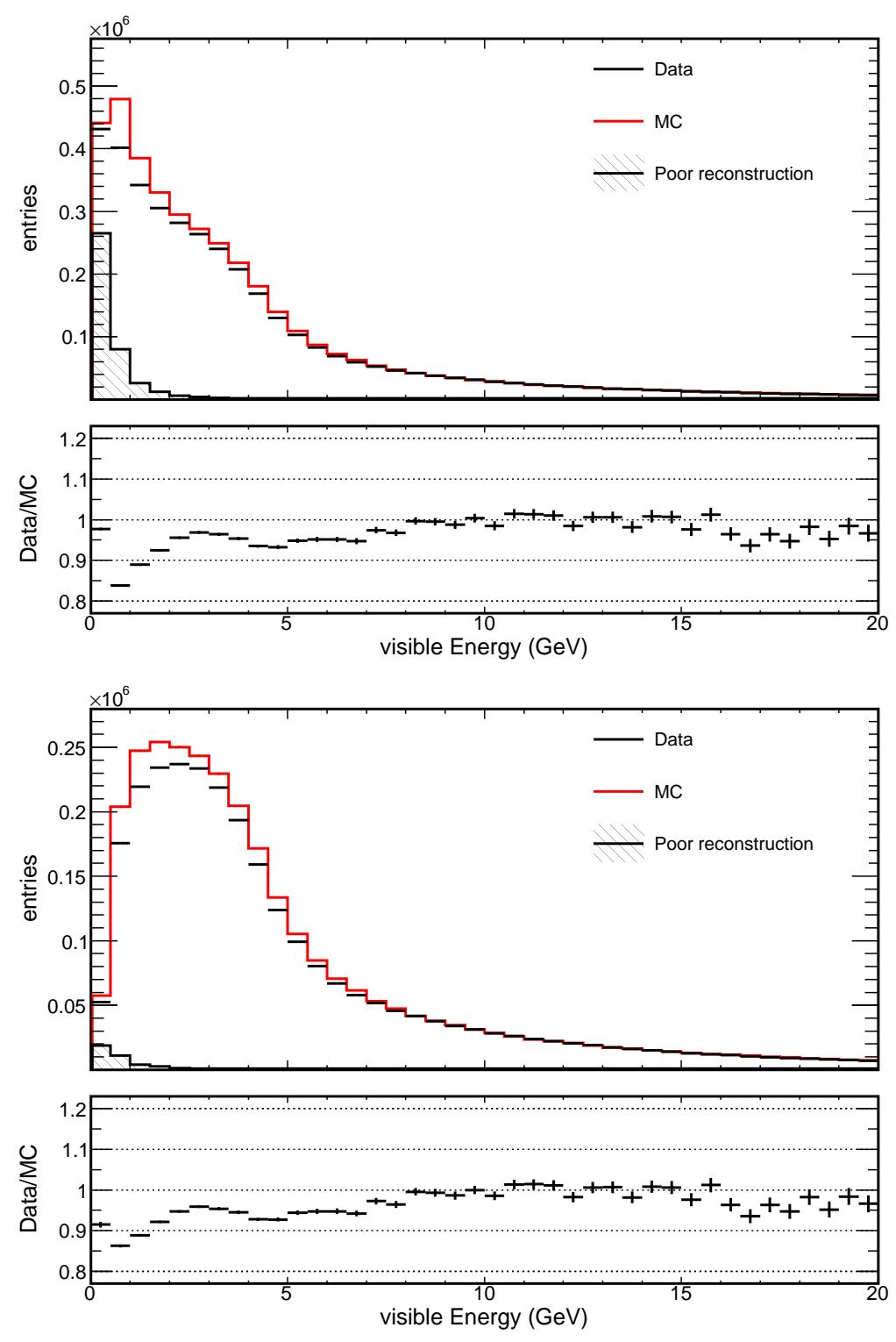

Figure 8.10: The neutral current energy spectra before (at the top) and after preselection cuts (at the bottom). Data are shown in black, MC in red. The poorly reconstructed component is shown in hatched black. The ratio data/MC is also shown. The background percentage with $E<1 \mathrm{GeV}$ is $37.5 \%$ before and $11.5 \%$ after preselection cuts. 

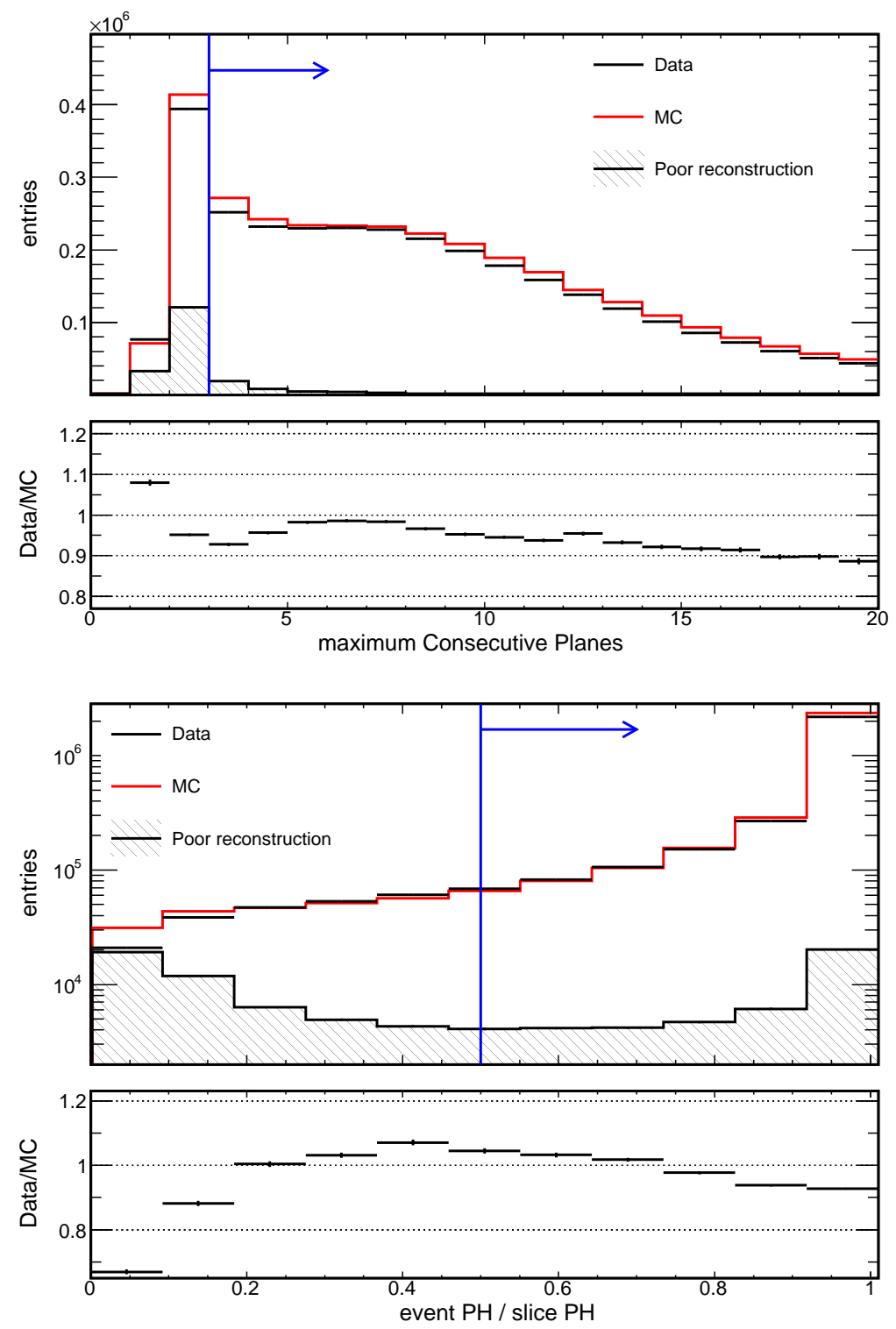

Figure 8.11: The plots show the two preselection variables after applying the cut on the other variable. The number of maximum consecutive planes in an event is shown at the top after requiring the slice PH Fraction cut. The events pulse height divided by the slice pulse height is shown on the bottom after requiring the cut on the maximum Consecutive Planes variable. Data are shown in black, MC in red. The poorly reconstructed component is shown in hatched black. The ratio data/MC is also shown for each variable. The blue arrows indicate the events kept in the analysis. 

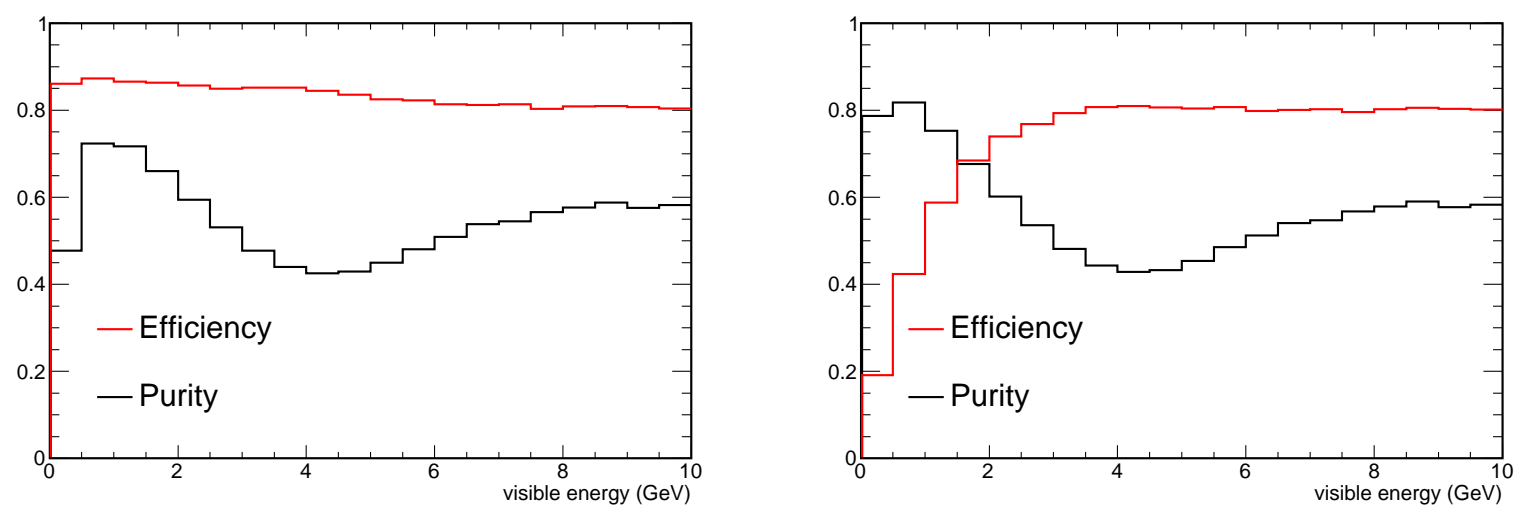

Figure 8.12: Efficiency and purity of selecting neutral current events before (left) and after (right) the preselection cuts. The purity of the sample has been notably increased, at the loss of efficiency.

erroneously selected in the neutral current sample. In the case of the charged current selection, the background is given by truly neutral current events.

Figure 8.12 shows the efficiency and purity of selecting neutral current events (as obtained from MC) before and after applying the preselection cuts. The purity of the neutral current sample has increased as many of the split events are actually originated by truly charged current interactions. The choice of the cut position for the preselection variables has been decided as a compromise between the overall selection efficiency and the minimisation of the systematic errors, which are presented in section 8.2.4.

Figure 8.13 shows the month-by-month stability of the selected neutral current spectrum over the full data taking period after the preselection has been applied. For convenience, Run III has been split into two parts. No significant change in the number of events as a function of the intensity has been seen. To first approximation, in Run I the primary proton beam was run at low intensity while the intensity has particularly increased in Run III. A drop in the number of neutral current selected events is visible over time starting from Run II on. The downwards trend is completely consistent with the drop of the overall number of events and it is due to the graphite target decay. This effect is taken into account in the MC. 

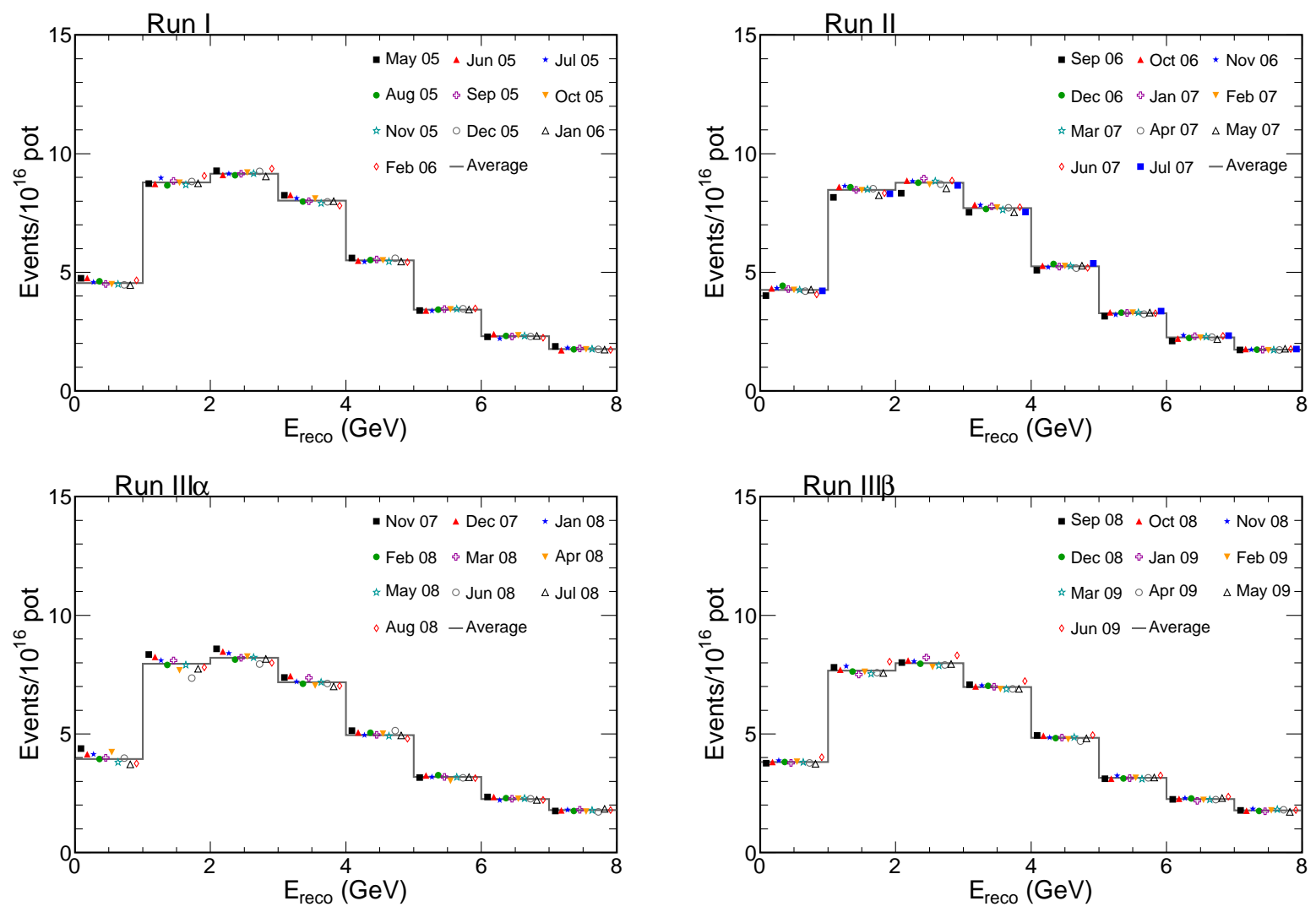

Figure 8.13: Selected neutral current spectra for data, shown month by month (according to the legends) and separated by run number. The solid grey line represents the average spectra in each run period. All months have been normalised to $10^{16}$ protons on target. Indicatively Run I had the lowest intensity of protons on target and Run III had the highest. 


\subsubsection{Systematic error evaluation}

The systematic error associated with the preselection cut has been evaluated as a combination of the uncertainty on the poorly reconstructed component and on the cut position of the preselection variables. Two steps are necessary to fully consider the correlation between the two contributions to the systematic error. The systematic error is given as a function of the visible energy for the neutral current selected spectrum only.

The uncertainty on the poorly reconstructed component has been determined as a scaling factor. For each one of the maximum Consecutive Planes and slice PH Fraction variables, the data entries in each bin $i$ of the histogram are defined as $d_{i}$, the MC entries are given by the number of well reconstructed events $n_{i}$ plus the number of poorly reconstructed events $b_{i}$. A scaling factor $f$ has been determined minimising the $\chi^{2}$ :

$$
\chi^{2}=\sum_{i=1}^{N \text { bins }}\left(\frac{d_{i}-\left(n_{i}+f \cdot b_{i}\right)}{\sigma_{d_{i}}}\right)^{2} .
$$

The overall scaling factor $f$ has been determined as the average of the scaling factor found for the two preselection variables and in 3 ranges of visible energy $(E<0.5 \mathrm{GeV}, 0.5 \leq$ $E<1 \mathrm{GeV}, E \geq 1 \mathrm{GeV})$. The resulting $\pm 1 \sigma$ uncertainty to be applied only on the poorly reconstructed component is: $6 \%$ for $E<0.5 \mathrm{GeV}, 69 \%$ for $0.5 \leq E<1 \mathrm{GeV}$ and $60 \%$ for $E \geq 1 \mathrm{GeV}$. The uncertainties above have been used in the second step of the systematic uncertainty.

After scaling the poorly reconstructed component in the $\mathrm{MC}$, for each cleaning variable, the nominal position of the cut in the MC has been tuned to match the fraction of events rejected by the nominal cut position in the data. As neither of the preselection cuts have any impact on the events with values far away from the cut position, the rejected fraction of events (and not the accepted fraction of events) is matched between data and MC. As the maximum Consecutive Planes is a discrete variable, a weight to match the fraction of events which were cut has been assigned. The procedure was repeated iteratively to match the fractions for both the variables simultaneously. Figure 8.14 shows the distribution of 

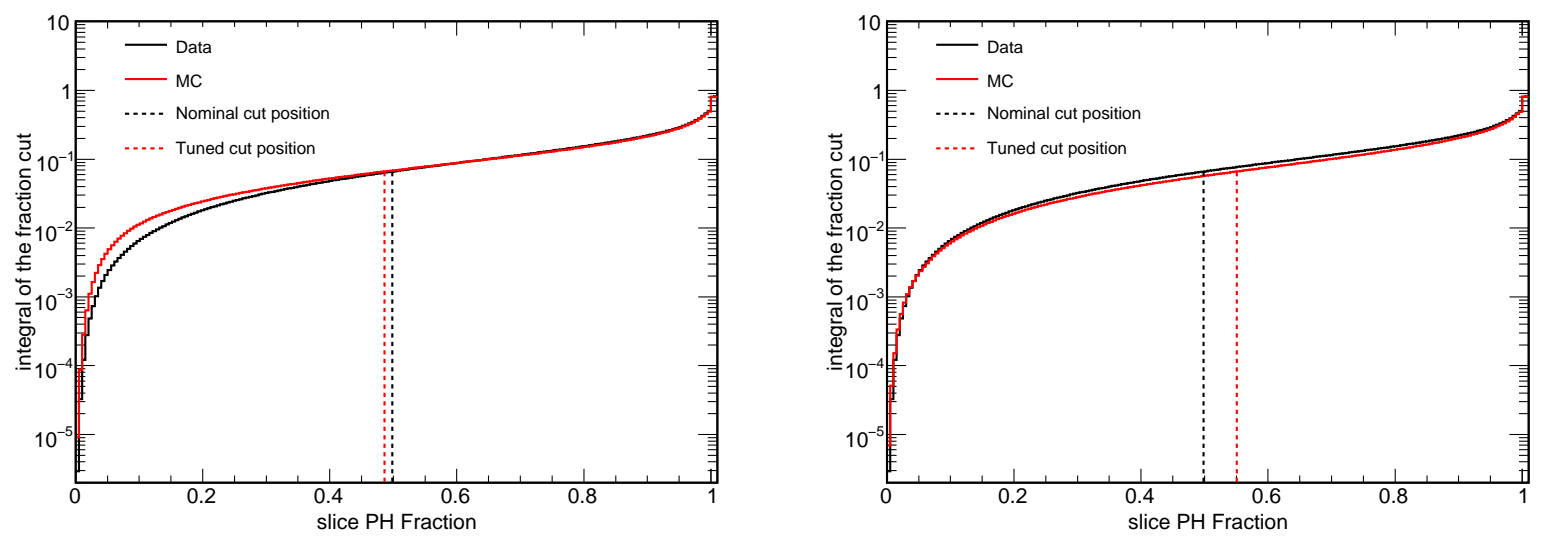

Figure 8.14: The plots show the integral of the fraction of events cut as a function of slice PH Fraction. The plot on the left (right) has been obtained by varying the low completeness component by $+1 \sigma(-1 \sigma)$. The effect of the maximum Consecutive Planes has already been taken into account in this plot. The black histogram represent the integral of the fraction of events cut in data, the red histogram is for MC. The dashed black line indicates the nominal cut position. The red dashed line represents the position of the cut such that the fraction of events cut in $\mathrm{MC}$ is equal to the data one.

the integral of the fraction of events cut as a function of the slice PH Fraction variable. The tuned cut on the maximum Consecutive Planes is already applied at the plots in figure 8.14: the integral of the fraction cut by the slice PH Fraction variable (on the vertical axis) has a maximum at about 0.8 , and not at 1 , as a fraction of events is cut by the maximum Consecutive Planes variable. Black represents data, red MC. The plot on the left has been obtained scaling the poorly reconstructed component by $+1 \sigma$, while the plot on the right has been obtained varying it by $-1 \sigma$. The black dashed line shows the nominal cut position. The red dashed line shows where the cut needs to be positioned in MC so that the integral of the fraction cut (vertical axis) is the same in data and MC.

The systematic error on the energy spectrum has been evaluated from the MC as:

$$
\frac{N_{\text {modified cuts }}(E)}{N_{\text {nominal cuts }}(E)}
$$

where $N_{\text {modified cuts }}(E)$ represents the energy spectrum after the poor reconstruction scaling and the cut position tuning and $N_{\text {nominal cuts }}(E)$ is the nominal MC energy spectrum. The 

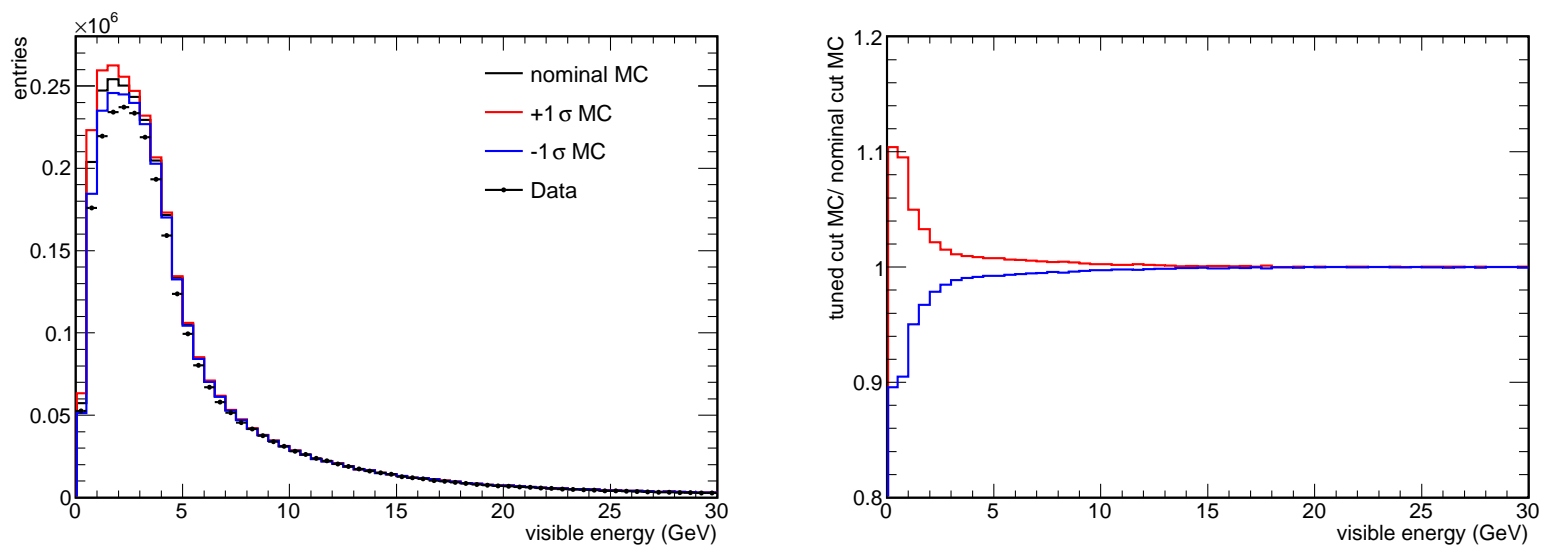

Figure 8.15: The error band for the ND preselection cleaning systematic is shown. The values of the systematic errors assign on the first 5 half $\mathrm{GeV}$ bins are: $10.4 \%, 9.5 \%, 4.8 \%$, $3.3 \%$ and $2.1 \%$. The red line corresponds to the $+1 \sigma$, the blue line to the $-1 \sigma$. The error band is symmetric by constructions.

overall systematic error is the average of the $+1 \sigma$ shift and $-1 \sigma$ shift. An error band, symmetric by construction, is constructed on the energy spectrum, as shown in figure 8.15. The values of the systematic errors assigned on the energy spectrum after all cuts are $10.4 \%$ for $E<0.5 \mathrm{GeV}, 9.5 \%$ for $0.5 \leq E<1 \mathrm{GeV}, 4.8 \%$ for $1 . \leq E<1.5 \mathrm{GeV}, 3.3 \%$ for $1.5 \leq E<2 . \mathrm{GeV}$ and $2.1 \%$ for $2 . \leq E<2.5 \mathrm{GeV}$.

\subsubsection{Discussion on the preselection}

In addition to the two preselection variables used in the final analysis, a different approach to recover split events was studied in earlier days with a fix at reconstruction level. As mentioned earlier, the event builder algorithm associate showers and tracks which are believed to belong to the same neutrino interaction only if they are within the same slice. Given the high rate of neutrinos at the Near Detector, it is not infrequent that the algorithm which divides the activity into slices erroneously splits a real event in two different slices, with no possibility for the later reconstruction algorithms to recover the mistake. This happens $40 \%$ of the times there is a split event. The remaining times, tracks and showers are not assigned correctly even if they are in the same slice. By studying the difference in time between tracks and showers (obtained from the timestamps of the hits), it is possible to 


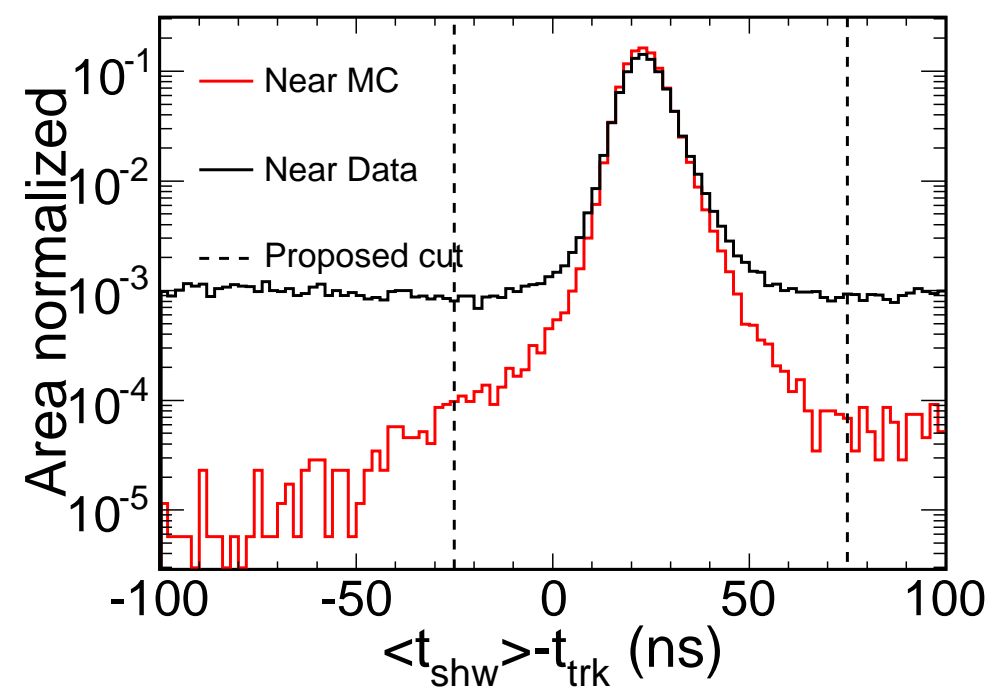

Figure 8.16: The figure shows the distribution of $\left\langle t_{\mathrm{shw}}\right\rangle-t_{\mathrm{trk}}$ in data and $\mathrm{MC}$ in the Near Detector. The peak is due to tracks and showers which belong to the same neutrino interaction, the background is due to random coincidences. The figure is taken from [145].

recover some hadronic activity which has not been correctly associated with the event. This is valid in particular for showers which are slightly displaced from the interaction vertex due to reconstruction failures. This approach was able to recover some of the low energy showers and reattach them to their main event. However, the effect was not enough to remove the overestimate of low energy showers in the neutral current sample. Recently, the Charged Current Working Group, which selects only a charged current spectrum, has adopted a technique very similar to the algorithm studied to recover the full shower energy. Figure 8.16, which is taken from [145], shows the difference in time between the average shower time and the track vertex time for showers and tracks which have their vertices at maximum $1 \mathrm{~m}$ apart. The peak represents tracks and showers which are from the same neutrino interaction, the flat background is given by random coincidences. Even though this approach is successful to recover low energy showers, it is possible to see that the Data and $\mathrm{MC}$ do not agree well in the distribution, mainly due to bad modelling of low pulse height hits on showers.

A better purity in the preselection can be obtained by cutting all events which have 
a difference in time between a chosen interval (40 ns in [142]). In this thesis it has been decided to abandon a temporal cut because the time distribution has some disagreement in the present $\mathrm{MC}$ and reconstruction version and a time cut is largely dependent on the primary proton beam intensity.

\subsection{Intensity effects at the Near Detector}

The beam intensity is a continuous variable distributed between $18 \times 10^{12}$ and $33 \times 10^{12}$ protons per spill. In the previous MINOS publications the MC was simulating only a single average intensity $\left(24.2 \times 10^{12}\right.$ protons per spill). Instead, the MC used in this thesis (and in the future MINOS publications) simulates a discrete number of beam intensities. Ten different beam intensities (ranging from $12.4 \times 10^{12}$ to $32.4 \times 10^{12}$ ) are simulated. The proportion of proton on targets simulated at each beam intensity has been chosen to reflect the protons on target collected in real data in broad bins of intensity centred at the finite MC intensities. The normalisation is performed for each run separately, as documented in $[146]$.

Some of the background in the neutral current selected spectrum comes from reconstruction failures (particularly split events or poorly reconstructed events), it is expected that intensity may affect the neutral current spectrum. A MC which simulates the beam intensity more realistically reduces the systematic error associated with intensity effect as, even when the neutral current selection is affected by the different beam intensities, data and $\mathrm{MC}$ will be affected in the same manner. Nevertheless, the preselection (and selection) variables used in the analysis have been chosen to be quite robust to intensity effects.

Studies have been performed to quantify the dependence of the neutral current selected energy spectrum from any residual mismodelling of the beam intensities. Figure 8.17 shows the number of events per spill as a function of the beam intensity in data and MC for Run I. Data are in black and $\mathrm{MC}$ is in red. As during Run I the primary proton beam intensity was low, only 8 MC intensities are generated in Run I. The data, which have a continuous 


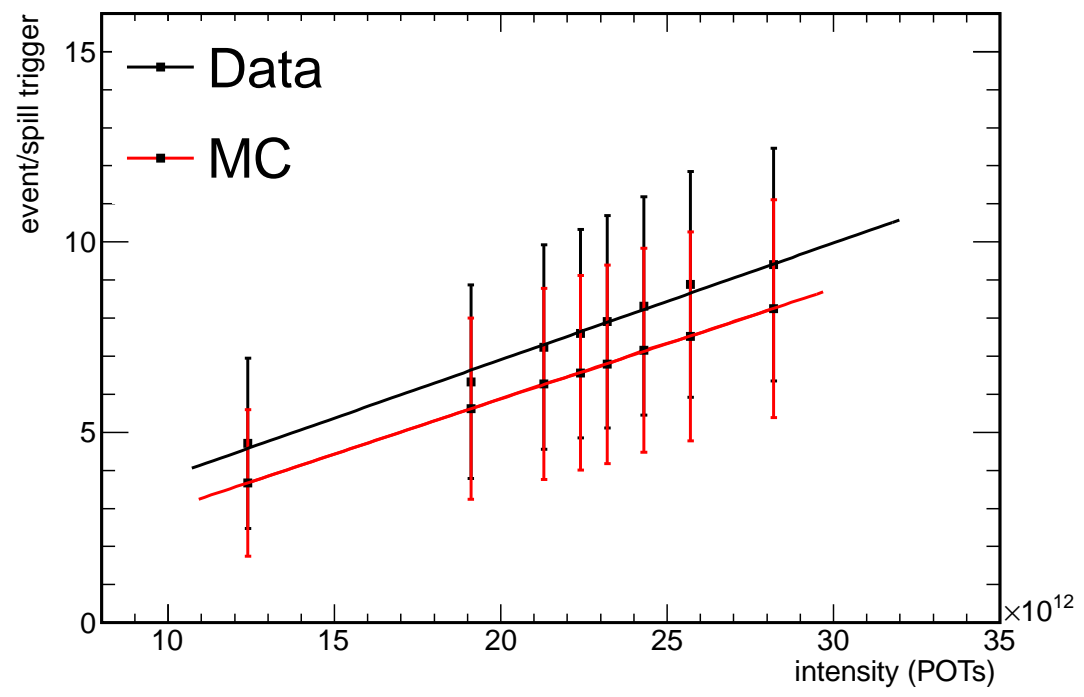

Figure 8.17: The plot shows the number of events per spill trigger as a function of the beam intensity, which is measured in protons on target (PoTs) per spill. Data are in black and the $\mathrm{MC}$ is in red. Both data and MC show a linear trend: a linear fit is superimposed. The intensity in data is a continuous variable while it is discrete in the MC: data have bin binned according to intensities centred around the MC nominal intensities. The error bars represent the RMS of number of events per spill trigger distributions.

distribution of the beam intensity, have been divided into bins having the MC intensities as centres. The trend for both data and $\mathrm{MC}$ is a linear increase of the number of events per spill trigger as a function of the beam intensity. Fitting a straight line, the intercept is consistent with zero in both data and MC. The MC line lies below the data line almost at a constant offset. One can define an "effective" intensity, i.e. the intensity that the MC beam should have to produce the same number of events per spill trigger as the data line. The effective intensity of the MC scales the nominal intensities towards lower values. Assuming the MC effective intensities, it is possible to renormalised the MC to have the same content of protons on target as the data have in the new effective intensity binning scheme. The new normalisation can be used to check that data and $\mathrm{MC}$ agreement improves for intensity dependent variables and evaluate the effect that it has on the energy spectrum.

Figure 8.18 shows the effects of the effective intensity scaling on the preselection variables and on the energy spectrum for Run I. Data are in black, the nominal MC is in blue and the intensity scaled $\mathrm{MC}$ is in red. The plots on the left are (starting from the top): the 
neutral current selected energy spectrum, the maximum Consecutive Planes variable and the slice PH Fraction variable. The plots on the right are the ratio of data to the nominal $\mathrm{MC}$ in blue and the ratio of data to the intensity scaled MC in red. The slice PH Fraction variable is dependent on intensity and the agreement of the MC to the data is improved after the intensity scaling. The maximum Consecutive Planes, which is the leading variable in the preselection, is almost independent of the beam intensity. The energy spectrum is only moderately affected $(<2 \%$ at low energies) by the intensity effects. As the difference induced on the energy spectrum by the residual intensity mismodeling is well below the systematic error quoted for the preselection variables, the remaining intensity effects are considered negligible and no further intensity scaling has been adopted for the analysis.

\subsection{Far Detector preselection}

A set of preselection cuts is applied to the Far Detector. They were originally studied in [21] and have been updated with the current reconstruction version in [147]. Non-contained events and leaking events are removed by the fiducial volume cuts. The non beam related background component was addressed by studying real data, taken out-of-spill, i.e. when some activity in the detector triggers the acquisition, but it is known that it is not a NuMI spill trigger. The sources of non beam related events are cosmic muons, light injection and wavelength-shifting fibre noise.

\subsubsection{Fiducial volume}

The definition of the fiducial volume has been determined with a method similar to the one presented in section 8.2.1 for the Near Detector fiducial volume. In the transverse plane, events were accepted if their vertices are more than $40 \mathrm{~cm}$ distant from the edge of the detector and more than $60 \mathrm{~cm}$ away from the coil. In the longitudinal directions events are accepted if the $z$ component of the vertex coordinate is $0.21 \mathrm{~m}<z<13.72 \mathrm{~m}$ and $16.2 \mathrm{~m}<z<28.96 \mathrm{~m}$. These cuts in the longitudinal direction discard a few planes 

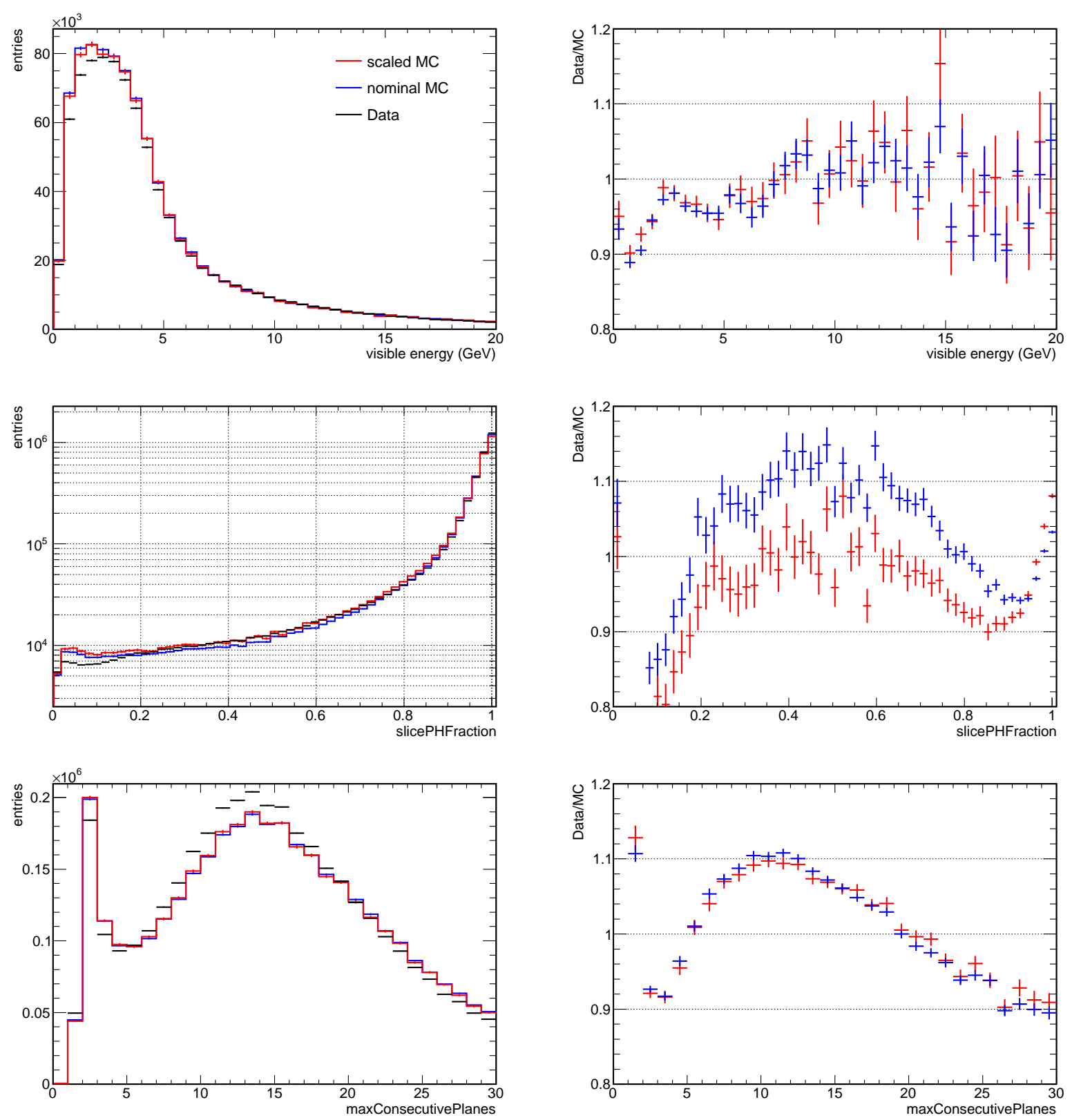

Figure 8.18: The plots on the left show the neutral current selected spectrum for Run I (top), the maximum Consecutive Planes variable (middle) and the slice PH Fraction variable (bottom) for data in black, nominal $\mathrm{MC}$ in blue and the intensity scaled $\mathrm{MC}$ in red. The plots on the right show the ratio of data to the nominal $\mathrm{MC}$ in blue and data divided by the scaled MC in red for the corresponding variables. 

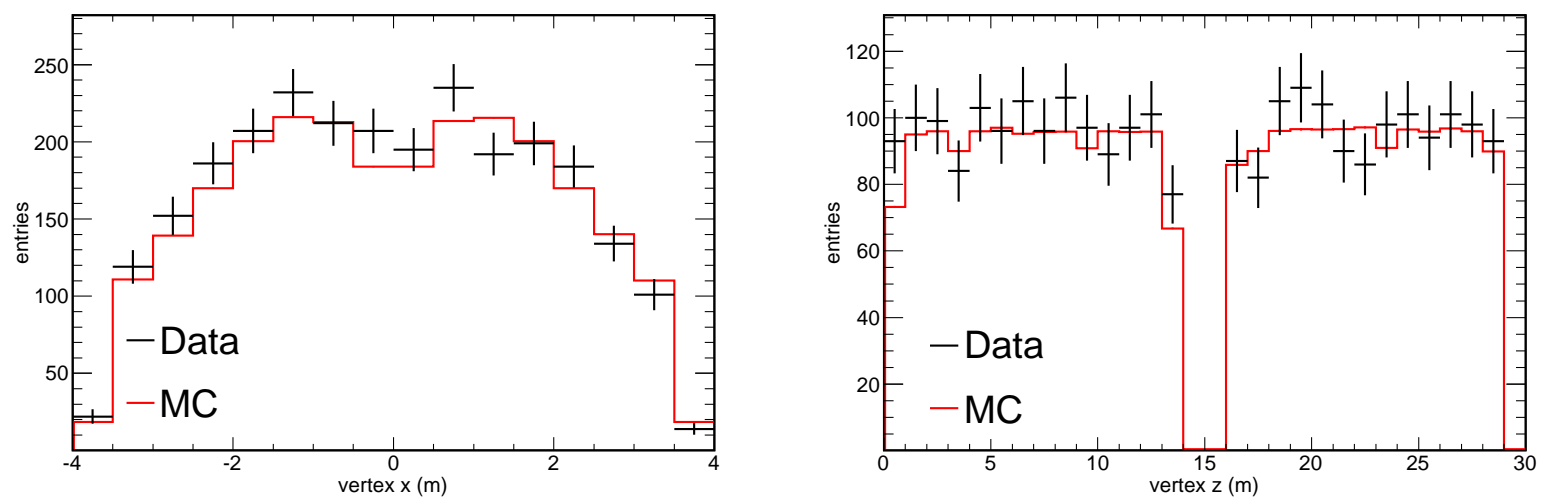

Figure 8.19: Distributions of the neutrino vertices for events that fulfil the fiducial volume requirements in the $x$ coordinate (left) and in the $z$ one (right). Data are in black, the MC prediction is in red. The truly charged current events in the MC have been oscillated in the assumption $P\left(\nu_{\mu} \rightarrow \nu_{\mu}\right)$ according to the oscillation parameters in the previous MINOS publication [57].

at the beginning and end of each supermodule. Figure 8.19 shows the distribution of the neutrino events that pass the fiducial volume cuts at the Far Detector for the $x$ coordinate (left) and the $z$ coordinate (right). Data are shown in black, MC in red. The true charged current events in the MC have been oscillated according to the simple two-flavour oscillation probability introduced in equation (2.24). The parameters for the two-flavour oscillations have been chosen to be $\Delta m^{2}=2.43 \times 10^{-3} \mathrm{eV}^{2}$ and $\sin ^{2}(2 \theta)=1$, according to the charged current analysis results from MINOS [57].

\subsubsection{Light injection}

As the light injection flashes $10^{5}$ times per hour and channels remain dead for up to $30 \mu \mathrm{s}$, light injection events need to be removed. A special photomultiplier is illuminated directly by the LED and is used as a flag that the light injection activity should be excluded. The efficiency of rejection by this method is $99.99 \%$ [147]. A set of three other cuts is used to identify light injection events from their topology. As the light injection is flashed on a single side of the readout, the signal which is transmitted to the other end is attenuated. Instead, for real neutrino interactions, events are expected to happen in a more central location of 
the detector, causing a smaller asymmetry $\mathcal{A}$ between the two read-out ends. Events with $\mathcal{A}>0.55$ are classified as light injection. The energy deposition for light injection events may be greater than for physical events. For high energy deposition, the effect of the nonlinearity of the ADC read-out may become important, causing the asymmetry $\mathcal{A}$ to slightly fall and fail the rejection cuts. For this reason, if the minimum of the energy deposited in one of the two read-out views is just below the range where non-linearity effects are important (pulse height $>1.7 \times 10^{6}$ calibrated ADCs), the other read-out view is considered to be in the non-linearity range and the event is discarded. Light injection events hit lots of planes associated with a single pulser-box. This feature alone is not enough to discard events as physical muon tracks could do the same. The density of planes associated with the second most "active" pulser-box is also considered. For real light injection events, the ratio of the second largest to largest pulser-box density of planes should be small, as only one pulser-box is active at a time. Events are cut if the pulse-box with the highest density of hits has at least $2 \%$ of strips hit and the ratio of the strip hit fraction in the second largest pulser-box to the first one is less than $0.6 \%$. After the dedicated light injection cuts, the inefficiency of rejection improves from $0.01 \%$ to $0.0017 \%$.

\subsubsection{Wavelength-shifting fibre noise}

Studies were carried out to prove that noise is generated by spontaneous light emission from the wavelength-shifting fibres [148]. Events due to fibre noise are characterised by a low number of strips and low pulse height. For real neutrino events, there is a correlation between the number of strips and the pulse height, but this feature is lost for noise events. For this reason a combined cut in the number of strips and the total calibrated pulse height is made. Events are removed if the number of strips is $<9$ and the pulse height $<3750$ calibrated ADCs or if the number of strips is $\geq 9$ and the pulse height $<2000$ calibrated ADCs. 


\subsubsection{Cosmic muons}

The rate of cosmic muons at the Far Detector is $0.5 \mathrm{~Hz}$. As the trigger time window lasts for $100 \mu \mathrm{s}$, the rate of an accidental coincident cosmic muon is expected to be 2 events/day, which is roughly the same as the rate of expected beam neutrinos. For events with a reconstructed track, it is possible to check if its direction is collinear with the beam direction $z$. If the absolute value of the $z$ direction cosine, $\left|p_{Z}\right| / E$ is less than 0.4 , the events are rejected. Very steep cosmic muons will not be reconstructed as tracks, as the reconstruction algorithm is optimised to reconstruct events collinear with $z$, but they would mainly be reconstructed as showers, with a high density of strips in very few planes. If the ratio of strips per plane divided by planes is more than 1 , the event is rejected. Such pathological events will also show a bigger transverse development of the shower: the RMS of the shower is used to reject cosmic muons. As the RMS of the transverse development of the shower increases at higher shower energy, the cut on the RMS is applied as a function of the logarithm of the number shower planes.

\subsubsection{Split events}

Given the rate of events at the Far Detector ( $\approx 2$ measured events/day), the detection of 2 events during the same spill gate is due to either reconstruction failure or non-neutrino background. Split events at the Far Detector are much less frequent than at the Near Detector, but they are removed using the ratio of the event pulse height divided by the whole pulse height collected during the spill. An event is kept if it contains $75 \%$ of the pulse height collected during the spill. As a consequence, only the dominant event passes the selection and the smaller event is rejected.

\subsubsection{Timing requirements}

To ensure that the interaction is due to beam neutrinos, a timing cut around the Global Position System time stamp of the event is required to select only events with timestamp 
within $[-2 \mu \mathrm{s}, 12 \mu \mathrm{s}]$ from the spill gate at the Near Detector.

\subsubsection{Systematic error}

The systematic error associated with the Far Detector preselection has been evaluated in [147]. The position of the cuts have been varied according to the level of the MC mismodelling compared to data. The effect on the energy has also been considered. The systematic errors associated with the Far Detector preselection have been evaluated as two separate components: the error associated with the noise removal and with the cosmic cuts. Table 8.1 shows the systematic errors associated with the two distinct sources of errors. Even summing the two contributions in quadrature, the systematic errors associated with the Far Detector preselection are small compared to the Near Detector preselection ones.

\begin{tabular}{|c|c|c|c|c|c|}
\hline & \multicolumn{4}{|c|}{ Energy $(\mathrm{GeV})$} \\
\hline systematic & $<0.5$ & $0.5-1$ & $1-2$ & $2-3$ & $\geq 3$ \\
\hline Noise cuts & $4.9 \%$ & $1.0 \%$ & $0.6 \%$ & $0.4 \%$ & $0.5 \% \cdot e^{-E / 7.1} \oplus 1.6 \% \cdot e^{-E / 1.1}$ \\
\hline $\begin{array}{c}\text { Cosmic ray } \\
\text { cuts }\end{array}$ & $1.1 \%$ & $2.7 \%$ & $2.3 \%$ & $2.1 \%$ & $7.4 \% \cdot e^{-E / 0.98} \oplus 1.2 \% \cdot e^{-E / 5.5}$ \\
\hline
\end{tabular}

Table 8.1: The table shows the systematic error associated with the Far Detector cosmic ray and noise removal cuts as a function of the visible energy $E$ (expressed in GeV). Table taken from [147].

\subsection{Selection of neutral current and charged current events}

Neutral current and charged current event samples are selected in both data and MC. As the MINOS detectors are coarse calorimeters, long muon track will be well identified, but some ambiguity in identifying tracks will remain for short muon tracks. Neutral current events can also have a track, which is typically given by the ionisation of a pion or proton. Charged current events that have a high inelasticity $y$ have hadronic activity which prevails on the short muon track and are difficult to distinguish from neutral current events. 
It has been chosen to select neutral current events first, the charged current selection is then applied to the events that do not satisfy the neutral current requirements. Using a dedicated charged current selection, rather than using all events failing the neutral current selection, improves the sensitivity of the charged current spectrum [149]. On the other hand, no gain has been found in applying the charged current event selection first and then the neutral current one on the failing events.

\subsubsection{Neutral current selection}

The neutral current selection uses a two variable cut-based approach. The first cut to be applied is on the number of planes in the event, evaluated as the difference between the last active plane and the first active plane in the event. Long muon tracks (low y charged current events) will extend for many planes: if an event extends for 47 planes or more, it is rejected by the neutral current selection. The second variable, called the "track extension", is defined as the extension in planes of the shower minus the extension in planes of the track. Charged current events will cluster at negative values of this variable, as the track will typically protrude more than the shower. Events pass the neutral current selection if the track extension is greater than -6. Note that if the event has no track, the track extension variable will be positive and the event will be selected as neutral current.

The position of the cuts has been chosen using the Far Detector MC and by scanning the values of the variables for which the figure of merit $\varepsilon \times p /(2-p)$ is maximal. The choice of this particular figure of merit is due to the fact that it minimises the statistical error on the selected events [150]. Figure 8.20 shows the surface of the figure of merit as a function of the two selection variables (on the left) and the efficiency and purity of the selected sample as a function of the visible energy (on the right).

Figure 8.21 shows the selection variables at the Far Detector: the event length in planes (left) and the shower minus the track length in planes (right). Data are in black, MC is in red

and the charged current background is in hatched black. At the Far Detector, real charged 

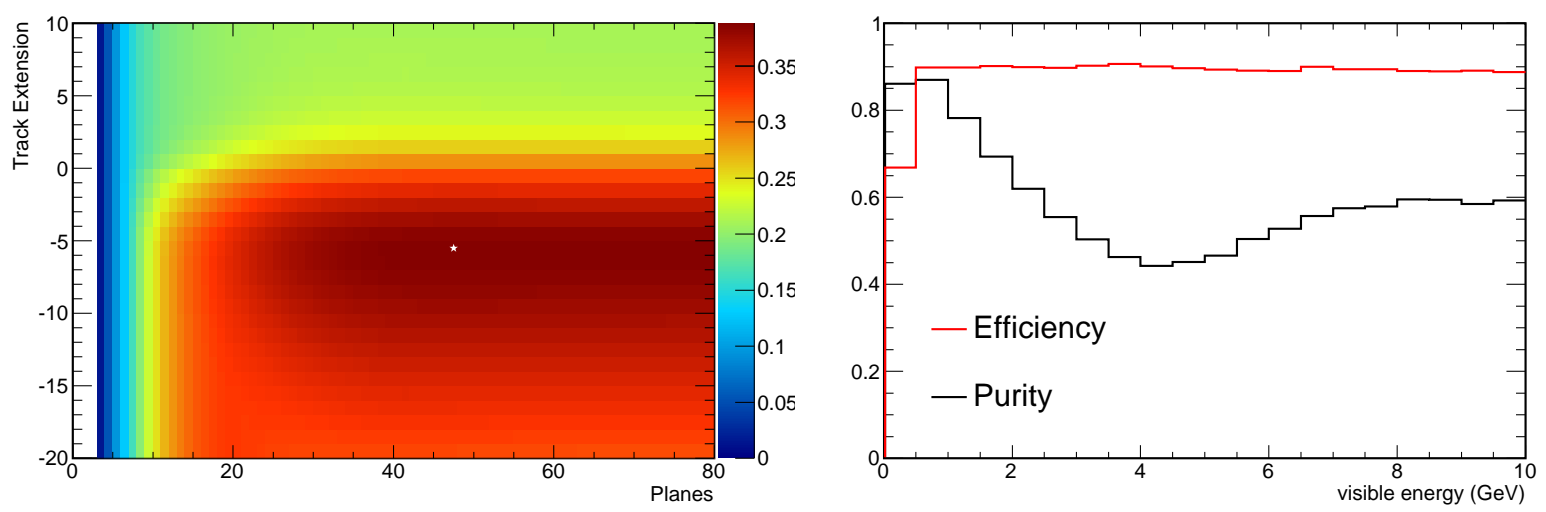

Figure 8.20: The plot on the left shows the figure of merit $\varepsilon \times p /(2-p)$ surface as a function of the track extension and number of planes variables. The star marker indicates the maximum value of the figure of merit. The plot on the right shows the efficiency and purity of the selected neutral current sample as a function of the visible energy. Both plots have been obtained using the unoscillated Far Detector MC.

current $\mathrm{MC}$ events are weighted for $\nu_{\mu}$ to $\nu_{\tau}$ oscillations, according to equation (2.24) and the parameters in [57]. Figure 8.22 shows the selection of neutral current variables at the Near Detector: the event length in planes (left) and the shower minus the track length in planes (right) with the same colour scheme as in figure 8.21. The selection has been tuned on the Far Detector MC, but the same cuts are applied to the Near Detector to minimise the impact of the selection variable systematic mismodelling in the prediction of the Far Detector spectrum.

\subsubsection{Charged current selection}

The charged current selection was developed in [80] and uses three probability density functions $f_{i}\left(x_{i}\right)$ which are constructed on the following variables:

- The number of planes; as already mention charged current events which have a muon track are generally longer than neutral current events.

- The fraction of the total signal of the event in the reconstructed track, which is related to the $y$ distribution of the event. Real neutral current events will have a small fraction of the energy in the track, which is only an artifact of reconstruction. 

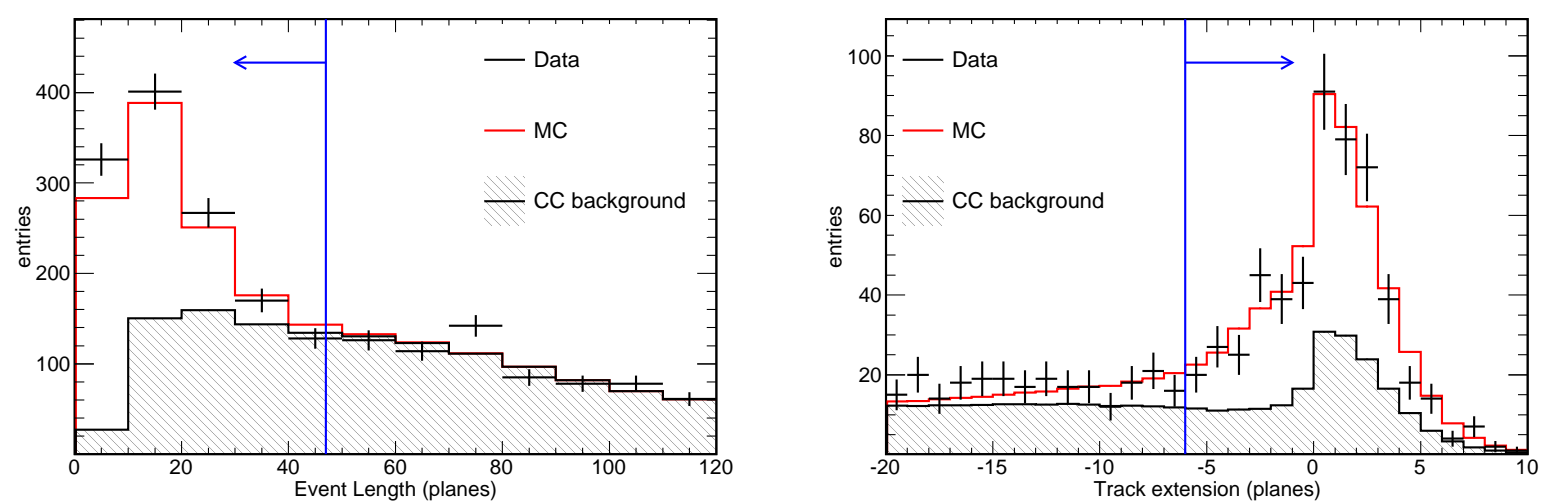

Figure 8.21: The neutral current selection variables at the Far Detector: the event length in planes on the left and the track extension on the right. The effect of the oscillation probability $P\left(\nu_{\mu} \rightarrow \nu_{\mu}\right)$ have been accounted for in the MC according to the two-flavour model. Far Detector data are in black, the oscillated MC is in red and the charged current background (from MC) is indicated by the hatched black area.
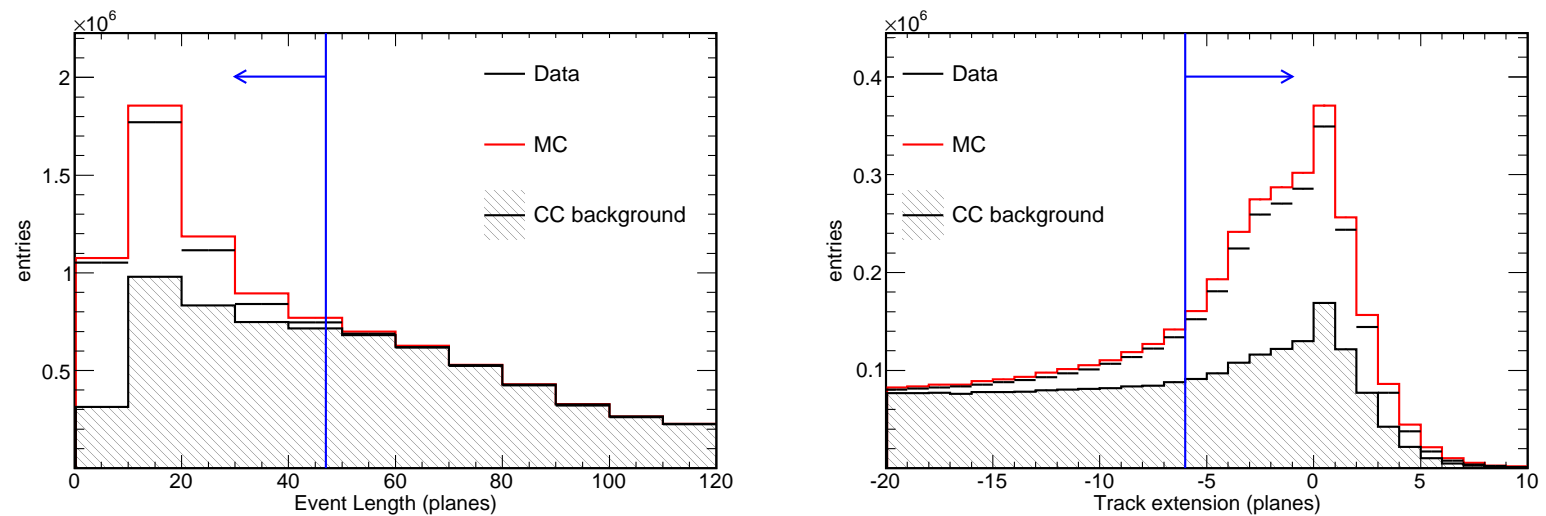

Figure 8.22: The neutral current selection variables at the Near Detector: the event length in planes on the left and the track extension in planes on the right. Near Detector data are in black, the MC is in red and the charged current background (from MC) is indicated by the hatched black area. 

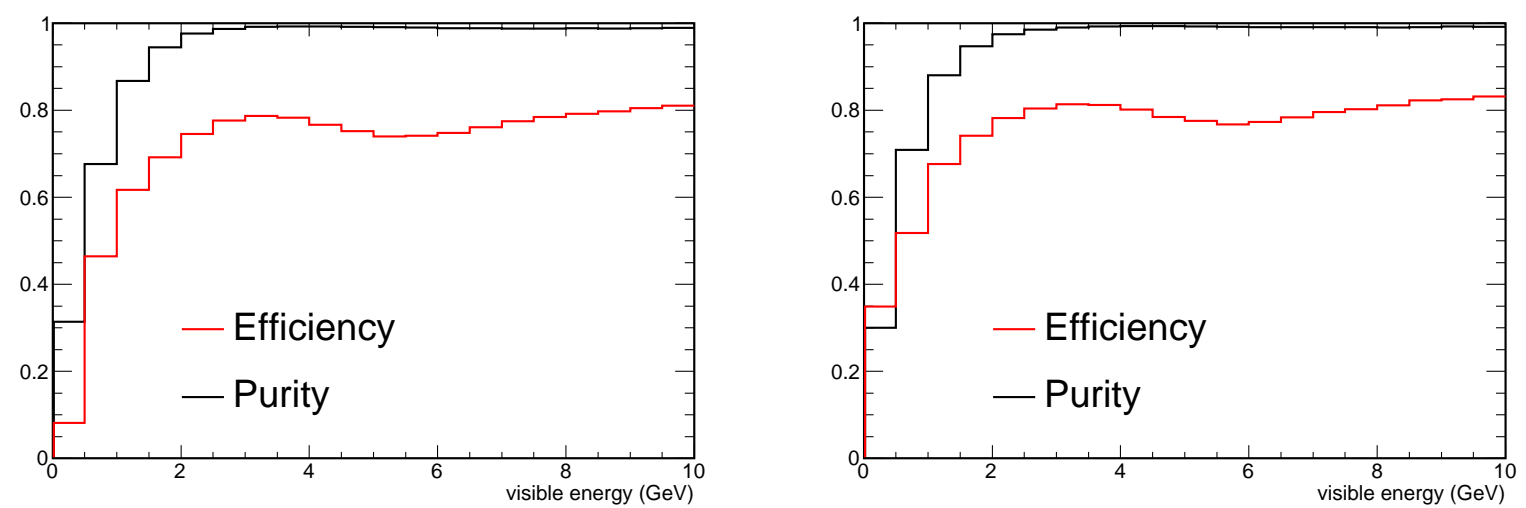

Figure 8.23: The plots show the efficiency and purity of selecting charged current events at the Near Detector (left) and at the Far Detector (right).

- Track signal per plane, which is related to the energy deposition of minimum ionising particles. Real muon tracks typically ionise only a hit per plane in the detector.

The probability of an event to be either charged current $\left(P_{\mathrm{CC}}\right)$ or neutral current $\left(P_{\mathrm{NC}}\right)$ is obtained by multiplying the probability density functions for the three variables used:

$$
P_{\mathrm{CC} / \mathrm{NC}}=\prod_{i=1}^{3} f_{i}\left(x_{i}\right)_{\mathrm{CC} / \mathrm{NC}} .
$$

An event selection parameter has been defined [80] as:

$$
S=-\left(\sqrt{\ln \left(P_{\mathrm{CC}}\right)}-\sqrt{-\ln \left(P_{\mathrm{NC}}\right)}\right)
$$

Events are selected as charged current if $S>-0.2$ at the Far Detector and $S>-0.1$ at the Near Detector. The different cut has been chosen to increase the purity of the Near Detector and make it more similar to the Far Detector one.

Figure 8.23 shows the efficiency and purity of selecting charged current events at the Near Detector (left) and at the Far Detector (right). 


\subsection{Far Detector prediction}

The Near Detector data are used to constrain the MC and give a more robust prediction of the Far Detector energy spectrum. In the $i$-th bin of reconstructed visible energy, the Far Detector (FD) predicted spectrum is obtained from the Far Detector oscillated MC spectrum corrected for the ratio of the Near Detector (ND) content in data divided by the Near Detector content in $\mathrm{MC}$ in the same energy bin:

$$
\mathrm{FD}_{i}^{\text {prediction }}=\frac{\mathrm{ND}_{i}^{\text {data }}}{\mathrm{ND}_{i}^{\mathrm{MC}}} \mathrm{FD}_{i}^{\mathrm{MC}}
$$

The method is often referred to as the "Far/Near" method as it can be interpreted as the Far/Near correction from the MC that needs to be applied to the Near Detector data to predict the Far Detector spectrum. Equation (8.9) is used to predict the spectrum of both neutral current selected and charged current selected events separately.

The prediction of the Far Detector spectrum based on the Near Detector one is technically referred to as "extrapolation". The extrapolation from the Near Detector to the Far Detector in the fashion shown by equation (8.9) is successful as the two detectors are identical to first approximation. Moreover, it is required that the angles subtended by the beam at the two detectors and that the efficiencies and purities of selecting events are similar at the two detectors.

Figure 8.10 (bottom plot) shows the data/MC ratio for selected neutral current events at the Near Detector. The binning used in figure 8.10 is $0.5 \mathrm{GeV} /$ bin, while at analysis level $1 \mathrm{GeV}$ bins are used in the Far/Near extrapolation correction. Nevertheless, it is possible to see that the high energy tail of the neutral current spectrum is well described by the beam reweighted MC. Still some discrepancies are present at lower shower energy (low neutrino energy and low $y$ events), where the MC is not able to fully describe the data and the correction become more important.

The value of $\mathrm{FD}_{i}^{\mathrm{MC}}$ for the Far Detector $\mathrm{MC}$ in equation (8.9) is obtained from the 
MC after applying a weight corresponding to the oscillation probability as a function of true neutrino energy in $0.1 \mathrm{GeV} /$ bin [98]. The conversion from the oscillated spectrum as a function of true neutrino energy to reconstructed neutrino energy is performed using a MCbuilt conversion matrix that relates the $j$-th true energy bin to the $i$-th bin in reconstructed energy [98].

In the prediction of the Far Detector selected and oscillated energy spectrum, it is important to take into account possible backgrounds that may oscillate as well. The Far Detector charged current predicted spectrum $\nu_{\mu}$ is oscillated according to $P\left(\nu_{\mu} \rightarrow \nu_{\mu}\right)$. A background to the charged current selected spectrum is given by misidentified neutral current events. The neutral current events are assumed to only disappear according to $P\left(\nu_{\mu} \rightarrow \nu_{s}\right)$. When $\nu_{\mu}$ oscillate to $\nu_{\tau}$, a small fraction of $\nu_{\tau}$ has a charged current interaction which produces a $\tau$. $\tau$ are short lived and they may decay leptonically into a $\mu$, which would be selected into the $\nu_{\mu}$ charged current spectrum. The efficiency for a charged current $\nu_{\tau}$ to be selected in the $\nu_{\mu}$ charged current spectrum is $\approx 19 \%$. This possible background contribution is added to the prediction by weighting the $\nu_{\tau}$ events that are selected as $\nu_{\mu}$ charged current events for the probability $P\left(\nu_{\mu} \rightarrow \nu_{\tau}\right)$. In the same fashion, any subdominant oscillation of $\nu_{\mu}$ to $\nu_{e}$ are accounted for using the probability $P\left(\nu_{\mu} \rightarrow \nu_{e}\right)$. The efficiency for a charged current $\nu_{e}$ to be selected in the $\nu_{\mu}$ charged current spectrum is $\approx 3.6 \%$.

For the prediction of the neutral current energy spectrum, events are weighted according to the probability $1-P\left(\nu_{\mu} \rightarrow \nu_{s}\right)$, which represents the probability for a starting $\nu_{\mu}$ to be in any of the active flavours at detection. In an equivalent fashion, only neutrinos that have oscillated into sterile neutrinos disappear from the spectrum. Background to the neutral current selected spectrum are also $\nu_{e}$ charged current events (weighted for $P\left(\nu_{\mu} \rightarrow \nu_{e}\right)$ ) and $\nu_{\tau}$ charged current events (weighted for $P\left(\nu_{\mu} \rightarrow \nu_{\tau}\right)$ ) which are selected as neutral current events. The efficiency of selecting $\nu_{\tau}$ charged current interactions in the neutral current spectrum is $\approx 79 \%$, while the one for charged current $\nu_{e}$ is $96 \%$. The oscillations into $\nu_{e}$ are dependent on the value of $\theta_{13}$ chosen in the analysis $\left(\theta_{13}=0^{\circ}, 12^{\circ}\right)$. 
The small number of beam $\nu_{e}$ that pass the charged current or neutral current selection are also propagated and added to the predicted spectrum, but it is assumed they do not oscillate as matter effects for the MINOS baseline can be neglected.

The predicted Far Detector spectra are fitted to the data using the following likelihood function:

$$
\chi^{2}=2 \cdot \sum_{i=1}^{n}\left[e_{i}-o_{i}+o_{i} \ln \left(\frac{o_{i}}{e_{i}}\right)\right]+\sum_{j=1}^{N} \frac{\epsilon_{j}^{2}}{\sigma_{j}^{2}}
$$

The first part of the equation is equivalent to the likelihood used in equation (6.7), where $e_{i}$ and $o_{i}$ represent the expected and observed values at each bin $i$ of the charged current and neutral current energy spectra. The second part of the equation represents a penalty term due to the fit of systematic errors: $\epsilon_{j}$ is the shift from the nominal value of the $j$ th systematic source considered and $\sigma_{j}$ is the uncertainty associated with the systematic source. The choice of the $N$ systematic error sources which are used in the fit and their uncertainty values are described in section 8.7. The fitting algorithm has been studied in [151]. The neutral current and charged current selected spectra are fitted at the same time, separated also by run period. The binning scheme chosen consists of $1 \mathrm{GeV}$ bins between 0-20 GeV, and one single bin between 20-120 GeV. The different beam conditions (target position, target decay and helium in the decay pipe) are taken into account in the $\mathrm{MC}$ and the predictions are fitted to the corresponding data.

\subsection{Systematic errors on the oscillation parameters}

Numerous checks have been performed from the whole Neutral Current Working Group to make sure that data at the Near and Far detectors were in agreement with the expectation from MC. The stability of the selected events in space (within different region of the detectors) and time have been monitored. In addition to the plots shown in this thesis, a more comprehensive collection of data quality and stability plots is available in [152].

Also, a large set of possible systematic error sources have been considered and studied 
by the working group and the effects they have on the analysis is reported here. As the Near Detector input is used to predict the Far Detector spectrum, some systematic sources which affect both the detectors in the same way are highly reduced by the two detector approach. Section 8.7.1 presents the sources of uncertainties that are common to the two detectors. The absolute hadronic shower scale, even if common to both detectors, has a large impact on the analysis as it shifts the location of the oscillation minimum. The errors that involve only one of the two detectors have in general large effects on the analysis: they are treated in section 8.7.3.

\subsubsection{Sources of systematic errors common to the two detectors}

- Absolute hadronic calibration: the MINOS Calibration Detector was calibrated with stopping muons and the response to hadronic showers at a test beam was measured. The analysis has shown a bias of $5.7 \%$ in the measurement of the absolute hadronic energy scale [88]. The calibration was performed using the single particle energy resolution; any simulation uncertainty of the whole shower modelling also affects the energy scale and needs to be added in quadrature. The intranuclear rescattering, i.e. the rescattering of the hadrons produced in the neutrino interaction before they emerge from the nucleus, plays an important role in the shower energy uncertainty. The estimate of the error is done using the INTRANUKE simulation package $[94,153]$ and has been evaluated in [154] by varing the cross sections, branching ratios and formation length of the modelled shower. The overall uncertainty due to the MC shower modelling is to be added in quadrature with the $5.7 \%$ of the energy scale data measurements. The overall effect reaches $10 \%$ at lower shower energies and becomes approximatively asymptotical to $6.6 \%$ at higher shower energies.

- Track energy: For a muon that stops in the detector, its energy is measured from range. The calibration of the range-energy conversion has been studied at the test beam using the Calibration Detector and its uncertainty is equal to $2 \%$ [80]. This 
uncertainty is applied at the Near and Far detector on the selected charged current sample only. The energy of exiting muons is obtained by fitting the muon track to determine their curvature. Checks on the consistency of the energy measurement from range and from curvature have been performed. The $2 \%$ uncertainty is conservatively applied also on exiting tracks.

- Charged current background in the neutral current spectrum: The charged current background selected in the neutral current spectrum has an uncertainty of 15\%. The systematic error has been evaluated as explained in [30] by using different beam configurations to reduce the impact of the wrong modelling of the MC neutral current and charged current cross sections.

- Neutral current background in the charged current spectrum: The charged current selection has been shown to be very pure (see figure 8.23). The uncertainty associated with the neutral current background has been evaluated to be $25 \%$ of the background events in [155]. The method uses data and MC events in which the track hits have been removed to properly compare the efficiency of reconstructing small showers.

- Cross section uncertainty: Neutrino cross sections are simulated by the NEUGEN package [94]. At low energies (less than $1 \mathrm{GeV}$ ), quasi-elastic scattering and resonant production dominate. In the simulation package, the two processes are modelled using two axial mass parameters for the two types of interactions: the uncertainties found on the quasi-elastic and resonant axial masses are 15\% [57]. The KNO parameters in NEUGEN are scaling parameters that change the multiplicity distributions of the hadronization in the final state. From studies in [156] it has been found that the neutral current analysis is predominatly sensitive to a mismodelling in the charged current cross section.

- Beam uncertainties: The systematic uncertainties for the neutrino analysis have 
been evaluated in [137] as the overall contributions from the hadron production, beam optics and focusing, target position and decay uncertainties.

\subsubsection{Sources of systematic errors causing differences between the two detectors}

- The Near Detector preselection uncertainty: the uncertainty related to the preselection at the Near Detector was described in section 8.2.4 and caused an uncertainty in the Near Detector energy spectrum up to $10 \%$ for showers with energy less than $1 \mathrm{GeV}$. As the prediction of the Far Detector spectrum uses the Near Detector spectrum as an input, the same uncertanty is expected also on the Far Detector energy spectrum as a consequence.

- The Far Detector preselection uncertainties (cosmic and noise removal): the uncertainties related to the preselection cuts at the Far Detector have been described in section 8.4 and in [147]. In particular the error associated with the cosmic ray cuts affects the Far Detector energy spectrum as shown in table 8.1.

- Relative hadronic calibration: the conversion from detector response to hadronic energy is achieved by the calibration chain in both data and MC for both detectors, as explained in chapter 4 and in [100]. The Near Detector to Far Detector relative energy scale uncertainty has been evaluated to be $2.1 \%$.

- Relative Near and Far Detector Normalisation: the relative normalisation between the detectors is one of the most important uncertainties in both the charged current and neutral current energy spectra. It has been studied in [98]. The normalisation uncertainty arises from various sources: the uncertainty in the measurement of the integrated number of proton on targets at the Far Detector has been quantified as 1\%. The fiducial mass of the Far Detector has an uncertainty of less of $0.5 \%$ due to differences in the modelling of the steel and scintillator thickness in the 
Far Detector data and MC. There is also an uncertainty associated with imperfect geometrical modelling of the Near Detector, which has been quantified as $0.7 \%$. The biggest systematic component comes from the difference in the Near Data/MC and Far Data/MC reconstruction efficiencies. The evaluation of reconstuction efficiencies has been performed in [98] and has been quantified to be $2 \%$. The technique adopted uses selected charged current events in data and MC, which have the hits corresponding to the track removed. The "new" cluster of hits is processed by the reconstruction algorithm again. With this method, it is possible to determine the absolute efficiency of reconstructing neutral current events and compare it between data and MC. After summing in quadrature the components, the overall normalization systematic error has been found to be $2.2 \%$ constant over the whole energy spectrum.

Figure 8.24 shows the effect that the systematic errors have on the neutral current energy spectrum (left) and the charged current one (right) at the Near Detector (top), at the Far Detector without performing any extrapolation (centre) and at the Far Detector after performing the "Far/Near" extrapolation (bottom). While some of the systematic effects have a large impact on the energy spectrum of each of the two detectors separately, their effect is cancelled by the "Far/Near" extrapolation.

\subsubsection{Effects of the systematic uncertainties on the oscillation parameters}

To evaluate the effects of the various sources of systematic errors on the oscillation analysis, high statistic fake data (built from the MC with known oscillation parameters) have been simulated introducing the $\pm 1 \sigma$ uncertainty for each systematic error. The modified simulated data have been fitted to the nominal MC and the shifts on the oscillation parameters have been quantified. The simulated data were oscillated at $\Delta m^{2}=2.43 \times 10^{-3} \mathrm{eV}^{2}$, $\theta_{23} \approx 34.4^{\circ}, \theta_{24} \approx 6^{\circ}$ and $\theta_{34} \approx 11^{\circ}$. The values of the angle chosen allow to study the effects of the systematics far from the angle range boundaries. This allows not to artificially 

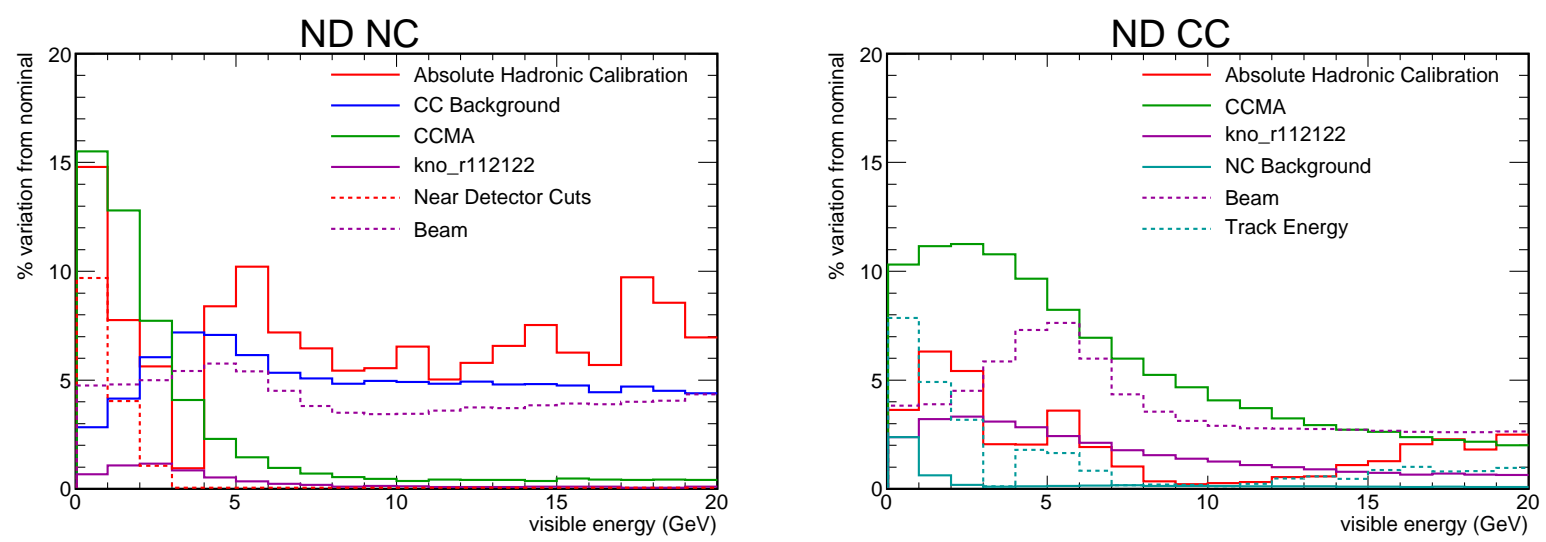

FD NC
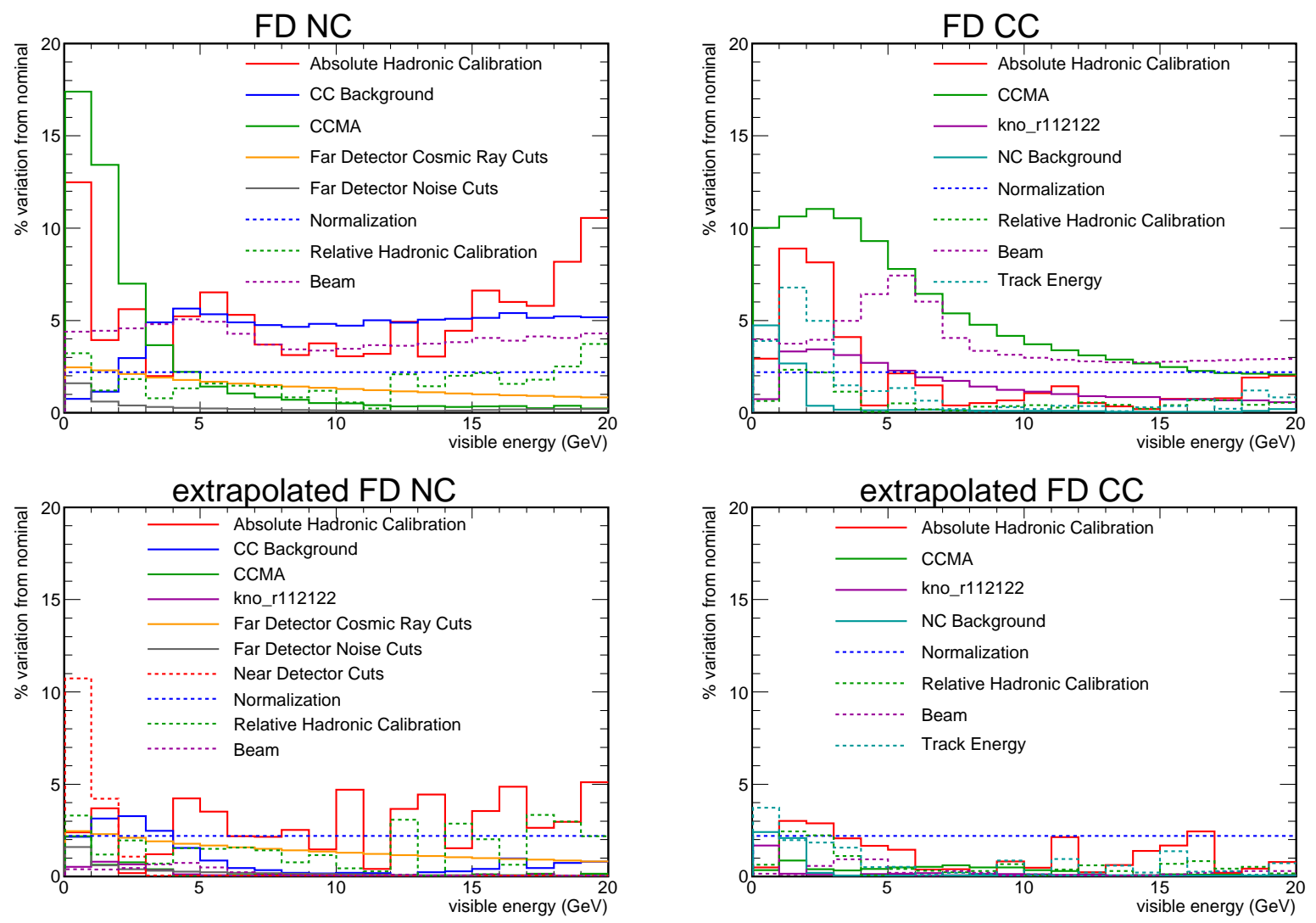

Figure 8.24: The effects that the systematic errors have on the neutral current selected spectrum (left) and charged current selected spectrum (right) at the Near Detector (top), at the Far Detector (centre) and at the Far Detector after the "Far/Near" extrapolation is performed (bottom). The effect of systematic errors common to both the detectors is largely reduced. 

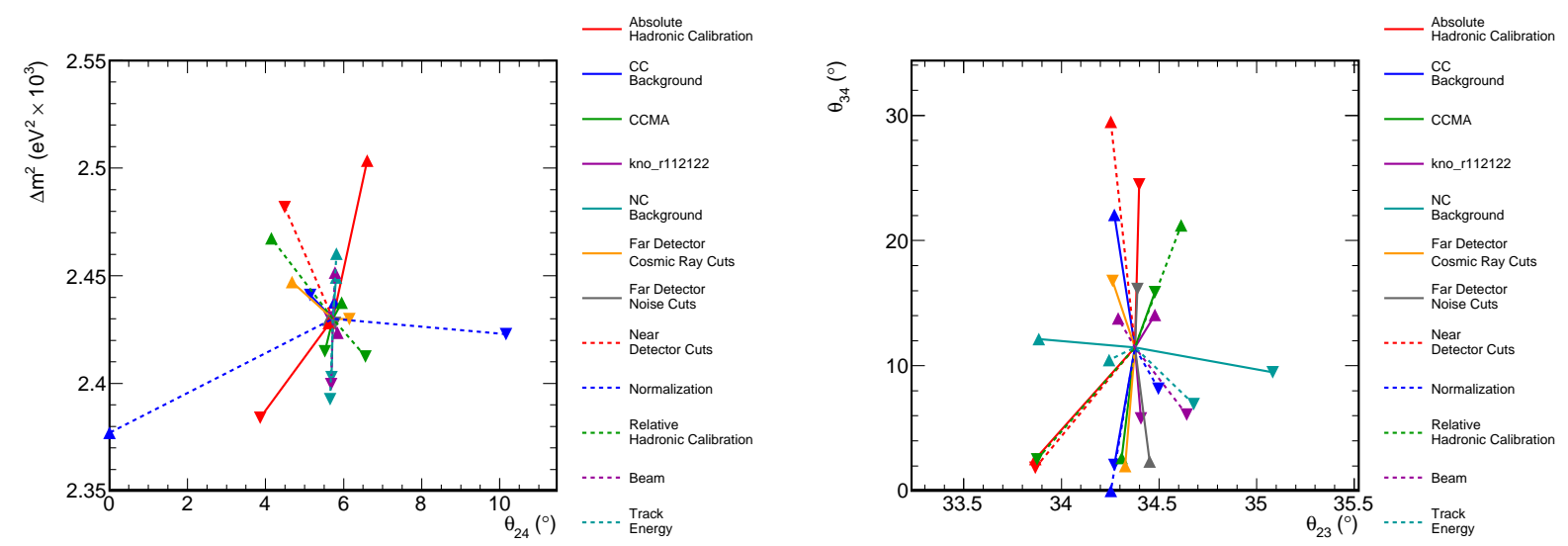

Figure 8.25: The shifts caused by each single systematic error on the best fit parameters. The up-pointing (down-pointing) triangle represents the effect of $+1 \sigma(-1 \sigma)$ of the systematic error studied. On the left plot the effects considered are on the parameters $\left(\theta_{24}, \Delta m^{2}\right)$, on the right plot for $\left(\theta_{23}, \theta_{34}\right)$.

underestimate the shifts in the parameters introduced by the systematic errors. Figure 8.25 shows the effects that altering the fake data by $\pm 1 \sigma$ has on the oscillation parameters. The major systematics, which will be taken into account in the final oscillation analysis, are: the absolute and relative hadronic shower energy, the relative normalisation, the Near Detector and Far Detector preselection and the charged current background in the neutral current sample. The six systematic uncertainties are used as penalty terms in the evaluation of the fit $\chi^{2}$ in equation (8.10).

\subsection{Sensitivity studies}

Preliminary sensitivity studies on the oscillation model have been performed using high statistic MC scaled to the data statistic of $7 \times 10^{20}$ protons on target. The fake data have been generated from the MC with known input parameters and they have been fitted to the nominal MC. The input values for the fake data have been chosen to be: $\Delta m^{2}=$ $2.43 \times 10^{-3} \mathrm{eV}^{2}, \theta_{23}=45^{\circ}, \theta_{24}=0^{\circ}$ and $\theta_{34}=0^{\circ}$. The fitted parameters are in excellent agreement with the input ones, showing no bias in the fit. Figure 8.26 shows the results for the $90 \%$ C.L. two-dimensional contours obtained from the sensitivity studies for the angle parameters $\left(\theta_{34}, \theta_{24}\right),\left(\theta_{23}, \theta_{24}\right)$ and $\left(\theta_{34}, \theta_{23}\right)$. In principle contours can also be made for 


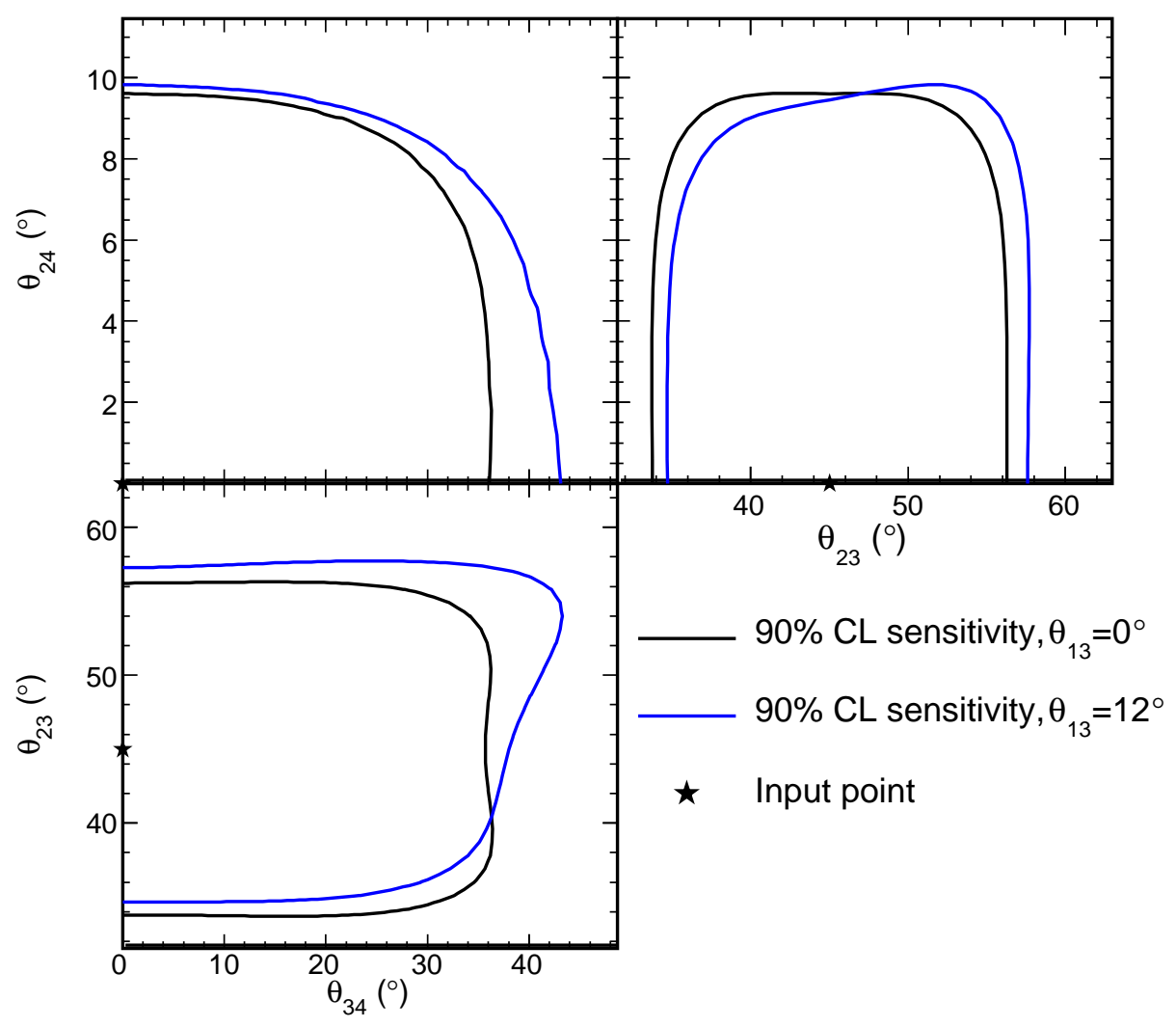

Figure 8.26: Statistical sensitivities for the angle parameters (90\% C.L.) for high statistic $\mathrm{MC}$ scaled to the real data statistics of $7 \times 10^{20}$ protons on target. No systematic uncertainty is applied at this stage. Black is in the case $\theta_{13}=0^{\circ}$, blue in the case $\theta_{13}=12^{\circ}$. The top left plot shows the countor for $\left(\theta_{34}, \theta_{24}\right)$, the top right plot is for $\left(\theta_{23}, \theta_{24}\right)$ and the bottom plot is for $\left(\theta_{34}, \theta_{23}\right)$.

$\Delta m^{2}$ but the best sensitivity for this parameter is obtained from a specific charged current analysis such as [57]. The contours are statistical only (the effect of the systematic errors has not been simulated). 

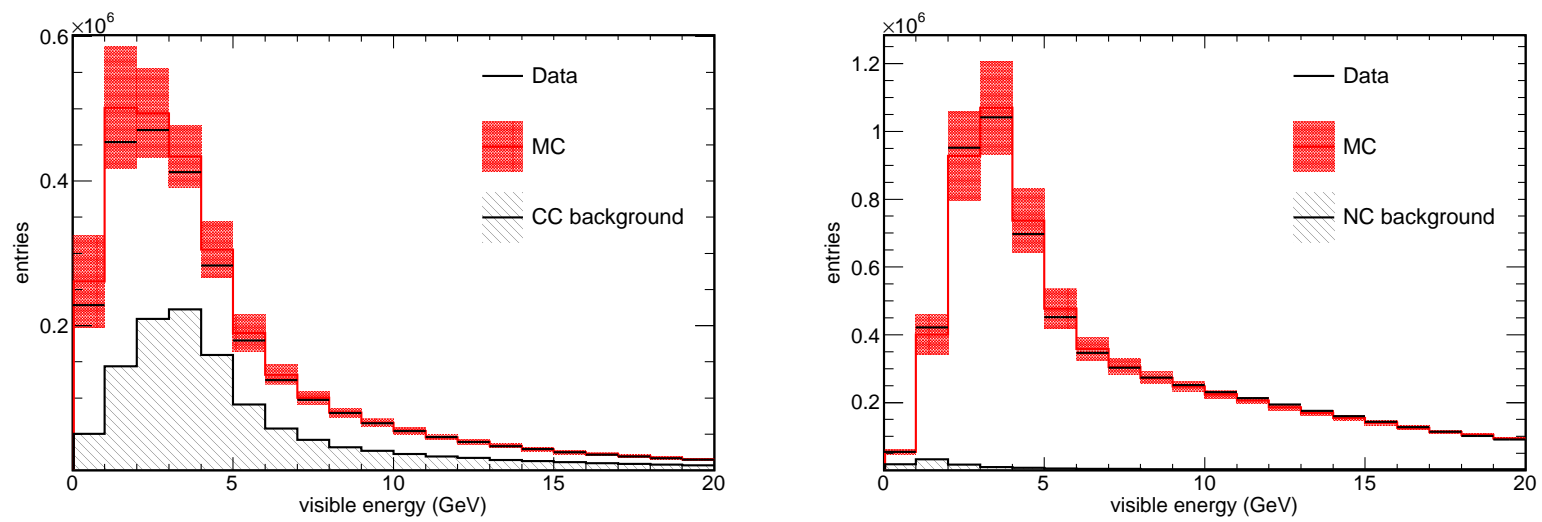

Figure 8.27: The plots show the neutral current selected spectrum (left) and the charged current one (right) at the Near Detector. Data are in black, MC is in red (the systematic error band is shown in hatched red) and the wrongly selected background is in hatched black.

\subsection{Results of the sterile neutrino oscillation analysis}

\subsubsection{Data versus three-flavour oscillation prediction}

Figure 8.27 shows the Near Detector energy spectra for neutral current events (left) and charged current events (right). Data are in black, MC is shown in red (the systematic error band is shown in hatched red) and the background is shown in the black hatched histogram.

The selection cuts and the extrapolation of the Far Detector MC prediction have been performed. Data have been analysed and checks versus the expectation for oscillation in the three-flavor model, where the oscillation probabilities are expressed in general in equation (2.19). The parameters used for the prediction are: $\Delta m_{12}^{2}=7.59 \times 10^{-5} \mathrm{eV}^{2}$, $\theta_{12}=34.4^{\circ}$ (the last two parameters come from a joint fit of SNO+KAMLAND [20]). The phase $\delta_{1}$ has been set to $=3 \pi / 2$. The specific oscillation parameters which MINOS can measure have been chosen to be $\Delta m^{2}=2.43 \times 10^{-3} \mathrm{eV}^{2}$ and $\sin ^{2}\left(2 \theta_{23}\right)=1$, as from [57]. $\theta_{13}$ has been kept fixed at two values: $0^{\circ}$ and $12^{\circ}$. The $\mathrm{MC}$ prediction has been corrected for the near detector data/MC ratio as explained in section 8.6 which describes the extrapolation technique. Figure 8.28 shows the selected neutral current (top) and charged 
current (bottom) spectra at the Far Detector. Data are in black, the MC prediction in the $\theta_{13}=0^{\circ}$ case is shown in red (with the associated systematic error) and the prediction using $\theta_{13}=12^{\circ}$ is in blue. The charged current $\nu_{\mu}$ background (common to both of the cases) is in hatched black and the charged current $\nu_{e}$ background if $\theta_{13}=12^{\circ}$ is shown in hatched blue.

The number of data events selected in the neutral current spectrum is $802 \pm 28.32$ (stat.) \pm 42.36 (syst.). The MC expectation for the three-flavour model is 754.22 events in the case of $\theta_{13}=0^{\circ}$ (13.32 events are expected as charged current $\nu_{\tau}$ interactions that are selected as neutral current). In the case $\theta_{13}=12^{\circ}, 59.31$ events are expected as $\nu_{e}$ events coming from the subdominant $\nu_{\mu}$ to $\nu_{e}$ oscillations in the neutral current spectrum, making the overall expected number of neutral current selected events to be 812.13 events (0.96 $\nu_{\tau}$ background events are lost due to the subdominant oscillations). The number of data events which are charged current selected is $1462 \pm 38.24$ (stat.) \pm 35.59 (syst.). The expected number of events selected as charged current in the three-flavour model is $1394.62(1395.87)$ for $\theta_{13}=0^{\circ}\left(\theta_{13}=12^{\circ}\right)$, with a background of $2.59(2.40) \nu_{\tau}$ events. The background events from the beam $\nu_{e}$ are 24.33 events for the neutral current selected spectrum and 0.18 events for the charged current selected spectrum.

\subsubsection{Data fitted to the $m_{4} \gg m_{3}$ model}

The fit to the sterile neutrino model in which $\left|\Delta m_{43}^{2}\right| \approx \mathcal{O}\left(1 \mathrm{eV}^{2}\right)$ has been performed. The best fit values for the parameters minimise the $\chi^{2}$ in equation (8.10), with the nuisance parameters for the systematics also being fitted. Table 8.2 shows the fitted parameters for the case of $\theta_{13}=0^{\circ}, 12^{\circ}$. Table 8.3 shows the values for the systematic shift parameters $\epsilon_{j}$ expressed as a fraction of the uncertainty $\sigma$ given for each of the six systematic source.

Figure 8.29 shows the Far Detector energy spectra for neutral current selected events including the results of the fit. Data are in black, the best fit MC for $\theta_{13}=0^{\circ}$ is presented in red and the best fit $\mathrm{MC}$ for $\theta_{13}=12^{\circ}$ is in blue. The wrongly selected charged current 

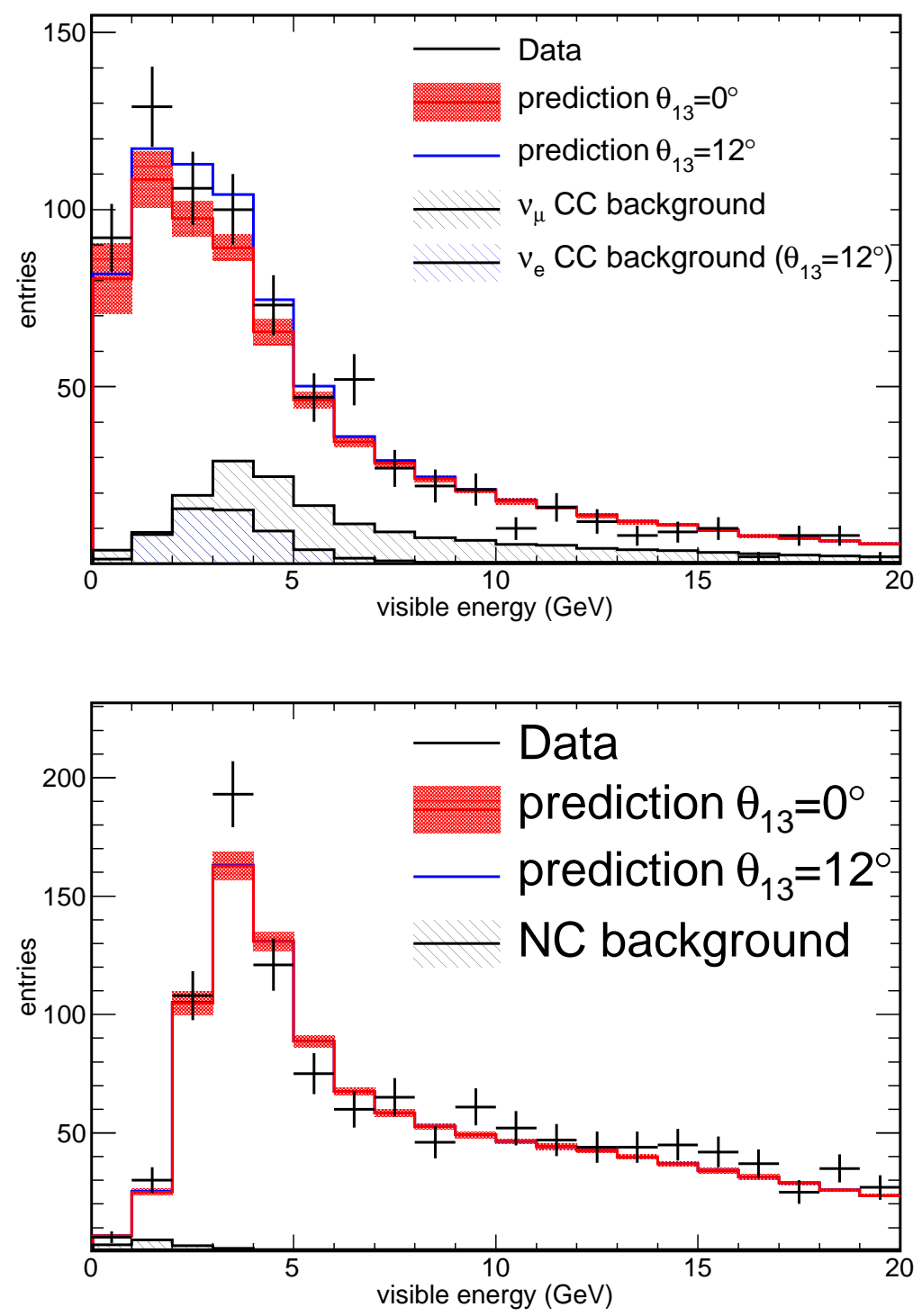

Figure 8.28: The plots show the neutral current selected (top) and charged current selected spectra (bottom) at the Far Detector. Data are in black; the MC prediction in case of a three-flavour oscillation $\left(\theta_{13}=0^{\circ}\right.$ indicates no $\nu_{e}$ appearance) is superimposed in red with the systematic error band. The full three-flavour MC expectation with $\nu_{e}$ appearance $\left(\theta_{13}=12^{\circ}\right)$ is indicated in blue. The black hatched histogram is the background due to $\nu_{\mu}$ charged current interactions and the hatched blue histogram is the background due to $\nu_{e}$ appearance at $\theta_{13}=12^{\circ}$ (negligible for the charged current selected sample). The oscillation parameters chosen are consistent with [57]. 


\begin{tabular}{|c||c|c|c|c|c|}
\hline$\theta_{13}\left(^{\circ}\right)$ & $\Delta m^{2}\left(\mathrm{eV}^{2} \times 10^{3}\right)$ & $\theta_{23}\left(^{\circ}\right)$ & $\theta_{24}\left(^{\circ}\right)$ & $\theta_{34}\left(^{\circ}\right)$ & $\chi^{2} / \mathrm{DOF}$ \\
\hline 0 & $2.43_{-0.18}^{+0.20}$ & $39.09_{-4.89}^{+16.71}$ & $0.00_{-0.00}^{+4.79}$ & $0.00_{-0.00}^{+17.39}$ & $129.181 / 122$ \\
\hline 12 & $2.43_{-0.18}^{+0.21}$ & $40.27_{-5.17}^{+14.64}$ & $0.00_{-0.00}^{+5.99}$ & $0.00_{-0.00}^{+24.57}$ & $127.310 / 122$ \\
\hline
\end{tabular}

Table 8.2: The table shows the results to the fit of the sterile neutrino mixing model to the Far Detector data for two values of $\theta_{13}=0^{\circ}, 12^{\circ}$. The error on the parameters represent the $68 \%$ C.L.

\begin{tabular}{|c||c|c|c|c|c|c|}
\hline$\theta_{13}\left(^{\circ}\right)$ & Abs.Hadr.Cal. & Rel.Hadr.Cal. & Normalisation & CC bkg & ND presel. & FD presel. \\
\hline 0 & $0.00 \sigma$ & $-0.34 \sigma$ & $0.69 \sigma$ & $-0.80 \sigma$ & $-0.62 \sigma$ & $0.42 \sigma$ \\
\hline 12 & $0.13 \sigma$ & $-0.31 \sigma$ & $0.44 \sigma$ & $-0.26 \sigma$ & $-0.62 \sigma$ & $0.00 \sigma$ \\
\hline
\end{tabular}

Table 8.3: The table shows the results for the fit of the nuisance parameters $\epsilon_{j}$ in equation (8.10) for the fit in the case $\theta_{13}=0^{\circ}$ and $\theta_{13}=12^{\circ}$. The results are given as a function of $\sigma$, which represents the estimate of each systematic error.

$\nu_{\mu}$ are shown as a background for the neutral current spectrum. As the best fit values for $\theta_{24}$ and $\theta_{34}$ is $0^{\circ}$, the best fit spectrum is consistent to a three-flavour prediction for $\left|\Delta m_{31}^{2}\right|=2.43 \times 10^{-3} \mathrm{eV}^{2}$. If $\theta_{13} \neq 0^{\circ}$, the survival probability $P\left(\nu_{\mu} \rightarrow \nu_{\mu}\right)$ loses its symmetry around $\theta_{23}=\pi / 4$ (as will be shown in figure 8.31): the data seems to favour $\theta_{23}$ slightly smaller than $\pi / 4$ although the $\Delta \chi^{2}$ values are not enough to draw any conclusion.

The errors for the fitted parameters in table 8.2 are given at the $68 \%$ C.L. $\left(\Delta \chi^{2}=1\right)$. The $90 \%$ C.L. intervals are given by the $\Delta \chi^{2}=2.71$. The $\Delta \chi^{2}$ projection as a function of $\Delta m^{2}$ is shown in figure 8.30. Figure 8.31 shows the $90 \%$ C.L. for the angle parameters. From the $\Delta \chi^{2}$ projections, that is when minimising the $\chi^{2}$ against all other parameters, $\theta_{24}<7^{\circ}\left(8^{\circ}\right)$ and $\theta_{34}<24^{\circ}\left(38^{\circ}\right)$ at $90 \%$ C.L. if $\theta_{13}=0^{\circ}\left(12^{\circ}\right)$. The bigger dependence in the value of $\theta_{34}$ from $\theta_{13}$ is consistent with the fact that $\theta_{34}$ is uniquely determined by the fit to the neutral current selected spectrum, which is affected by $\nu_{e}$ appearance. Instead, the angle $\theta_{24}$ is mainly determined by the fit to the charged current spectrum and the dependence on the value of $\theta_{13}$ is weak. Figure 8.32 shows the $90 \%$ C.L. two-dimentional contours for the angle parameters, which are obtained from $\Delta \chi^{2}=4.61$. 


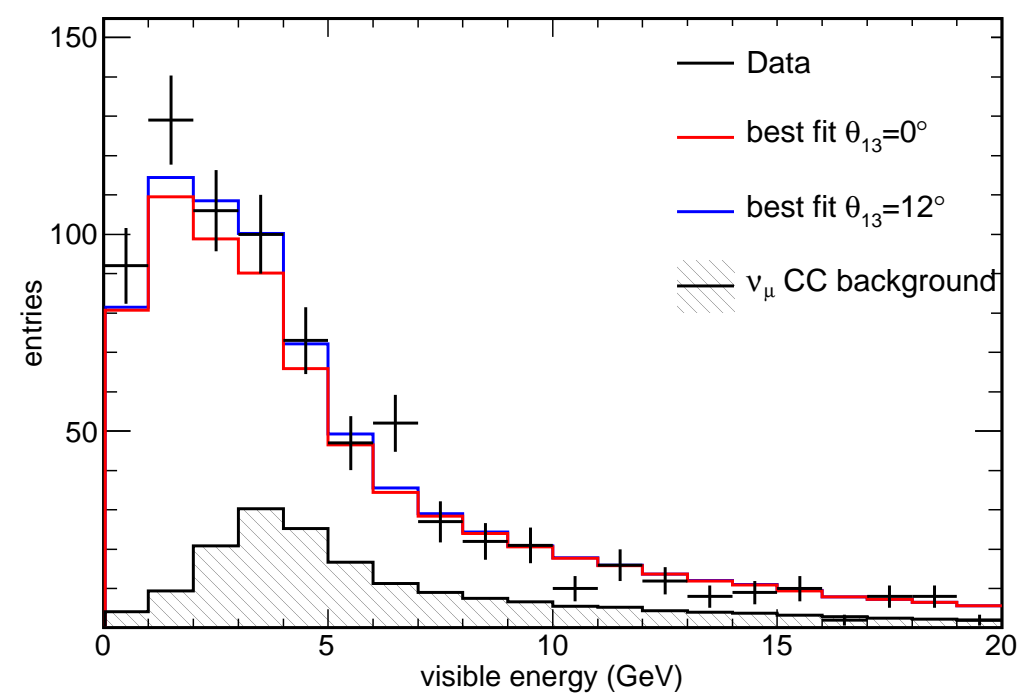

Figure 8.29: The plot shows the neutral current selected spectrum at the Far Detector. Data are in black (the error bars represent the statistical uncertainty), the best fit MC for $\theta_{13}=0^{\circ}$ is in red, the best fit $\mathrm{MC}$ for $\theta_{13}=12^{\circ}$ is in blue. The background due to charged current $\nu_{\mu}$ is in hatched black.

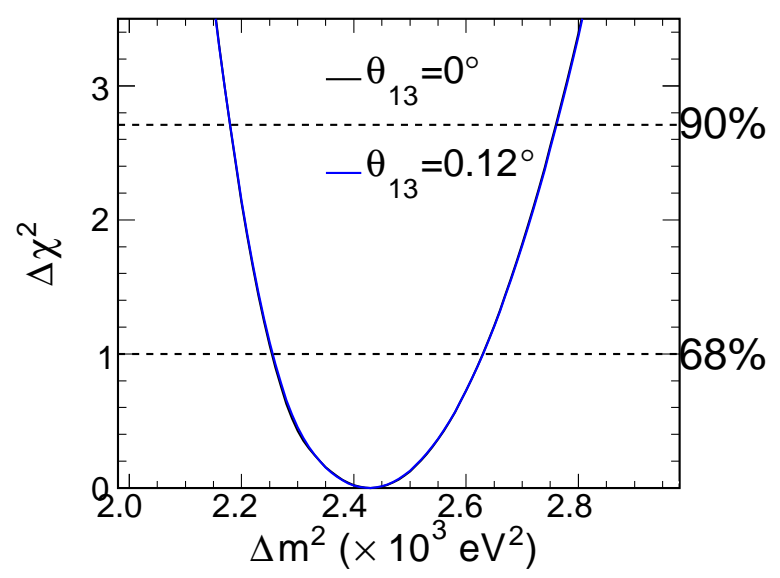

Figure 8.30: The figure shows the $\Delta \chi^{2}$ projection for the $\Delta m^{2}$ parameter. As expected there is no difference between the case $\theta_{13}=0^{\circ}$ (black) and $\theta_{13}=12^{\circ}$ (blue) as the mass splitting parameter is mainly determined by the charged current spectrum, which is very weakly dependent on $\theta_{13}$. 


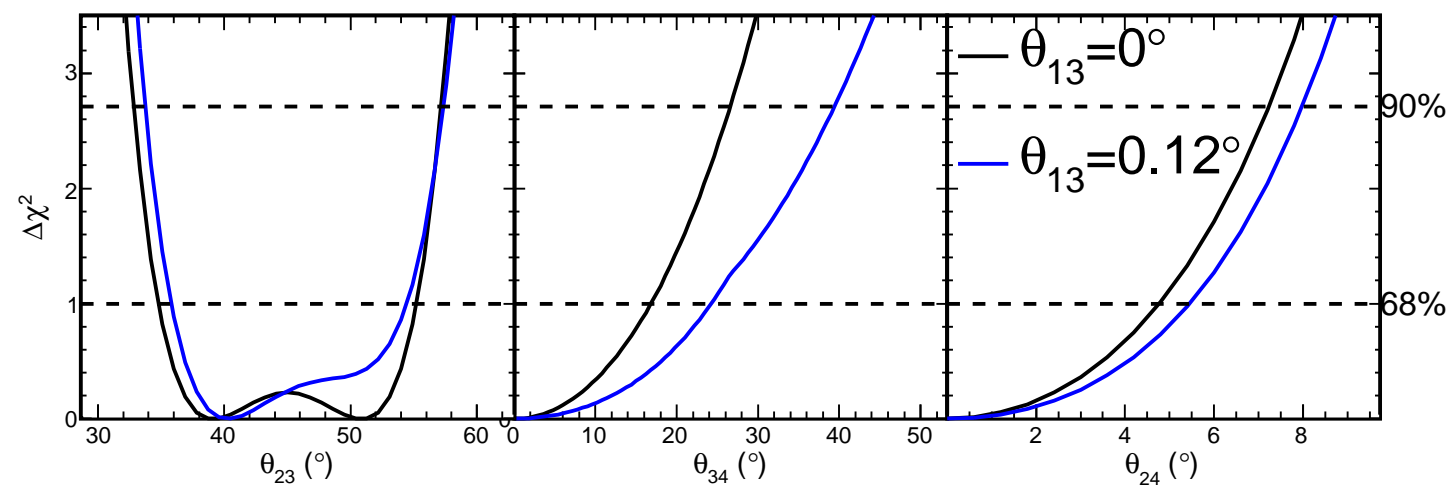

Figure 8.31: The figure shows the $\Delta \chi^{2}$ projections for the three angle parameters.

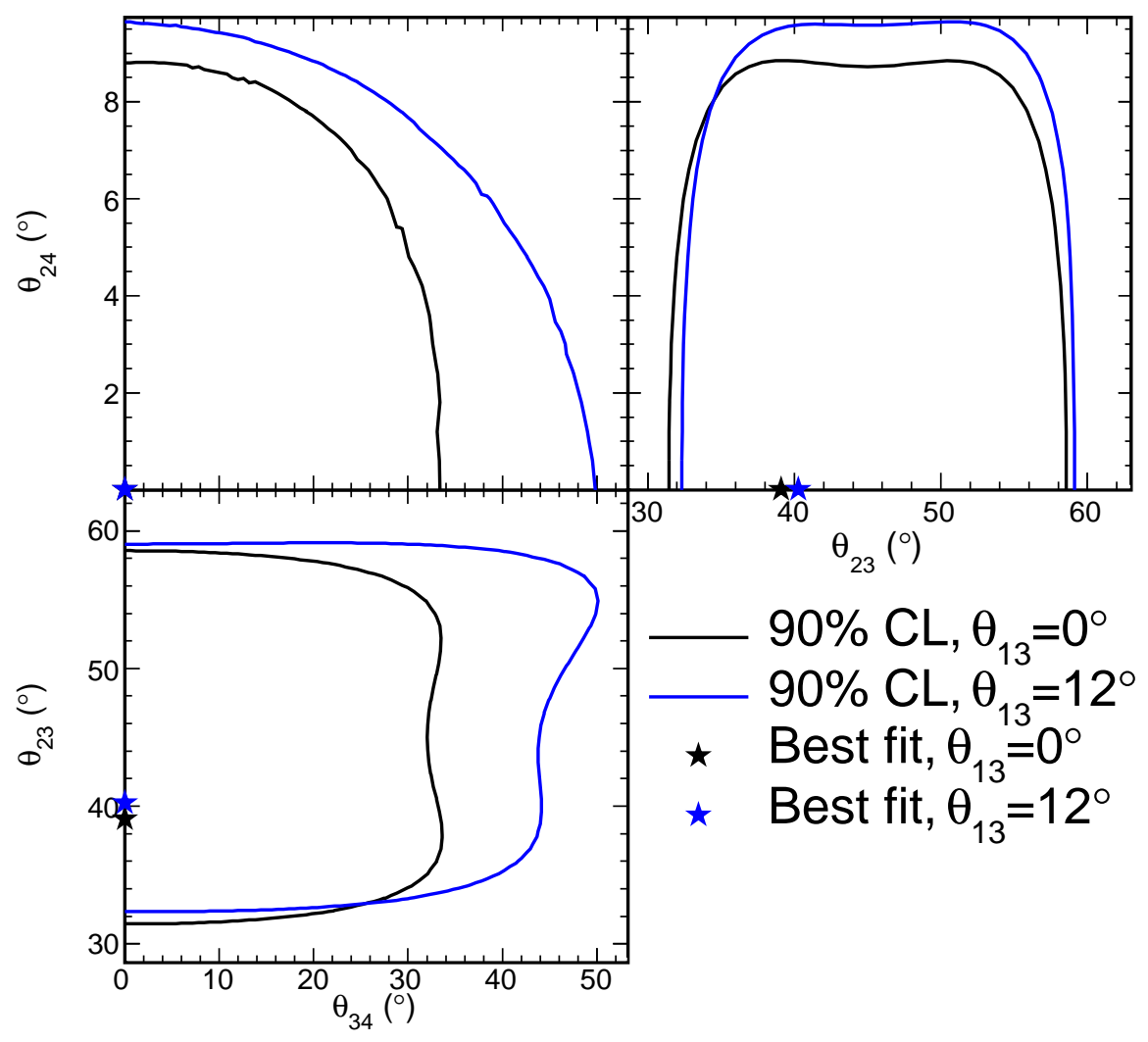

Figure 8.32: The plots show the $90 \%$ C.L. contours for the angle parameters as obtained from the fit using $\theta_{13}=0^{\circ}$ (black) and $\theta_{13}=12^{\circ}$ (blue). 


\subsection{Conclusions}

An analysis of neutrino oscillations allowing a sterile neutrino mixing has been performed in the model $\left|\Delta m_{43}^{2}\right| \approx \mathcal{O}\left(1 \mathrm{eV}^{2}\right)$. This mass splitting is consistent with the anomalous mass splitting observed by the LSND experiment. Although another experiment, MiniBooNE, collected neutrino data which seem to discard the LSND results, the angle parameters measured by MINOS are an input for phenomenologists to develop models to accommodate results from both experiments, given the different running conditions (antineutrinos versus neutrinos). The analysis presented in this thesis is an improvement compared to the limits on the mixing angles obtained in the previous official MINOS neutral current analysis [30], which are shown in figure 3.2. The improvements are mainly due to the increase in statistics (the dataset analysed is nearly double than the previous one). MINOS has released official preliminary results on the updated sterile neutrino analysis [58]. Thanks to a different charged current selection [141], the results on the mixing angles are more stringent: $\theta_{23}=$ $45_{-7.2}^{\circ+7.2}, \theta_{24}=0.00^{\circ+4.8}$ and $\theta_{34}=0.00^{\circ+16.8}$ under the assumption that $\theta_{13}=0^{\circ}$.

The further data taking in MINOS is unclear at the moment: there are no specific plans to further develop the analysis proposed in this thesis. Nevertheless, given the new antineutrino results from MiniBooNE [74], an antineutrino neutral current analysis in MINOS, with the same theoretical model as presented in this theses, could be very interesting. At the present moment the statistics of the neutral current antineutrino spectrum is very poor due to the small neutral current cross section. A MINOS neutral current analysis with improved statistics (running with neutrinos) may be interesting also for the dependence of the Far Detector neutral current spectrum on appearance of products of neutrino oscillations as a background.

The background in the neutral current spectrum coming from the truly charged current $\nu_{\tau}$ interactions accounts for too few events $\left(13\right.$ expected events for the $7 \times 10^{20}$ protons on target analysis) for allowing any discrimination about $\nu_{\tau}$ appearance in MINOS. The small statistics of appearing $\nu_{\tau}$ is due to the fact that the oscillation probability $P\left(\nu_{\mu} \rightarrow \nu_{\tau}\right)$ has 
its maximum at $1.4 \mathrm{GeV}$ at the MINOS baseline, while the threshold for $\nu_{\tau}$ charged current interactions is $3.5 \mathrm{GeV}$ due to the mass of the $\tau$ lepton. $\nu_{\tau}$ charged current interactions are a background to the neutral current selected spectrum as the branching ratio for $\tau$ to decay hadronically is about $60 \%$ [12] and the branching ratio for $\tau$ decay into an electron which is then identified as a neutral current event is about 18\% [12]. In addition, the $\tau$ decay has an escaping $\nu_{\tau}$ as final product of the interaction: as a result the decay products that are detected are smeared in energy, making their identification even more difficult.

On the other hand, $\nu_{e}$ appearance may be detected more easily. In the analysis performed in this thesis, it has been decided to keep the value of $\theta_{13}$ and $\delta$ fixed. In future analysis, if the statistics of protons on target allows, $\theta_{13}$ could be fitted in the same way as the other angle parameters. Using the data collected in this thesis, a fit to the three-flavour oscillation model with $\theta_{13}$ fitted has been performed, minimising the $\chi^{2}$ in equation (8.10) (including systematics). As $\delta$ influences the $P\left(\nu_{\mu} \rightarrow \nu_{e}\right)$ probability only mildly (see figure 2.14), its value has been kept fixed to $3 \pi / 2$ for simplicity. The new fit to the data finds as best fit value for $\theta_{13}=10.71^{\circ}$. Figure 8.33 shows the $\Delta \chi^{2}$ projection as a function of $\theta_{13}$. The value of $\theta_{13}=0^{\circ}$ can be discarded only at a level of $1.4 \sigma$, given by the square root of the difference between the $\chi^{2}$ value at $\theta_{13}=0^{\circ}$ and the $\chi^{2}$ at the minimum. As $\theta_{13}$ has a boundary at zero, the use of Gaussian distributed errors is not properly correct, but it gives a good indication of the potentiality of the analysis. The $90 \%$ upper limit is $\theta_{13} \approx 17^{\circ}$. Using the plot from the official MINOS $\nu_{e}$ appearence analysis in figure 2.14 and considering the value returned by the fit for $\theta_{23}$ and the fixed value for the phase $\delta$, the value of $\theta_{13}$ that corresponds to the $90 \%$ C.L is $11.6^{\circ}$ in the case of inverted hierarchy (worst case). Selecting a purest $\nu_{e}$ sample (as it happens in [63]) increases the sensitivity on $\theta_{13}$.

In addition to more statistics, a reduction of certain systematic errors needs to be performed to gain sensitivity. In particular, the systematic errors which are not common to the two detectors need to be reduced. The preselection cuts at the Near Detector and Far Detector at the moment are treated as completely independent of one another. In principle 


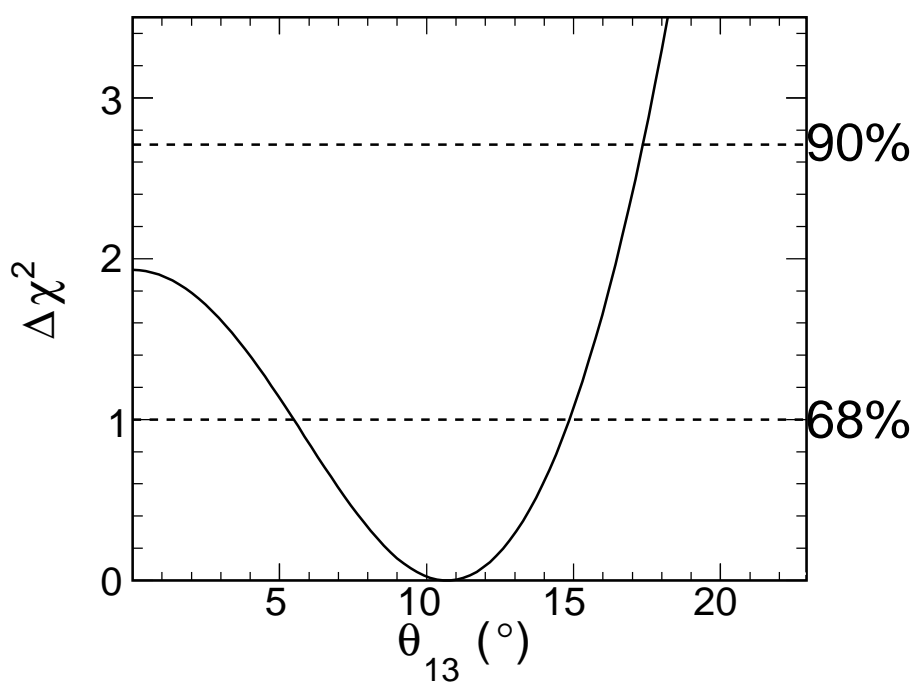

Figure 8.33: The figure shows the $\Delta \chi^{2}$ projection as a function of $\theta_{13}$.

this is true only in first approximation. The cuts are meant to deal with the different event rate and backgrounds at the two detectors. Still, some cuts (a minimum consecutive plane cut for example) will have the same effect at both detectors as the selection variable have and the systematic error could be reduced by considering that there is a partial correlation between the two preselection techniques and part of the effect cancels out in the extrapolation stage. 


\section{Chapter 9}

\section{Conclusion}

The MINOS experiment, which is in the final stages of operation, has immensely contributed to the neutrino oscillation measurements setting the world best limit on the atmospheric mass splitting $\left|\Delta m_{\text {atm }}^{2}\right|$, providing antineutrino oscillation parameters in the atmospheric sector and confirming the $\mathrm{CHOOZ}$ limit on $\theta_{13}$.

This thesis has addressed another beam oscillation analysis: neutrino oscillation measurements allowing mixing to a sterile neutrino with additional mass splitting $\mathcal{O}\left(1 \mathrm{eV}^{2}\right)$. Even though the limits on sterile neutrinos measured in this thesis are not stringent, mainly due to the limited statistics of the neutral current sample, the analysis provides important checks for the neutrino physics community. The analysis uses a combined fit to the charged current and neutral current spectra to obtain oscillation measurements. Systematic errors on the neutral current spectrum play an important role in the sterile measurement analysis. This is due to a combination of high impurity of the neutral current sample, smearing of the hadronic shower energy due to the missing neutrino energy and reconstruction failures which affect low energy events.

This thesis has covered both the analysis procedure and the study of some of the major systematic errors which affect the neutral current analysis. The drift calibration procedure

presented here has contributed to reduce the relative shower energy calibration uncertainty between the two detectors by removing the effect of the ageing on the detector response. 
Beam related uncertainties due to the production of hadrons off the carbon target have been addressed by comparing pion and kaon production ratios to the experimental data in the NA49 experiment. The kaon data in NA49 have been analysed (with other NA49 collaborators) for the first time in this thesis. Neutrino reconstruction failures, which modify the neutral current selected spectrum at the Near Detector due to the high neutrino flux, have been analysed and removed by cuts on specific variables. The rejection of the poorly reconstructed events has improved the shower energy resolution, the agreement between data and $\mathrm{MC}$ in the Near Detector and the purity of the Near Detector neutral current spectrum has been made comparable to the purity of the Far Detector neutral current spectrum.

In the remaining time of operation (at least one year or possibly more), the MINOS experiment will provide improved results on the charged current oscillation analysis, possibly increase the statistics of the anti-neutrino oscillation analysis (the anti-neutrino running needs to be coordinated with MINER $\nu \mathrm{A}$, a new neutrino cross section experiment sharing the NuMI beam with MINOS) and improve the limit on $\theta_{13}$ with more statistics and by performing a fit on the selected neutrino spectrum instead of using only the total number of events. MINOS is still statistically limited and has much to give to neutrino physics!

Now that both the solar and atmospheric oscillation mass splittings are well known, the main interest in the neutrino field is towards measuring $\theta_{13}$ or putting a stringent limit on it. Reactor experiments (Double-Chooz, Reno, Daya Bay) can access $\theta_{13}$ and new baseline experiments (T2K and $\mathrm{NO} \nu \mathrm{A}$ ) are also potentially sensitive to the $C P$ phase $\delta$. At the same time, the LSND anomaly is now a question more open than ever and future measurements of $\bar{\nu}_{\mu} \rightarrow \bar{\nu}_{e}$ are expected. 


\section{Bibliography}

[1] Precision electroweak measurements on the $\mathrm{Z}$ resonance, Physics Reports 427, 257 (2006).

[2] W. Pauli, Letter, addressed to participants of the Tübingen conference on radioactivity (1930), Available from the CERN Document server: http://cdsweb.cern.ch/ record/83282P.

[3] E. Fermi, Versuch einer Theorie der $\beta$-Strahlen. I., Zeitschrift für Physik 88, 161 (1934).

[4] F. Reines and C. Cowan, Detection of the Free Neutrino, Phys. Rev. 92, 830 (1953).

[5] F. Reines and C. Cowan, The Neutrino, Nature 178, 446 (1956).

[6] M. Goldhaber, L. Grodzins, and A. W. Sunyar, Helicity of Neutrinos, Phys. Rev. 109, 1015 (1958).

[7] G. Danby et al., Observation of High-Energy Neutrino Reactions and the Existence of Two Kinds of Neutrinos, Phys. Rev. Lett. 9, 36 (1962).

[8] Gargamelle Neutrino Collaboration: F. J. Hasert et al., Search for elastic muon neutrino electron scattering, Phys. Lett. B46, 121 (1973).

[9] Gargamelle Neutrino Collaboration: F. J. Hasert et al., Observation of neutrino-like interactions without muon or electron in the Gargamelle neutrino experiment, Phys. Lett. B46, 138 (1973). 
[10] Aachen-Brussels-CERN-Ecole Poly-Milan-Orsay-London Collaboration: J. Blietschau et al., Search for Strangeness Changing Neutral Currents Induced by Neutrinos in Gargamelle, Phys. Lett. B71, 231 (1977).

[11] S. L. Glashow, J. Iliopoulos, and L. Maiani, Weak Interactions with Lepton-Hadron Symmetry, Phys. Rev. D 2, 1285 (1970).

[12] C. Amsler et al., Review of Particle Physics, Phys. Lett. B667, 1 (2008).

[13] DONUT Collaboration: K. Kodama et al., Observation of Tau Neutrino Interactions, Phys. Lett. B504, 218 (2001).

[14] R. Ardito et al., CUORE: A cryogenic underground observatory for rare events, (2005).

[15] R. Neilson et al., Characterization of large area APDs for the EXO-200 detector, Nuclear Instruments and Methods in Physics Research A 608, 68 (2009).

[16] SuperNEMO Collaboration: R. Arnold et al., Probing New Physics Models of Neutrinoless Double Beta Decay with SuperNEMO, (2010).

[17] T. J. Loredo and D. Q. Lamb, Bayesian analysis of neutrinos observed from supernova SN 1987A, Phys. Rev. D 65, 063002 (2002).

[18] Z. Maki, M. Nakagawa, and S. Sakata, Remarks on the unified model of elementary particles, Prog. Theor. Phys. 28, 870 (1962).

[19] B. Pontecorvo, Inverse beta processes and nonconservation of lepton charge, Sov. Phys. JETP 7, 172 (1958).

[20] M. C. Gonzalez-Garcia, M. Maltoni, and J. Salvado, Updated global fit to three neutrino mixing: status of the hints of $\theta_{13}>0,(2010)$.

[21] R. P. Litchfield, Neutrino induced events in the MINOS detectors, DPhil thesis, University of Oxford (2008). 
[22] J. J. Evans, Measuring neutrino oscillations with the MINOS experiment, DPhil thesis, University of Oxford (2008).

[23] S. J. Parke, CP violation in the Neutrino Sector (2008).

[24] L. Wolfenstein, Neutrino oscillations in matter, Phys. Rev. D17, 2369 (1978).

[25] S. P. Mikheev and A. Y. Smirnov, Resonance enhancement of oscillations in matter and solar neutrino spectroscopy, Sov. J. Nucl. Phys. 42, 913 (1985).

[26] T. Tabarelli de Fatis, Prospects of measuring $\sin ^{2}\left(2 \theta_{13}\right)$ and the sign of $\Delta m^{2}$ with a massive magnetized detector for atmospheric neutrinos, Eur. Phys. J. C24, 43 (2002).

[27] A. Donini, M. B. Gavela, P. Hernandez, and S. Rigolin, Neutrino mixing and CPviolation, Nucl. Phys. B 574, 23 (2000).

[28] A. Donini, M. Maltoni, D. Meloni, P. Migliozzi, and F. Terranova, 3+1 sterile neutrinos at the CNGS, J. High En. Phys. 12, 013 (2007).

[29] CHOOZ Collaboration: M. Apollonio et al., Search for neutrino oscillations on a long base-line at the CHOOZ nuclear power station, Eur. Phys. J. C27, 331 (2003).

[30] The MINOS Collaboration: P. Adamson et al., Search for sterile neutrino mixing in the MINOS long- baseline experiment, Phys. Rev. D81, 052004 (2010).

[31] M. Maltoni and T. Schwetz, Sterile neutrino oscillations after first MiniBooNE results, Phys. Rev. D76, 093005 (2007).

[32] R. Davis, D. S. Harmer, and K. C. Hoffman, Search for neutrinos from the sun, Phys. Rev. Lett. 20, 1205 (1968).

[33] GALLEX Collaboration: P. Anselmann et al., Solar neutrinos observed by GALLEX at Gran Sasso., Phys. Lett. B285, 376 (1992). 
[34] GALLEX Collaboration: W. Hampel et al., GALLEX Solar Neutrino Observations: Results for GALLEX IV, Phys. Lett. B447, 127 (1999).

[35] A. I. Abazov et al., Search for neutrinos from the Sun using the reaction ${ }^{71} \mathrm{Ga}\left(\nu_{e}, e^{-}\right){ }^{71} \mathrm{Ge}$, Phys. Rev. Lett. 67, 3332 (1991).

[36] SAGE Collaboration: J. Abdurashitov et al., Measurement of the Solar Neutrino Capture Rate by the Russian-American Gallium Solar Neutrino Experiment During One Half of the 22-Year Cycle of Solar Activity, ZhETP 122, 1 (2002).

[37] GNO Collaboration: M. Altmann et al., Complete results for five years of GNO solar neutrino observations, Phys. Lett. B616, 174 (2005).

[38] Kamiokande-II Collaboration: K. S. Hirata et al., Observation of ${ }^{8} \mathrm{~B}$ solar neutrinos in the Kamiokande-II detector, Phys. Rev. Lett. 63, 16 (1989).

[39] Super-Kamiokande Collaboration: J. Hosaka et al., Solar neutrino measurements in Super-Kamiokande-I, Phys. Rev. D73, 112001 (2006).

[40] SNO Collaboration: B. Aharmim et al., Electron Energy Spectra, Fluxes, and DayNight Asymmetries of ${ }^{8}$ B Solar Neutrinos from the 391-Day Salt Phase SNO Data Set, Phys. Rev. C72, 055502 (2005).

[41] KamLAND Collaboration: S. Abe et al., Precision Measurement of Neutrino Oscillation Parameters with KamLAND, Phys. Rev. Lett. 100, 221803 (2008).

[42] Borexino Collaboration: C. Arpesella et al., New Results on Solar Neutrino Fluxes from 192 Days of Borexino Data, Phys. Rev. Lett. 101, 091302 (2008).

[43] C. Kraus, SNO with liquid scintillator: SNO+, Progress in Particle and Nuclear Physics 57, 150 (2006), International Workshop of Nuclear Physics 27th course Neutrinos in Cosmology, in Astro, Particle and Nuclear Physics. 
[44] IMB Collaboration: J. M. LoSecco et al., Test of Neutrino Oscillations Using Atmospheric Neutrinos, Phys. Rev. Lett. 54, 2299 (1985).

[45] Kamiokande Collaboration: K. Hirata et al., Observation of a small atmospheric $\nu_{\mu} / \nu_{e}$ ratio in Kamiokande, Phys. Lett. 280, 146 (1992).

[46] NUSEX Collaboration: M. Aglietta et al., Experimental Study of Atmospheric Neutrino Flux in the NUSEX Experiment, Europhys. Lett. 8, 611 (1989).

[47] Fréjus Collaboration: K. Daum et al., Determination of the atmospheric neutrino spectra with the Fréjus detector, Z. Phys. C66, 417 (1995).

[48] Soudan2 Collaboration: W. Allison et al., Measurement of the atmospheric neutrino flavour composition in Soudan 2, Phys. Lett. B391, 491 (1997).

[49] Super-Kamiokande Collaboration: Y. Fukuda et al., Measurement of a small atmospheric $\nu_{\mu} / \nu_{e}$ ratio, Phys. Lett. B433, 9 (1998).

[50] W. A. Mann, Atmospheric neutrinos and the oscillations bonanza, Int. J. Mod. Phys. A15S1, 229 (2000).

[51] Super-Kamiokande Collaboration: Y. Fukuda et al., Evidence for oscillation of atmospheric neutrinos, Phys. Rev. Lett. 81, 1562 (1998).

[52] Super-Kamiokande Collaboration: Y. Ashie et al., A measurement of atmospheric neutrino oscillation parameters by Super-Kamiokande I, Phys. Rev. D71, 112005 (2005).

[53] Super-Kamiokande Collaboration: C. Yanagisawa, Recent results from SuperKamiokande, in Frontiers in Contemporary Physics 2001, Vanderbilt University, Nashville, Tennessee, USA (2001).

[54] Super-Kamiokande Collaboration: Y. Ashie et al., Evidence for an oscillatory signature in atmospheric neutrino oscillations, Phys. Rev. Lett. 93, 101801 (2004). 
[55] Super-Kamiokande Collaboration: K. Abe et al., A measurement of atmospheric neutrino flux consistent with tau neutrino appearance, Phys. Rev. Lett. 97, 171801 (2006).

[56] K2K Collaboration: M. H. Ahn et al., Measurement of neutrino oscillation by the K2K experiment, Phys. Rev. D74, 072003 (2006).

[57] MINOS Collaboration: P. Adamson et al., Measurement of Neutrino Oscillations with the MINOS Detectors in the NuMI Beam, Phys. Rev. Lett. 101, 131802 (2008).

[58] P. Vahle, New results from MINOS - NEUTRINO2010 (2010).

[59] OPERA Collaboration: N. Agafonova et al., Observation of a first $\nu_{\tau}$ candidate in the OPERA experiment in the CNGS beam, Phys. Lett. B691, 138 (2010).

[60] NOvA Collaboration: D. S. Ayres et al., NOvA proposal to build a 30-kiloton off-axis detector to study neutrino oscillations in the Fermilab NuMI beamline, (2004).

[61] Y. Itow et al., The JHF-Kamioka neutrino project, (2001), hep-ex/0106019.

[62] MINOS Collaboration: P. Adamson et al., Search for muon-neutrino to electronneutrino transitions in MINOS, Phys. Rev. Lett. 103, 261802 (2009).

[63] The MINOS Collaboration: P. Adamson et al., New constraints on muon-neutrino to electron-neutrino transitions in MINOS, (2010).

[64] R. Patterson, New results for $\nu_{\mu} \rightarrow \nu_{e}$ oscillations in MINOS - Fermilab Joint Experimental-Theoretical Seminar (2010).

[65] Double Chooz Collaboration: F. Ardellier et al., Double Chooz: A search for the neutrino mixing angle $\theta_{13}$, hep-ex/0606025 (2006).

[66] Daya Bay Collaboration: X. Guo et al., A precision measurement of the neutrino mixing angle $\theta_{13}$ using reactor antineutrinos at Daya Bay, hep-ex/0701029 (2007). 
[67] RENO Collaboration: J. K. Ahn et al., RENO: An Experiment for Neutrino Oscillation Parameter $\theta_{13}$ Using Reactor Neutrinos at Yonggwang, (2010).

[68] H. Nunokawa, S. J. Parke, and J. W. F. Valle, CP Violation and Neutrino Oscillations, Prog. Part. Nucl. Phys. 60, 338 (2008).

[69] LSND Collaboration: C. Athanassopoulos et al., Evidence for anti- $\nu_{\mu} \rightarrow$ anti- $\nu_{e}$ oscillations from the LSND experiment at the Los Alamos Meson Physics Facility, Phys. Rev. Lett. 77, 3082 (1996).

[70] LSND Collaboration: C. Athanassopoulos et al., Evidence for $\nu_{\mu} \rightarrow \nu_{e}$ oscillations from LSND, Phys. Rev. Lett. 81, 1774 (1998).

[71] KARMEN Collaboration: B. Armbruster et al., Upper limits for neutrino oscillations muon-antineutrino to electron-antineutrino from muon decay at rest., Phys. Rev. D65, 112001 (2002).

[72] The MiniBooNE Collaboration: A. A. Aguilar-Arevalo et al., A Search for electron neutrino appearance at the $\Delta m^{2} \sim 1 \mathrm{eV}^{2}$ scale, Phys. Rev. Lett. 98, 231801 (2007).

[73] The MiniBooNE Collaboration: A. A. Aguilar-Arevalo et al., A Search for Electron Antineutrino Appearance at the $\Delta m^{2} \sim 1 \mathrm{eV}^{2}$ scale, Phys. Rev. Lett. 103, 111801 (2009).

[74] R. V. de Water, Updated anti-neutrino oscillation results from MiniBooNE - NEUTRINO2010 (2010).

[75] Super-Kamiokande Collaboration: S. Fukuda et al., Tau neutrinos favored over sterile neutrinos in atmospheric muon neutrino oscillations, Phys. Rev. Lett. 85, 3999 (2000).

[76] Super-Kamiokande Collaboration: A. Habig, Discriminating between $\nu_{\mu} \rightarrow \nu_{\tau}$ and $\nu_{\mu} \rightarrow \nu_{\text {sterile }}$ in atmospheric $\nu_{\mu}$ oscillations with the Super-Kamiokande detector, (2001). 
[77] Super-Kamiokande Collaboration: M. Shiozawa, Experimental results on atmospheric neutrinos in Super-Kamiokande-I, (2002), Contributed to Neutrino 2002, Munich, Germany, 25-30 May 2002.

[78] MINOS Collaboration: P. Adamson et al., Search for active neutrino disappearance using neutral- current interactions in the MINOS long-baseline experiment, Phys. Rev. Lett. 101, 221804 (2008).

[79] S. E. Kopp, The NuMI Beam at FNAL and its use for Cross Section Measurements, (2007).

[80] MINOS Collaboration: P. Adamson et al., Study of Muon Neutrino Disappearance using the Fermilab Main Injector Neutrino Beam, Phys. Rev. D77, 072002 (2008).

[81] MINOS Collaboration: D. G. Michael et al., The Magnetized Steel and Scintillator Calorimeters of the MINOS Experiment, Nucl. Instrum. Meth. A569, 190 (2008).

[82] J. Hartnell, NuMI Muon Antineutrinos in MINOS - Fermilab Joint ExperimentalTheoretical Seminar (2009).

[83] MINOS Collaboration: P. Adamson et al., First observations of separated atmospheric $\nu_{\mu}$ and $\bar{\nu}_{\mu}$ events in the MINOS detector, Phys. Rev. D73, 072002 (2006).

[84] MINOS Collaboration: P. Adamson et al., Measurement of the atmospheric muon charge ratio at TeV energies with MINOS, Phys. Rev. D76, 052003 (2007).

[85] MINOS Collaboration: P. Adamson et al., Charge-separated atmospheric neutrinoinduced muons in the MINOS far detector, Phys. Rev. D75, 092003 (2007).

[86] P. Adamson et al., Neutrino and antineutrino inclusive charged-current cross section measurements with the MINOS near detector, Phys. Rev. D 81, 072002 (2010).

[87] S. Kopp et al., Measurement of the NuMI Neutrino Flux, MINOS-doc-5977 (2010). 
[88] P. Adamson et al., Calibration of the MINOS Near and Far Detector Readout Systems, To be submitted to Nucl. Instrum. Meth..

[89] MINOS Collaboration: A. Cabrera et al., Comparisons of the MINOS Near and Far Detector Readout Systems at a Test Beam, Nucl. Instrum. Meth. A609, 106 (2009).

[90] M. A. Kordosky, Hadronic Interactions in the MINOS Detectors, PhD thesis University of Texas at Austin (2004).

[91] A. Fasso, A. Ferrari, P. R. Sala, and J. Ranft, FLUKA: Status and prospects for hadronic applications, Contributed to the International Conference on Advanced Monte Carlo for Radiation Physics, Particle Transport Simulation and Applications (MC 2000), Lisbon, Portugal, 23-26 October (2000).

[92] M. Campanella, A. Ferrari, P. R. Sala, and S. Vanini, First Calorimeter Simulation with the FLUGG Prototype, Technical Report ATL-SOFT-99-004 CERN Geneva (1999).

[93] A. I. Himmel, The NuMI beam simulation with Flugg, MINOS-doc-6316 (2010).

[94] H. Gallagher, The NEUGEN neutrino event generator, Nucl. Phys. Proc. Suppl. 112, 188 (2002).

[95] GEANT-Detector description and simulation tool, CERN Program Library, Long Writeup, W5013, http://wwwasd.web.cern.ch/wwwasd/geant/index.html.

[96] C. Zeitnitz and T. A. Gabriel, The GEANT - CALOR interface and benchmark calculations of ZEUS test calorimeters, Nucl. Instrum. Meth. A349, 106 (1994).

[97] R. E. Kalman, Trans. ASME, J. Bas. Eng. 82D, 35 (1960).

[98] P. A. Rodrigues, A sterile neutrino search for the MINOS experiment, DPhil thesis, University of Oxford (2010). 
[99] T. Yang, A study of muon neutrino to electron neutrino oscillations in the MINOS experiment, PhD thesis Stanford University (2009).

[100] The MINOS Calibration Group, 2009 Position Paper on Calibration of Runs I-II-III, MINOS-doc-3941 (2009).

[101] N. Tagg et al., Performance of Hamamatsu 64-anode photomultipliers for use with wavelength-shifting optical fibres, Nucl. Instrum. Meth. A539, 668 (2005).

[102] K. Lang et al., Characterization of 1600 Hamamatsu 16-anode photomultipliers for the MINOS Far detector, Nucl. Instrum. Meth. A545, 852 (2005).

[103] J. Hartnell, Measurement of the Calorimetric Energy Scale in MINOS, DPhil thesis, University of Oxford (2005).

[104] N. Tagg, Gain Calibration Systematics, MINOS-doc-4865 (2008).

[105] R. Pittam, Electronics Stability, MINOS-doc-1580 (2006).

[106] C. Andreopoulos, P. Stamoulis, and G. Tzanakos, Temperature Effects on M16 PMTs, NuMI-Note-SCINT-988 (2002).

[107] P. Miyagawa, A. Weber, and C. Perry, Measurements of Gain and Noise Properties of Hamamatsu M64 PMT's, NuMI-L-590 (2000).

[108] T. K. Gaisser, Cosmic rays and particle physics (Cambridge Univ. Press, Cambridge, 1990).

[109] MINOS Collaboration: P. Adamson et al., Observation of muon intensity variations by season with the MINOS far detector, (2009).

[110] J. D. Jong, Measurement of the underground atmospheric muon charge ratio between 225 mwe and 2100 mwe using the MINOS detectors, MINOS-doc-8080 (2009).

[111] N. Tagg, Drift Calibrations: Notes on recent discoveries, MINOS-doc-1203 (2005). 
[112] J. Mitchell, Comparison of ND Data Quality Selections, MINOS-doc-6530 (2009).

[113] D. A. Petyt, FD Data Quality Position Paper for summer 2007 CC analysis, MINOSdoc-3152 (2007).

[114] L. Landau, On the energy loss of fast particles by ionization, J Phys USSR 8, 201 (1944).

[115] J. W. Harris and B. Muller, The search for the quark-gluon plasma, Ann. Rev. Nucl. Part. Sci. 46, 71 (1996).

[116] NA49 Collaboration: S. Afanasev et al., The NA49 large acceptance hadron detector, Nucl. Instrum. Meth. A430, 210 (1999).

[117] O. Chvala, Transverse Phenomena in Pion Production in Hadronic Collisions at SPS energy, PhD thesis Charles University in Prague (2006).

[118] D. Barna, Strange particle production in proton-proton collisions at $158 \mathrm{GeV}, \mathrm{PhD}$ thesis Eotvos Lorand University (2002).

[119] NA49 Collaboration: C. Alt et al., Inclusive production of charged pions in $\mathrm{p}+\mathrm{C}$ collisions at 158-GeV/c beam momentum, Eur. Phys. J. C49, 897 (2007).

[120] M. V. Makariev, Inclusive production of charged pions in proton-proton and protoncarbon collisions at $158 \mathrm{GeV} / \mathrm{c}$ beam momentum, $\mathrm{PhD}$ thesis University of Sofia (2006).

[121] NA49 Collaboration: T. Anticic et al., Inclusive production of protons, anti-protons and neutrons in $\mathrm{p}+\mathrm{p}$ collisions at $158 \mathrm{GeV} / \mathrm{c}$ beam momentum, Eur. Phys. J. C65, 9 (2010).

[122] D. Varga, Cross section determination using full and Empty target data subtraction, NA49-DOC (2003). 
[123] G. I. Veres, Baryon Momentum Transfer in Hadronic and Nuclear Collisions at the CERN NA49 Experiment, PhD thesis Eotvos Lorand University (2001).

[124] R. P. Feynman, Very High-Energy Collisions of Hadrons, Phys. Rev. Lett. 23, 1415 (1969).

[125] D. Varga, Study of the truncated mean ionization distribution, NA49-DOC (2007).

[126] C. A. X. Pattison, Measurement of Charged Hadron Yields for Atmospheric Neutrino Studies, DPhil thesis, University of Oxford (2005).

[127] NA49 Collaboration: C. Alt et al., Inclusive production of charged pions in p p collisions at 158-GeV/c beam momentum, Eur. Phys. J. C45, 343 (2006).

[128] D. Varga, Study of Inclusive and Correlated Particle Production in Elementary Hadronic Interactions, PhD thesis University of Budapest (2003).

[129] The NA49 Collaboration: T. Anticic et al., Inclusive production of charged kaons in $\mathrm{p}+\mathrm{p}$ collisions at $158 \mathrm{GeV} / \mathrm{c}$ beam momentum and a new evaluation of the energy dependence of kaon production up to collider energies, (2010).

[130] M. Kreps, Reinteraction in the hydrogen target of the NA49 experiment, NA49-DOC (2003).

[131] T. Sjostrand et al., High-energy-physics event generator with PYTHIA 6.1, Comp. Phys. Comm. 135, 238 (2001).

[132] M. Bonesini, A. Marchionni, F. Pietropaolo, and T. Tabarelli de Fatis, On particle production for high energy neutrino beams, Eur. Phys. J. C20, 13 (2001).

[133] D. S. Barton et al., Experimental study of the $A$ dependence of inclusive hadron fragmentation, Phys. Rev. D 27, 2580 (1983).

[134] S. M. Seun, Measurement of the $\pi / K$ Ratio from the NuMI Target, PhD thesis Harvard University (2007). 
[135] A. V. Lebedev, Ratio of Pion Kaon Production in proton Carbon Interactions, PhD thesis Harvard University (2007).

[136] M. Gazdzicki, Z. Fodor, and G. Vesztergombi, Study of Hadron Production in HadronNucleus and Nucleus-Nucleus Collisions at the CERN SPS, Technical Report SPSCP-330. CERN-SPSC-2006-034 CERN Geneva (2006), revised version submitted on 2006-11-06 12:38:20.

[137] M. Dorman, Beam Fit Position Paper, MINOS-doc-7146 (2010).

[138] Z. Pavlovich, Observation of disappearance of muon neutrinos in the NuMI beam, $\mathrm{PhD}$ thesis University of Texas at Austin (2008).

[139] R. E. Armstrong, Muon neutrino disappearance at MINOS, PhD thesis Indiana University (2009).

[140] R. E. Armstrong, A comparison of the $\pi^{+} / \pi^{-}$ratio with NA49 as a function of $p_{T}$, MINOS-doc-5798 (2009).

[141] R. Ospanov, A Measurement of Muon Neutrino Disappearance with the MINOS Detectors and NuMI Beam, PhD thesis University of Texas at Austin (2008).

[142] T. M. Raufer, A study of neutrino oscillations in MINOS, DPhil thesis, University of Oxford (2007).

[143] A. Sousa, A Multivariate Discriminant method applied to Selection of Neutral Current Events in MINOS, MINOS-doc-3274 (2007).

[144] R. Pittam, A search for sterile neutrino at the MINOS experiment, DPhil thesis, University of Oxford (2010).

[145] C. Backhouse, Estimating shower energies using a kNN-based method - Position Paper, MINOS-doc-6868 (2010). 
[146] J. Coelho and G. Pawloski, Incorporating intensity profile in Monte Carlo, MINOSdoc-6032 (2009).

[147] J. D. Jong, Position paper for the RunI-III NC FD cleaning, MINOS-doc-7165 (2010).

[148] S. Avvakumov et al., Spontaneous light emission from fibers in MINOS, Nuclear Instruments and Methods in Physics Research Section A: Accelerators, Spectrometers, Detectors and Associated Equipment 545, 145 (2005).

[149] P. A. Rodrigues, Do we need a CC selection in the NC analysis?, MINOS-doc-6638 (2009).

[150] B. List, Why and When to Optimize Efficiency Times Purity, ETH Zurich internal note (2002).

[151] C. Backhouse, Systematics Interpolation Update, MINOS-doc-5701 (2009).

[152] P. A. Rodrigues, NC 7e20 data quality plots, MINOS-doc-7129 (2010).

[153] R. Merenyi et al., Determination of pion intranuclear rescattering rates in $\nu_{\mu}-N e$ versus $\nu_{\mu}-D$ interactions for the atmospheric $\nu$ flux, Phys. Rev. D 45, 743 (1992).

[154] M. Kordosky, H. Gallagher, and S. Dytman, Shower Energy Scale Uncertainty for the Run I+II CC Analysis, MINOS-doc-4309 (2008).

[155] J. S. Marshall, A Study of Muon Neutrino Disappearance with the MINOS Detectors and the NuMI Neutrino Beam, DPhil thesis, University of Cambridge (2008).

[156] A. Sousa and R. Pittam, NC Extrapolation Systematics for $f_{\text {sterile }}$ Measurement Comparison, MINOS-doc-3565 (2007). 